\title{
The Hydrophosphination of Bicyclo[1.1.0]butane- 1-carbonitriles
}

\author{
John A. Milligan ${ }^{\dagger}$, Carl A. Busacca, ${ }^{\ddagger *}$ Chris H. Senanayake, ${ }^{\dagger *}$ and Peter Wipf ${ }^{\dagger *}$ \\ ${ }^{\dagger}$ Department of Chemistry, University of Pittsburgh, Pittsburgh, Pennsylvania 15260, United States \\ ${ }^{\ddagger}$ Chemical Development, Boehringer-Ingelheim Pharmaceuticals, Inc., 900 Ridgebury Road, Ridgefield, \\ Connecticut 06877, United States
}

*E-mail: carl.busacca@boehringer-ingelheim.com (C.A.B.)

${ }^{*}$ E-mail: chris.senanayake@boehringer-ingelheim.com (C.H.S.)

*E-mail: pwipf@pitt.edu (P.W.)

\section{Table of Contents}

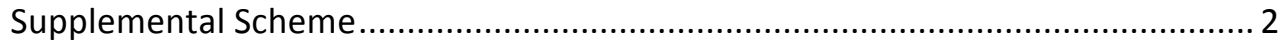

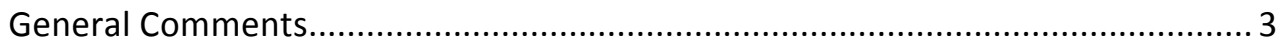

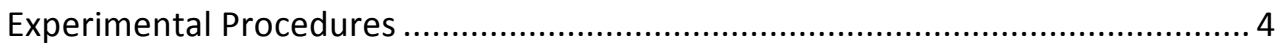

Differential Scanning Calorimetry Plot for Bicyclobutane 1 .................................. 24

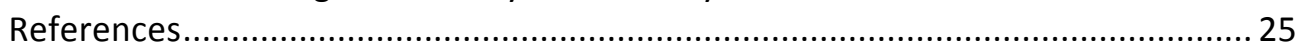

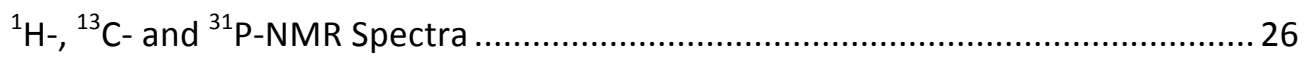

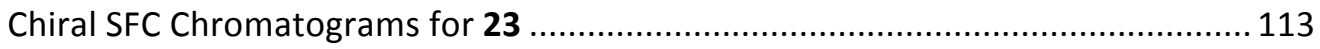


Supplemental Scheme 1: Hydrophosphination screening of strained carbocycles<smiles>Cc1cc(C)cc(NC(=O)C2CC2(C)C)c1</smiles><smiles>CC12CC1(C=O)C2</smiles><smiles>CC12CC1(C(N)=O)C2</smiles>

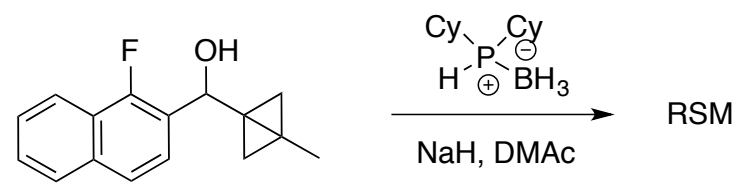<smiles>CCCCCC1(c2ccccc2)CC1C(O)c1ccc2ccccc2c1F</smiles><smiles>C=C1CC1(c1ccccc1)c1ccccc1</smiles><smiles>Cc1ccc(C2(c3ccccc3)CC2)cc1</smiles>
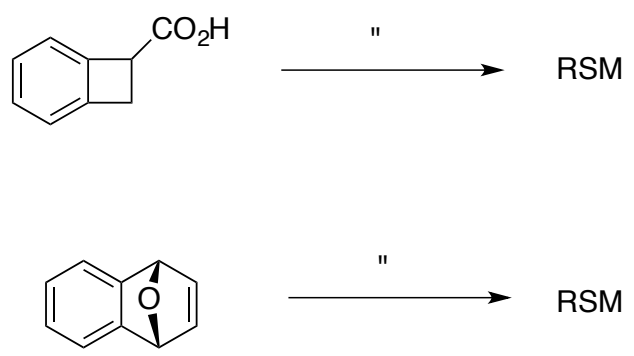


\section{General Comments}

All non-aqueous reactions were carried out under a nitrogen atmosphere in oven-dried glassware. Anhydrous tetrahydrofuran and diethyl ether were distilled from sodium benzophenone ketyl, and anhydrous dichloromethane and toluene were distilled from $\mathrm{CaH}_{2}$. All other solvents and reagents were used as obtained from commercial sources without further purification unless noted. Reactions were monitored via TLC using $250 \mu \mathrm{m}$ pre-coated silica gel 60 F254 plates, which were visualized with $254 \mathrm{~nm}$ and/or $365 \mathrm{~nm}$ UV light and by staining with $\mathrm{KMnO}_{4}\left(1.5 \mathrm{~g} \mathrm{KMnO}_{4}, 10 \mathrm{~g} \mathrm{~K}_{2} \mathrm{CO}_{3}\right.$, and $1.25 \mathrm{~mL} \mathrm{10 \%} \mathrm{NaOH}$ in $200 \mathrm{~mL}$ water). Chromatography was performed with Silicycle silica gel 60 (230-400 mesh) or using a CombiFlash automated

chromatography system with pre-packed $\mathrm{SiO}_{2}$ cartridges. ${ }^{1} \mathrm{H},{ }^{31} \mathrm{P}$ and ${ }^{13} \mathrm{C}$ NMR spectra were recorded on 300,400 , or $500 \mathrm{MHz}$ spectrometers, using the residual solvent as an internal standard. IR spectra were obtained on an ATR IR. HRMS data were obtained on a Single Quad Orbitrap mass spectrometer. 


\section{Experimental Procedures}

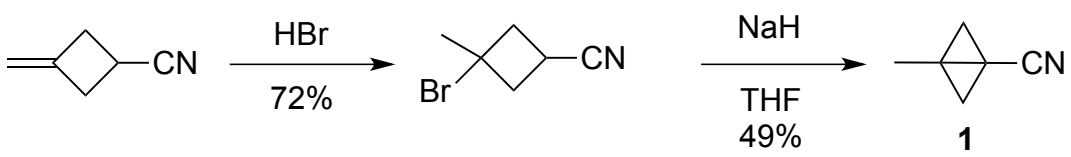

3-Methylbicyclo[1.1.0]butane-1-carbonitrile 1. $^{1}$

A mixture of 3-methylenecyclobutanecarbonitrile $(10.0 \mathrm{~g}, 0.107 \mathrm{~mol})$ and $48 \% \mathrm{HBr}(21 \mathrm{~mL}, 0.376$ mol, 3.5 eq) was vigorously stirred at room temperature for $24 \mathrm{~h}$. The reaction mixture was partitioned between water and ether $(100 \mathrm{~mL})$, the phases were separated, and the aqueous layer was extracted with ether $(2 \times 75 \mathrm{~mL})$. The combined ethereal solution was washed with water $(100 \mathrm{~mL})$ then sat. aq. $\mathrm{NaHCO}_{3}(100 \mathrm{~mL})$. The organic layer was dried $\left(\mathrm{MgSO}_{4}\right)$, filtered, and concentrated to afford the bromocyclobutane $(13.44 \mathrm{~g}, 77.20 \mathrm{mmol}, 72 \%)$ as a colorless liquid with a $d r$ 1.7:1. Characteristic signal for the major isomer: ${ }^{1} \mathrm{H}$ NMR $\left(300 \mathrm{MHz}, \mathrm{CDCl}_{3}\right) \delta$ $1.95(\mathrm{~s}, 3 \mathrm{H})$. Characteristic signal for the minor isomer: ${ }^{1} \mathrm{H} \mathrm{NMR}\left(300 \mathrm{MHz}, \mathrm{CDCl}_{3}\right) \delta 1.89(\mathrm{~s}, 3 \mathrm{H})$. A solution of bromide $(13.43 \mathrm{~g}, 77.17 \mathrm{mmol})$ in THF $(70 \mathrm{~mL})$ in a dry 3-necked $250-\mathrm{mL}$ round bottom flask fitted with a stopper, septum, and $\mathrm{N}_{2}$ inlet was treated with $\mathrm{NaH}(3.06 \mathrm{~g}, 77.2$ $\mathrm{mmol}$ ) in portions over $5 \mathrm{~min}$. The flask was stirred at $35^{\circ} \mathrm{C}$ for $12 \mathrm{~h}$, then at reflux for $5 \mathrm{~h}$. After cooling, the grey/brown reaction mixture was quenched with sat. aq. $\mathrm{NH}_{4} \mathrm{Cl}(25 \mathrm{~mL})$. The reaction mixture was then partitioned between diethyl ether $(100 \mathrm{~mL})$ and water $(100 \mathrm{~mL})$, the layers were separated, and the aqueous layer was re-extracted with ether $(75 \mathrm{~mL})$. The combined organic layers were washed with water $(100 \mathrm{~mL})$ and brine $(100 \mathrm{~mL})$, dried $\left(\mathrm{Na}_{2} \mathrm{SO}_{4}\right)$ and concentrated to afford a yellow-tinted cloudy liquid. The crude material was purified by bulb-to-bulb distillation (bath temp $75{ }^{\circ} \mathrm{C}, 2 \mathrm{mmHg}$ )) to afford bicyclobutane 1 (3.54 g, 38.0 mmol, 49\%) as a colorless liquid: ${ }^{1} \mathrm{H}$ NMR $\left(300 \mathrm{MHz}, \mathrm{CDCl}_{3}\right) \delta 1.97(\mathrm{~s}, 2 \mathrm{H}), 1.71(\mathrm{~s}, 3 \mathrm{H}), 1.28(\mathrm{~s}, 3$ $\mathrm{H}) ;{ }^{13} \mathrm{C}$ NMR $\left(75 \mathrm{MHz}, \mathrm{CDCl}_{3}\right) \delta 120.2,40.5,37.0,23.1,12.7,-3.4$. SAFETY NOTE: The distillation should be conducted using an efficient vacuum source at the lowest possible temperature. Although we encountered no difficulty conducting this distillation, differential scanning calorimetry analysis suggests that it is unsafe to heat this material above $100{ }^{\circ} \mathrm{C}$. 


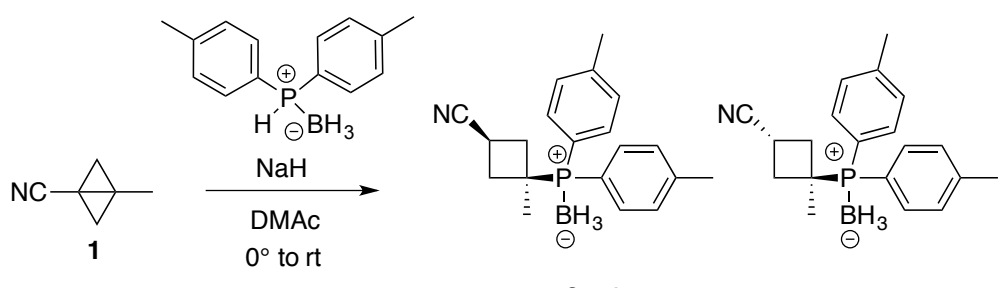

syn-2, $48 \% \quad$ anti-2, $28 \%$

3-(Di-p-tolylphosphanyl)-3-methylcyclobutane-1-carbonitrile borane 2.

A solution of $p$-tolylphosphine borane ${ }^{2}(0.500 \mathrm{~g}, 2.19 \mathrm{mmol})$ and bicyclobutane 1 (0.245 g, 2.63 $\mathrm{mmol})$ in DMAc $(10 \mathrm{~mL})$ was sparged with Ar for $10 \mathrm{~min}$, then cooled on ice. $\mathrm{NaH}(0.105 \mathrm{~g}, 2.63$ mmol) was added in one portion, affording a yellow solution that later faded to brown. The ice bath was removed, and the solution was stirred at $\mathrm{rt}$ for $1 \mathrm{~h}$. The reaction mixture was quenched with sat. aq. $\mathrm{NH}_{4} \mathrm{Cl}(5 \mathrm{~mL})$, and partitioned between water $(50 \mathrm{~mL})$ and ether $(50 \mathrm{~mL})$. The aqueous layer was extracted with ether $(30 \mathrm{~mL})$. The combined organic layers were washed with 1:1 water/brine $(50 \mathrm{~mL})$, dried $\left(\mathrm{Na}_{2} \mathrm{SO}_{4}\right)$ and concentrated. Chromatography on $\mathrm{SiO}_{2}(20-40 \%$ EtOAc/hexane) afforded syn-2 $(0.341 \mathrm{~g}, 1.06 \mathrm{mmol}, 48 \%)$ as a colorless solid and anti-2 (0.199 g,

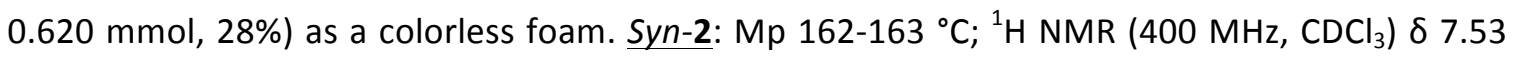
$(\mathrm{d}, 2 \mathrm{H}, J=8.4 \mathrm{~Hz}$ ), $7.51(\mathrm{~d}, 2 \mathrm{H}, J=8.0 \mathrm{~Hz}$ ), $7.29(\mathrm{~d}, 2 \mathrm{H}, J=8.0 \mathrm{~Hz}), 7.28(\mathrm{~d}, 2 \mathrm{H}, J=8.0 \mathrm{~Hz}$ ), 3.31 (pent, $1 \mathrm{H}, J=9.2 \mathrm{~Hz}$ ), 2.88-2.78 (m, $2 \mathrm{H}), 2.40(\mathrm{~s}, 6 \mathrm{H}), 2.34-2.26(\mathrm{~m}, 2 \mathrm{H}), 1.54\left(\mathrm{~d}, 3 \mathrm{H},{ }^{3} J_{\mathrm{PH}}=14.0\right.$ $\mathrm{Hz})$, 1.2-0.5 (br q, $\left.3 \mathrm{H}) ;{ }^{13} \mathrm{C} \mathrm{NMR} \mathrm{(125} \mathrm{MHz,} \mathrm{CDCl}\right) \delta 142.2\left(J_{\mathrm{CP}}=2 \mathrm{~Hz}\right), 133.4\left(J_{\mathrm{CP}}=7 \mathrm{~Hz}\right), 129.8$ $\left(J_{\mathrm{CP}}=8 \mathrm{~Hz}\right), 123.3,122.8,120.5\left({ }^{4} J_{\mathrm{CP}}=2 \mathrm{~Hz}\right), 34.7,33.4\left({ }^{1} J_{\mathrm{CP}}=34 \mathrm{~Hz}\right), 22.1\left(J_{\mathrm{CP}}=5 \mathrm{~Hz}\right), 21.5,18.2$ $\left({ }^{2} J_{\mathrm{CP}}=17 \mathrm{~Hz}\right) ;{ }^{31} \mathrm{P}$ NMR $\left(202 \mathrm{MHz}, \mathrm{CDCl}_{3}\right) \delta 27.7$; IR (ATR) 2392, 1429, 1066, $\left.807 \mathrm{~cm}^{-1} ; \mathrm{MS}(\mathrm{ESI})^{+}\right)$ $m / z$ 240, 219, 167, 139, 122; HRMS $\left(\mathrm{ESI}^{+}\right) \mathrm{m} / \mathrm{z}$ calcd for $\mathrm{C}_{20} \mathrm{H}_{26} \mathrm{BNP}[\mathrm{M}+\mathrm{H}]$ 322.1890, found 322.1879. Anti-2: ${ }^{1} \mathrm{H}$ NMR (400 MHz, $\left.\mathrm{CDCl}_{3}\right) \delta 7.51$ (d, $2 \mathrm{H}, J=8.4 \mathrm{~Hz}$ ), 7.49 (d, $\left.2 \mathrm{H}, J=8.0 \mathrm{~Hz}\right)$, $7.28(\mathrm{~d}, 2 \mathrm{H}, J=8.0 \mathrm{~Hz}$ ), 7.27 (d, $2 \mathrm{H}, J=8.0 \mathrm{~Hz}$ ), 3.07 (ddd, $2 \mathrm{H}, J=13.6 \mathrm{~Hz}, 13.6 \mathrm{~Hz}, 10.0 \mathrm{~Hz}$ ), 2.80 (ddd, $1 \mathrm{H}, J=17.0,9.6,7.6 \mathrm{~Hz}$ ), 2.46-2.36 (m, $2 \mathrm{H}$ ), $2.39(\mathrm{~s}, 6 \mathrm{H}), 1.60\left(\mathrm{~d}, 3 \mathrm{H},{ }^{3} J_{\mathrm{PH}}=12.8 \mathrm{~Hz}\right.$ ), 1.4-0.5 (br q, $3 \mathrm{H}) ;{ }^{13} \mathrm{C}$ NMR $\left(125 \mathrm{MHz}, \mathrm{CDCl}_{3}\right) \delta 142.10,142.08,133.2,133.1,129.8,129.7$, 123.6, 123.2, $122.1\left({ }^{4} J_{\mathrm{CP}}=0.5 \mathrm{~Hz}\right), 35.7,33.2\left({ }^{1} J_{\mathrm{CP}}=31 \mathrm{~Hz}\right), 25.7\left(J_{\mathrm{CP}}=1.5 \mathrm{~Hz}\right), 21.5\left(J_{\mathrm{CP}}=1.0 \mathrm{~Hz}\right)$, $16.9\left({ }^{2} J_{\mathrm{CP}}=6 \mathrm{~Hz}\right) ;{ }^{31} \mathrm{P}$ NMR $\left(202 \mathrm{MHz}, \mathrm{CDCl}_{3}\right) \delta 28.2$; IR (ATR) 2956, 2386, 1498, 1066, $814 \mathrm{~cm}^{-1}$;.

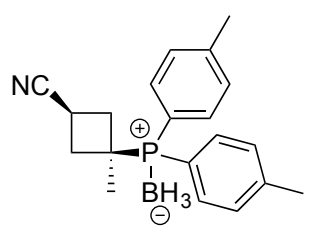

syn-2

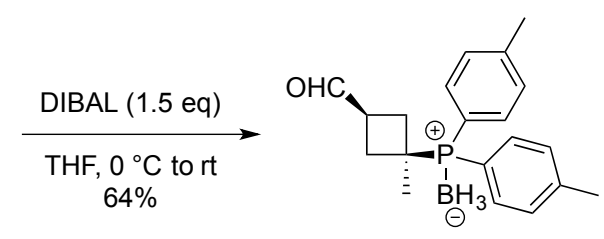

3 
3-(Di-p-tolylphosphanyl)-3-methylcyclobutane-1-carbaldehyde borane 3.

A solution of syn-2 $(0.755 \mathrm{~g}, 2.35 \mathrm{mmol})$ in THF (12 mL) was cooled to $0{ }^{\circ} \mathrm{C}$ under $\mathrm{N}_{2}$. DIBAL $(3.5$ $\mathrm{mL}, 3.5 \mathrm{mmol}, 1.5 \mathrm{eq}, 1 \mathrm{M}$ in hexanes) was added via syringe over $2 \mathrm{~min}$. The ice bath was removed, and the resulting solution was stirred at $\mathrm{rt}$ for $16 \mathrm{~h}$, then quenched with $2 \mathrm{M} \mathrm{H}_{2} \mathrm{SO}_{4}(10$ $\mathrm{mL}$ ). The solution was stirred for $1 \mathrm{~h}$ and gradually became an opaque white suspension. This suspension was partitioned between water $(40 \mathrm{~mL})$ and $\mathrm{Et}_{2} \mathrm{O}(40 \mathrm{~mL})$, and the aqueous layer was extracted with $\mathrm{Et}_{2} \mathrm{O}(40 \mathrm{~mL})$. The combined organic extracts were washed with water $(40 \mathrm{~mL})$ and brine $(40 \mathrm{~mL})$, dried $\left(\mathrm{Na}_{2} \mathrm{SO}_{4}\right)$ and concentrated. The crude product was purified by chromatography on $\mathrm{SiO}_{2}$ (20\% EtOAc in hexanes) to afford aldehyde 3 (0.484 g, $\left.1.49 \mathrm{mmol}, 64 \%\right)$ as a colorless foam: ${ }^{1} \mathrm{H} \mathrm{NMR}\left(400 \mathrm{MHz}, \mathrm{CDCl}_{3}\right) \delta 9.24(\mathrm{~d}, 1 \mathrm{H}, \mathrm{J}=2.0 \mathrm{~Hz}), 7.58(\mathrm{~d}, 2 \mathrm{H}, \mathrm{J}=8.4 \mathrm{~Hz})$, $7.55(\mathrm{~d}, 2 \mathrm{H}, J=8.4 \mathrm{~Hz}), 7.29(\mathrm{~d}, 2 \mathrm{H}, J=8.0 \mathrm{~Hz}), 7.28(\mathrm{~d}, 2 \mathrm{H}, J=7.6 \mathrm{~Hz}), 3.34-3.21(\mathrm{~m}, 1 \mathrm{H}, J=$ 9.2, 2.4 Hz), 2.88-2.74 (m, $2 \mathrm{H}), 2.41(\mathrm{~s}, 6 \mathrm{H}), 2.13-2.04(\mathrm{~m}, 2 \mathrm{H}), 1.61\left(\mathrm{~d}, 3 \mathrm{H},{ }^{3} \mathrm{JPH}_{\mathrm{PH}}=14.8 \mathrm{~Hz}\right), 1.2-$ 0.4 (br q, $3 \mathrm{H}) ;{ }^{13} \mathrm{C}$ NMR $\left(100 \mathrm{MHz} \mathrm{CDCl}_{3}\right) \delta 201.1,141.8,133.5,133.4,129.6,129.5,124.0$, 123.5, $40.3\left(J_{\mathrm{CP}}=12 \mathrm{~Hz}\right), 31.9\left({ }^{1} J_{\mathrm{CP}}=32 \mathrm{~Hz}\right), 30.6,23.6\left(J_{\mathrm{CP}}=5 \mathrm{~Hz}\right), 21.5 ;{ }^{31} \mathrm{P} \mathrm{NMR}(162 \mathrm{MHz}$, $\left.\mathrm{CDCl}_{3}\right) \delta$ 27.3; MS $\left(\mathrm{ESI}^{+}\right) \mathrm{m} / \mathrm{z} 324,311,295,213$; HRMS $\left(\mathrm{ESI}^{+}\right) \mathrm{m} / \mathrm{z}$ calcd for $\mathrm{C}_{20} \mathrm{H}_{27} \mathrm{BOP}[\mathrm{M}+\mathrm{H}]$ 325.1887 , found 325.1892 .

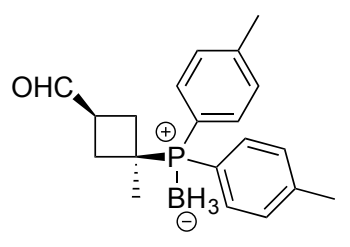

3

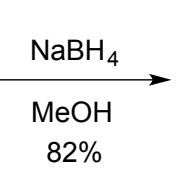

$82 \%$

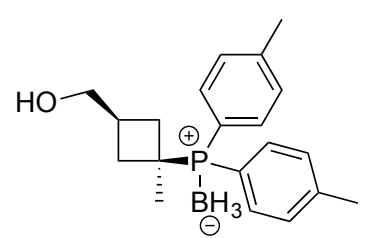

4

3-(Di-p-tolylphosphanyl)-3-methylcyclobutyl)methanol borane 4.

A solution of aldehyde $3(0.608 \mathrm{~g}, 1.88 \mathrm{mmol}, 1.00 \mathrm{eq})$ in $\mathrm{MeOH}(10 \mathrm{~mL})$ was cooled to $0{ }^{\circ} \mathrm{C}$. Sodium borohydride $(0.074 \mathrm{~g}, 2.0 \mathrm{mmol}, 1.1 \mathrm{eq})$ was added to the colorless solution in one portion. Bubbling ensued, and then subsided after ca. $10 \mathrm{~min}$. The reaction mixture was stirred at $\mathrm{rt}$ for $2 \mathrm{~h}$, and quenched with $1 \mathrm{M} \mathrm{HCl}(5 \mathrm{~mL})$. The contents of the flask were then partitioned between ether $(30 \mathrm{~mL})$ and water $(30 \mathrm{~mL})$, and the aqueous phase was extracted with ether (30 $\mathrm{mL})$. The combined organic phases were washed with water $(30 \mathrm{~mL})$ and brine $(30 \mathrm{~mL})$, were $\left(\mathrm{Na}_{2} \mathrm{SO}_{4}\right)$ and concentrated. The residue was purified by chromatography on $\mathrm{SiO}_{2}(25 \%$ EtOAc/hexane) to afford alcohol 4 (0.503 g, $1.54 \mathrm{mmol}, 82 \%)$ as a colorless solid: $\mathrm{Mp} 108-110^{\circ} \mathrm{C}$; 
${ }^{1} \mathrm{H}$ NMR $\left(400 \mathrm{MHz}, \mathrm{CDCl}_{3}\right) \delta 7.54(\mathrm{~d}, 2 \mathrm{H}, J=8.0 \mathrm{~Hz}), 7.52(\mathrm{~d}, 2 \mathrm{H}, J=8.8 \mathrm{~Hz}), 7.23(\mathrm{~d}, 4 \mathrm{H}, J=6.8$ $\mathrm{Hz}$ ), $3.19(\mathrm{~d}, 2 \mathrm{H}, J=6.4 \mathrm{~Hz}$ ), 2.74 (pent, $1 \mathrm{H}, J=8.0 \mathrm{~Hz}$ ), $2.37(\mathrm{~s}, 6 \mathrm{H}), 2.38-2.23(\mathrm{~m}, 2 \mathrm{H}), 2.01-$ $1.89(\mathrm{~m}, 2 \mathrm{H}), 1.54\left(\mathrm{~d}, 3 \mathrm{H},{ }^{3} J_{\mathrm{PH}}=14.8 \mathrm{~Hz}\right), 1.2-0.3(\mathrm{br} \mathrm{q}, 3 \mathrm{H}) ;{ }^{13} \mathrm{C} \mathrm{NMR}\left(100 \mathrm{MHz}, \mathrm{CDCl}_{3}\right) \delta$ 141.5, $141.5,133.5,133.5,129.4,129.3,124.7,124.2,66.2,33.1,32.2\left({ }^{2} J_{\mathrm{CP}}=13 \mathrm{~Hz}\right), 31.0\left({ }^{1} J_{\mathrm{CP}}=32 \mathrm{~Hz}\right)$, $23.5\left(J_{C P}=3 \mathrm{~Hz}\right), 21.5,14.2 ;{ }^{31} \mathrm{P}$ NMR $\left(162 \mathrm{MHz}, \mathrm{CDCl}_{3}\right) \delta 27.3$.

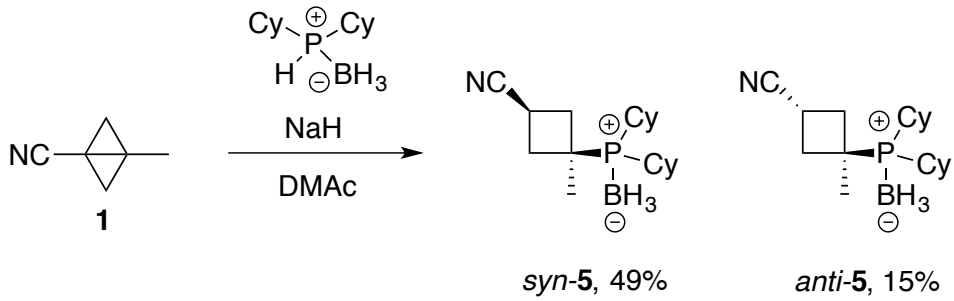

3-(Dicyclohexylphosphanyl)-3-methylcyclobutane-1-carbonitrile borane 5.

A solution of bicyclobutane $1(0.472 \mathrm{~g}, 5.07 \mathrm{mmol}, 1.2 \mathrm{eq})$ and dicyclohexyl phosphine borane $(0.896 \mathrm{~g}, 4.22 \mathrm{mmol})$ in DMAc $(20 \mathrm{~mL})$ was sparged with Ar for $10 \mathrm{~min}$, cooled to $0{ }^{\circ} \mathrm{C}$, and treated with $\mathrm{NaH}(0.203 \mathrm{~g}, 5.07 \mathrm{mmol}, 60 \%$ in oil) in one portion. The reaction mixture was stirred for $10 \mathrm{~min}$ at $0{ }^{\circ} \mathrm{C}$, then the bath was removed and the solution was warmed to rt over 2 h. The orange/brown reaction mixture was quenched with sat. aq. $\mathrm{NH}_{4} \mathrm{Cl}(5 \mathrm{~mL})$, and the material was partitioned between water $(30 \mathrm{~mL})$ and diethyl ether $(30 \mathrm{~mL})$. The aqueous layer was extracted with ether $(30 \mathrm{~mL})$. The combined extracts were washed with water $(40 \mathrm{~mL})$ and brine $(40 \mathrm{~mL})$, dried $\left(\mathrm{Na}_{2} \mathrm{SO}_{4}\right)$ and concentrated. The crude residue was purified by chromatography on $\mathrm{SiO}_{2}$ (15\% EtOAc/hexane) to afford syn-5 (0.634 g, $\left.2.08 \mathrm{mmol}, 49 \%\right)$ and anti-5 (0.197 g, $0.645 \mathrm{~g}, 15 \%)$, both as colorless solids. Syn-5: Mp 193-195 ${ }^{\circ} \mathrm{C} ;{ }^{1} \mathrm{H}$ NMR (400 MHz, $\mathrm{CDCl}_{3}$ ) $\delta 3.37$ (pent, $\left.1 \mathrm{H}, \mathrm{J}=9.6 \mathrm{~Hz}\right), 3.22-3.09(\mathrm{~m}, 2 \mathrm{H}), 2.08-2.01(\mathrm{~m}, 2 \mathrm{H}), 1.91-1.77(\mathrm{~m}, 8 \mathrm{H})$, 1.77-1.67 (br s, $2 \mathrm{H}), 1.66-1.58(\mathrm{~m}, 2 \mathrm{H}), 1.55\left(\mathrm{~d}, 3 \mathrm{H},{ }^{3} \mathrm{~J}_{\mathrm{PH}}=9.2 \mathrm{~Hz}\right), 1.48-1.31(\mathrm{~m}, 4 \mathrm{H}), 1.30-1.15$ (m, $6 \mathrm{H})$, 0.9-0.1 (br q, $3 \mathrm{H}) ;{ }^{31} \mathrm{P}$ NMR $\left(162 \mathrm{MHz}, \mathrm{CDCl}_{3}\right) \delta 35.8 ;{ }^{13} \mathrm{C} \mathrm{NMR}\left(125 \mathrm{MHz}, \mathrm{CDCl}_{3}\right) \delta 120.5$ $\left({ }^{4} J_{\mathrm{CP}}=2.5 \mathrm{~Hz}\right), 36.3,32.7\left({ }^{1} J_{\mathrm{CP}}=26 \mathrm{~Hz}\right), 31.5\left({ }^{1} J_{\mathrm{CP}}=29 \mathrm{~Hz}\right), 27.7$ (several overlapping resonances), 27.0, 25.9, 25.9, 22.1, 22.1, $19.7\left({ }^{1} J_{\mathrm{CP}}=17 \mathrm{~Hz}\right)$; IR (ATR) 2933, 2386, 1450, 1070, $840 \mathrm{~cm}^{-1}$; MS $\left(\mathrm{ESI}^{+}\right) \mathrm{m} / \mathrm{z}$ 306, 245, 215, 172; HRMS $\left(\mathrm{ESI}^{+}\right) \mathrm{m} / \mathrm{z}$ calcd for $\mathrm{C}_{18} \mathrm{H}_{34} \mathrm{BNP}[\mathrm{M}+\mathrm{H}]$ 306.2516, found 306.2506. Anti-5: Mp 165-168 ${ }^{\circ} \mathrm{C} ;{ }^{1} \mathrm{H}$ NMR $\left(400 \mathrm{MHz}, \mathrm{CDCl}_{3}\right) \delta 3.32-3.23(\mathrm{~m}, 1 \mathrm{H}), 3.11$ (dd, $2 \mathrm{H}, J$ $=10.8,2.4 \mathrm{~Hz}), 2.16-2.07(\mathrm{~m}, 2 \mathrm{H}), 1.88-1.75(\mathrm{~m}, 8 \mathrm{H}), 1.75-1.61(\mathrm{~m}, 2 \mathrm{H}), 1.67\left(\mathrm{~d}, 3 \mathrm{H},{ }^{3} \mathrm{JPH}_{\mathrm{PH}}=9.6\right.$ $\mathrm{Hz}), 1.47-1.32(\mathrm{~m}, 4 \mathrm{H}), 1.30-1.14(\mathrm{~m}, 7 \mathrm{H}), 0.7-0.0(\mathrm{br} \mathrm{q}, 3 \mathrm{H}) ;{ }^{31} \mathrm{P} \mathrm{NMR}\left(162 \mathrm{MHz}, \mathrm{CDCl}_{3}\right) \delta 34.3$; 
${ }^{13} \mathrm{C} \mathrm{NMR}\left(100 \mathrm{MHz}, \mathrm{CDCl}_{3}\right) \delta 122.9\left({ }^{4} J_{\mathrm{CP}}=1.0 \mathrm{~Hz}\right), 36.4,33.0\left({ }^{1} J_{\mathrm{CP}}=26 \mathrm{~Hz}\right), 31.3\left({ }^{1} J_{\mathrm{CP}}=29 \mathrm{~Hz}\right)$, 27.9, 27.1, 27.0, 27.0, 26.9, 25.8, $25.1\left(J_{\mathrm{CP}}=3 \mathrm{~Hz}\right), 17.8\left(J_{\mathrm{CP}}=9 \mathrm{~Hz}\right)$; IR (ATR) 2933, 2390, 1442, $1072,734 \mathrm{~cm}^{-1} ; \mathrm{HRMS}\left(\mathrm{ESI}^{+}\right) \mathrm{m} / z$ calcd for $\mathrm{C}_{18} \mathrm{H}_{34} \mathrm{BNP}[\mathrm{M}+\mathrm{H}] 306.2516$, found 306.2502 .

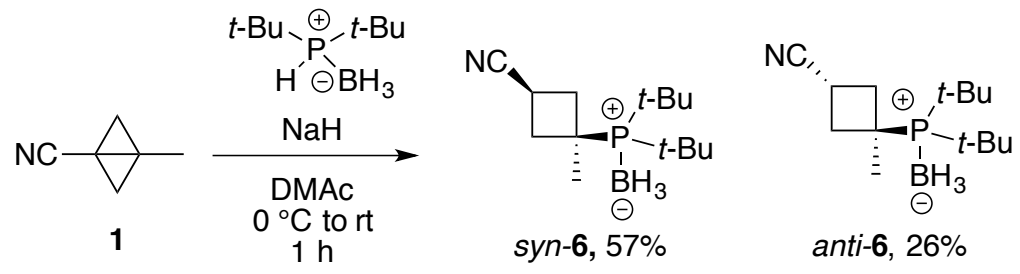

3-(Di-tert-butylphosphanyl)-3-methylcyclobutane-1-carbonitrile borane 6.

A solution of di-t-butyl phosphine borane $(0.363 \mathrm{~g}, 2.27 \mathrm{mmol})$ and bicyclobutane $1(0.264 \mathrm{~g}$, $2.84 \mathrm{mmol})$ in DMAc $(8 \mathrm{~mL})$ was sparged with Ar for $10 \mathrm{~min}$, then cooled to $0{ }^{\circ} \mathrm{C}$. NaH $(0.100 \mathrm{~g}$, $2.49 \mathrm{mmol}, 60 \%$ dispersion in oil) was added in one portion, causing foaming and the formation of a yellow/orange solution. After $5 \mathrm{~min}$, the cold bath was removed and the solution was warmed to rt over $1 \mathrm{~h}$. TLC (25\% EtOAc in hexane) analysis indicated consumption of starting material and formation of 2 more polar spots. The reaction mixture was quenched with sat. aq. $\mathrm{NH}_{4} \mathrm{Cl}(5 \mathrm{~mL})$ and partitioned between water $(50 \mathrm{~mL})$ and $\mathrm{Et}_{2} \mathrm{O}(35 \mathrm{~mL})$. The aqueous layer was extracted with $\mathrm{Et}_{2} \mathrm{O}(2 \times 30 \mathrm{~mL})$. The combined organic layers were washed with water $(30 \mathrm{~mL})$ and brine $(30 \mathrm{~mL})$, dried $\left(\mathrm{Na}_{2} \mathrm{SO}_{4}\right)$ and concentrated. Purification by chromatography on $\mathrm{SiO}_{2}$ (10-30\% EtOAc in hexanes) afforded syn-6 $(0.327 \mathrm{~g}, 1.29 \mathrm{mmol}, 57 \%)$ and anti-6 (0.147 g, 0.58 mmol, 26\%), both as colorless solids. Syn-6: $\mathrm{Mp} 125-127{ }^{\circ} \mathrm{C} ;{ }^{1} \mathrm{H} \mathrm{NMR}\left(400 \mathrm{MHz}, \mathrm{CDCl}_{3}\right) \delta 3.47-$ $3.31(\mathrm{~m}, 3 \mathrm{H}), 2.14-2.02(\mathrm{~m}, 2 \mathrm{H}), 1.83\left(\mathrm{~d}, 3 \mathrm{H},{ }^{3} \mathrm{~J}_{\mathrm{PH}}=8.8 \mathrm{~Hz}\right), 1.33(\mathrm{~s}, 9 \mathrm{H}), 1.30(\mathrm{~s}, 9 \mathrm{H}), 0.61$ (br q, $\left.3 \mathrm{H},{ }^{1} J_{\mathrm{BH}}=98 \mathrm{~Hz}\right) ;{ }^{13} \mathrm{C} \mathrm{NMR}\left(100 \mathrm{MHz}, \mathrm{CDCl}_{3}\right) \delta 120.6\left({ }^{4} J_{\mathrm{CP}}=3 \mathrm{~Hz}\right), 38.1,35.8\left({ }^{1} J_{\mathrm{CP}}=20 \mathrm{~Hz}\right), 35.1$ $\left({ }^{1} J_{\mathrm{CP}}=23 \mathrm{~Hz}\right), 29.6,23.7\left(J_{\mathrm{CP}}=4 \mathrm{~Hz}\right), 20.1\left(J_{\mathrm{CP}}=18 \mathrm{~Hz}\right) ;{ }^{31} \mathrm{P} \mathrm{NMR}\left(162 \mathrm{MHz}, \mathrm{CDCl}_{3}\right) \delta 54.7$; IR (ATR) 2974, 2378, 2235, 1480, $1072 \mathrm{~cm}^{-1}$; MS (ESI $\left.{ }^{+}\right) \mathrm{m} / \mathrm{z}$ 240, 223, 208, 196, 162, 142, 114; HRMS (ESI ${ }^{+}$) $\mathrm{m} / \mathrm{z}$ calcd for $\mathrm{C}_{14} \mathrm{H}_{30} \mathrm{BNP}[\mathrm{M}+\mathrm{H}]^{+} 254.2203$, found 254.2197. Anti-6: Mp 53-56 ${ }^{\circ} \mathrm{C}$; ${ }^{1} \mathrm{H}$ NMR (400 $\mathrm{MHz}, \mathrm{CDCl}_{3}$ ) $\delta 3.48\left(\operatorname{app~q}, 2 \mathrm{H}, J=12.4 \mathrm{~Hz}\right.$ ), 3.27-3.17 (m, $1 \mathrm{H}$ ), $2.09\left(\mathrm{~d}, 3 \mathrm{H},{ }^{3} \mathrm{~J}_{\mathrm{PH}}=9.2\right), 2.13-2.05$ $(\mathrm{m}, 2 \mathrm{H}), 1.35(\mathrm{~s}, 9 \mathrm{H}), 1.32(\mathrm{~s}, 9 \mathrm{H}), 0.53\left(\mathrm{br} \mathrm{q}, 3 \mathrm{H},{ }^{1} J_{\mathrm{BH}}=98 \mathrm{~Hz}\right) ;{ }^{13} \mathrm{C} \mathrm{NMR}\left(100 \mathrm{MHz}, \mathrm{CDCl}_{3}\right) \delta$ 123.6, 123.5, 37.5, $36.9\left({ }^{1} J_{\mathrm{CP}}=20 \mathrm{~Hz}\right), 35.1\left({ }^{1} J_{\mathrm{CP}}=23 \mathrm{~Hz}\right), 29.6,26.5\left(J_{\mathrm{CP}}=4 \mathrm{~Hz}\right), 19.3\left(J_{\mathrm{CP}}=15 \mathrm{~Hz}\right)$; ${ }^{31} \mathrm{P}$ NMR $\left(162 \mathrm{MHz}, \mathrm{CDCl}_{3}\right) \delta 53.6$. 


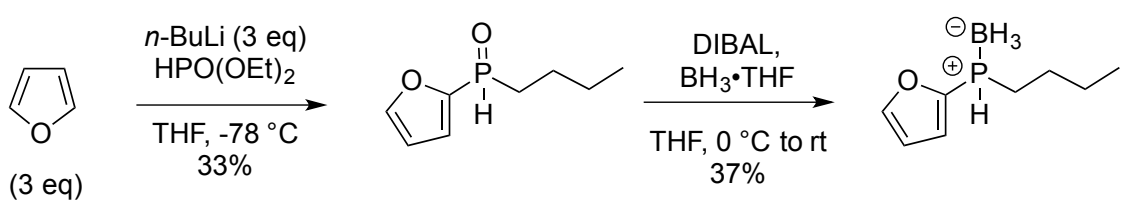

Butyl(furan-2-yl)(methyl)phosphane borane.

A $250 \mathrm{~mL}$ flask was charged with a solution of furan $(4.7 \mathrm{~mL}, 65.1 \mathrm{mmol})$ in THF $(80 \mathrm{~mL})$ under $\mathrm{N}_{2}$. The solution was cooled to $-78^{\circ} \mathrm{C}$, then $n$-butyllithium $(26 \mathrm{~mL}, 66 \mathrm{mmol}, 2.5 \mathrm{M}$ in hexane) was added via syringe over $45 \mathrm{~min}$. The reaction mixture was stirred for $30 \mathrm{~min}$ at $-78{ }^{\circ} \mathrm{C}$, then diethyl phosphite $(2.8 \mathrm{~mL}, 22 \mathrm{mmol}$ ) was added via syringe over $15 \mathrm{~min}$. The solution was warmed to $\mathrm{rt}$, and a ${ }^{31} \mathrm{P}$ NMR of an aliquot showed full consumption of diethyl phosphite (phosphite signal at $6 \mathrm{ppm}$, product signal at $11 \mathrm{ppm}$ ). The reaction mixture was quenched with $0.1 \mathrm{~N} \mathrm{HCl}(120 \mathrm{~mL})$ at $0{ }^{\circ} \mathrm{C}$, followed by addition of $\mathrm{Et}_{2} \mathrm{O}(120 \mathrm{~mL})$ and stirred for an additional 5 $\min$. The aqueous phase was extracted with $\mathrm{Et}_{2} \mathrm{O}(75 \mathrm{~mL})$ and the combined extracts were washed with water $(100 \mathrm{~mL})$, brine $(100 \mathrm{~mL})$, and concentrated. Chromatography on $\mathrm{SiO}_{2}(50-$ $100 \%$ EtOAc in hexane) afforded furyl $n$-butyl phosphine oxide $(1.25 \mathrm{~g}, 6.85 \mathrm{mmol}, 33 \%)$ as a colorless oil: ${ }^{1} \mathrm{H}$ NMR $\left(400 \mathrm{MHz}, \mathrm{CDCl}_{3}\right) \delta 7.68(\mathrm{~s}, 1 \mathrm{H}), 7.52\left(\mathrm{dt}, 1 \mathrm{H},{ }^{1} \mathrm{~J}_{\mathrm{PH}}=484 \mathrm{~Hz}, J=3.2 \mathrm{~Hz}\right), 7.14$ (s, $1 \mathrm{H}), 6.52(\mathrm{t}, 1 \mathrm{H}, \mathrm{J}=1.6 \mathrm{~Hz}), 2.14-2.05(\mathrm{~m}, 2 \mathrm{H}), 1.68-1.55(\mathrm{~m}, 2 \mathrm{H}), 1.50-1.37(\mathrm{~m}, 2 \mathrm{H}), 0.92(\mathrm{t}$, $3 \mathrm{H}, J=7.6 \mathrm{~Hz}) ;{ }^{31} \mathrm{P}$ NMR $\left(202 \mathrm{MHz}, \mathrm{CDCl}_{3}\right) \delta 11.8$.

A 3-necked $100 \mathrm{~mL}$ flask equipped with a thermometer, septum, and $\mathrm{N}_{2}$ inlet was charged with DIBAL ( $22 \mathrm{~mL}, 20 \mathrm{mmol}, 0.92 \mathrm{M}$ in THF). The mixture was cooled to an internal temperature of 2 ${ }^{\circ} \mathrm{C}$ on an ice bath. A solution of phosphine oxide $(1.24 \mathrm{~g}, 6.81 \mathrm{mmol})$ in THF (10 mL) was added by syringe over $20 \mathrm{~min}$, causing a maximum exotherm of $5{ }^{\circ} \mathrm{C}$. The resulting green-tinted solution was stirred at $2{ }^{\circ} \mathrm{C}$ for $1 \mathrm{~h}$, then $\mathrm{BH}_{3} / \mathrm{THF}(6.8 \mathrm{~mL}, 6.8 \mathrm{mmol}, 1.0 \mathrm{M}$ in THF) was added via syringe. The cooling bath was removed, and the clear, colorless solution was stirred at rt for 21 h. The solution was put on ice and quenched slowly with $1 \mathrm{M} \mathrm{NaOH}(40 \mathrm{~mL}$, maximum exotherm $\left.35{ }^{\circ} \mathrm{C}\right)$, then diluted with ether $(50 \mathrm{~mL})$. The biphasic mixture was filtered through Celite to remove aluminum salts, then the layers were separated. The aqueous layer was extracted with ether $(2 \times 40 \mathrm{~mL})$. The combined extracts were washed with water $(60 \mathrm{~mL})$ and brine $(150 \mathrm{~mL})$, dried $\left(\mathrm{Na}_{2} \mathrm{SO}_{4}\right)$, and filtered through Celite. The colorless filtrate was by chromatography on $\mathrm{SiO}_{2}$ (10\% EtOAc/hexanes) to afford furyl $n$-butyl phosphine borane $(0.453 \mathrm{~g}, 2.66 \mathrm{mmol}, 37 \%)$ as a colorless oil: ${ }^{1} \mathrm{H}$ NMR $\left(400 \mathrm{MHz}, \mathrm{CDCl}_{3}\right) \delta 7.72(\mathrm{~s}, 1 \mathrm{H}), 7.12$ (tdd, $1 \mathrm{H}, 2.0 \mathrm{~Hz}, 1.2 \mathrm{~Hz}, 0.8 \mathrm{~Hz}$ ), 6.51 
(ddd, $1 \mathrm{H}, J=3.2 \mathrm{~Hz}, 1.6 \mathrm{~Hz}, 1.6 \mathrm{~Hz}$ ), 5.57 (d sextet, $1 \mathrm{H},{ }^{1} J_{\mathrm{PH}}=376 \mathrm{~Hz}, J=1.5 \mathrm{~Hz}$ ), 2.08-1.89 (m, 2 $\mathrm{H}), 1.70-1.48(\mathrm{~m}, 2 \mathrm{H}), 1.47-1.36(\mathrm{~m}, 2 \mathrm{H}), 0.92(\mathrm{t}, 3 \mathrm{H}, J=7.6 \mathrm{~Hz})$ 1.3-0.4 (br q, $3 \mathrm{H}) ;{ }^{31} \mathrm{P} N M R$ $\left(202 \mathrm{MHz}, \mathrm{CDCl}_{3}\right) \delta-25.9 ;{ }^{13} \mathrm{C} \mathrm{NMR}\left(100 \mathrm{MHz}, \mathrm{CDCl}_{3}\right) \delta 148.8\left({ }^{3} \mathrm{JP}_{\mathrm{CP}}=4 \mathrm{~Hz}\right), 141.9\left({ }^{1} \mathrm{JP}_{\mathrm{CP}}=66 \mathrm{~Hz}\right)$, $124.1\left({ }^{2} J_{\mathrm{CP}}=20 \mathrm{~Hz}\right), 111.3\left({ }^{3} J_{\mathrm{CP}}=8 \mathrm{~Hz}\right), 26.1\left({ }^{3} J_{\mathrm{CP}}=2 \mathrm{~Hz}\right), 23.7\left({ }^{2} J_{\mathrm{CP}}=13 \mathrm{~Hz}\right), 21.3\left({ }^{1} J_{\mathrm{CP}}=38 \mathrm{~Hz}\right)$, 13.5.

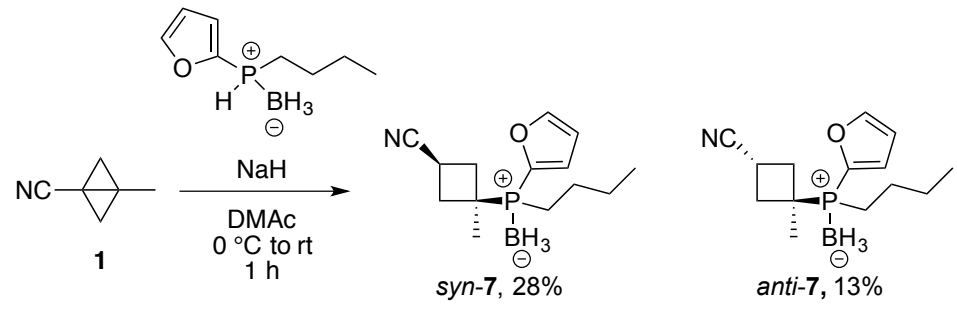

3-(Butyl(furan-2-yl)phosphanyl)-3-methylcyclobutane-1-carbonitrile borane 7.

A solution of racemic furyl butyl phosphine borane $(0.261 \mathrm{~g}, 1.54 \mathrm{mmol})$ and bicyclobutane 1 (0.172 g, $1.8 \mathrm{mmol}, 1.20 \mathrm{eq})$ in DMAc (8 mL) was sparged with Ar for $10 \mathrm{~min}$ in a $25 \mathrm{~mL}$ flask, then it was cooled on ice. $\mathrm{NaH}(0.074 \mathrm{~g}, 1.8 \mathrm{mmol}, 1.2 \mathrm{eq}, 60 \%$ in oil) was added in one portion, affording a yellow solution. The ice bath was removed, and the solution was stirred at $\mathrm{rt}$ for $1 \mathrm{~h}$ and faded to a brown color. The mixture was quenched with sat. aq. $\mathrm{NH}_{4} \mathrm{Cl}(5 \mathrm{~mL})$, partitioned between water $(30 \mathrm{~mL})$ and ether $(30 \mathrm{~mL})$, and the aqueous layer was extracted with ether $(50$ $\mathrm{mL})$. The combined organic layers were washed with 1:1 water/brine $(50 \mathrm{~mL})$, dried $\left(\mathrm{Na}_{2} \mathrm{SO}_{4}\right)$ and concentrated. Chromatography on $\mathrm{SiO}_{2}$ (15-40\% EtOAc/hexane) afforded syn-7 (0.115 g, $0.437 \mathrm{mmol}, 28 \%)$ and anti-7 (0.052 g, $0.20 \mathrm{mmol}, 13 \%)$, both as colorless oils. Syn-7: ${ }^{1} \mathrm{H}$ NMR (400 MHz, $\left.\mathrm{CDCl}_{3}\right) \delta 7.74(\mathrm{~s}, 1 \mathrm{H}), 7.18-7.15(\mathrm{~m}, 1 \mathrm{H}), 6.53-6.50(\mathrm{~m}, 1 \mathrm{H}), 3.28$ (pent, $1 \mathrm{H}, J=9.2$ Hz), 2.88 (td, 1 H, 13.2 Hz, 9.2 Hz), 2.69 (td, 1 H, 13.6 Hz, 9.2 Hz), 2.19-1.98 (m, 3 H), 1.77-1.66 (m, $1 \mathrm{H}), 1.60-1.47(\mathrm{~m}, 1 \mathrm{H}), 1.44\left(\mathrm{~d}, 3 \mathrm{H},{ }^{3} J_{\mathrm{PH}}=14.4 \mathrm{~Hz}\right.$ ), 1.35 (sextet, $2 \mathrm{H}, J=7.2 \mathrm{~Hz}$ ), 1.28-1.13 $(\mathrm{m}, 1 \mathrm{H}), 0.87(\mathrm{t}, 3 \mathrm{H}, J=7.2 \mathrm{~Hz}), 1.1-0.0(\mathrm{br} \mathrm{q}, 3 \mathrm{H}) ;{ }^{13} \mathrm{C} \mathrm{NMR}\left(100 \mathrm{MHz}, \mathrm{CDCl}_{3}\right) \delta 149.1\left({ }^{3} \mathrm{JP}_{\mathrm{CP}}=3\right.$ $\mathrm{Hz}), 143.2\left({ }^{1} J_{\mathrm{CP}}=61 \mathrm{~Hz}\right), 125.8\left({ }^{2} J_{\mathrm{CP}}=18 \mathrm{~Hz}\right), 121.1\left({ }^{4} J_{\mathrm{CP}}=2 \mathrm{~Hz}\right), 111.3\left({ }^{3} J_{\mathrm{CP}}=8 \mathrm{~Hz}\right), 34.1,33.9$, $32.7\left({ }^{1} J_{\mathrm{CP}}=35 \mathrm{~Hz}\right), 25.0,24.1\left({ }^{2} J_{\mathrm{CP}}=14 \mathrm{~Hz}\right), 22.1,\left({ }^{3} J_{\mathrm{CP}}=3 \mathrm{~Hz}\right), 19.0\left({ }^{1} J_{\mathrm{CP}}=36 \mathrm{~Hz}\right), 17.1\left({ }^{2} J_{\mathrm{CP}}=16\right.$ $\mathrm{Hz}), 13.6 ;{ }^{31} \mathrm{P} N M R\left(162 \mathrm{MHz}, \mathrm{CDCl}_{3}\right) \delta$ 18.6; IR (ATR) 2956, 2933, 2386, 2240, 1456, 1128, 1072, $757 \mathrm{~cm}^{-1} ; \mathrm{MS}\left(\mathrm{ESI}^{+}\right) \mathrm{m} / \mathrm{z}$ 264, 250, 228, 200, 184, 154, 110; HRMS (ESI $\left.{ }^{+}\right) \mathrm{m} / \mathrm{z}$ calcd for $\mathrm{C}_{14} \mathrm{H}_{24} \mathrm{BNOP}$ $[\mathrm{M}+\mathrm{H}]$ 264.1683, found 264.1685. Anti-7: ${ }^{1} \mathrm{H}$ NMR (400 MHz, CDCl $) \delta 7.71(\mathrm{~s}, 1 \mathrm{H}), 7.16(\mathrm{~d}, 1 \mathrm{H}, \mathrm{J}$ 
$=1.2 \mathrm{~Hz}$ ), $6.51(\mathrm{t}, 1 \mathrm{H}, J=1.6 \mathrm{~Hz}$ ), 2.98 (pent, $1 \mathrm{H}, J=7.6 \mathrm{~Hz}), 2.89-2.77(\mathrm{~m}, 2 \mathrm{H}), 2.32-2.12(\mathrm{~m}, 2$ H), 2.08 (dd, $1 \mathrm{H}, J=14.8,4.0 \mathrm{~Hz}$ ), 2.01 (dd, $1 \mathrm{H}, J=14.8,4.0 \mathrm{~Hz}$ ), 1.76-1.63 (m, $1 \mathrm{H}$ ), 1.58-1.44 $(\mathrm{m}, 1 \mathrm{H}), 1.53\left(\mathrm{~d}, 3 \mathrm{H},{ }^{3} J_{\mathrm{PH}}=12.0 \mathrm{~Hz}\right), 1.35$ (pent, $\left.2 \mathrm{H}, J=7.2 \mathrm{~Hz}\right), 1.29-1.18(\mathrm{~m}, 1 \mathrm{H}), 0.86(\mathrm{t}, 3 \mathrm{H}, J$ = 7.2 Hz), 1.1-0.1 (br q, $3 \mathrm{H}) ;{ }^{13} \mathrm{C} \mathrm{NMR}\left(100 \mathrm{MHz}, \mathrm{CDCl}_{3}\right) \delta 148.9\left({ }^{3} \mathrm{~J}_{\mathrm{CP}}=3 \mathrm{~Hz}\right), 143.2\left({ }^{1} \mathrm{~J}_{\mathrm{CP}}=61 \mathrm{~Hz}\right)$, $125.5\left({ }^{2} J_{\mathrm{CP}}=18 \mathrm{~Hz}\right), 122.1,111.4\left({ }^{3} J_{\mathrm{CP}}=8 \mathrm{~Hz}\right), 35.0,34.9\left({ }^{3} J_{\mathrm{CP}}=3 \mathrm{~Hz}\right), 33.2\left({ }^{1} J_{\mathrm{CP}}=33 \mathrm{~Hz}\right), 25.0$, $24.1\left({ }^{2} J_{\mathrm{CP}}=13 \mathrm{~Hz}\right), 18.8\left({ }^{1} J_{\mathrm{CP}}=36 \mathrm{~Hz}\right), 16.2\left({ }^{3} \mathrm{~J}_{\mathrm{CP}}=5 \mathrm{~Hz}\right), 13.6 ;{ }^{31} \mathrm{P} \mathrm{NMR}\left(202 \mathrm{MHz}, \mathrm{CDCl}_{3}\right) \delta 17.6^{\prime} \mathrm{IR}$ (ATR) 2958, 2380, 2238, 1555, 1458, 1212, 1128, 1071, 1049, 1007, 909, $732 \mathrm{~cm}^{-1}$

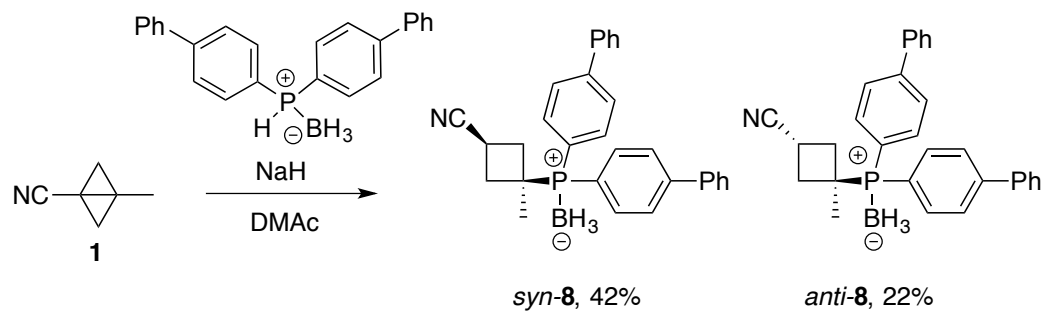

3-(Di([1,1'-biphenyl]-4-yl)phosphanyl)-3-methylcyclobutane-1-carbonitrile borane 8.

A solution of dibiphenylphosphine borane $(0.300 \mathrm{~g}, 0.850 \mathrm{mmol})$ and bicyclobutane 1 (0.095 $\mathrm{g}$, $1.0 \mathrm{mmol}$ ) in DMAc (4 mL) was sparged with Ar for $10 \mathrm{~min}$, then cooled in an ice bath. $\mathrm{NaH}$ $(0.036 \mathrm{~g}, 0.89 \mathrm{mmol})$ was added in one portion, affording a yellow solution that later faded to brown. The ice bath was removed, and the solution was stirred at $\mathrm{rt}$ for $2 \mathrm{~h}$, quenched with sat. aq. $\mathrm{NH}_{4} \mathrm{Cl}(5 \mathrm{~mL})$, and partitioned between water $(50 \mathrm{~mL})$ and ether $(50 \mathrm{~mL})$. The aqueous layer was extracted with ether $(30 \mathrm{~mL})$. The combined organic layers were washed with 1:1 water/brine $(50 \mathrm{~mL})$, dried $\left(\mathrm{Na}_{2} \mathrm{SO}_{4}\right)$, and concentrated. Chromatography on $\mathrm{SiO}_{2}(15-30 \%$ EtOAc/hexane) afforded syn-8 (0.158 g, $0.35 \mathrm{mmol}, 42 \%)$ and anti-8 (0.083 g, $0.186 \mathrm{mmol}, 22 \%)$, both as sticky waxes. Syn-8: ${ }^{1} \mathrm{H}$ NMR $\left(400 \mathrm{MHz}, \mathrm{CDCl}_{3}\right) \delta$ 7.79-7.70 (m, $\left.8 \mathrm{H}\right)$, 7.64-7.59 (m, $\left.4 \mathrm{H}\right)$, $7.48(\mathrm{t}, 4 \mathrm{H}, J=7.5 \mathrm{~Hz}$ ), 7.40 (t, $2 \mathrm{H}, J=7.2 \mathrm{~Hz}$ ), 3.38 (pent, $1 \mathrm{H}, J=9.2 \mathrm{~Hz}$ ), 2.94 (dd, $2 \mathrm{H}, J=12.8$ $\mathrm{Hz}, 9.6 \mathrm{~Hz}), 2.42-2.35(\mathrm{~m}, 2 \mathrm{H}), 1.61\left(\mathrm{~d}, 3 \mathrm{H},{ }^{3} J_{\mathrm{PH}}=14.4 \mathrm{~Hz}\right), 1.4-0.7(\mathrm{br} \mathrm{q}, 3 \mathrm{H}) ;{ }^{13} \mathrm{C} N M R(100$ $\left.\mathrm{MHz}, \mathrm{CDCl}_{3}\right) \delta 144.6\left(J_{\mathrm{CP}}=2 \mathrm{~Hz}\right), 139.7,134.0,129.1,128.3,127.7,127.3,125.3,124.7,120.5$, 34.8, 33.5 $\left({ }^{1} J_{\mathrm{CP}}=33 \mathrm{~Hz}\right), 22.3\left(J_{\mathrm{CP}}=5 \mathrm{~Hz}\right), 18.3\left({ }^{2} J_{\mathrm{CP}}=17 \mathrm{~Hz}\right) ;{ }^{31} \mathrm{P} \mathrm{NMR}\left(202 \mathrm{MHz}, \mathrm{CDCl}_{3}\right) \delta$ 29.0; IR (ATR) 3027, 2384, 1077, 911, $726 \mathrm{~cm}^{-1}$; MS $\left(\mathrm{ESI}^{+}\right) \mathrm{m} / \mathrm{z}$ 423, 324, 292, 224, 124; HRMS (ESI $) \mathrm{m} / \mathrm{z}$ calcd for $\mathrm{C}_{30} \mathrm{H}_{30} \mathrm{BNP}[\mathrm{M}+\mathrm{H}]$ 446.2203, found 446.2211. Anti-8: ${ }^{1} \mathrm{H}$ NMR $\left(400 \mathrm{MHz}, \mathrm{CDCl}_{3}\right) \delta 7.76-$ $7.68(\mathrm{~m}, 8 \mathrm{H}), 7.61(\mathrm{~d}, 4 \mathrm{H}, J=7.5 \mathrm{~Hz}), 7.49(\mathrm{t}, 4 \mathrm{H}, J=7.5 \mathrm{~Hz}), 7.42(\mathrm{t}, 2 \mathrm{H}, J=7.5 \mathrm{~Hz}), 3.20$ (dd, 2 
$\mathrm{H}, J=13.6 \mathrm{~Hz}, 10.0 \mathrm{~Hz}), 3.03-2.90(\mathrm{~m}, 1 \mathrm{H}), 2.49(\mathrm{td}, 2 \mathrm{H}, J=14.0 \mathrm{~Hz}, 7.0 \mathrm{~Hz}), 1.71\left(\mathrm{~d}, 3 \mathrm{H},{ }^{3} J_{\mathrm{PH}}=\right.$ 12.8 Hz), 1.4-0.7 (br m, $3 \mathrm{H}) ;{ }^{13} \mathrm{C} \mathrm{NMR}\left(100 \mathrm{MHz} \mathrm{CDCl}_{3}\right) \delta 144.5\left(J_{\mathrm{CP}}=2 \mathrm{~Hz}\right), 139.6,133.8,129.1$, $128.4,127.6,127.2,125.5,125.0,122.1,35.7,33.4\left({ }^{1} J_{\mathrm{CP}}=31 \mathrm{~Hz}\right), 25.8,17.1\left({ }^{2} J_{\mathrm{CP}}=6 \mathrm{~Hz}\right) ;{ }^{31} \mathrm{P} N M R$ $\left(202 \mathrm{MHz}, \mathrm{CDCl}_{3}\right.$ ) $\delta$ 29.1; IR (ATR) 3029, 2980, 2374, 2236, 1597, 1483, 1389, 1109, 1061, 1006, $909,834,736,695 \mathrm{~cm}^{-1}$
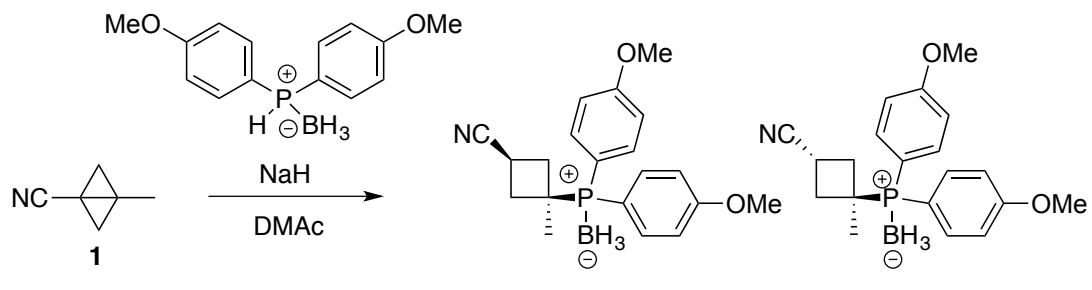

syn- $9,43 \%$

anti-9, $16 \%$

3-(Bis(4-methoxyphenyl)phosphanyl)-3-methylcyclobutane-1-carbonitrile borane 9.

A solution of di-p-methoxy phosphine borane ${ }^{3}(0.222 \mathrm{~g}, 0.854 \mathrm{mmol})$ and bicyclobutane 1 (0.254 $\mathrm{g}, 2.73 \mathrm{mmol})$ in DMAc $(6 \mathrm{~mL})$ was sparged with Ar for $15 \mathrm{~min}$, then cooled to $0{ }^{\circ} \mathrm{C}$. NaH $(0.038 \mathrm{~g}$, $0.94 \mathrm{mmol}$ ) was added, and the reaction mixture was warmed to $\mathrm{rt}$, stirred for $1.5 \mathrm{~h}$, and quenched with sat aq $\mathrm{NH}_{4} \mathrm{Cl}(3 \mathrm{~mL})$. The solution was partitioned between water and diethyl ether (30 $\mathrm{mL}$ each), and the aqueous phase was extracted with ether $(30 \mathrm{~mL})$. The combined organic extracts were washed with water $(30 \mathrm{~mL})$, and brine $(30 \mathrm{~mL})$, dried $\left(\mathrm{Na}_{2} \mathrm{SO}_{4}\right)$ and concentrated. Purification by chromatography on $\mathrm{SiO}_{2}$ (10-30\% EtOAc/hexane) afforded recovered 1 (21 mg), anti-9 (0.048 g, $0.14 \mathrm{mmol}, 16 \%)$ as a colorless oil, and syn-9 (0.130 g, $0.368 \mathrm{mmol}, 43 \%)$ as a colorless foam. Syn-9: ${ }^{1} \mathrm{H} \mathrm{NMR}\left(400 \mathrm{MHz}, \mathrm{CDCl}_{3}\right) \delta 7.57(\mathrm{t}, 4 \mathrm{H}, \mathrm{J}=9.2 \mathrm{~Hz})$, 6.99 (dd, $4 \mathrm{H}, J=8.8 \mathrm{~Hz}, 1.6 \mathrm{~Hz}$ ), $3.84(\mathrm{~s}, 6 \mathrm{H}$ ), 3.31 (pent, $1 \mathrm{H}, J=9.2 \mathrm{~Hz}$ ), 2.79 (qd, $2 \mathrm{H}, J=10.0$ $\mathrm{Hz}, 2.0 \mathrm{~Hz}), 2.34-2.24(\mathrm{~m}, 2 \mathrm{H}), 1.50\left(\mathrm{~d}, 3 \mathrm{H},{ }^{3} \mathrm{JPH}_{\mathrm{PH}}=14.4 \mathrm{~Hz}\right), 1.2-0.3(\mathrm{br} \mathrm{m}, 3 \mathrm{H}) ;{ }^{13} \mathrm{C} N M R(100$ $\left.\mathrm{MHz}, \mathrm{CDCl}_{3}\right) \delta 162.23,162.21,135.1,135.0,120.6\left({ }^{4} J_{\mathrm{CP}}=2 \mathrm{~Hz}\right), 117.6,117.0,114.7,114.6,55.4$, 34.6, $33.6\left({ }^{1} J_{\mathrm{CP}}=34 \mathrm{~Hz}\right), 22.1\left(J_{\mathrm{CP}}=5 \mathrm{~Hz}\right), 18.2\left(J_{\mathrm{CP}}=17 \mathrm{~Hz}\right) ;{ }^{31} \mathrm{P} \mathrm{NMR}\left(162 \mathrm{MHz}, \mathrm{CDCl}_{3}\right) \delta 26.2 ; \mathrm{IR}$ (ATR) 2394, 2385, 1600, 1499, 1245, 1109, 1021, $798 \mathrm{~cm}^{-1}$; MS (ESI $\left.{ }^{+}\right) \mathrm{m} / \mathrm{z} 318,316,259,247$; HRMS $\left(\mathrm{ESI}^{+}\right) \mathrm{m} / \mathrm{z}$ calcd for $\mathrm{C}_{20} \mathrm{H}_{26} \mathrm{BNO}_{2} \mathrm{P}[\mathrm{M}+\mathrm{H}]^{+}$354.1789, found 354.1793. Anti-9: ${ }^{1} \mathrm{H}$ NMR (400 $\left.\mathrm{MHz}, \mathrm{CDCl}_{3}\right) \delta 7.53(\mathrm{tt}, 4 \mathrm{H}, J=9.2 \mathrm{~Hz}, 2.0 \mathrm{~Hz}), 6.96(\mathrm{dd}, 4 \mathrm{H}, J=8.8 \mathrm{~Hz}, 1.6 \mathrm{~Hz}), 3.84(\mathrm{~s}, 6 \mathrm{H}), 3.04$ (td, $2 \mathrm{H}, 13.6 \mathrm{~Hz}, 10.0 \mathrm{~Hz}$ ), 2.74 (ddd, $1 \mathrm{H}, J=17.6 \mathrm{~Hz}, 9.6 \mathrm{~Hz}, 7.6 \mathrm{~Hz}), 2.39(\mathrm{td}, 2 \mathrm{H}, J=13.2 \mathrm{~Hz}$, $7.6 \mathrm{~Hz}$ ), 1.59 (d, $\left.3 \mathrm{H},{ }^{3} \mathrm{~J}_{\mathrm{PH}}=12.8 \mathrm{~Hz}\right), 1.4-0.5(\mathrm{br} \mathrm{m}, 3 \mathrm{H}) ;{ }^{13} \mathrm{C} \mathrm{NMR}\left(100 \mathrm{MHz}, \mathrm{CDCl}_{3}\right) \delta$ 162.09, $162.07,134.8,134.7,122.1,117.8,117.2,114.6,114.5,55.3,35.6,33.4\left({ }^{1} J_{C P}=32 \mathrm{~Hz}\right), 25.7\left(J_{C P}=\right.$ 
$2 \mathrm{~Hz}), 16.8\left(J_{\mathrm{CP}}=6 \mathrm{~Hz}\right) ;{ }^{31} \mathrm{P}$ NMR $\left(162 \mathrm{MHz}, \mathrm{CDCl}_{3}\right) \delta$ 26.3; IR (ATR) 2940, 2379, 2237, 1902, 1594, $1501,1289,1253,1180,1061,1024,801 \mathrm{~cm}^{-1}$

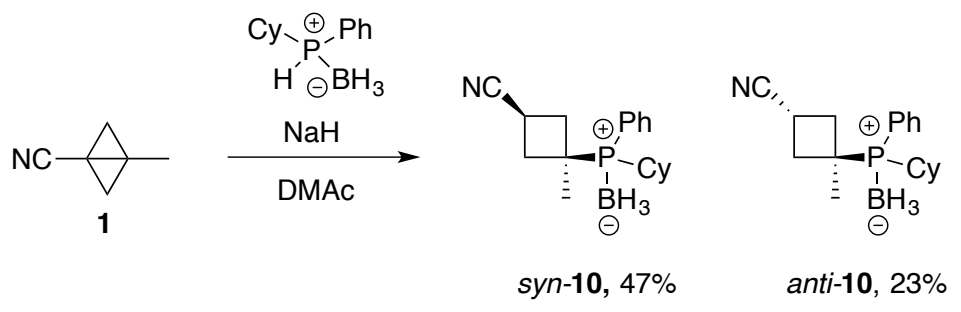

3-(Cyclohexyl(phenyl)phosphanyl)-3-methylcyclobutane-1-carbonitrile borane 10.

A solution of racemic phenyl cyclohexyl phosphine borane ${ }^{4}(0.275 \mathrm{~g}, 1.33 \mathrm{mmol})$ and bicyclobutane 1 (0.136 g, $1.47 \mathrm{mmol}, 1.20 \mathrm{eq})$ in DMAc $(8 \mathrm{~mL})$ was sparged with Ar for $10 \mathrm{~min}$ in a $25 \mathrm{~mL}$ flask, cooled on an ice bath, and treated with $\mathrm{NaH}(0.059 \mathrm{~g}, 1.5 \mathrm{mmol}, 1.2 \mathrm{eq}, 60 \%$ in oil) in one portion, affording a yellow solution that gradually faded to brown. The ice bath was removed, and the solution was warmed to $\mathrm{rt}$ for $1 \mathrm{~h}$. The orange/brown reaction mixture was quenched with sat. aq. $\mathrm{NH}_{4} \mathrm{Cl}(50 \mathrm{~mL})$, and partitioned between water $(100 \mathrm{~mL})$ and ether $(100$ $\mathrm{mL})$. The aqueous layer was extracted with ether $(70 \mathrm{~mL})$. The combined organic layers were washed with 1:1 water/brine $(100 \mathrm{~mL})$, dried $\left(\mathrm{Na}_{2} \mathrm{SO}_{4}\right)$ and concentrated. Chromatography on $\mathrm{SiO}_{2}$ (15-40\% EtOAc/hexane) afforded syn-10 (0.189 g, $\left.0.632 \mathrm{mmol}, 47 \%\right)$ as a colorless solid and anti-10 (0.092 g, $0.31 \mathrm{mmol}, 23 \%)$ as a colorless oil. Syn-10: Mp 137-139 ${ }^{\circ} \mathrm{C} ;{ }^{1} \mathrm{H}$ NMR (400 MHz, $\left.\mathrm{CDCl}_{3}\right) \delta 7.68(\mathrm{td}, 2 \mathrm{H}, J=8.4,1.6 \mathrm{~Hz}$ ), 7.55-7.42 (m, $3 \mathrm{H}$ ), 3.30 (pent, $1 \mathrm{H}, J=9.2 \mathrm{~Hz}$ ), 3.13 (ddd, 1 $\mathrm{H}, J=12.8 \mathrm{~Hz}, 10.0 \mathrm{~Hz}, 7.6 \mathrm{~Hz}$ ), 2.90 (ddd, $1 \mathrm{H}, J=13.6 \mathrm{~Hz}, 11.6 \mathrm{~Hz}, 9.6 \mathrm{~Hz}), 2.41-2.28(\mathrm{~m}, 1 \mathrm{H})$, 2.10 (ddd, $1 \mathrm{H}, J=16.0 \mathrm{~Hz}, 4.4 \mathrm{~Hz}, 3.2 \mathrm{~Hz}$ ), 1.93-1.83 (m, $1 \mathrm{H}), 1.79-1.55(\mathrm{~m}, 5 \mathrm{H}), 1.51-1.18$ (m, 5 $\mathrm{H}), 1.42\left(\mathrm{~d}, 3 \mathrm{H},{ }^{3} J_{\mathrm{PH}}=11.2 \mathrm{~Hz}\right), 1.1-0.3(\mathrm{br} \mathrm{m}, 3 \mathrm{H}) ;{ }^{13} \mathrm{C} \mathrm{NMR}\left(100 \mathrm{MHz}, \mathrm{CDCl}_{3}\right) \delta 132.9\left(\mathrm{~J}_{\mathrm{CP}}=7 \mathrm{~Hz}\right)$, $131.5\left(J_{\mathrm{CP}}=3 \mathrm{~Hz}\right), 128.8,128.7,125.0,124.5,120.7,34.8,34.2,32.2\left({ }^{1} J_{\mathrm{CP}}=30 \mathrm{~Hz}\right), 30.3\left({ }^{1} J_{\mathrm{CP}}=33\right.$ $\mathrm{Hz}), 26.6,26.5,26.4,26.3,25.7,20.9,18.3\left({ }^{2} J_{\mathrm{CP}}=18 \mathrm{~Hz}\right) ;{ }^{31} \mathrm{P} \mathrm{NMR}\left(162 \mathrm{MHz}, \mathrm{CDCl}_{3}\right) \delta$ 32.0; IR (ATR) 2934, 2397, 1448, 1072, $751 \mathrm{~cm}^{-1}$; MS $\left(\mathrm{ESI}^{+}\right) \mathrm{m} / \mathrm{z}$ 300, 286, 231, 209, 173, 150; HRMS (ESI ${ }^{+}$) $\mathrm{m} / z$ calcd for $\mathrm{C}_{18} \mathrm{H}_{28} \mathrm{BNP}[\mathrm{M}+\mathrm{H}], 300.2047$, found 300.2060. Anti-10: ${ }^{1} \mathrm{H}$ NMR (400 MHz, $\left.\mathrm{CDCl}_{3}\right) \delta$ 7.70 (td, $2 \mathrm{H}, J=8.0 \mathrm{~Hz}, 1.2 \mathrm{~Hz}$ ), 7.54-7.41 (m, 3 H), 3.32 (dddd, $1 \mathrm{H}, J=9.6 \mathrm{~Hz}, 9.6 \mathrm{~Hz}, 7.2 \mathrm{~Hz}, 7.2$ Hz), 3.15 (app q, $1 \mathrm{H}, J=12.0 \mathrm{~Hz}$ ), $2.71(\mathrm{td}, 1 \mathrm{H}, J=13.2 \mathrm{~Hz}, 11.2 \mathrm{~Hz}), 2.42-2.29(\mathrm{~m}, 1 \mathrm{H}), 2.23$ 
(ddd, $1 \mathrm{H}, J=12.4 \mathrm{~Hz}, 6.8 \mathrm{~Hz}, 6.4 \mathrm{~Hz}), 1.91-1.78(\mathrm{~m}, 2 \mathrm{H}), 1.77-1.62(\mathrm{~m}, 3 \mathrm{H}), 1.53\left(\mathrm{~d}, 3 \mathrm{H},{ }^{3} J_{\mathrm{PH}}=\right.$ 10.4 Hz), 1.49-1.38 (m, $1 \mathrm{H}), 1.38-1.15(\mathrm{~m}, 5 \mathrm{H}), 1.2-0.2(\mathrm{br} \mathrm{m}, 3 \mathrm{H}) ;{ }^{13} \mathrm{C} \mathrm{NMR}\left(100 \mathrm{MHz}, \mathrm{CDCl}_{3}\right) \delta$ $133.1\left(J_{\mathrm{CP}}=8 \mathrm{~Hz}\right), 131.7\left(J_{\mathrm{CP}}=2 \mathrm{~Hz}\right), 128.8,128.7,124.7,124.2,122.6,35.7\left(J_{\mathrm{CP}}=3 \mathrm{~Hz}\right), 34.9,32.3$ $\left({ }^{1} J_{\mathrm{CP}}=28 \mathrm{~Hz}\right), 30.6\left({ }^{1} J_{\mathrm{CP}}=33 \mathrm{~Hz}\right), 26.6\left(J_{\mathrm{CP}}=5 \mathrm{~Hz}\right), 26.5,26.4,26.3,26.2,25.6,24.0\left(J_{\mathrm{CP}}=3 \mathrm{~Hz}\right)$, $17.0\left(J_{\mathrm{CP}}=7 \mathrm{~Hz}\right) ;{ }^{31} \mathrm{P}$ NMR $\left(162 \mathrm{MHz}, \mathrm{CDCl}_{3}\right) \delta 30.2$; IR (ATR) 2939, 2858, 2384, 2239, 1437, 1109, $1066,742 \mathrm{~cm}^{-1}$

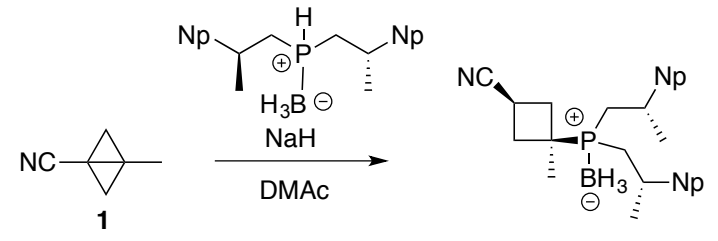

syn-11, 38\%

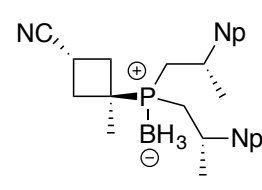

anti-11, 17\%

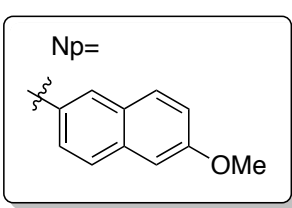

3-(Bis((S)-2-(6-methoxynaphthalen-2-yl)propyl)phosphanyl)-3-methylcyclobutane-1-carbonitrile borane 11.

A solution of di-(S)-naproxen phosphine borane $(3.77 \mathrm{~g}, 8.49 \mathrm{mmol})$ and bicyclobutane nitrile 1 $(0.790 \mathrm{~g}, 8.49 \mathrm{mmol})$ in DMAc $(40 \mathrm{~mL})$ was sparged with Ar for $10 \mathrm{~min}$ and cooled to $0^{\circ} \mathrm{C}$. NaH ( $0.340 \mathrm{~g}, 8.49 \mathrm{mmol}, 60 \%$ dispersion) was added in small portions, resulting in bubbling and the formation of an orange solution. The cooling bath was removed, and the reaction mixture was stirred at rt for $18 \mathrm{~h}$, and dropwise quenched with $5 \mathrm{M} \mathrm{NH}_{4} \mathrm{Cl}(10 \mathrm{~mL})$. The solution was partitioned between water $(40 \mathrm{~mL})$ and MTBE $(80 \mathrm{~mL})$. The aqueous layer was extracted with MTBE $(80 \mathrm{~mL})$, and the combined extracts were washed with water $(80 \mathrm{~mL})$, and brine $(80 \mathrm{~mL})$, dried $\left(\mathrm{MgSO}_{4}\right)$, filtered, and concentrated. The crude residue was purified by automated chromatography (120 $\mathrm{g} \mathrm{SiO}_{2}$ cartridge, 5-10-15-50\% EtOAc in hexane) to afford syn-11 (1.73 g, $3.22 \mathrm{mmol}, 38 \%)$ and anti-11 (0.773 g, $1.43 \mathrm{mmol}, 17 \%)$ as colorless solids. Syn-11: Mp 162-163 ${ }^{\circ} \mathrm{C} ;[\alpha]_{D}-24.1$ (c 0.220, $\mathrm{CH}_{2} \mathrm{Cl}_{2}$ ); ${ }^{1} \mathrm{H}$ NMR $\left(400 \mathrm{MHz}, \mathrm{CDCl}_{3}\right.$ ) $\delta 7.73$ (app t, $3 \mathrm{H}, J=9.2 \mathrm{~Hz}$ ), 7.52 (d, 2 $\mathrm{H}, J=8.4 \mathrm{~Hz}$ ), $7.36(\mathrm{dd}, 1 \mathrm{H}, J=8.4 \mathrm{~Hz}, 2.0 \mathrm{~Hz}), 7.22-7.16(\mathrm{~m}, 2 \mathrm{H}), 7.14(\mathrm{~d}, 1 \mathrm{H}, J=2.4 \mathrm{~Hz}), 7.09$ (dd, $1 \mathrm{H}, J=8.8 \mathrm{~Hz}, 2.4 \mathrm{~Hz}$ ), $7.02(\mathrm{~d}, 1 \mathrm{H}, J=2.4 \mathrm{~Hz}$ ), $6.92(\mathrm{dd}, 1 \mathrm{H}, J=8.4,1.6 \mathrm{~Hz}$ ), $3.95(\mathrm{~s}, 3 \mathrm{H})$, $3.89(\mathrm{~s}, 3 \mathrm{H}), 3.51-3.40(\mathrm{~m}, 1 \mathrm{H}), 3.10$ (pent, $1 \mathrm{H}, \mathrm{J}=9.2 \mathrm{~Hz}$ ), 2.97-2.86 (m, $1 \mathrm{H}), 2.79$ (app q, $1 \mathrm{H}, J$ $=12.0 \mathrm{~Hz}), 2.66(\mathrm{td}, 1 \mathrm{H}, J=13.2 \mathrm{~Hz}, 10.0 \mathrm{~Hz}), 1.98-1.87(\mathrm{~m}, 2 \mathrm{H}), 1.70-1.59(\mathrm{~m}, 2 \mathrm{H}), 1.46(\mathrm{~d}, 3 \mathrm{H}$, $J=7.2 \mathrm{~Hz}$ ), $1.21\left(\mathrm{~d}, 3 \mathrm{H},{ }^{3} J_{\mathrm{PH}}=11.0 \mathrm{~Hz}\right), 1.26-1.14(\mathrm{~m}, 1 \mathrm{H}), 1.08(\mathrm{ddd}, 1 \mathrm{H}, J=15.2 \mathrm{~Hz}, 9.2 \mathrm{~Hz}, 6.4$ 
$\mathrm{Hz}), 0.92(\mathrm{~d}, 3 \mathrm{H}, J=6.8 \mathrm{~Hz}), 1.0-0.3(\mathrm{br} \mathrm{m}, 3 \mathrm{H}) ;{ }^{13} \mathrm{C} \mathrm{NMR}\left(100 \mathrm{MHz}, \mathrm{CDCl}_{3}\right) \delta$ 157.7, 157.5, 141.5, $141.4,141.0,141.0,133.6,133.3,129.2$, 129.0, 129.0, 128.8, 127.5, 127.2, 126.0, 125.6, 125.5, 124.9, $120.6\left({ }^{4} J_{\mathrm{CP}}=2 \mathrm{~Hz}\right), 105.6,105.6,55.4,55.3,35.2,35.0,33.6,31.9\left({ }^{1} J_{\mathrm{CP}}=31 \mathrm{~Hz}\right), 30.0\left({ }^{1} J_{\mathrm{CP}}=\right.$ $31 \mathrm{~Hz}), 29.3\left({ }^{1} J_{\mathrm{CP}}=27 \mathrm{~Hz}\right), 25.6,25.0,20.5,17.8\left({ }^{2} J_{\mathrm{CP}}=18 \mathrm{~Hz}\right) ;{ }^{31} \mathrm{P} \mathrm{NMR}\left(162 \mathrm{MHz}, \mathrm{CDCl}_{3}\right) \delta$ 28.7; IR (ATR) 2933, 2386, 1603, 1450, 1215, $837 \mathrm{~cm}^{-1}$; MS (ESI ${ }^{+}$) m/z 538, 500, 447, 338; HRMS (ESI ${ }^{+}$) $\mathrm{m} / \mathrm{z}$ calcd for $\mathrm{C}_{34} \mathrm{H}_{42} \mathrm{BNO}_{2} \mathrm{P}[\mathrm{M}+\mathrm{H}]^{+}$538.3041, found 538.3025. Anti-11: $[\alpha]_{\mathrm{D}}+27.1$ (c 0.240, $\left.\mathrm{CH}_{2} \mathrm{Cl}_{2}\right) ;{ }^{1} \mathrm{H}$ NMR $\left(400 \mathrm{MHz}, \mathrm{CDCl}_{3}\right) \delta$ 7.78-7.72 (m, $\left.3 \mathrm{H}\right), 7.53(\mathrm{dd}, 2 \mathrm{H}, J=8.4 \mathrm{~Hz}, 6.0 \mathrm{~Hz}$ ), 7.38 (dd, $1 \mathrm{H}, J=8.4 \mathrm{~Hz}, 1.6 \mathrm{~Hz}$ ), 7.24-7.15 (m, $3 \mathrm{H}), 7.11(\mathrm{dd}, 1 \mathrm{H}, J=8.8 \mathrm{~Hz}, 2.4 \mathrm{~Hz}$ ), $7.05(\mathrm{~d}, 1 \mathrm{H}, J=$ $2.4 \mathrm{~Hz}$ ), 6.89 (dd, $1 \mathrm{H}, J=8.4,2.4 \mathrm{~Hz}), 3.96(\mathrm{~s}, 3 \mathrm{H}), 3.90(\mathrm{~s}, 3 \mathrm{H}), 3.53-3.43(\mathrm{~m}, 1 \mathrm{H}), 3.21-3.13(\mathrm{~m}$, $1 \mathrm{H}), 2.94-2.80(\mathrm{~m}, 2 \mathrm{H}), 2.49$ (dd, $1 \mathrm{H}, J=13.2 \mathrm{~Hz}, 10.4 \mathrm{~Hz}$ ), 2.11 (td, $1 \mathrm{H}, J=12.4 \mathrm{~Hz}, 7.2 \mathrm{~Hz}$ ), $1.94(\mathrm{td}, 1 \mathrm{H}, J=14.0 \mathrm{~Hz}, 9.6 \mathrm{~Hz}), 1.70-1.55(\mathrm{~m}, 3 \mathrm{H}), 1.47(\mathrm{~d}, 3 \mathrm{H}, J=6.4 \mathrm{~Hz}), 1.35\left(\mathrm{~d}, 3 \mathrm{H},{ }^{3} J_{\mathrm{PH}}=\right.$ 10.4 Hz), 1.13-1.04 (m, $1 \mathrm{H}), 0.93(\mathrm{~d}, 3 \mathrm{H}, J=7.2 \mathrm{~Hz}), 1.0-0.3(\mathrm{br} \mathrm{m}, 3 \mathrm{H}) ;{ }^{13} \mathrm{C}$ NMR $(100 \mathrm{MHz}$, $\left.\mathrm{CDCl}_{3}\right) \delta 157.7,157.5,141.6,141.5,140.9,140.9,133.7,133.3,129.2,129.0,128.8,127.6,127.2$, 126.1, 125.6, 125.3, 124.8, 122.5, 119.3, 105.7, 105.6, 55.4, 55.3, 35.2, 35.0, 34.7, 34.3, 34.2, 34.2, $32.1\left({ }^{1} J_{\mathrm{CP}}=31 \mathrm{~Hz}\right), 30.4\left({ }^{1} J_{\mathrm{CP}}=32 \mathrm{~Hz}\right), 29.6\left({ }^{1} J_{\mathrm{CP}}=27 \mathrm{~Hz}\right), 25.7\left(J_{\mathrm{CP}}=11 \mathrm{~Hz}\right), 24.9\left(J_{\mathrm{CP}}=7 \mathrm{~Hz}\right)$, $23.6\left(J_{C P}=3 \mathrm{~Hz}\right), 16.5\left({ }^{2} J_{C P}=7 \mathrm{~Hz}\right) ;{ }^{31} \mathrm{P} N M R\left(162 \mathrm{MHz}, \mathrm{CDCl}_{3}\right) \delta$ 27.5; IR (ATR) 2933, 2395, 1605, $1028,857 \mathrm{~cm}^{-1}$

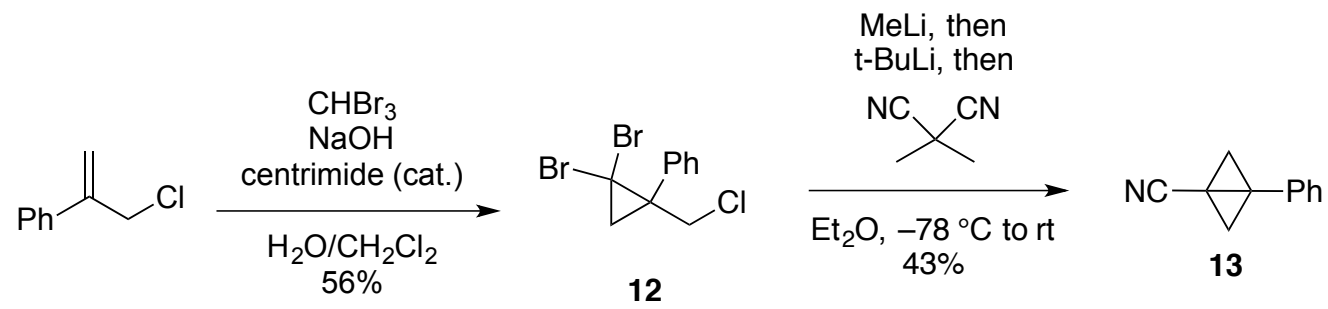

3-Phenylbicyclo[1.1.0]butane-1-carbonitrile 13. ${ }^{1}$

2-Phenyl allyl chloride $(26.1 \mathrm{~g}, 0.171 \mathrm{mmol})$, centrimide $(50 \mathrm{mg}$ ) and bromoform $(49 \mathrm{~mL}, 0.51$ mol) were combined in a 500-mL 4-necked flask equipped with a thermocouple, mechanical stirrer, Ar inlet, and addition funnel. The solution was cooled in an ice bath. The addition funnel was charged with a $50 \% \mathrm{NaOH}$ solution $(200 \mathrm{~mL}, 2.00 \mathrm{~mol})$, which was added dropwise over $1 \mathrm{~h}$. An exotherm to $7{ }^{\circ} \mathrm{C}$ was observed. he cooling bath was removed, and the reaction mixture turned brown and viscuous. After $2 \mathrm{~h}$, the solution had warmed up to $39{ }^{\circ} \mathrm{C}$ and became less viscuous, and a water bath was added to dissipate heat. The reaction mixture was then stirred 
for $20 \mathrm{~h}$ at rt, after which point the slurry was diluted with water $(200 \mathrm{~mL})$ and $\mathrm{CH}_{2} \mathrm{Cl}_{2}(300 \mathrm{~mL})$. The layers were separated, and the aqueous layer was re-extracted with $\mathrm{CH}_{2} \mathrm{Cl}_{2}(300 \mathrm{~mL})$. The combined extracts were washed with water $(2 \times 300 \mathrm{~mL})$, brine $(300 \mathrm{~mL})$, dried $\left(\mathrm{MgSO}_{4}\right)$, filtered, and concentrated. The crude residue was filtered through a 4" pad of $\mathrm{SiO}_{2}$ in a $600 \mathrm{~mL}$ frit using hexane $(200 \mathrm{~mL})$ followed by $15 \%$ EtOAc in hexane $(500 \mathrm{~mL})$. The eluent was concentrated to afford $51.7 \mathrm{~g}$ of crude product. Residual bromoform was removed by heating to ca. $50{ }^{\circ} \mathrm{C}$ under vacuum (2 Torr) to afford $12(31.0 \mathrm{~g}, 95.7 \mathrm{mmol}, 56 \%)$ as an amber oil that was used for the next step without further purification. A solution of dibromocyclopropane 12 $(8.28 \mathrm{~g}, 25.5 \mathrm{mmol})$ in diethyl ether $(60 \mathrm{~mL})$ was cooled to $-78{ }^{\circ} \mathrm{C}$ under $\mathrm{N}_{2}$ in a $250-\mathrm{mL}$ round bottom flask. Methyl lithium (15.6 mL, $25.5 \mathrm{mmol}, 1.63 \mathrm{M}$ in $\mathrm{Et}_{2} \mathrm{O}$ ) was added dropwise via syringe over $15 \mathrm{~min}$. After stirring for $1 \mathrm{~h}$ at $-78^{\circ} \mathrm{C}$, $t$-butyl lithium $(15.0 \mathrm{~mL}, 25.5 \mathrm{mmol}, 1.70 \mathrm{M}$ in pentane) was added dropwise via syringe over $15 \mathrm{~min}$. After stirring for an additional $1 \mathrm{~h}$ at $78{ }^{\circ} \mathrm{C}$, dimethyl malononitrile $(2.40 \mathrm{~g}, 25.5 \mathrm{mmol})$ was added as a solution in THF $(10 \mathrm{~mL})$, and the reaction mixture turned from a clear orange into an opaque yellow solution. The cooling bath was removed and the solution was warmed to rt over $1.5 \mathrm{~h}$, affording an orange/brown suspension. The mixture was quenched with sat. aq. $\mathrm{NH}_{4} \mathrm{Cl}(20 \mathrm{~mL})$, and partitioned between water $(30 \mathrm{~mL})$ and diethyl ether $(50 \mathrm{~mL})$. The aqueous layer was extracted with ether $(50 \mathrm{~mL})$. The combined organic extracts were washed with water $(60 \mathrm{~mL})$ and brine $(60 \mathrm{~mL})$, dried $\left(\mathrm{Na}_{2} \mathrm{SO}_{4}\right)$ and concentrated. Chromatography of the crude black residue on $\mathrm{SiO}_{2}(5-20 \%$ EtOAc/hexane) afforded bicyclobutane $13(1.71 \mathrm{~g}, 11.0 \mathrm{mmol}, 43 \%)$ as a yellow oil: ${ }^{1} \mathrm{H}$ NMR (300 $\left.\mathrm{MHz}, \mathrm{CDCl}_{3}\right) \delta$ 7.45-7.29 (m, $\left.\left.5 \mathrm{H}\right), 2.71(\mathrm{~s}, 2 \mathrm{H}), 1.68(\mathrm{~s}, 2 \mathrm{H}) ;{ }^{13} \mathrm{C} \mathrm{NMR} \mathrm{(100} \mathrm{MHz}, \mathrm{CDCl}_{3}\right) \delta$ 131.8, $128.9,128.5,128.0,126.5,118.8,37.9,29.5,5.8$.

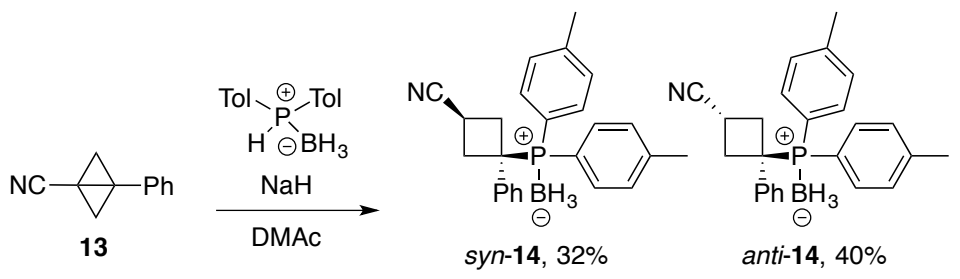

3-(Di-p-tolylphosphanyl)-3-phenylcyclobutane-1-carbonitrile borane 14.

A solution of nitrile $13(0.621 \mathrm{~g}, 4.01 \mathrm{mmol})$ and di-p-tolyl phosphine borane $(0.684 \mathrm{~g}, 3.00$ mmol) in DMAc (15 mL) was sparged with Ar for $10 \mathrm{~min}$, cooled to $0^{\circ} \mathrm{C}$, and treated with $\mathrm{NaH}$ $(0.120 \mathrm{~g}, 3.0 \mathrm{mmol})$ in one portion, resulting in bubbling. The cooling bath was removed. After 4 
$\mathrm{h}$ at $\mathrm{rt}$, the mixture was quenched with sat. aq. $\mathrm{NH}_{4} \mathrm{Cl}(10 \mathrm{~mL})$ and partitioned between MTBE and water $(50 \mathrm{~mL}$ each). The aqueous layer was extracted with MTBE $(30 \mathrm{~mL})$. The combined organic extracts were washed with water $(50 \mathrm{~mL})$, brine $(50 \mathrm{~mL})$, dried $\left(\mathrm{MgSO}_{4}\right)$, filtered, and concentrated to afford a crude residue that was purified by automated chromatography ( $80 \mathrm{~g}$ cartridge, 5-10-50\% EtOAc in hexane) to afford syn-14 (0.365 g, $0.95 \mathrm{mmol}, 32 \%)$ as a light yellow solid and anti-14 (0.457 g, $1.19 \mathrm{mmol}, 40 \%)$ as a white crystalline solid. Syn-14: Mp 68-72 ${ }^{\circ} \mathrm{C} ;{ }^{1} \mathrm{H}$ NMR $\left(400 \mathrm{MHz}, \mathrm{CDCl}_{3}\right) \delta 7.48(\mathrm{~d}, 2 \mathrm{H}, J=8.4 \mathrm{~Hz}), 7.45(\mathrm{~d}, 2 \mathrm{H}, J=8.4 \mathrm{~Hz}), 7.24-7.13(\mathrm{~m}, 7$ H), 7.09-7.02 (m, $2 \mathrm{H}$ ), 3.30 (ddd, $2 \mathrm{H}, J=13.2 \mathrm{~Hz}, 10.0 \mathrm{~Hz}, 2.4 \mathrm{~Hz}$ ), 3.03 (pent, $1 \mathrm{H}, J=9.2 \mathrm{~Hz}$ ), 2.91-2.84 (m, $2 \mathrm{H}), 2.38(\mathrm{~s}, 6 \mathrm{H}), 1.5-0.5(\mathrm{br} \mathrm{m}, 3 \mathrm{H}) ;{ }^{13} \mathrm{C} \mathrm{NMR}\left(100 \mathrm{MHz}, \mathrm{CDCl}_{3}\right) \delta 142.2\left(\mathrm{~J}_{\mathrm{CP}}=2\right.$ $\mathrm{Hz}), 137.6,133.9\left(J_{\mathrm{CP}}=6 \mathrm{~Hz}\right), 129.4\left(J_{\mathrm{CP}}=11 \mathrm{~Hz}\right), 128.9\left(J_{\mathrm{CP}}=3 \mathrm{~Hz}\right), 127.8,127.8,127.7,127.5$, 127.4, 122.6, 122.1, $120.2\left({ }^{4} J_{C P}=3 \mathrm{~Hz}\right), 43.7\left({ }^{1} J_{\mathrm{CP}}=27 \mathrm{~Hz}\right), 35.8,21.5,19.1\left(J_{\mathrm{CP}}=19 \mathrm{~Hz}\right) ;{ }^{31} \mathrm{P} N M R$ $\left(202 \mathrm{MHz}, \mathrm{CDCl}_{3}\right.$ ) $\delta$ 29.0; IR (ATR) 2368, 1600, 1497, 1446, 1190, 1063, 806, $655 \mathrm{~cm}^{-1}$; Anti-14: Mp 172-174 ${ }^{\circ} \mathrm{C}$; IR (ATR) 2933, 2391, 1604, 1450, $1064 \mathrm{~cm}^{-1} ;{ }^{1} \mathrm{H}$ NMR (400 MHz, CDCl $) \delta 7.44$ (d, $2 \mathrm{H}, J=8.4 \mathrm{~Hz}$ ), $7.42(\mathrm{~d}, 2 \mathrm{H}, J=8.0 \mathrm{~Hz}), 7.25-7.13(\mathrm{~m}, 7 \mathrm{H}), 6.66(\mathrm{dd}, 2 \mathrm{H}, J=6.4 \mathrm{~Hz}, 1.6 \mathrm{~Hz}), 3.55$ (pent, $1 \mathrm{H}, J=9.2 \mathrm{~Hz}$ ), 3.45-3.34 (m, $2 \mathrm{H}), 2.96-2.87(\mathrm{~m}, 2 \mathrm{H}), 2.38(\mathrm{~s}, 6 \mathrm{H}), 1.3-0.7(\mathrm{br} \mathrm{m}, 3 \mathrm{H}) ;{ }^{13} \mathrm{C}$ $\operatorname{NMR}\left(100 \mathrm{MHz}, \mathrm{CDCl}_{3}\right) \delta 142.3\left(J_{\mathrm{CP}}=3 \mathrm{~Hz}\right), 142.2\left(J_{\mathrm{CP}}=5 \mathrm{~Hz}\right), 134.2\left(J_{\mathrm{CP}}=98 \mathrm{~Hz}\right), 129.2,129.1$, $128.0\left(J_{\mathrm{CP}}=2 \mathrm{~Hz}\right), 127.6\left(J_{\mathrm{CP}}=2 \mathrm{~Hz}\right), 127.1,122.1,121.7,121.5,42.9\left({ }^{1} J_{\mathrm{CP}}=24 \mathrm{~Hz}\right), 36.7,21.5$, $17.7 ;{ }^{31} \mathrm{P} N M R\left(162 \mathrm{MHz}, \mathrm{CDCl}_{3}\right) \delta 25.7$; IR (ATR) 2933, 2383, 1437, 1065, 757, $744 \mathrm{~cm}^{-1}$; MS (ESI ${ }^{+}$) $m / z$ 384, 370, 345, 155; HRMS (ESI ${ }^{+}$) $m / z$ calcd for $\mathrm{C}_{25} \mathrm{H}_{28} \mathrm{BNP}[\mathrm{M}+\mathrm{H}] 384.2047$, found 384.2038.

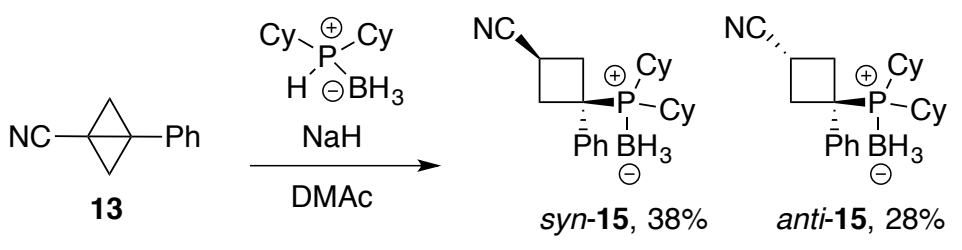

3-(Dicyclohexylphosphanyl)-3-phenylcyclobutane-1-carbonitrile borane 15.

A solution of nitrile $13(0.083 \mathrm{~g}, 0.54 \mathrm{mmol}, 1.3 \mathrm{eq})$ and dicyclohexyl phosphine borane $(0.087 \mathrm{~g}$, $0.41 \mathrm{mmol})$ in DMAc $(2 \mathrm{~mL})$ was sparged with Ar for $10 \mathrm{~min}$, cooled to $0{ }^{\circ} \mathrm{C}$, and trated with $\mathrm{NaH}$ $(0.016 \mathrm{~g}, 0.41 \mathrm{mmol}, 60 \%$ in oil) in one portion. The reaction mixture was stirred for $10 \mathrm{~min}$ in an ice bath, then warmed to rt over $1 \mathrm{~h}$ and quenched with sat aq $\mathrm{NH}_{4} \mathrm{Cl}(2 \mathrm{~mL})$. The solution was partitioned between water $(20 \mathrm{~mL})$ and diethyl ether $(20 \mathrm{~mL})$, and the aqueous layer was extracted with ether $(20 \mathrm{~mL})$. The combined organic extracts were washed with water $(40 \mathrm{~mL})$ 
and brine $(40 \mathrm{~mL})$, dried $\left(\mathrm{Na}_{2} \mathrm{SO}_{4}\right)$ and concentrated. The residue was purified by chromatography on $\mathrm{SiO}_{2}$ (15\% EtOAc/hexane) to afford the less polar anti-15 (0.043 g, 0.117 mmol, 28\%) and the more polar syn-15 (0.058 g, $0.158 \mathrm{mmol}, 38 \%)$, both as colorless solids. Syn-

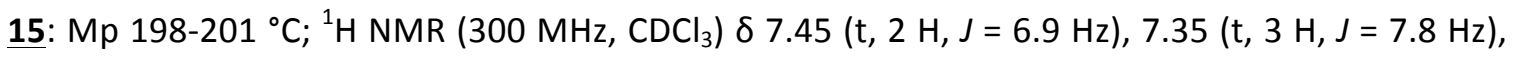
3.64-3.48 (m, $2 \mathrm{H}), 3.07$ (pent, $1 \mathrm{H}, J=9.2 \mathrm{~Hz}$ ), 2.76 (tt, $2 \mathrm{H}, J=8.7 \mathrm{~Hz}, 3.0 \mathrm{~Hz}$ ), 1.95-1.53 (m, 11 $\mathrm{H}), 1.32-1.05(\mathrm{~m}, 11 \mathrm{H}), 1.1-0.1(\mathrm{br} \mathrm{m}, 3 \mathrm{H}) ;{ }^{13} \mathrm{C} \mathrm{NMR}\left(100 \mathrm{MHz}, \mathrm{CDCl}_{3}\right) \delta 139.1\left(\mathrm{~J}_{\mathrm{CP}}=5 \mathrm{~Hz}\right), 128.9$, 128.5, $127.8\left(J_{\mathrm{CP}}=2 \mathrm{~Hz}\right), 127.6,127.6,124.5,120.2\left({ }^{4} J_{\mathrm{CP}}=3 \mathrm{~Hz}\right), 42.1\left({ }^{1} J_{\mathrm{CP}}=21 \mathrm{~Hz}\right), 36.9,36.6$, $31.5\left({ }^{1} J_{\mathrm{CP}}=28 \mathrm{~Hz}\right), 27.4,27.2,27.1,27.0,26.9,26.8,25.9,20.6\left({ }^{2} J_{\mathrm{CP}}=18 \mathrm{~Hz}\right) ;{ }^{31} \mathrm{P} \mathrm{NMR}(162 \mathrm{MHz}$, $\mathrm{CDCl}_{3}$ ) $\delta$ 39.6; IR (ATR) 2934, 2844, 2391, 1448, $1072 \mathrm{~cm}^{-1}$; MS $\left(\mathrm{ESI}^{+}\right) \mathrm{m} / \mathrm{z} 385\left(\mathrm{M}+\mathrm{NH}_{4}{ }^{+}\right), 368$, 273, 215; HRMS $\left(\mathrm{ESI}^{+}\right) \mathrm{m} / \mathrm{z}$ calcd for $\mathrm{C}_{23} \mathrm{H}_{36} \mathrm{BNP}[\mathrm{M}+\mathrm{H}]^{+}$368.2673, found 368.2696. Anti-15: Mp 205-207 ${ }^{\circ} \mathrm{C} ;{ }^{1} \mathrm{H}$ NMR $\left(400 \mathrm{MHz}, \mathrm{CDCl}_{3}\right) \delta 7.41$ (t, $2 \mathrm{H}, J=7.2 \mathrm{~Hz}$ ), 7.33 (app t, $1 \mathrm{H}, J=7.6 \mathrm{~Hz}$ ), 7.13 (d, $2 \mathrm{H}, J=8.0 \mathrm{~Hz}$ ), 3.67 (pent, $1 \mathrm{H}, J=9.2 \mathrm{~Hz}$ ), 3.38 (td, $2 \mathrm{H}, J=13.2 \mathrm{~Hz}, 10.0 \mathrm{~Hz}), 2.98-2.86(\mathrm{~m}, 2$ $\mathrm{H}), 1.95-1.49(\mathrm{~m}, 10 \mathrm{H}), 1.47-1.32(\mathrm{~m}, 2 \mathrm{H}), 1.32-1.02(\mathrm{~m}, 10 \mathrm{H}), 0.9-0.0(\mathrm{br} \mathrm{m}, 3 \mathrm{H}) ;{ }^{13} \mathrm{C} \mathrm{NMR}(100$ $\left.\mathrm{MHz}, \mathrm{CDCl}_{3}\right) \delta 143.0\left({ }^{2} J_{\mathrm{CP}}=6 \mathrm{~Hz}\right), 128.8,127.4,126.3\left({ }^{3} \mathrm{~J}_{\mathrm{CP}}=3 \mathrm{~Hz}\right), 121.8,41.4\left({ }^{1} J_{\mathrm{CP}}=21 \mathrm{~Hz}\right), 37.3$, $31.3\left({ }^{1} J_{\mathrm{CP}}=29 \mathrm{~Hz}\right), 28.6,27.6,27.2,27.1,26.7,26.6,25.8,17.6\left({ }^{2} J_{\mathrm{CP}}=3 \mathrm{~Hz}\right) ;{ }^{31} \mathrm{P} N M R(162 \mathrm{MHz}$, $\mathrm{CDCl}_{3}$ ) $\delta$ 35.9; IR (ATR) 2930, 2856, 2377, 2239, 1446, 1064, $760 \mathrm{~cm}^{-1}$.

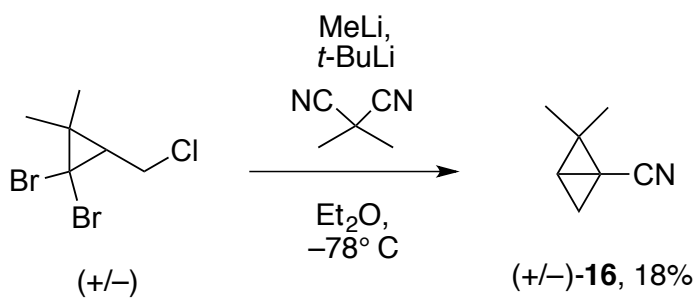

2,2-Dimethylbicyclo[1.1.0]butane-1-carbonitrile $16 .^{5}$

A solution of dibromocyclopropane $(10.0 \mathrm{~g}, 18.1 \mathrm{mmol})$ in $\mathrm{Et}_{2} \mathrm{O}(80 \mathrm{~mL})$ under $\mathrm{N}_{2}$ was cooled to $78{ }^{\circ} \mathrm{C}$ (dry ice/acetone) in a 250-mL, 3 necked flask equipped with an $\mathrm{N}_{2}$ inlet, septum, and internal thermometer. The reaction mixture was treated with methyl lithium $(22.2 \mathrm{~mL}, 36.2$ mmol, $1.63 \mathrm{M}$ in $\mathrm{Et}_{2} \mathrm{O}$ ) via syringe over $10 \mathrm{~min}$. After stirring for $1 \mathrm{~h}$ at $-78^{\circ} \mathrm{C}$, tert-butyl lithium $(21.3 \mathrm{~mL}, 36.2 \mathrm{mmol}, 1.70 \mathrm{M}$ in pentane) was added dropwise via syringe over $10 \mathrm{~min}$. The resulting yellow solution was stirred at $-78{ }^{\circ} \mathrm{C}$ for $1 \mathrm{~h}$, then a solution of dimethylmalonitrile ( $4.43 \mathrm{~g}, 47.0 \mathrm{mmol}, 1.30 \mathrm{eq})$ in THF $(10 \mathrm{~mL})$ was added via syringe over $3 \mathrm{~min}$. The cooling bath was removed, and the solution was warmed to $\mathrm{rt}$ for $2 \mathrm{~h}$ (a red color developed). The mixture 
was quenched with sat. aq. $\mathrm{NH}_{4} \mathrm{Cl}(10 \mathrm{~mL})$, and partitioned between water $(50 \mathrm{~mL})$ and $\mathrm{Et}_{2} \mathrm{O}(50$ $\mathrm{mL})$. The aqueous layer was extracted with ether $(50 \mathrm{~mL})$. The combined organic extracts were washed with water $(75 \mathrm{~mL})$, and brine $(75 \mathrm{~mL})$, dried $\left(\mathrm{Na}_{2} \mathrm{SO}_{4}\right)$ and concentrated. The brown oily residue was purified by chromatography on $\mathrm{SiO}_{2}$ (5-20\% EtOAc in hexanes) to afford $\mathbf{1 6}$ (0.694 g, $6.48 \mathrm{mmol}, 18 \%)$ as a colorless oil: ${ }^{1} \mathrm{H}$ NMR $\left(300 \mathrm{MHz}, \mathrm{CDCl}_{3}\right) \delta 2.30(\mathrm{dd}, 1 \mathrm{H}, J=3.0 \mathrm{~Hz}, 3.0 \mathrm{~Hz}$ ), $2.21(\mathrm{t}, 1 \mathrm{H}, J=3.0 \mathrm{~Hz}), 1.86(\mathrm{t}, 1 \mathrm{H}, J=3.0 \mathrm{~Hz}), 1.35(\mathrm{~s}, 3 \mathrm{H}), 0.96(\mathrm{~s}, 3 \mathrm{H}) ;{ }^{13} \mathrm{C} \mathrm{NMR}(100 \mathrm{MHz}$, $\left.\mathrm{CDCl}_{3}\right) \delta 120.6,53.8,35.3,24.9,22.0,13.8,2.6$.

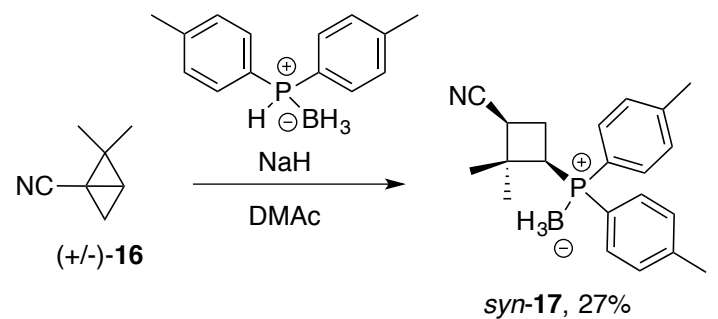

3-(Di-p-tolylphosphanyl)-2,2-dimethylcyclobutane-1-carbonitrile borane 17.

A solution of bicyclobutane $16(0.049 \mathrm{~g}, 0.46 \mathrm{mmol})$ and ditolylphosphine borane $(0.081 \mathrm{~g}, 0.36$ mmol) in DMAc $(2.5 \mathrm{~mL})$ was sparged with Ar for $10 \mathrm{~min}$, cooled to $0{ }^{\circ} \mathrm{C}$, and treated with $\mathrm{NaH}$ ( $0.018 \mathrm{~g}, 0.46 \mathrm{mmol}, 60 \%$ in mineral oil). The reaction mixture was warmed to $\mathrm{rt}$ for $1 \mathrm{~h}$, quenched with sat. aq. $\mathrm{NH}_{4} \mathrm{Cl}(3 \mathrm{~mL})$ and partitioned between water $(20 \mathrm{~mL})$ and $\mathrm{Et}_{2} \mathrm{O}(20 \mathrm{~mL})$. The aqueous layer was extracted with $\mathrm{Et}_{2} \mathrm{O}(20 \mathrm{~mL})$. The combined organic extracts were washed with water $(30 \mathrm{~mL})$, brine $(30 \mathrm{~mL})$, dried $\left(\mathrm{Na}_{2} \mathrm{SO}_{4}\right)$ and concentrated. Purification of the residue by chromatography on $\mathrm{SiO}_{2}$ (5-25\% EtOAc/hexane) afforded $0.032 \mathrm{~g}$ (0.095 mmol, 27\%) of syn-17 as a colorless oil: ${ }^{1} \mathrm{H}$ NMR $\left(400 \mathrm{MHz}, \mathrm{CDCl}_{3}\right) \delta 7.59(\mathrm{~d}, 1 \mathrm{H}, J=8.0 \mathrm{~Hz}), 7.56(\mathrm{~d}, 1 \mathrm{H}, \mathrm{J}=$ $8.4 \mathrm{~Hz}$ ), 7.45 (d, $1 \mathrm{H}, J=8.0 \mathrm{~Hz}$ ), $7.42(\mathrm{~d}, 1 \mathrm{H}, J=8.4 \mathrm{~Hz}$ ), 7.27 (dd, $2 \mathrm{H}, \mathrm{d}, 2 \mathrm{H}, J=8.0 \mathrm{~Hz}, 2.0 \mathrm{~Hz}$ ), $7.22(\mathrm{dd}, 2 \mathrm{H}, J=8.0 \mathrm{~Hz}, 1.6 \mathrm{~Hz}$ ), $3.04(\mathrm{ddd}, 1 \mathrm{H}, J=11.2 \mathrm{~Hz}, 8.4 \mathrm{~Hz}, 8.4 \mathrm{~Hz}), 2.93(\mathrm{td}, 1 \mathrm{H}, J=10.0$ $\mathrm{Hz}, 0.8 \mathrm{~Hz}$ ), 2.73 (pent, $1 \mathrm{H}, J=11.2 \mathrm{~Hz}$ ), 2.48 (ddd, $1 \mathrm{H}, J=19.2 \mathrm{~Hz}, 8.4 \mathrm{~Hz}, 2.4 \mathrm{~Hz}$ ), $2.39(\mathrm{~s}, 3 \mathrm{H})$, 2.37 (s, $3 \mathrm{H}), 1.27(\mathrm{~s}, 3 \mathrm{H}), 1.16(\mathrm{~s}, 3 \mathrm{H}), 1.2-0.6(\mathrm{br} \mathrm{m}, 3 \mathrm{H}) ;{ }^{13} \mathrm{C} \mathrm{NMR}\left(100 \mathrm{MHz}, \mathrm{CDCl}_{3}\right) \delta 141.9$ $\left({ }^{1} J_{\mathrm{CP}}=35 \mathrm{~Hz}\right), 141.9\left({ }^{1} J_{\mathrm{CP}}=35 \mathrm{~Hz}\right), 132.5\left({ }^{2} J_{\mathrm{CP}}=15 \mathrm{~Hz}\right), 132.4\left({ }^{2} J_{\mathrm{CP}}=14 \mathrm{~Hz}\right), 129.7,129.7,129.6$, 129.6, 126.3, 125.8, 125.3, 124.7, $118.5\left({ }^{4} J_{\mathrm{CP}}=6 \mathrm{~Hz}\right), 45.3,39.9\left({ }^{1} J_{\mathrm{CP}}=28 \mathrm{~Hz}\right), 33.8\left(J_{\mathrm{CP}}=25 \mathrm{~Hz}\right)$, 30.9, 24.9, 21.5, 21.5, $20.9\left({ }^{3} \mathrm{JP}_{\mathrm{CP}}=5 \mathrm{~Hz}\right) ;{ }^{31} \mathrm{P} \mathrm{NMR}\left(162 \mathrm{MHz}, \mathrm{CDCl}_{3}\right) \delta$ 13.7; IR (ATR) 2974, 2391, 2235, 1450, 1103, 1059, $734 \mathrm{~cm}^{-1}$; MS $\left.\left(\mathrm{ESI}^{+}\right) \mathrm{m} / \mathrm{z} 336,322,282,249,228,209 ; \mathrm{HRMS}(\mathrm{ESI})^{+}\right) \mathrm{m} / \mathrm{z}$ 
calcd for $\mathrm{C}_{21} \mathrm{H}_{28} \mathrm{BNP}[\mathrm{M}+\mathrm{H}]^{+} 336.2047$, found 336.2061.

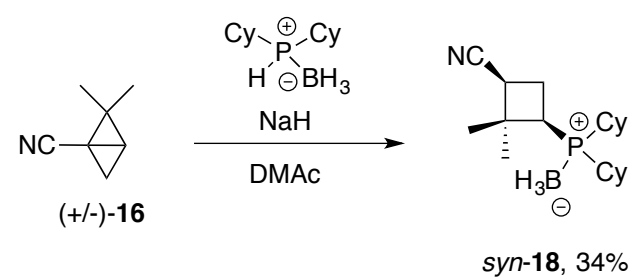

3-(Dicyclohexylphosphanyl)-2,2-dimethylcyclobutane-1-carbonitrile borane 18.

A solution of bicyclobutane 16 (0.148 g, $1.37 \mathrm{mmol})$ and dicyclohexyl phosphine borane $(0.225 \mathrm{~g}$, $1.06 \mathrm{mmol})$ in DMAc $(7.5 \mathrm{~mL})$ was sparged with Ar for $10 \mathrm{~min}$, cooled to $0{ }^{\circ} \mathrm{C}$ and treated with $\mathrm{NaH}(0.055 \mathrm{~g}, 1.37 \mathrm{mmol}, 60 \%$ in mineral oil). The reaction mixture was warmed to rt for $1.5 \mathrm{~h}$, quenched with sat. aq. $\mathrm{NH}_{4} \mathrm{Cl}(3 \mathrm{~mL})$, and partitioned between water $(30 \mathrm{~mL})$ and $\mathrm{Et}_{2} \mathrm{O}(30 \mathrm{~mL})$. The aqueous layer was extracted with $\mathrm{Et}_{2} \mathrm{O}(30 \mathrm{~mL})$. The combined organic extracts were washed with water $(50 \mathrm{~mL})$, and brine $(50 \mathrm{~mL})$, dried $\left(\mathrm{Na}_{2} \mathrm{SO}_{4}\right)$ and concentrated. Purification of the residue by chromatography on $\mathrm{SiO}_{2}$ (10-30\% EtOAc/hexane) afforded syn-18 (0.144 g, 0.339 mmol, 34\%) as a colorless solid: $\mathrm{Mp} 135-139{ }^{\circ} \mathrm{C} ;{ }^{1} \mathrm{H}$ NMR $\left(400 \mathrm{MHz}, \mathrm{CDCl}_{3}\right) \delta 2.90(\mathrm{app} \mathrm{t}, 1 \mathrm{H}, \mathrm{J}=$ $8.8 \mathrm{~Hz}$ ), 2.68 (pent, $1 \mathrm{H}, J=10.8 \mathrm{~Hz}$ ), 2.50 (td, $1 \mathrm{H}, J=10.8 \mathrm{~Hz}, 8.0 \mathrm{~Hz}$ ), 2.31 (dtd, $1 \mathrm{H}, J=10.4 \mathrm{~Hz}$, $8.0 \mathrm{~Hz}, 2.0 \mathrm{~Hz}), 2.05-1.96(\mathrm{~m}, 1 \mathrm{H}), 1.94-1.55(\mathrm{~m}, 1 \mathrm{H}), 1.51(\mathrm{~s}, 3 \mathrm{H}), 1.28(\mathrm{~s}, 3 \mathrm{H}), 1.37-1.14(\mathrm{~m}, 10$ H), 1.1-0.0 (br m, $3 \mathrm{H}) ;{ }^{13} \mathrm{C} \mathrm{NMR}\left(100 \mathrm{MHz}, \mathrm{CDCl}_{3}\right) \delta 118.4\left({ }^{4} \mathrm{~J}_{\mathrm{CP}}=5 \mathrm{~Hz}\right), 45.3\left({ }^{3} \mathrm{~J}_{\mathrm{CP}}=1 \mathrm{~Hz}\right), 36.3\left({ }^{1} \mathrm{~J}_{\mathrm{CP}}\right.$ $=25 \mathrm{~Hz}), 34.3\left({ }^{1} J_{\mathrm{CP}}=23 \mathrm{~Hz}\right), 33.6\left(J_{\mathrm{CP}}=10 \mathrm{~Hz}\right), 33.3\left(J_{\mathrm{CP}}=9 \mathrm{~Hz}\right), 31.2,27.8,27.5$ (two overlapping resonances), 27.4, 27.3, 27.1, 27.1, 27.0, 27.0, 26.9, 26.0, $25.9\left(J_{C P}=19 \mathrm{~Hz}\right), 25.4,21.9\left(J_{C P}=4\right.$ $\mathrm{Hz}) ;{ }^{31} \mathrm{P}$ NMR $\left(162 \mathrm{MHz}, \mathrm{CDCl}_{3}\right.$ ) $\delta$ 26.7; IR (ATR) 2924, 2365, 1448, $1066 \mathrm{~cm}^{-1} ; \mathrm{MS}\left(\mathrm{ESI}^{+}\right) \mathrm{m} / \mathrm{z} 320$, $306,268,241,215,273,152$; HRMS $\left(\mathrm{ESI}^{+}\right) \mathrm{m} / \mathrm{z}$ calcd for $\mathrm{C}_{19} \mathrm{H}_{36} \mathrm{BNP}[\mathrm{M}+\mathrm{H}]^{+} 320.2673$, found 320.2684 .

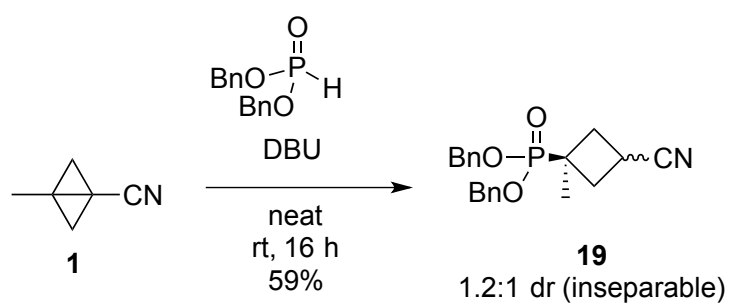

Dibenzyl (3-cyano-1-methylcyclobutyl)phosphonate 19.

A 2-mL vial was charged with bicyclobutane 1 (0.076 g, $0.82 \mathrm{mmol}), \mathrm{DBU}(0.12 \mathrm{~mL}, 0.82 \mathrm{mmol})$, 
and dibenzyl phosphite $(0.18 \mathrm{~mL}, 0.81 \mathrm{mmol})$. The colorless reaction mixture was stirred at $\mathrm{rt}$ under $\mathrm{N}_{2}$ for $16 \mathrm{~h}$, at which point a ${ }^{31} \mathrm{P}$ NMR analysis of an aliquot showed that the dibenzyl phosphite was consumed. The mixture was purified by chromatography on $\mathrm{SiO}_{2}(20-100 \%$ EtOAc/hexanes) to afford cyclobutane $19(0.172 \mathrm{~g}, 0.484 \mathrm{mmol}, 59 \%)$ as a colorless oily, inseparable 1.2:1 mixture of diastereomers: ${ }^{1} \mathrm{H}$ NMR $\left(400 \mathrm{MHz}, \mathrm{CDCl}_{3}\right) \delta$ 7.41-7.28 (m, $\left.20 \mathrm{H}\right)$, 5.11-4.88 (m, 8 H), 3.30-3.13 (m, 2 H), 2.89-2.71 (m, 4 H), 2.26-2.13 (m, 2 H), 2.11-2.01 (m, 2 H), $1.43\left(\mathrm{~d}, 3 \mathrm{H},{ }^{3} \mathrm{~J}_{\mathrm{PH}}=16.4 \mathrm{~Hz}\right), 1.41\left(\mathrm{~d}, 3 \mathrm{H},{ }^{3} \mathrm{~J}_{\mathrm{PH}}=17.2 \mathrm{~Hz}\right) ;{ }^{31} \mathrm{P} \mathrm{NMR}\left(162 \mathrm{MHz}, \mathrm{CDCl}_{3}\right) \delta 33.2$ (major), 30.5 (minor); IR (ATR) 2980, 2238, 1497, 1456, 1213, 966, 863, 826, $734 \mathrm{~cm}^{-1}$; HRMS $\left(\mathrm{ESI}^{+}\right) \mathrm{m} / \mathrm{z}$ calcd for $\mathrm{C}_{20} \mathrm{H}_{23} \mathrm{NO}_{3} \mathrm{P}[\mathrm{M}+\mathrm{H}] 356.1416$, found 356.1390 .

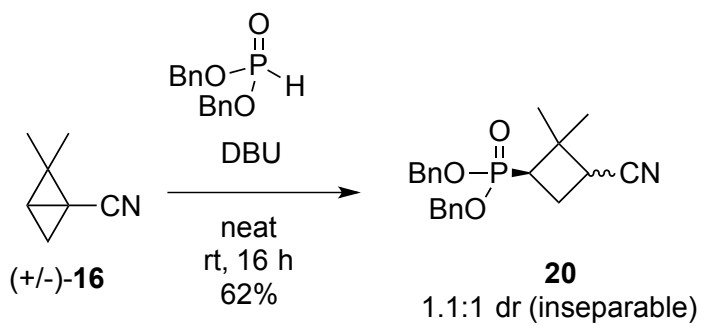

Dibenzyl (3-cyano-2,2-dimethylcyclobutyl)phosphonate.

A $10-\mathrm{mL}$ flask was charged with bicyclobutane $16(0.072 \mathrm{~g}, 0.67 \mathrm{mmol}), \mathrm{DBU}(0.10 \mathrm{~mL}, 0.67$ $\mathrm{mmol})$, and dibenzyl phosphite $(0.15 \mathrm{~mL}, 0.67 \mathrm{mmol})$. The neat, colorless mixture was stirred under $\mathrm{N}_{2}$ for $14 \mathrm{~h}$, at which point a ${ }^{31} \mathrm{P}$ NMR analysis of an aliquot showed that the dibenzyl phosphite was consumed. The mixture was purified by chromatography on $\mathrm{SiO}_{2}(5 \%$ $\left.\mathrm{MeOH} / \mathrm{CH}_{2} \mathrm{Cl}_{2}\right)$ to afford cyclobutane $20(0.153 \mathrm{~g}, 0.414 \mathrm{mmol}, 62 \%)$ as a colorless, viscous oil, inseparable 1.1:1 mixture of diastereomers: ${ }^{1} \mathrm{H}$ NMR $\left(400 \mathrm{MHz}, \mathrm{CDCl}_{3}\right) \delta 7.40-7.28(\mathrm{~m}, 20 \mathrm{H})$, 5.08-4.99 (m, 4 H), 4.97-4.85 (m, 4 H), 3.00 (ddd, 1 H, J = 9.2 Hz, 6.8 Hz, 2.4 Hz), 2.84-2.75 (m, 1 $\mathrm{H}), 2.51-2.17(\mathrm{~m}, 6 \mathrm{H}), 1.46(\mathrm{~s}, 3 \mathrm{H}), 1.34(\mathrm{~s}, 6 \mathrm{H}), 1.19(\mathrm{~s}, 3 \mathrm{H}) ;{ }^{31} \mathrm{P} \mathrm{NMR}\left(162 \mathrm{MHz}, \mathrm{CDCl}_{3}\right) \delta 29.5$ (minor), 26.7 (major).
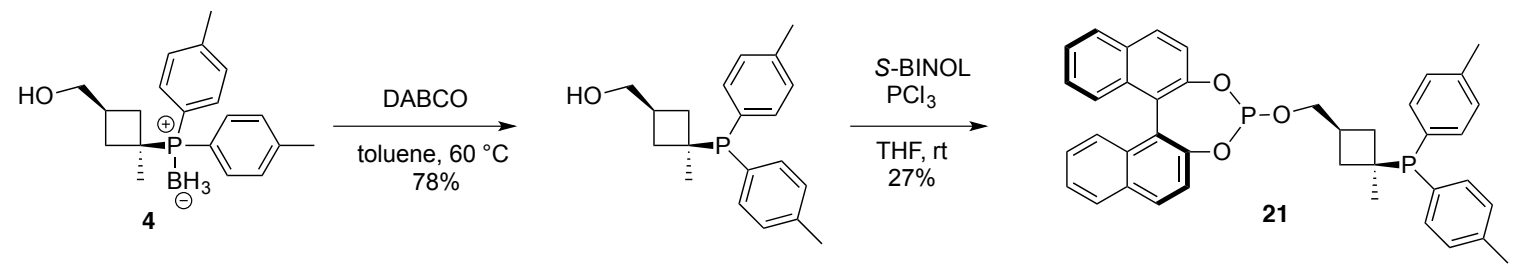

(11S)-4-((3-(Di-p-tolylphosphanyl)-3-methylcyclobutyl)methoxy)dinaphtho[2,1-d:1',2'- 


\section{$f[1,3,2]$ dioxaphosphepine 21.}

A solution of phosphine borane $4(0.160 \mathrm{~g}, 0.512 \mathrm{mmol})$ and DABCO $(0.230 \mathrm{~g}, 2.05 \mathrm{mmol})$ in toluene ( $2 \mathrm{~mL}$ ) was sparged with Ar for $5 \mathrm{~min}$, heated in the monowave microwave reactor at 60 ${ }^{\circ} \mathrm{C}$ for $1 \mathrm{~h}$, cooled, and partitioned between $0.5 \mathrm{M} \mathrm{HCl}(20 \mathrm{~mL})$ and $\mathrm{Et}_{2} \mathrm{O}(20 \mathrm{~mL})$. The aqueous layer was re-extracted with $\mathrm{Et}_{2} \mathrm{O}(25 \mathrm{~mL})$, and the combined organic layers were washed with brine $(30 \mathrm{~mL})$, dried $\left(\mathrm{Na}_{2} \mathrm{SO}_{4}\right)$ and concentrated to afford the phosphine $(0.119 \mathrm{~g}, 0.381 \mathrm{mmol}$, 78\%) as a colorless oil: ${ }^{1} \mathrm{H}$ NMR $\left(400 \mathrm{MHz}, \mathrm{CDCl}_{3}\right) \delta 7.28(\mathrm{~d}, 4 \mathrm{H}, J=7.2 \mathrm{~Hz}), 7.15(\mathrm{~d}, 4 \mathrm{H}, J=9.6$ Hz), $3.21(\mathrm{~d}, 2 \mathrm{H}, J=6.4 \mathrm{~Hz}$ ), 2.68 (pent, $1 \mathrm{H}, J=8.0 \mathrm{~Hz}$ ), 2.35 (s, $6 \mathrm{H}$ ), 2.07 (d, $1 \mathrm{H}, J=12.4 \mathrm{~Hz}$ ), $2.02(\mathrm{~d}, 1 \mathrm{H}, J=11.2 \mathrm{~Hz}), 2.00-1.91(\mathrm{~m}, 2 \mathrm{H}), 1.48\left(\mathrm{~d}, 3 \mathrm{H},{ }^{3} \mathrm{~J}_{\mathrm{PH}}=12.8 \mathrm{~Hz}\right) ;{ }^{31} \mathrm{P} N M R(162 \mathrm{MHz}$, $\left.\mathrm{CDCl}_{3}\right)$ 反 7.7. S-BINOL $(0.223 \mathrm{~g}, 0.778 \mathrm{mmol})$ was placed under $\mathrm{N}_{2}$ into a $50-\mathrm{mL}$ flask and treated with $\mathrm{PCl}_{3}(1.1 \mathrm{~mL}, 12 \mathrm{mmol}, 17 \mathrm{eq})$ and $\mathrm{N}$-methylpyrrolidone (1 drop). The mixture was stirred at $60{ }^{\circ} \mathrm{C}$ for $15 \mathrm{~min}$, cooled, and slowly concentrated under high vacuum to give a semi-solid. The residue was azeotroped with toluene $(5 \mathrm{~mL})$ and dissolved in THF $(5 \mathrm{~mL})$. An NMR of an aliquot showed the clean chlorophosphite $\left({ }^{31} \mathrm{P}\right.$ NMR $\left.\delta 174\right)$. In a separate $50-\mathrm{mL}$ flask, a solution of phosphine $(0.221 \mathrm{~g}, 0.707 \mathrm{mmol})$ in THF $(3 \mathrm{~mL})$ was cooled to $0{ }^{\circ} \mathrm{C}$ under $\mathrm{N}_{2}$. $\mathrm{NaH}(0.031 \mathrm{~g}, 0.79$ $\mathrm{mmol}, 1.1 \mathrm{eq}, 60 \%$ in oil) was added in one portion. The resulting alkoxide was stirred for $10 \mathrm{~min}$ at $0{ }^{\circ} \mathrm{C}$. The chlorophosphine solution was then transferred to the alkoxide solution via syringe at $0{ }^{\circ} \mathrm{C}$. The cooling bath was removed, and the reaction mixture was stirred for $19 \mathrm{~h}$ at rt, concentrated, and purified by chromatography on $\mathrm{SiO}_{2}$ (5-40\% EtOAc/hexane) to afford 21 (0.121 g, $0.193 \mathrm{mmol}, 27 \%)$ as a colorless oil: ${ }^{1} \mathrm{H} \mathrm{NMR}\left(400 \mathrm{MHz}, \mathrm{CDCl}_{3}\right) \delta 7.96(\mathrm{~d}, 1 \mathrm{H}, \mathrm{J}=8.8 \mathrm{~Hz})$, 7.95-7.86 (m, $3 \mathrm{H}), 7.47$ (d, $1 \mathrm{H}, J=8.8 \mathrm{~Hz}), 7.42(\mathrm{td}, 2 \mathrm{H}, J=6.8 \mathrm{~Hz}, 1.2 \mathrm{~Hz}), 7.37-7.22(\mathrm{~m}, 9 \mathrm{H})$, 7.18-7.12 (m, 4 H), 3.55 (app dd, $1 \mathrm{H},{ }^{3} \mathrm{JPH}_{\mathrm{PH}}=17.2 \mathrm{~Hz}, J=7.6 \mathrm{~Hz}$ ), 3.37 (app dd, $1 \mathrm{H},{ }^{3} \mathrm{~J}_{\mathrm{PH}}=17.6 \mathrm{~Hz}, J$ $=7.6 \mathrm{~Hz}$ ), 2.72 (pent, $1 \mathrm{H}, J=8.0 \mathrm{~Hz}$ ), $2.34(\mathrm{~s}, 6 \mathrm{H}$ ), 2.05 (dd, $2 \mathrm{H}, J=20 \mathrm{~Hz}, 12 \mathrm{~Hz}$ ), 1.96-1.88 (m, 2 H), $1.45\left(\mathrm{~d}, 3 \mathrm{H},{ }^{3} \mathrm{JPH}_{\mathrm{PH}}=12.8 \mathrm{~Hz}\right) ;{ }^{13} \mathrm{C} \mathrm{NMR}\left(100 \mathrm{MHz}, \mathrm{CDCl}_{3}\right) \delta 148.5\left(J_{\mathrm{CP}}=5 \mathrm{~Hz}\right), 147.5,138.5\left(J_{\mathrm{CP}}=\right.$ $5 \mathrm{~Hz}), 134.3\left(J_{\mathrm{CP}}=6 \mathrm{~Hz}\right), 134.1\left(J_{\mathrm{CP}}=6 \mathrm{~Hz}\right), 133.1,133.0,132.8,132.6,131.5,131.0,130.3,129.9$, $129.1\left(J_{\mathrm{CP}}=7 \mathrm{~Hz}\right), 128.3\left(J_{\mathrm{CP}}=6 \mathrm{~Hz}\right), 127.0,126.2\left(J_{\mathrm{CP}}=9 \mathrm{~Hz}\right), 125.0,124.9,124.1\left(J_{\mathrm{CP}}=5 \mathrm{~Hz}\right)$, $122.7\left(J_{C P}=2 \mathrm{~Hz}\right), 121.9,121.7,68.5\left({ }^{2} J_{\mathrm{CP}}=7 \mathrm{~Hz}\right), 34.7,34.7,34.6,34.6,31.8\left({ }^{1} J_{\mathrm{CP}}=18 \mathrm{~Hz}\right), 31.2$, $\left(J_{\mathrm{CP}}=10 \mathrm{~Hz}\right), 26.9,35.4,25.3\left(J_{\mathrm{CP}}=4 \mathrm{~Hz}\right), 21.4,20.7,14.2 ;{ }^{31} \mathrm{P} \mathrm{NMR}\left(162 \mathrm{MHz}, \mathrm{CDCl}_{3}\right) \delta$ 142.1, 7.8; HRMS $\left(E S I^{+}\right) \mathrm{m} / z$ calcd for $\mathrm{C}_{40} \mathrm{H}_{37} \mathrm{O}_{3} \mathrm{P}_{2}[\mathrm{M}+\mathrm{H}]^{+}$627.2212, found 627.2206. 


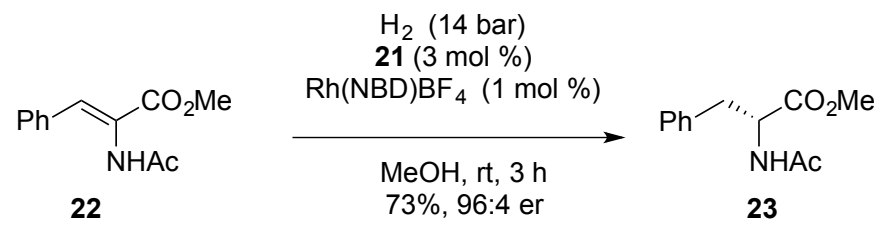

Methyl acetyl-D-phenylalaninate (23).

A solution of $22(0.116 \mathrm{~g}, 0.532 \mathrm{mmol})$ in $\mathrm{MeOH}(1 \mathrm{~mL})$ in a stainless steel pressure vessel was charged with a solution of phosphine-phosphite 21 (0.010 g, $0.016 \mathrm{mmol}, 3 \mathrm{~mol} \%)$ in $\mathrm{MeOH}(0.3$ $\mathrm{mL}$ ) and a solution of $\mathrm{Rh}(\mathrm{NBD}) \mathrm{BF}_{4}(0.002 \mathrm{~g}, 0.005 \mathrm{mmol}, 1 \mathrm{~mol} \%)$ in $\mathrm{MeOH}(0.3 \mathrm{~mL})$ under an $\mathrm{Ar}$ blanket provided by an inverted funnel. The resulting yellow solution was sealed in the Parr autoclave and placed under 13.99 bar of $\mathrm{H}_{2}$. After $3.5 \mathrm{~h}$, the remaining 13.96 bar was vented. The solution was concentrated, redissolved in $\mathrm{CH}_{2} \mathrm{Cl}_{2}(1 \mathrm{~mL})$, and filtered through neutral $\mathrm{Al}_{2} \mathrm{O}_{3}$ to afford 23 (0.086 g, $0.39 \mathrm{mmol}, 73 \%)$ as a colorless solid: ${ }^{1} \mathrm{H} \mathrm{NMR}\left(300 \mathrm{MHz}, \mathrm{CDCl}_{3}\right) \delta$ 7.38-7.21 (m $3 \mathrm{H}$ ), 7.16-7.07 (m, $2 \mathrm{H}$ ), 6.09 (d, $1 \mathrm{H}, J=6.6 \mathrm{~Hz}$ ), 4.90 (ddd, $1 \mathrm{H}, J=7.8 \mathrm{~Hz}, 6.0 \mathrm{~Hz}, 5.7 \mathrm{~Hz}$ ), $3.74(\mathrm{~s}, 3 \mathrm{H}), 3.21-3.04(\mathrm{~m}, 2 \mathrm{H}), 1.99$ (s, $3 \mathrm{H}$ ); Chiral SFC (Chiralpak IA, 5-15\% i-PrOH modifier gradient) $T_{r} 8.48 \mathrm{~min}$ (major, $D$-isomer), $11.42 \mathrm{~min}$ (minor, L-isomer). 


\section{DSC plot of nitrile 1}

DSC plot

August, 2015

DSC plot of Lot \# 103634-030 (liquid):

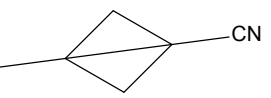

3-methylbicyclo[1.1.0]butane-1-carbonitrile

$\mathrm{C}_{6} \mathrm{H}_{7} \mathrm{~N}$

MW: 93.13

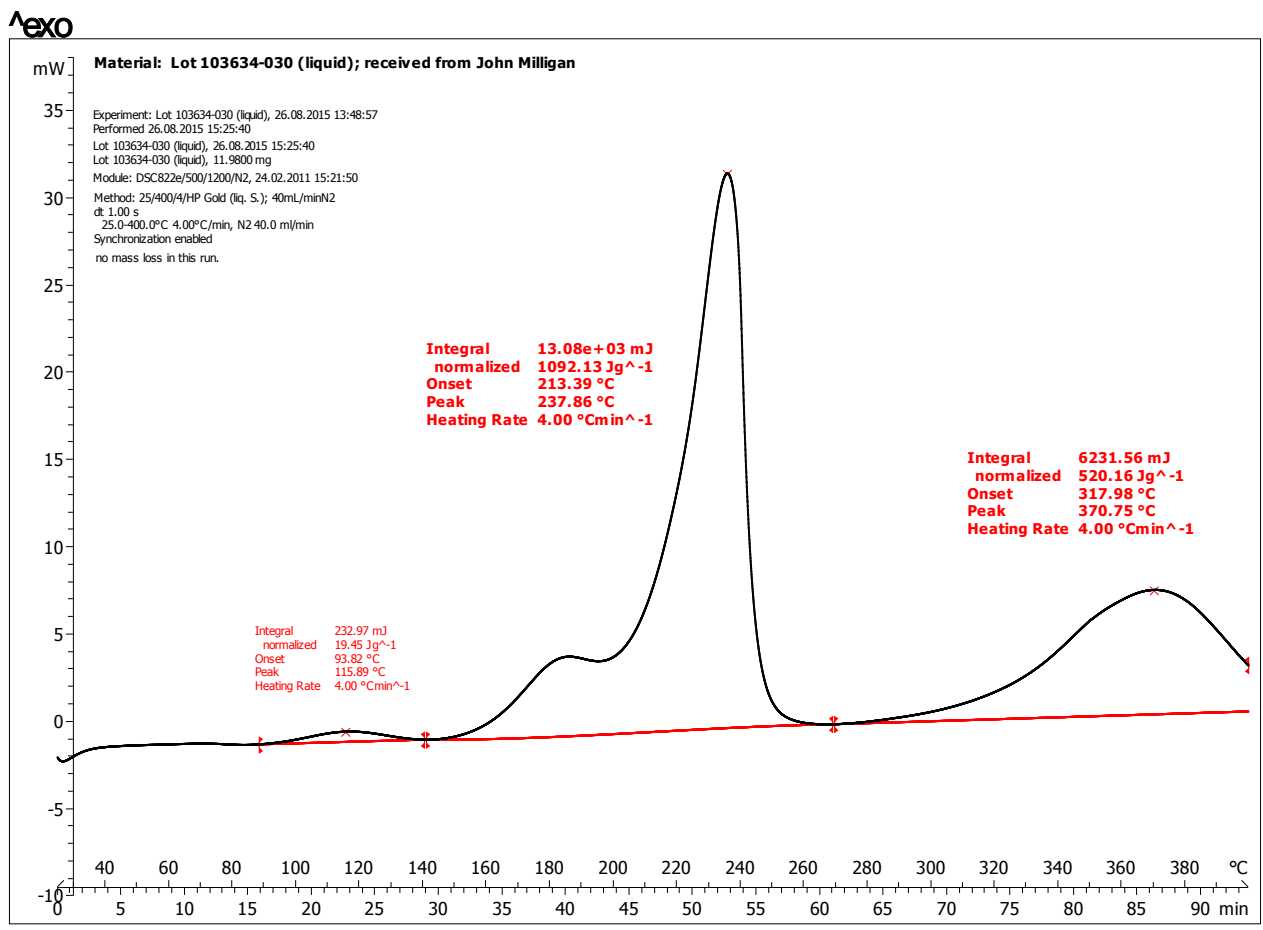

Lab: METTER

STAR ${ }^{\oplus}$ SW9.2

Method: $25^{\circ} \mathrm{C}$ to $400{ }^{\circ} \mathrm{C}$ with $4{ }^{\circ} \mathrm{C} /$ min heating rate, in a closed gold plated crucible.

Module: Mettler Toledo DSC 822e; sensor HSS8.

Sample preparation: As received. 


\section{References}

1. Hoz, S.; Livenh, M.; Cohen, D. J. Am. Chem. Soc. 1987, 109 (17), 5149-5156.

2. Busacca, C. A.; Farber, E.; DeYoung, J.; Campbell, S.; Gonnella, N. C.; Grinberg, N.; Haddad, N.; Lee, H.; Ma, S.; Reeves, D.; Shen, S.; Senanayake, C. H. Org. Lett. 2009, 11, 5594-5597.

3. Busacca, C. A.; Grossbach, D.; So, R. C..; O’Brien, E. M.; Spinelli, E. M. Org. Lett. 2003, 5 (4), 595-598.

4. Busacca, C. A.; Milligan, J. A.; Rattanangkool, E.; Ramavarapu, C.; Chen, A.; Saha, A.; Li, Z.; Lee, H.; Geib, S. J.; Wang, G.; Senanayake, C. H. J. Org. Chem. 2014, 79, 9878-9887.

5. Hall, H. K.; Blanchard, E. P.; Cherkofsky, S. C.; Sieja, J. B.; Sheppard, W. A. J. Am. Chem. Soc. 1971, 93 (1), 110-120. 


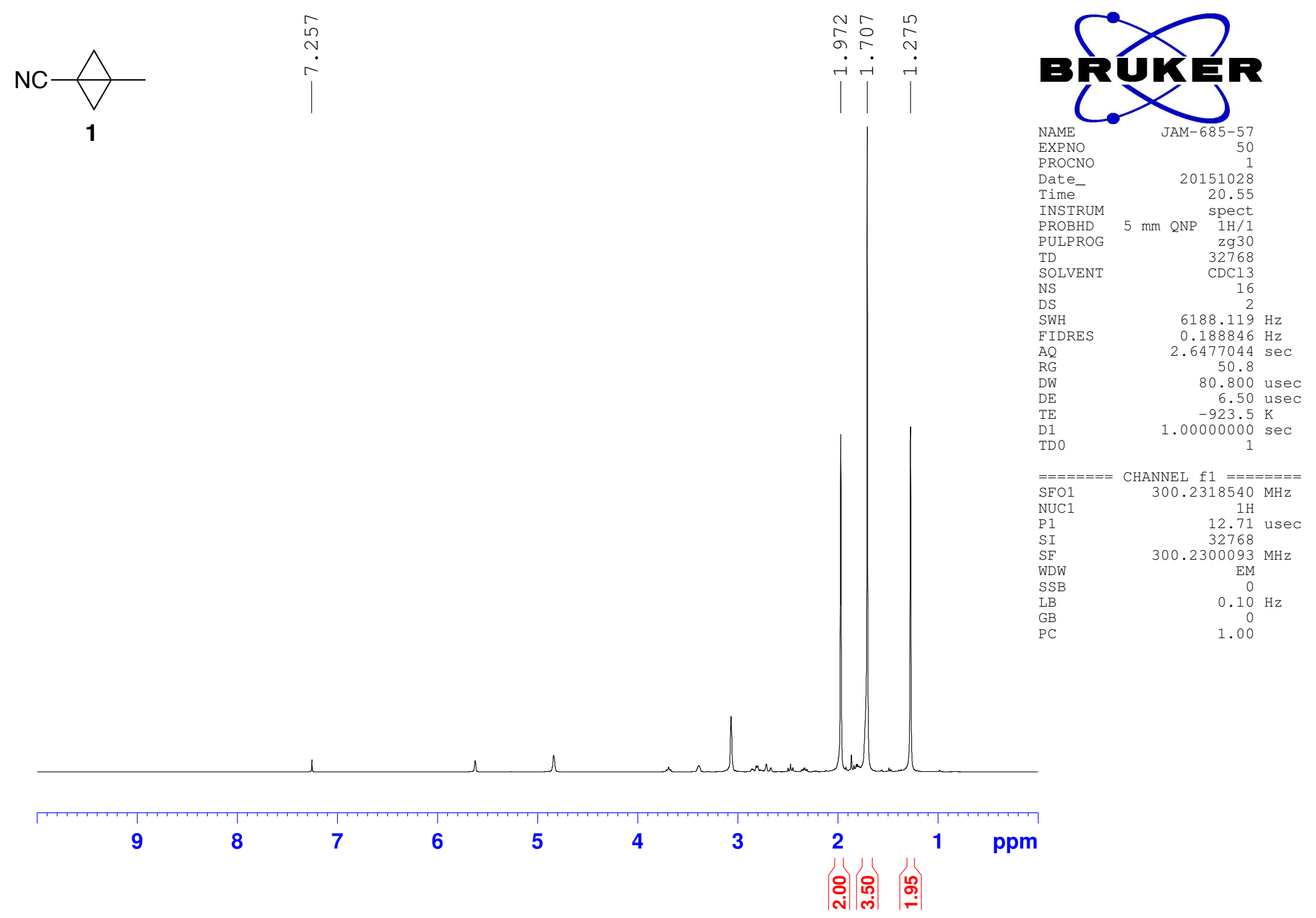




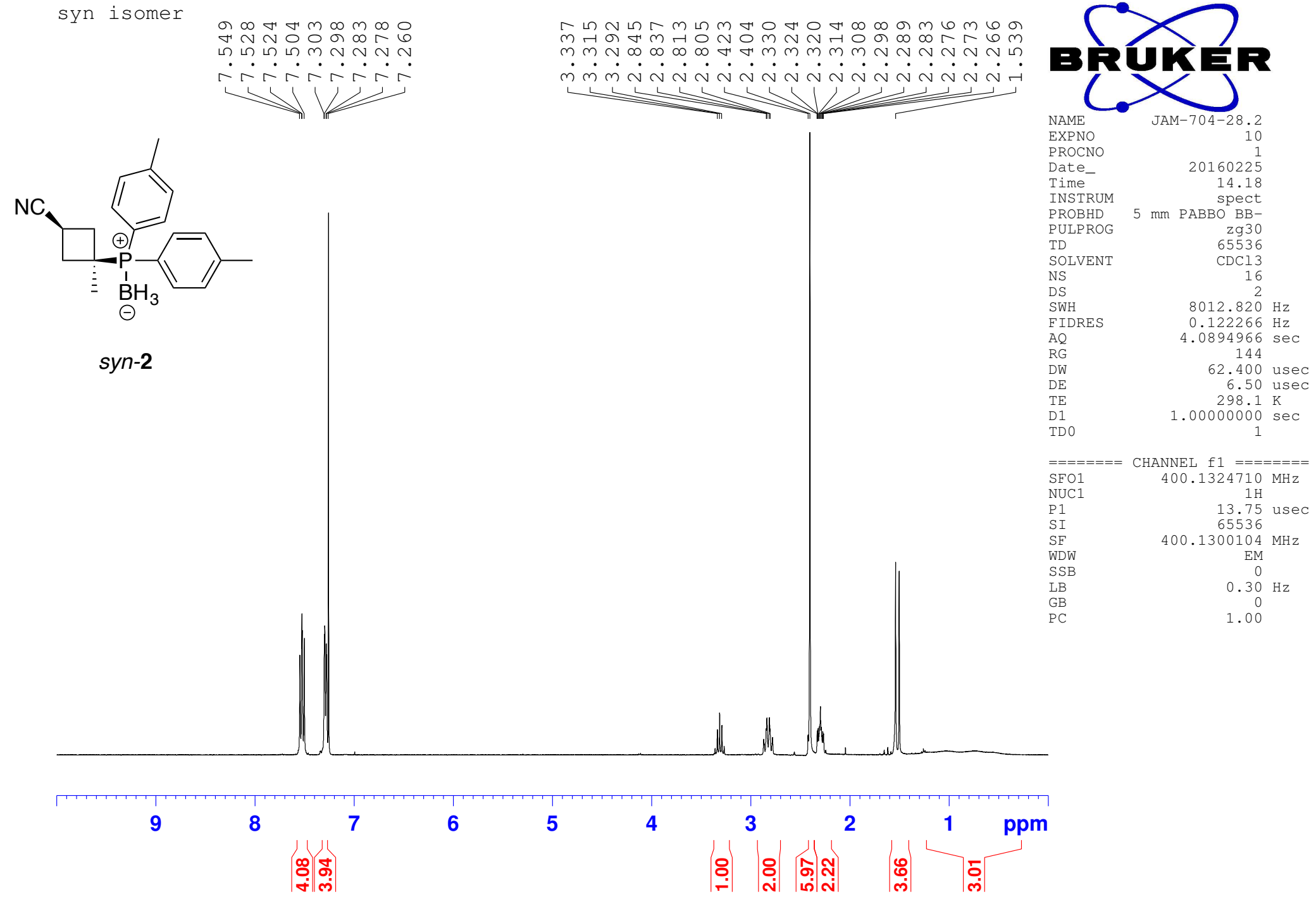




\section{cis isomer recrystallized BRyer}

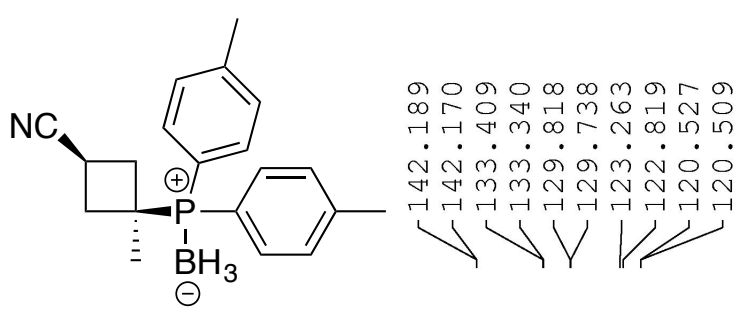

syn-2

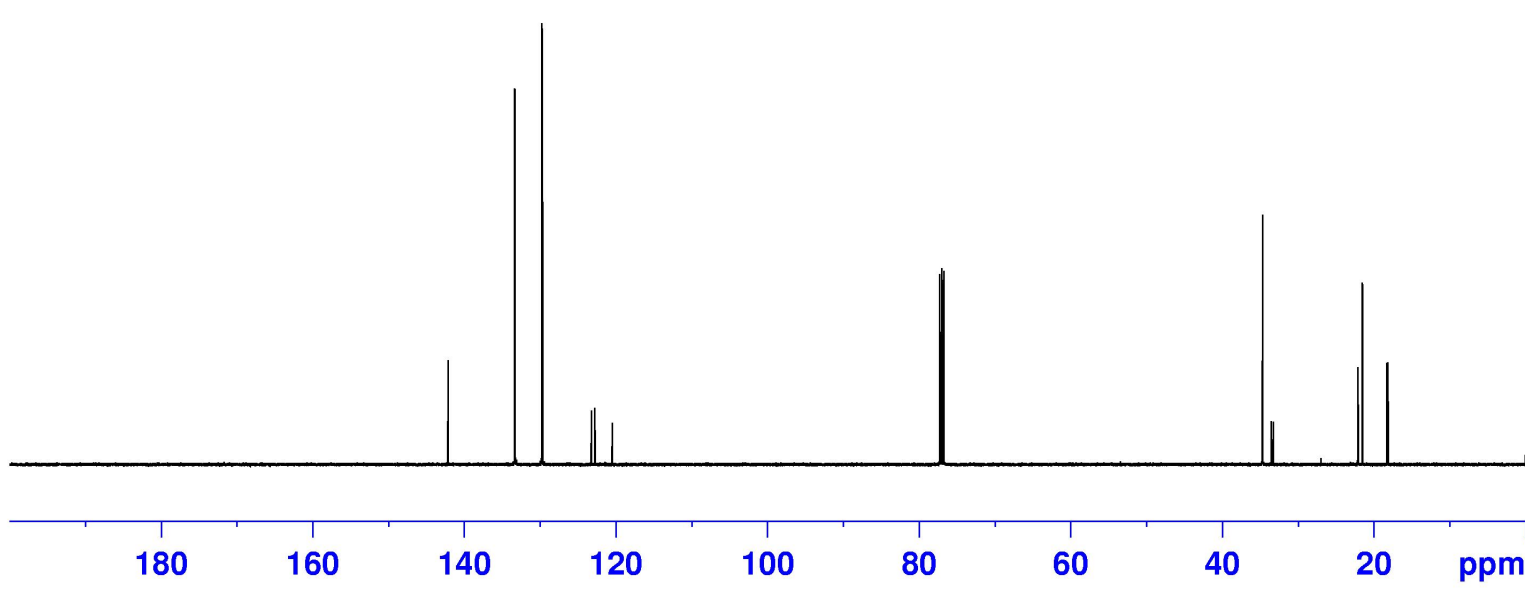

Current Data Parameters EXPNO

mみเn

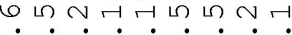

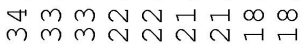

F2 - Acquisition Parameters

$\begin{array}{lr}\text { Date- } & 20150820 \\ \text { Time } & 19.41\end{array}$

INSTRUM spect

PROBHD

TD

SOLVENT
NS

$\begin{array}{lr}\text { DS } & 102 \mathrm{H} \\ \text { SWH } & 31250.000 \mathrm{~Hz} \\ \text { FIDRES } & 0.119209 \mathrm{~Hz}\end{array}$

FIDRES $4.1943040 \mathrm{sec}$

AQ
RG
DW

16.000 use

6.50 usec

$1.00000000 \mathrm{sec}$

$0.03000000 \mathrm{sec}$

$0.89999998 \mathrm{sec}$

125.7698617 MHz 9.75 use

1.00000000 usec $500.1325007 \mathrm{MHz}$

$$
\text { waltz16 }
$$

$\begin{array}{lr}\text { CPDPRG [2 } & \text { waltz16 } \\ \text { PCPD2 } & 80.00 \text { usec }\end{array}$

-1.00000000 us

PLW12 $-1.00000000 \mathrm{~W}$

F2 - Processing parameters

SI $\quad 125.7577899 \mathrm{MHz}$

WDW

$\begin{array}{lll}\text { LB } & 0 & 0.30 \mathrm{~Hz}\end{array}$ 


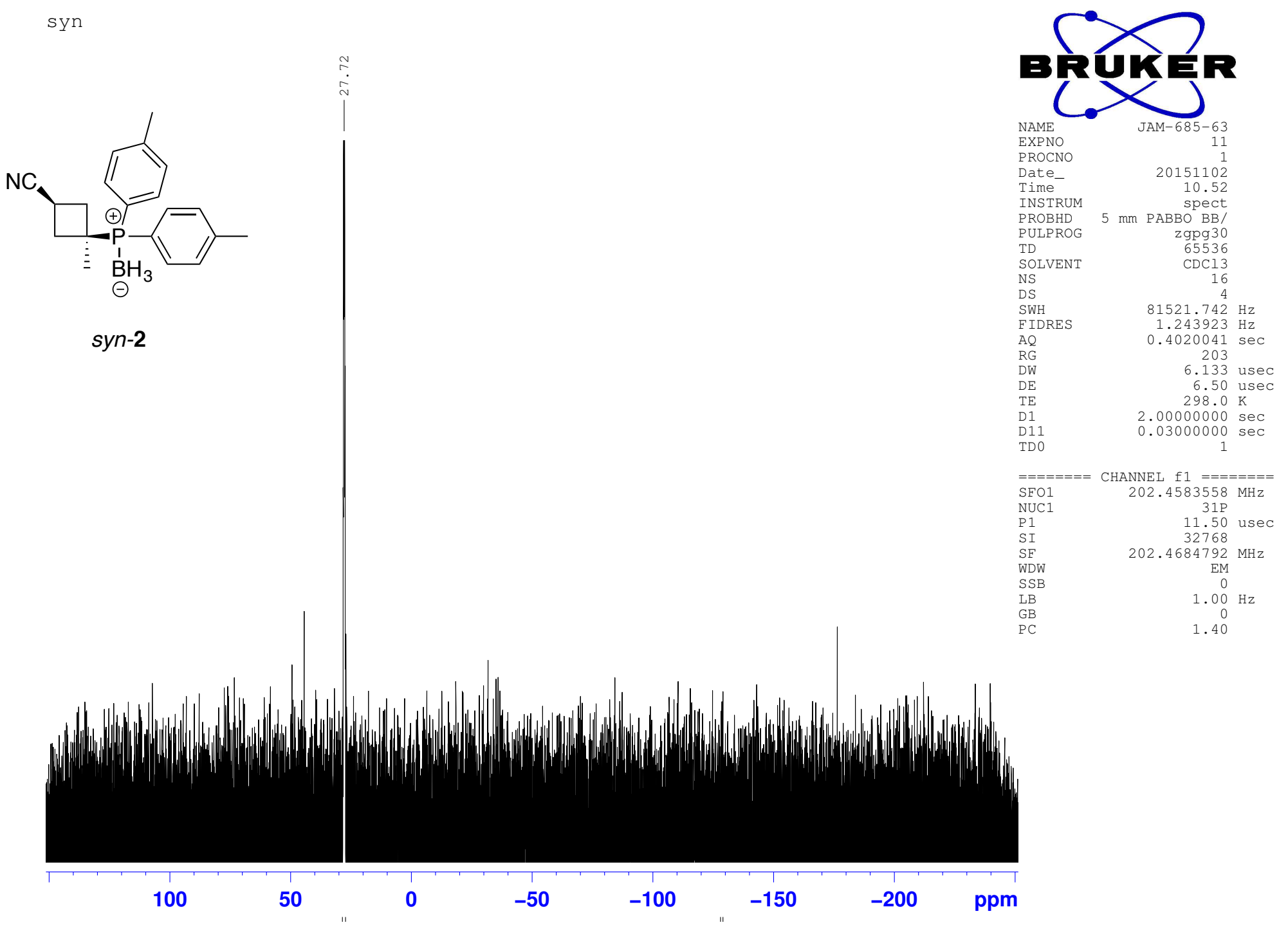




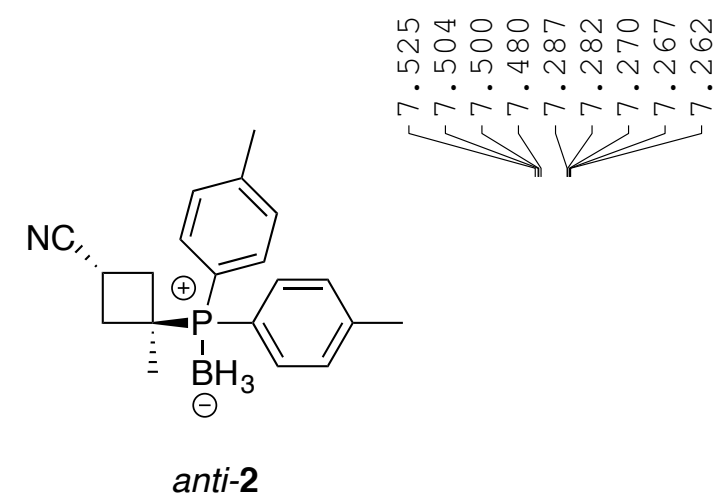

๙ न $\dot{m} \dot{m} \dot{m} \dot{m} \dot{m} \dot{\sim} \dot{\sim} \dot{\sim} \dot{\sim} \dot{\sim} \dot{\sim} \dot{\sim} \dot{r}$
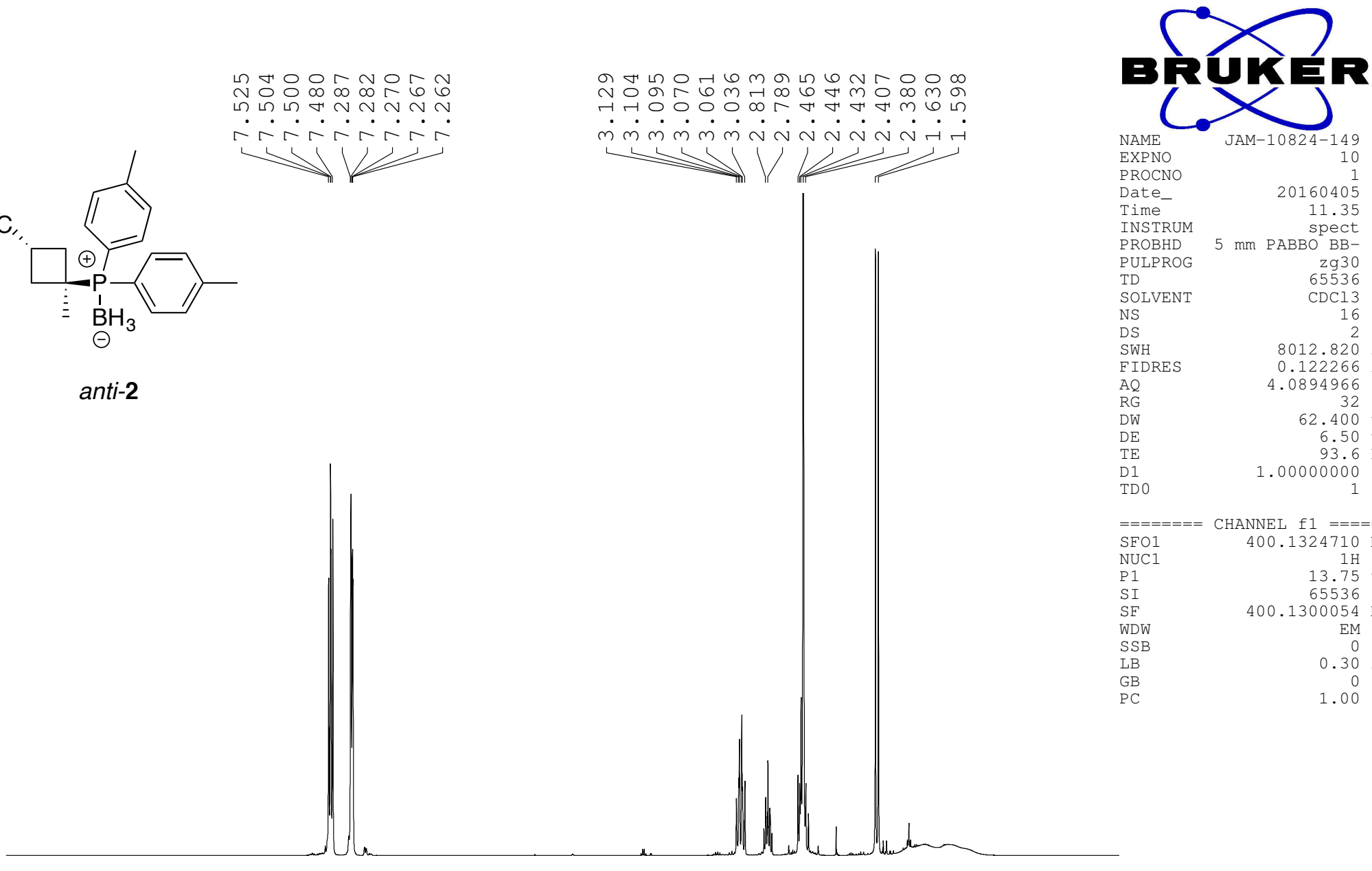

NAME

EXPNO

PROCNO

Date-

INSTRUM

PROBHD

10
10

PULPROG

20160405

zg30
65536

SOLVENT

DS

SWH

FIDRE

$\mathrm{AQ}$
$\mathrm{RG}$

RG

DW

TE
D1
TD 0

1

$=======$ CHANNEL $\mathrm{f} 1 \quad========$
SFO1
NUC1

$\begin{array}{ll}\mathrm{P} 1 & 1 \mathrm{H} \\ \mathrm{SI} & 13.75 \mathrm{use}\end{array}$

13.75 use
65536

$400.1300054 \mathrm{MHz}$

SE
WDW
SSB

$\mathrm{LB}$
$\mathrm{GB}$

EM
0
$0.30 \mathrm{~Hz}$
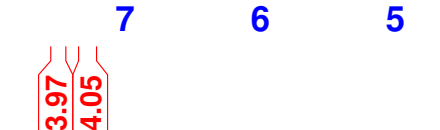

43

농

2

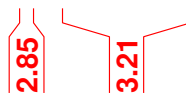

0 ppm

1.00 
$\mathrm{Me}$ BCB nitrile tol HPN minor isomer

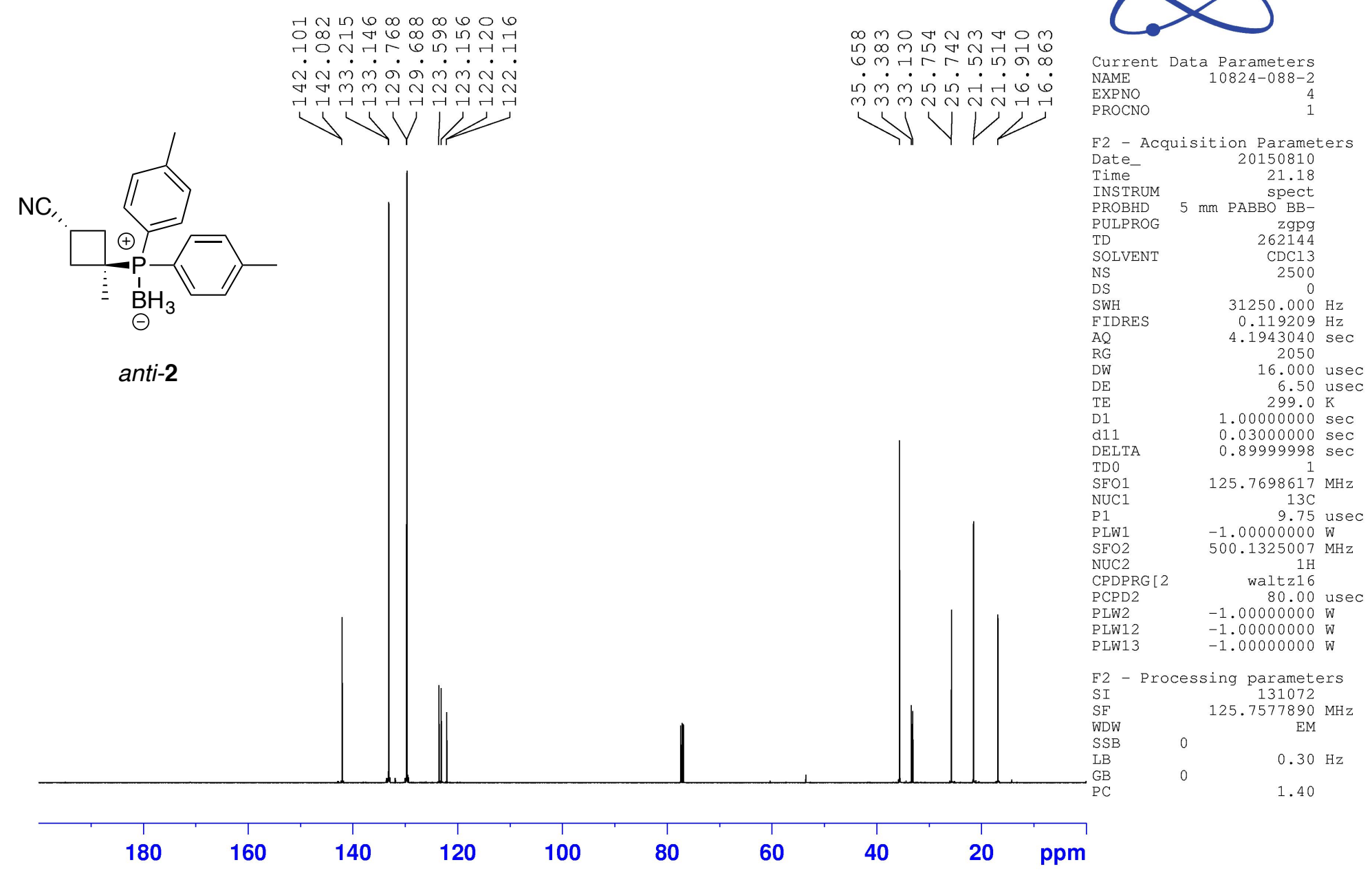


anti isomer

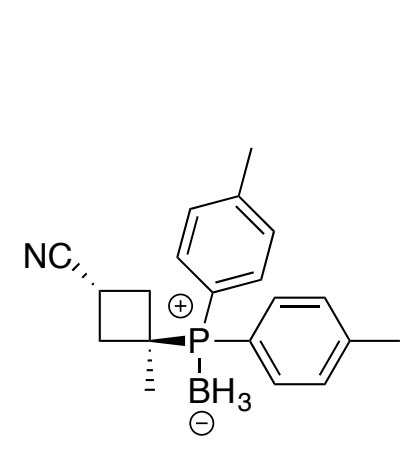

anti-2
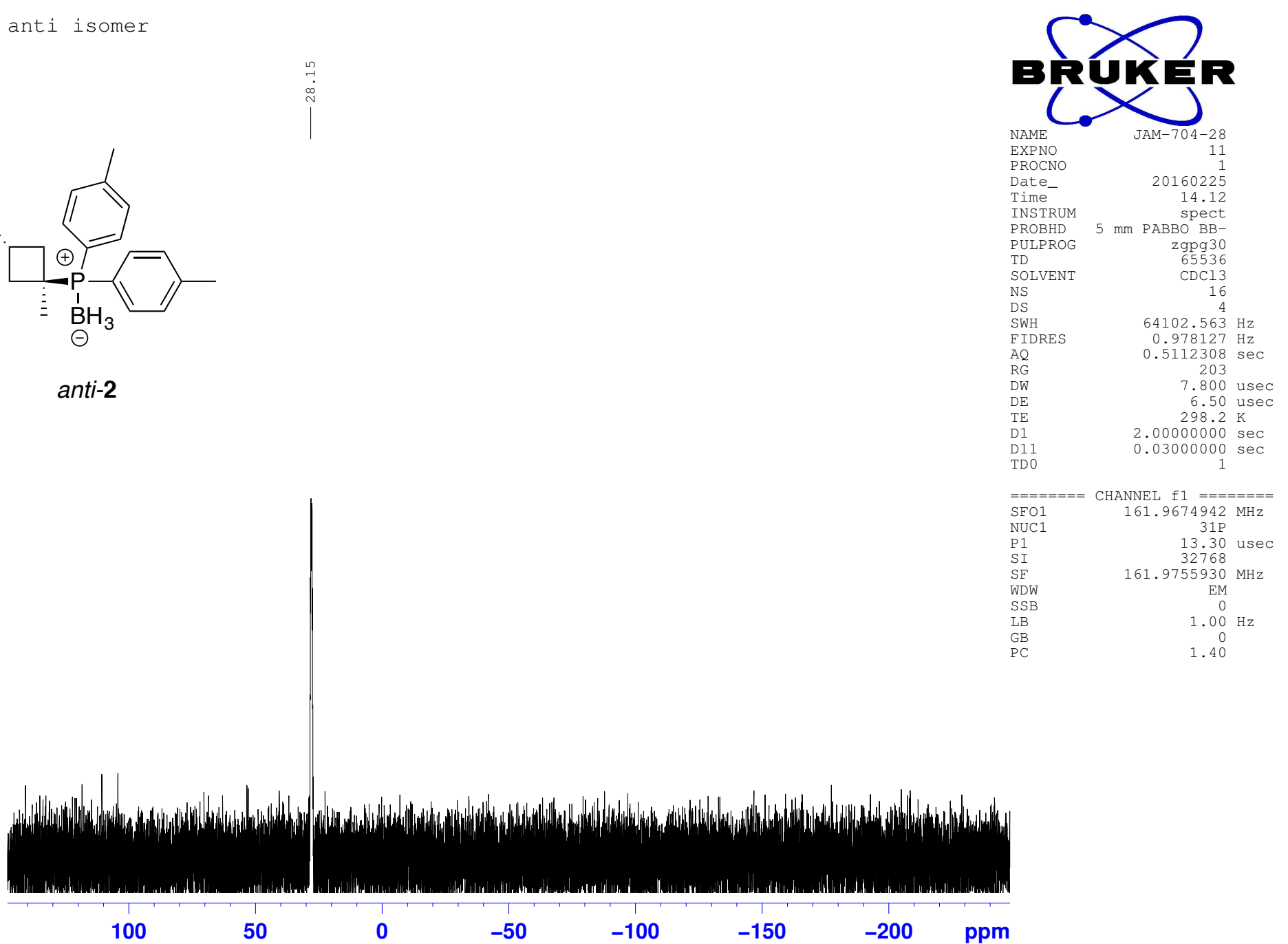

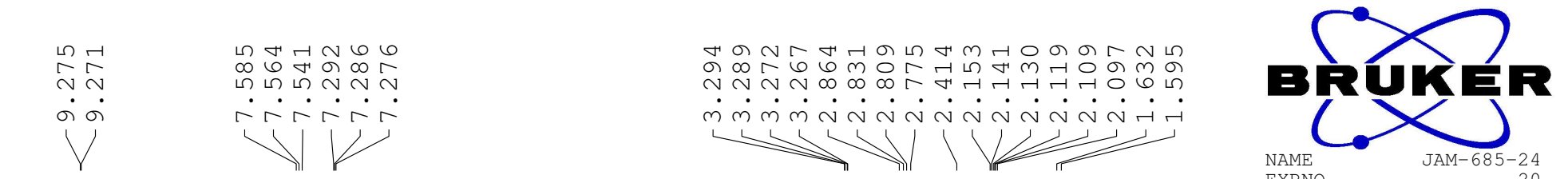

EXPNO

Date

INSTRUM

PROBHD

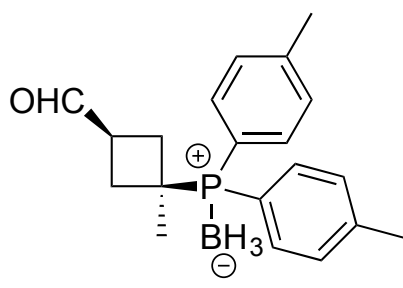

PULPROG

SOLVENT

NS
DS

$\begin{array}{r}16 \\ 2 \\ \hline\end{array}$

$\begin{array}{ll}\text { SWH } & 8012.820 \mathrm{~Hz}\end{array}$

AQ $\quad 4.0894966 \mathrm{sec}$

62.400 use

6.50 usec

TE $\quad 96.7 \mathrm{~K}$

3

D1 0
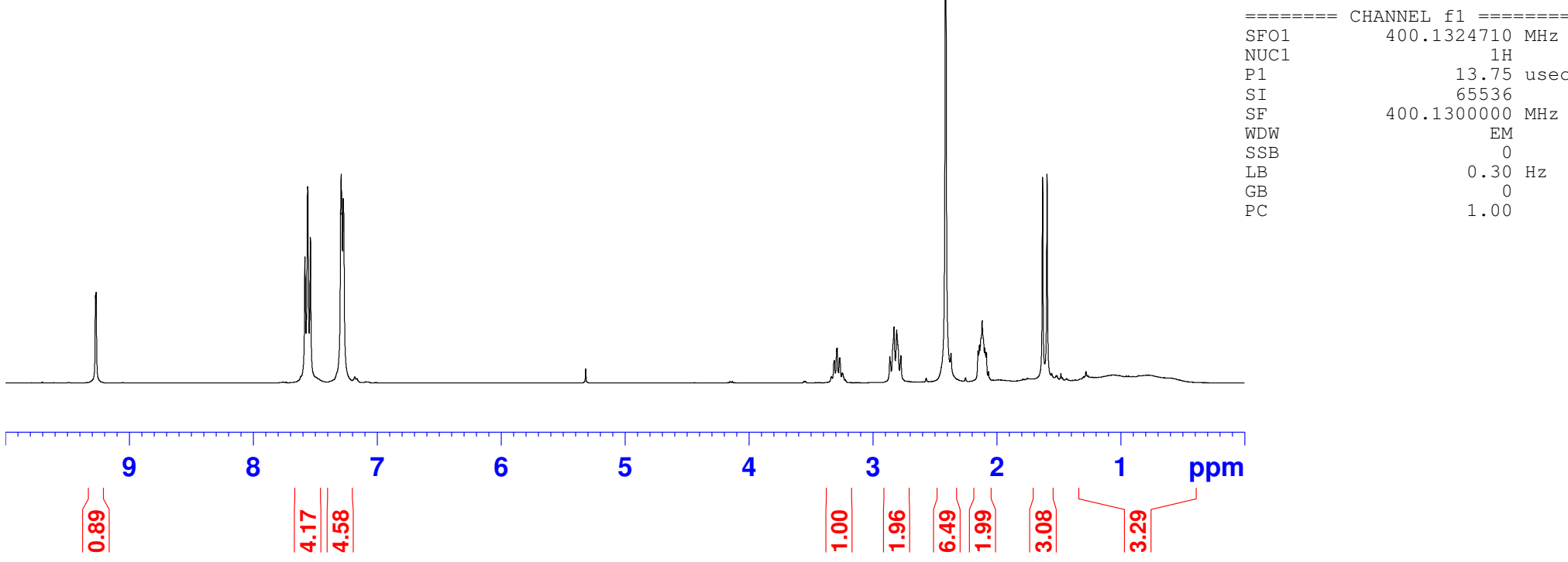


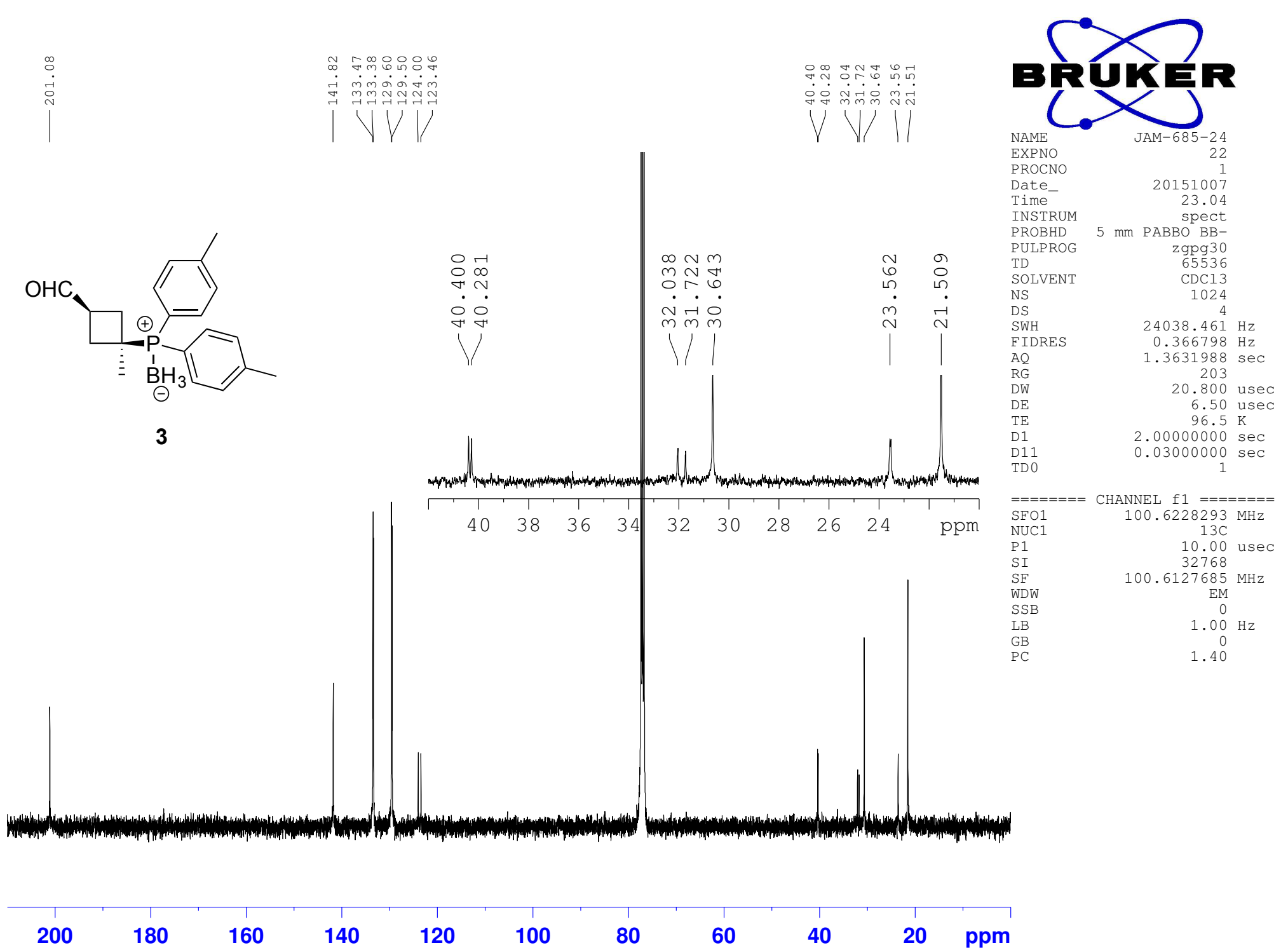



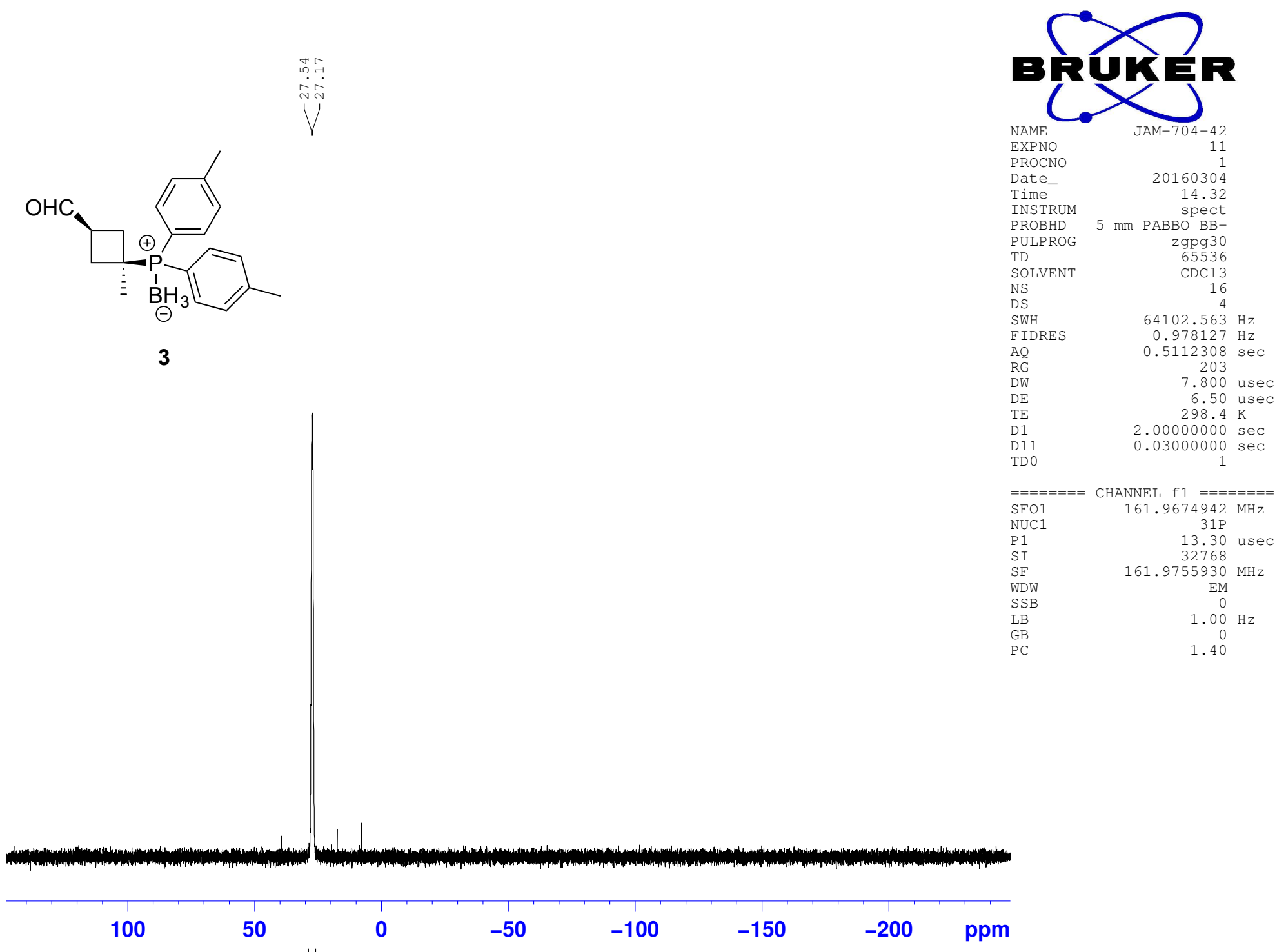


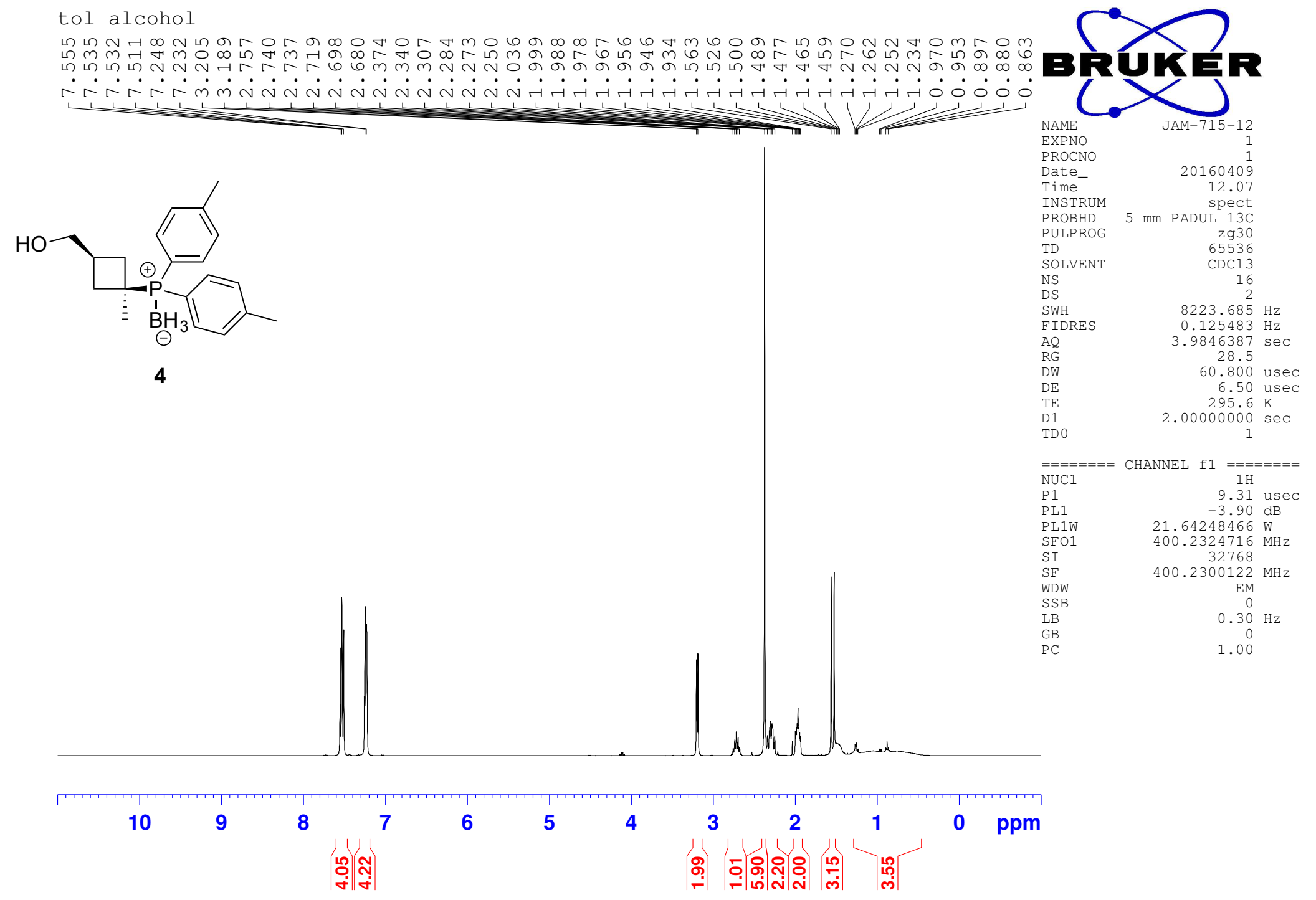


tol alcohol

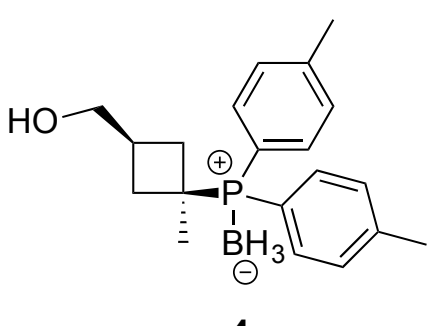

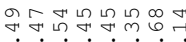

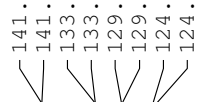

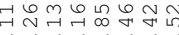

मूलिएल

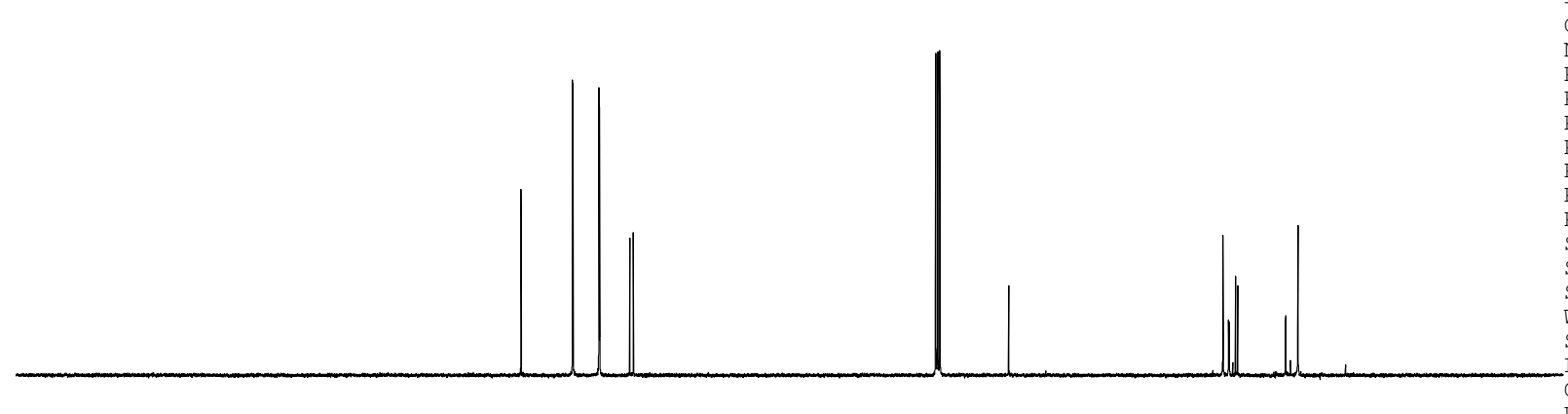

(2)

EXPNO

Date

INSTRUM

$5 \mathrm{~mm}$ PADUL $13 \mathrm{C}$

PULPROG

SOLVEN

NS

$\begin{array}{lr}\text { DS } & 4 \\ \text { SWH } & 24038.461 \mathrm{~Hz} \\ \text { FIDRES } & 0.366798 \mathrm{~Hz} \\ \text { AQ } & 1.3631988 \mathrm{~s}\end{array}$

$\begin{array}{lr}\text { AQ } & 1.3631988 \mathrm{sec} \\ \text { RG } & 71.8\end{array}$

DW

DE

D1
D11

20.800 usec

6.50 usec
6.50 usec

$3.000000 .8 \mathrm{~K}$

$0.03000000 \mathrm{sec}$

$=======$ CHANNEL $\mathrm{f}$

$\mathrm{P} 1$

PL1

PL1W

$13 \mathrm{C}$

$9.19395828 \mathrm{~dB}$

$100.6479773 \mathrm{MHz}$

$=======$ CHANNEL f2 $======$

WPDPRG2 waltz16

NUC2

$\mathrm{PCPD} 2$

$\mathrm{PL} 2$
$\mathrm{PL} 12$

L13

(

PL13W

SFO2

SF
WDW

LB

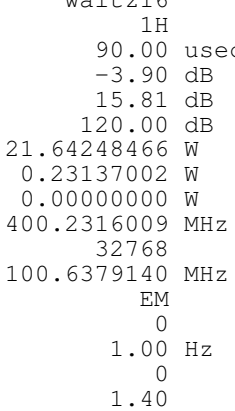




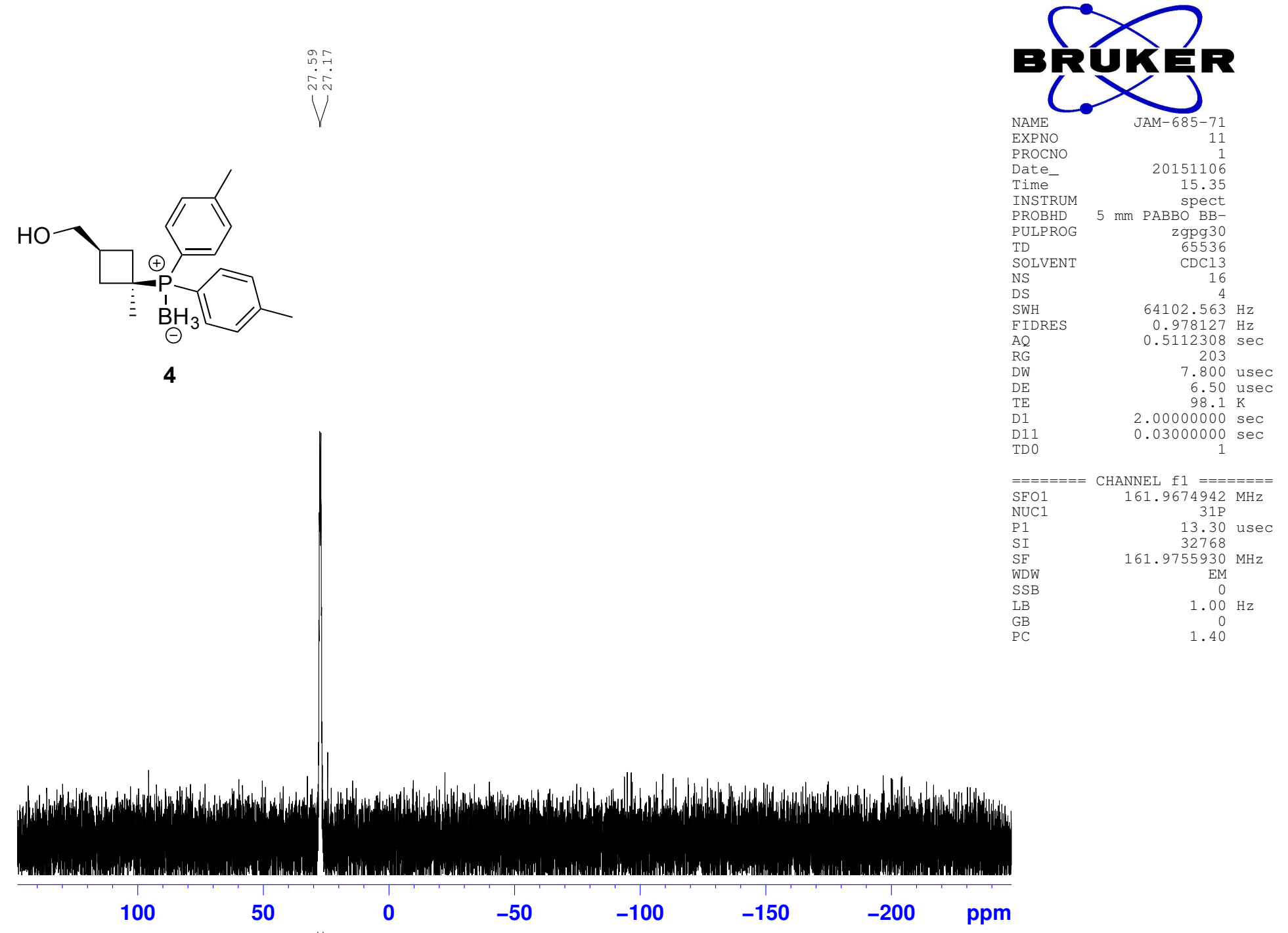




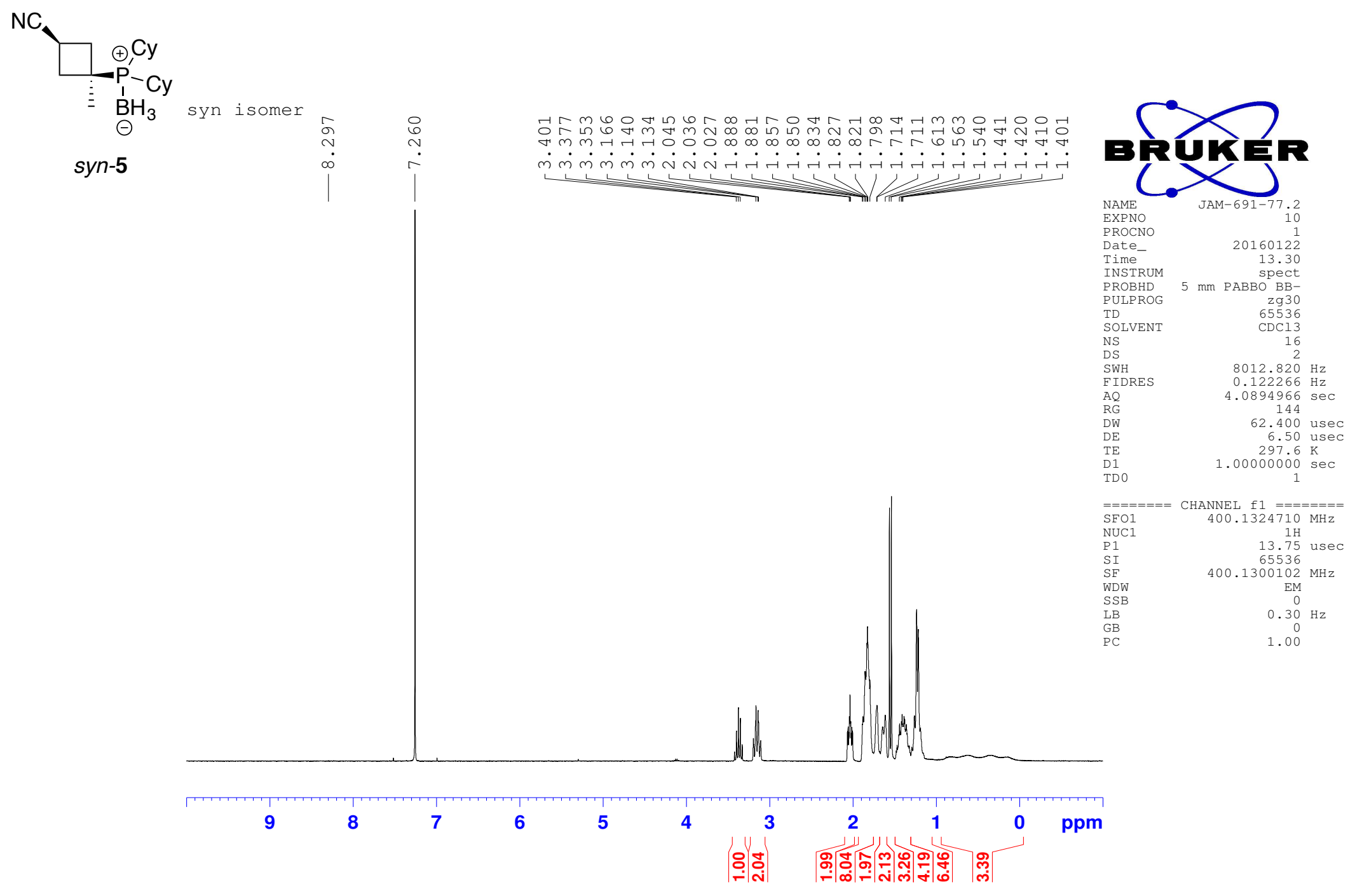


syn isomer

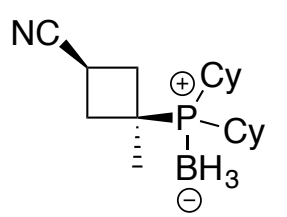

syn-5
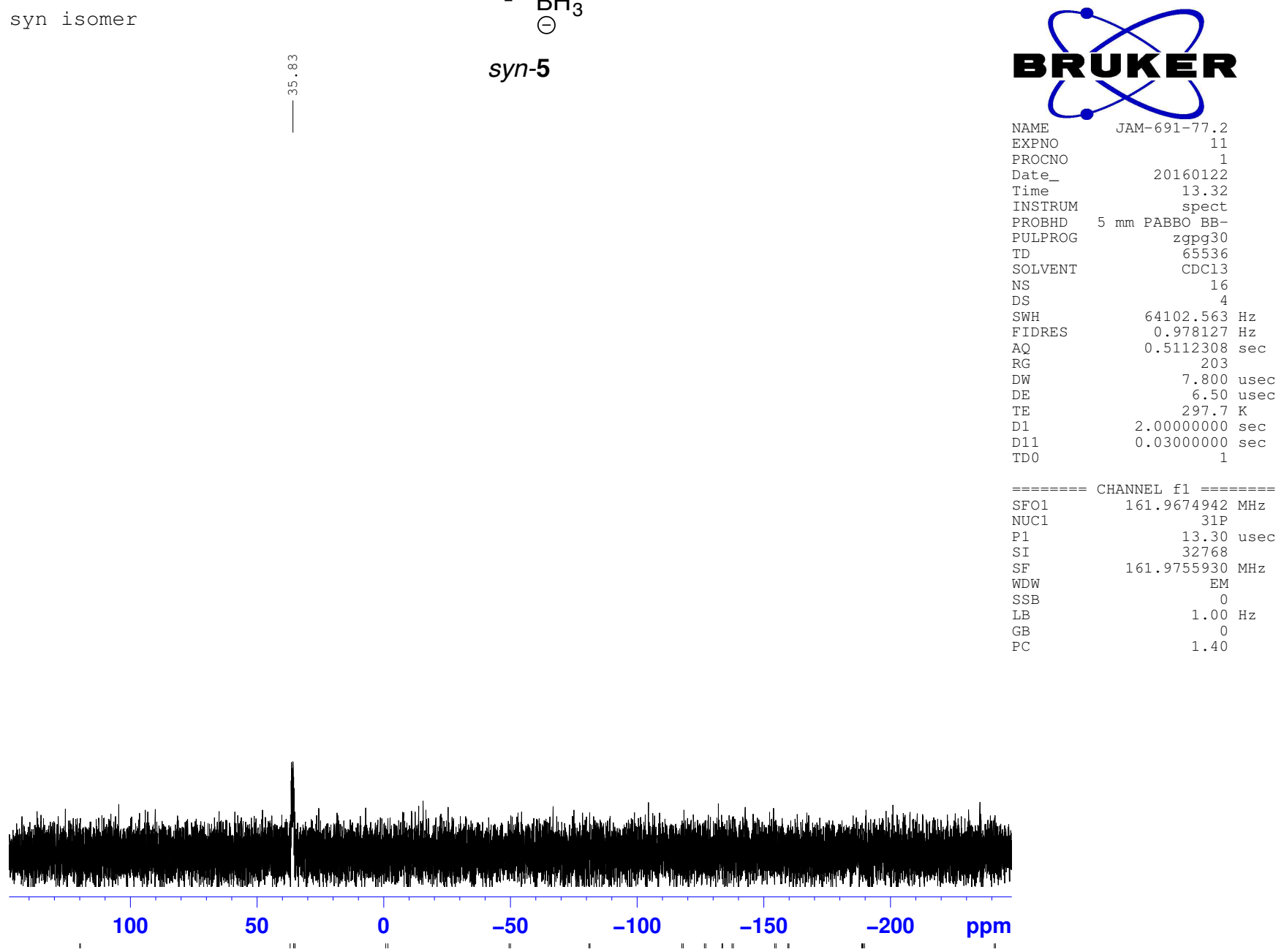


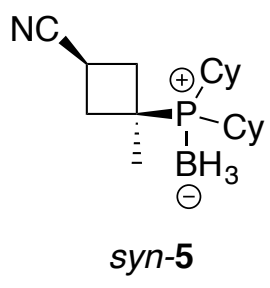

\section{HPN bottom spot CDCl3}

\section{apingen $\longrightarrow$}

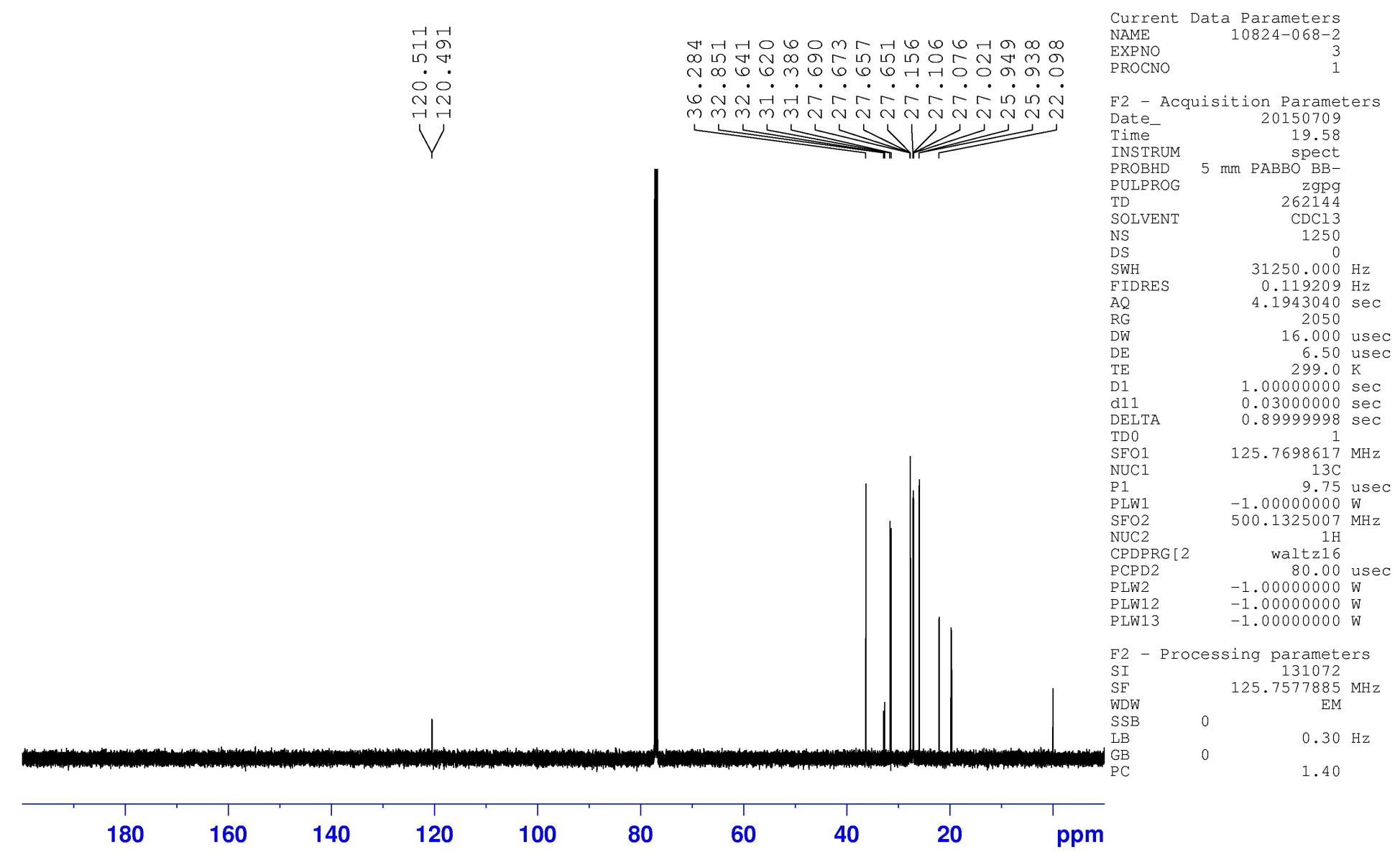



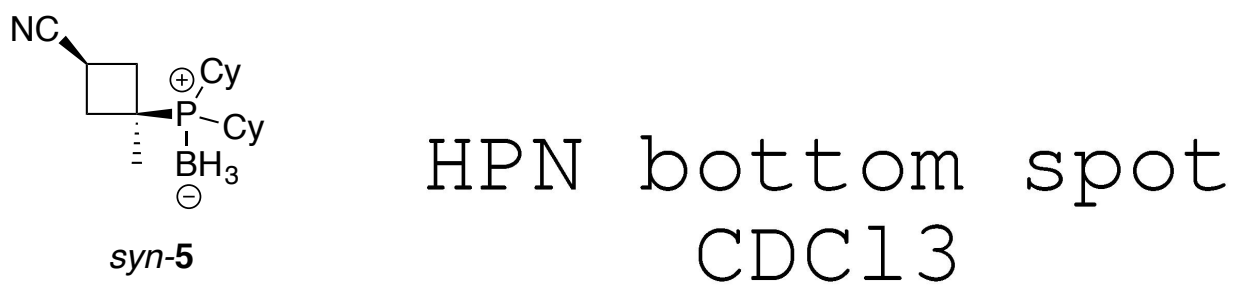

\section{arun}

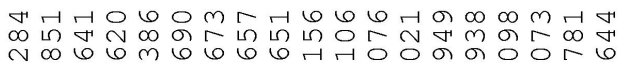

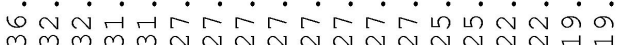

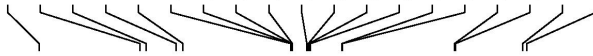

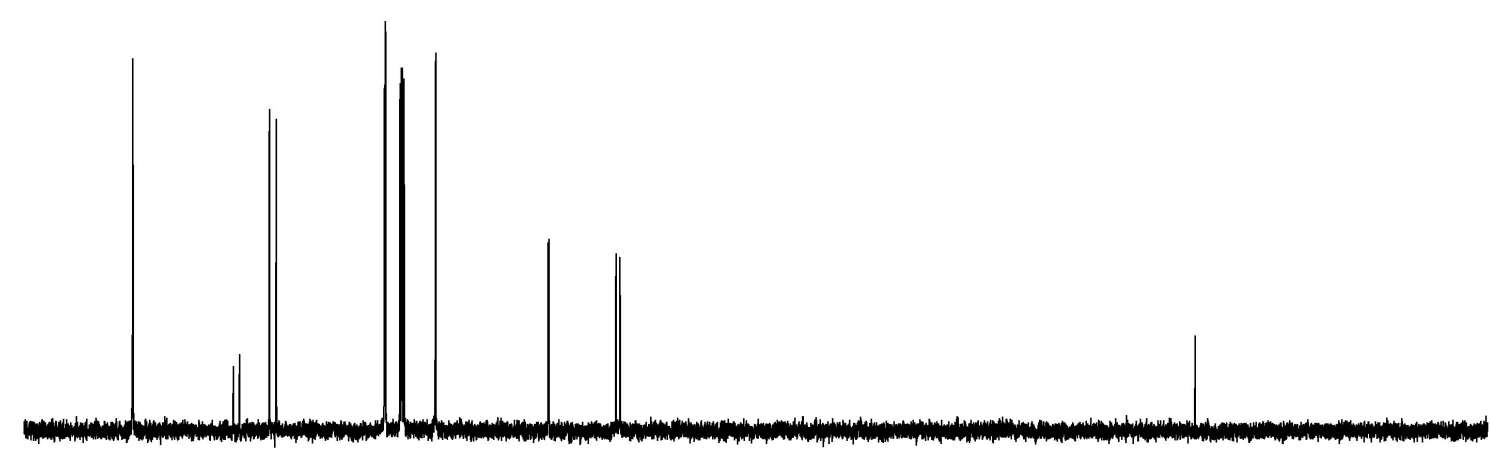

Current Data Parameters
NAME
$10824-068-2$

PROCNO

F2 - Acquisition Parameters

$\begin{array}{lr}\text { Date_- } & 20150709 \\ \text { Time } & 19.58\end{array}$

Spect
INSTRUM
PROBHD $5 \mathrm{~mm}$ PABBO BB-

$\begin{array}{lr}\text { PULPROG } & \text { zgpg } \\ \text { TD } & 262144 \\ \text { SOLVENT } & \text { CDC13 } \\ \text { NS } & 1250\end{array}$

$\begin{array}{ll}\text { SWH } & 31250.000 \mathrm{~Hz}\end{array}$

$\begin{array}{ll}\text { FIDRES } & 0.119209 \mathrm{~Hz} \\ \mathrm{AQ} & 4.1943040 \mathrm{sec}\end{array}$

$\begin{array}{lr}\text { AQ } & 4.1943040 \\ \text { RG } & 2050 \\ \text { DW } & 16.000 \text { usec }\end{array}$

16.000 usec
6.50 usec

$1.00000000 \mathrm{sec}$
$0.03000000 \mathrm{sec}$

$0.89999998 \mathrm{sec}$

$125.7698617 \mathrm{MHz}$

$-1.00000000 \mathrm{~W}$
$500.1325007 \mathrm{MHz}$

500.1325007

Waltz16

PLW12 $-1.00000000 \mathrm{~W}$

$\begin{array}{lc}\text { F2 - Processing parameters } \\ \text { SI } & 131072 \\ \text { SF } & 125.7577885 \\ \text { WDW } & \text { EM }\end{array}$

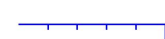

35

30

25

20

15

10

5

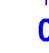

$-5$

ppm 
anti isomer

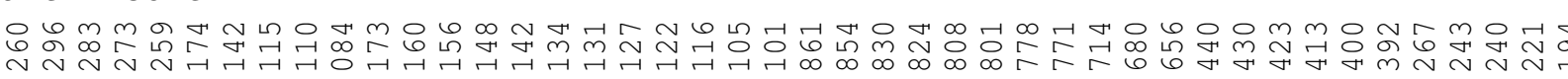

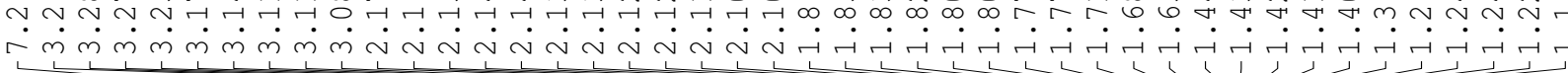

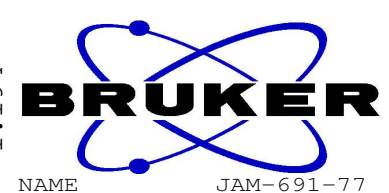

NC

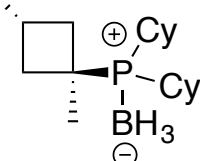

anti-5

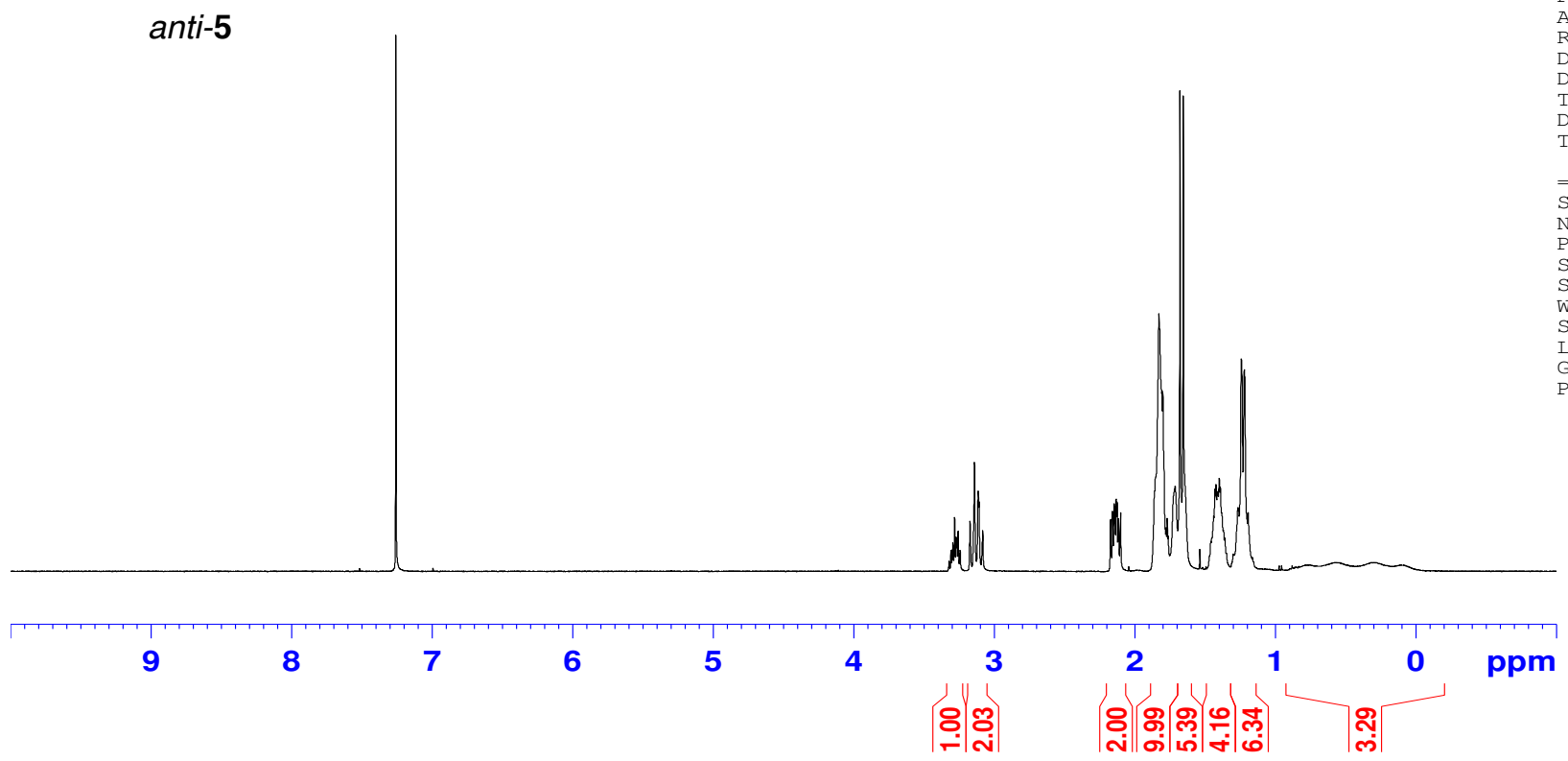


anti isomer
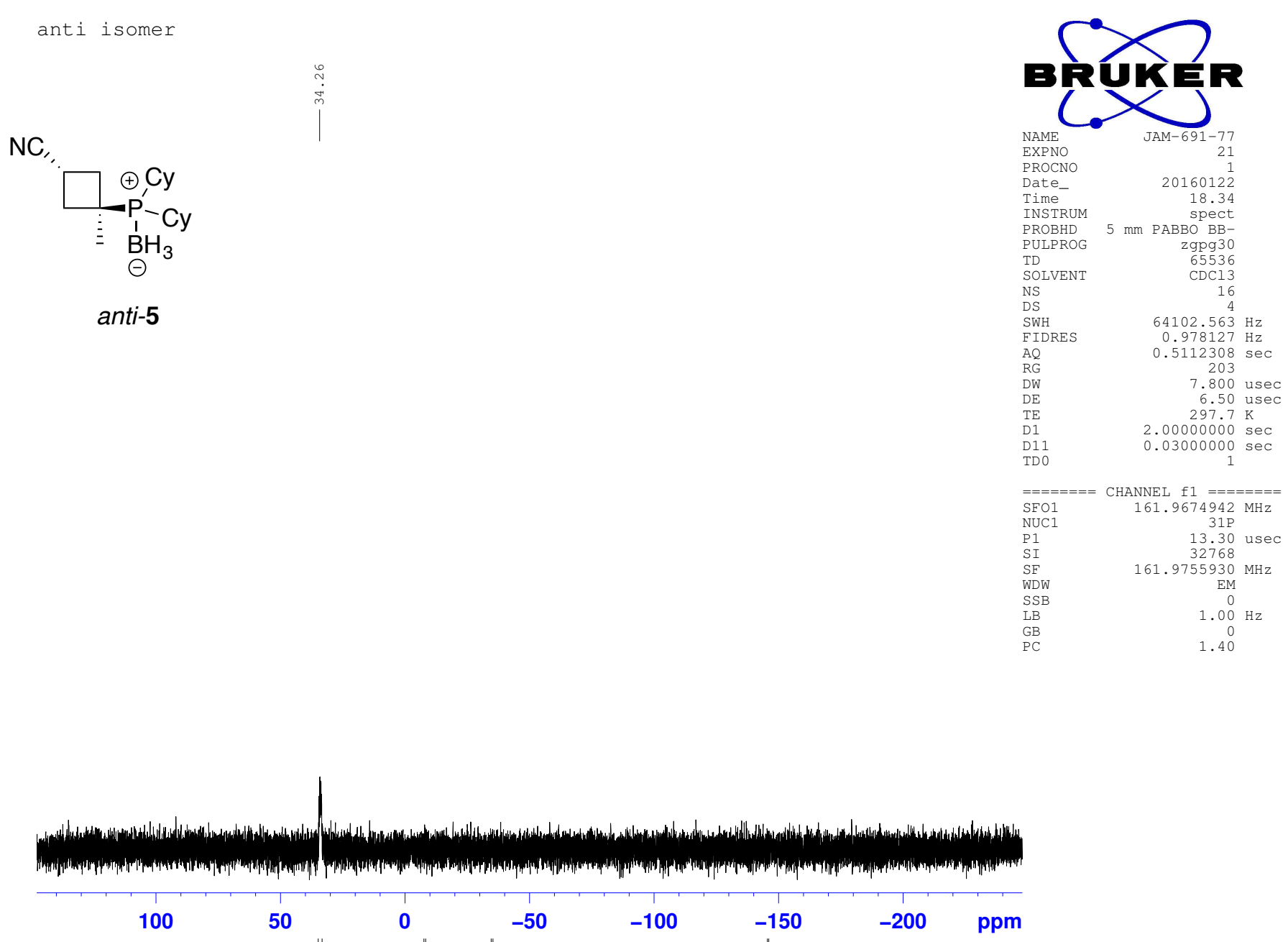
anti isomer

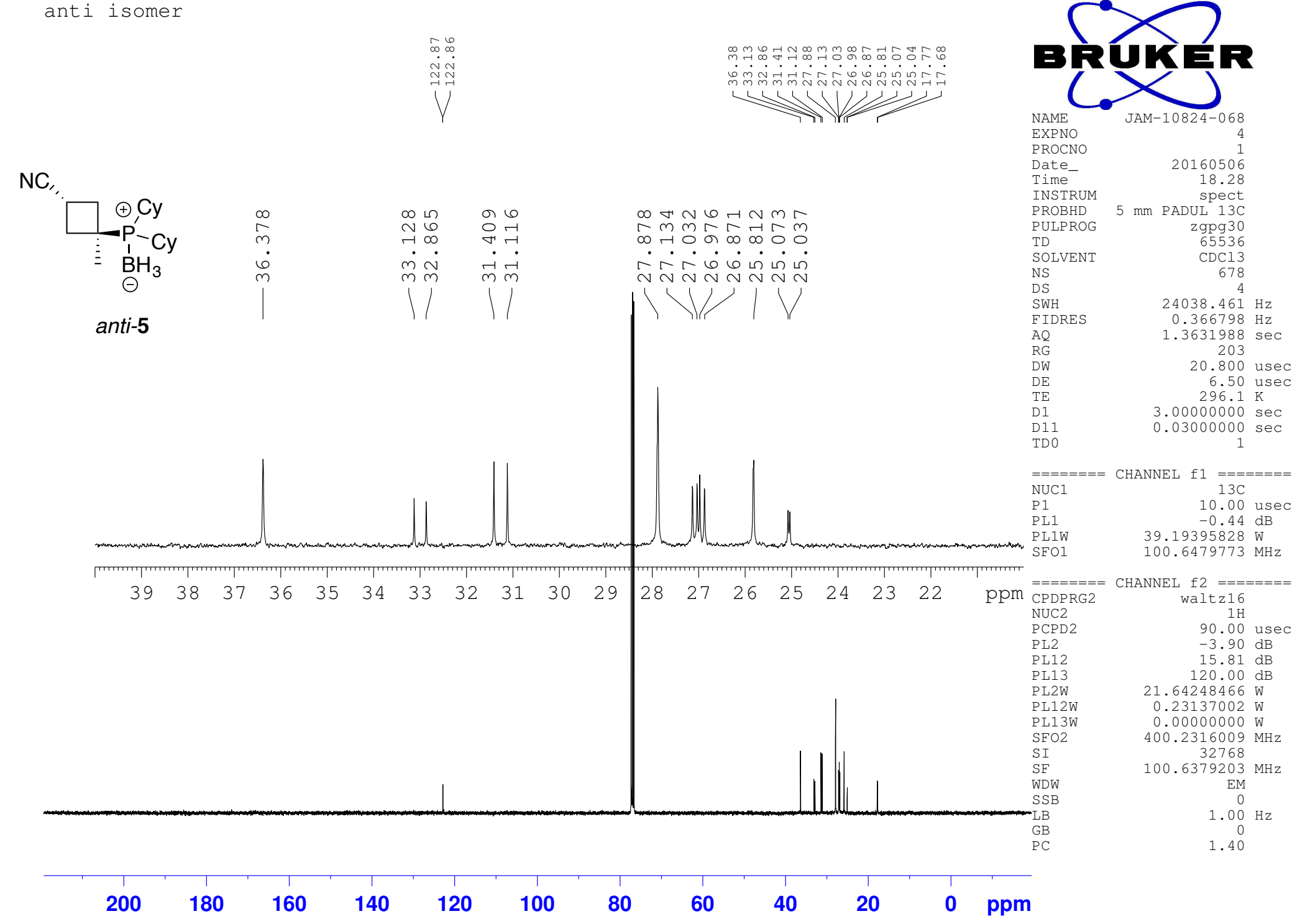




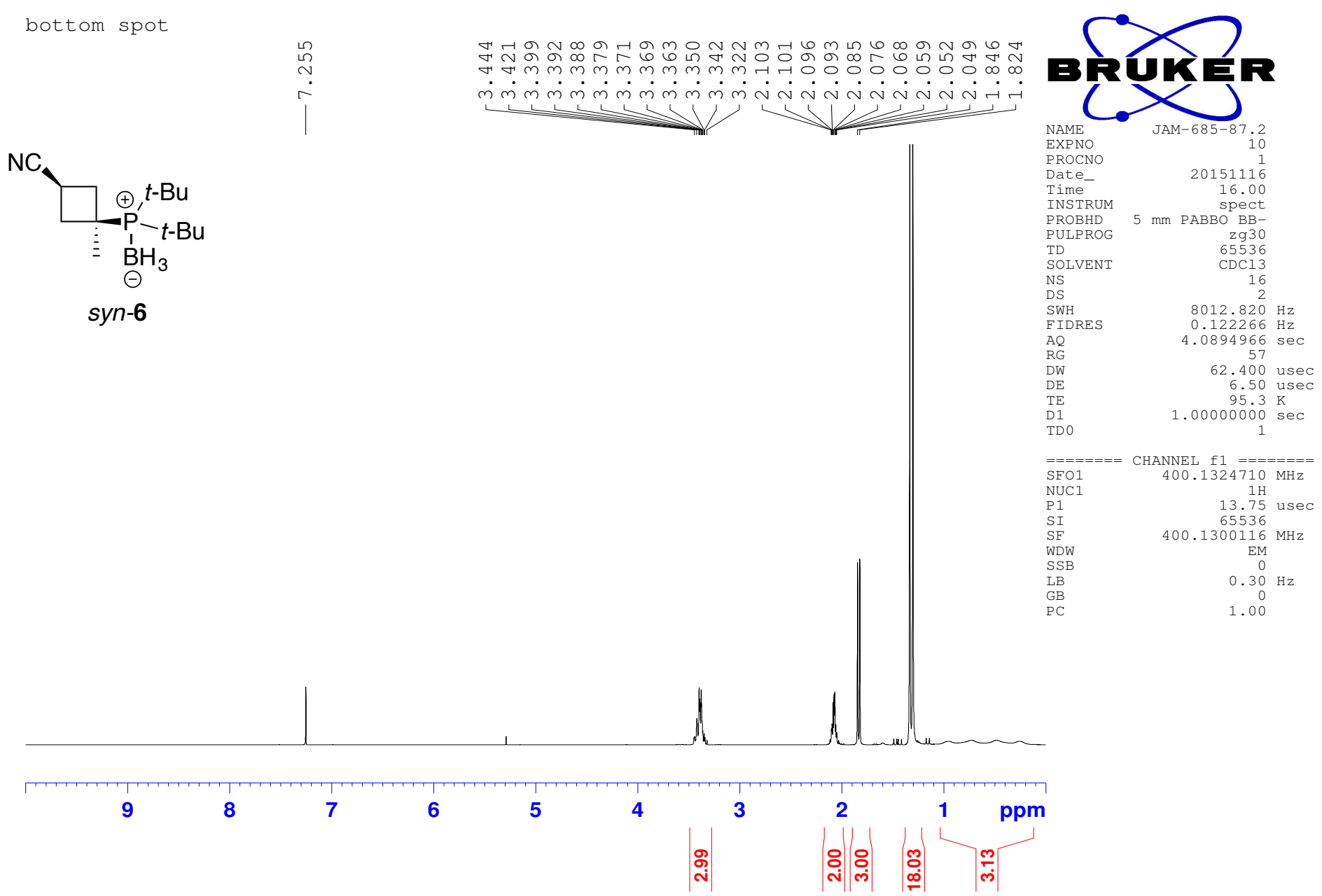


bottom spot
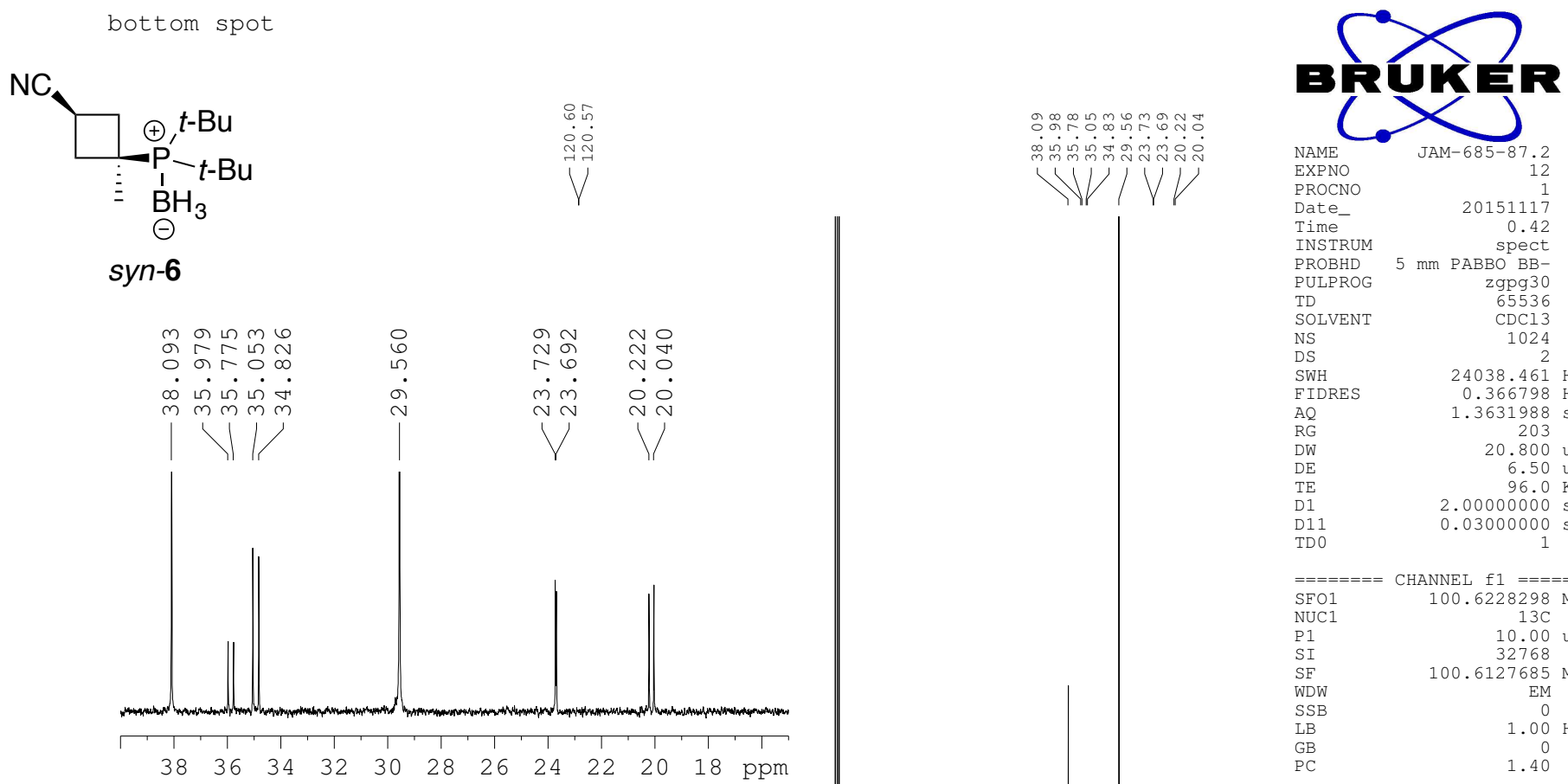

(1)

$\mathrm{BH}_{3}$
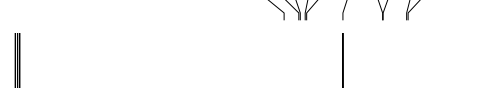

EXPNO

PROCNO
Date

Time
INSTRUM

spect
PROBHD
PULPROG

TD
SOLVENT

DS

SWH

FIDRE
AQ
RG
$D W$

$\mathrm{RG}$
$\mathrm{DW}$
$\mathrm{DE}$

DE
TE
D1
D11
TDO

$1.3631988 \mathrm{sec}$

20.800 usec

6.50 usec
$96.0 \mathrm{~K}$

TD

$2.00000000 \mathrm{sec}$
$0.03000000 \mathrm{sec}$

$\begin{array}{lr}======= & \text { CHANNEL } f 1 \quad======= \\ \text { SFO1 } & 100.6228298 \mathrm{MHz} \\ \text { NUC1 } & 13 \mathrm{MH} \\ \text { P1 } & 10.00 \mathrm{usec} \\ \text { SI } & 32768 \\ \text { SF } & 100.6127685 \mathrm{MHz} \\ \text { WDW } & \text { EM } \\ \text { SSB } & 0 \\ \text { IB } & 1.00 \mathrm{~Hz} \\ \text { GB } & 0 \\ \text { PC } & 1.40\end{array}$

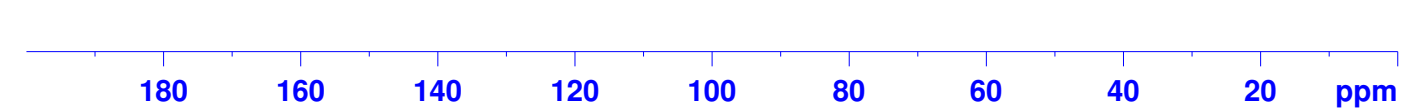


bottom spot
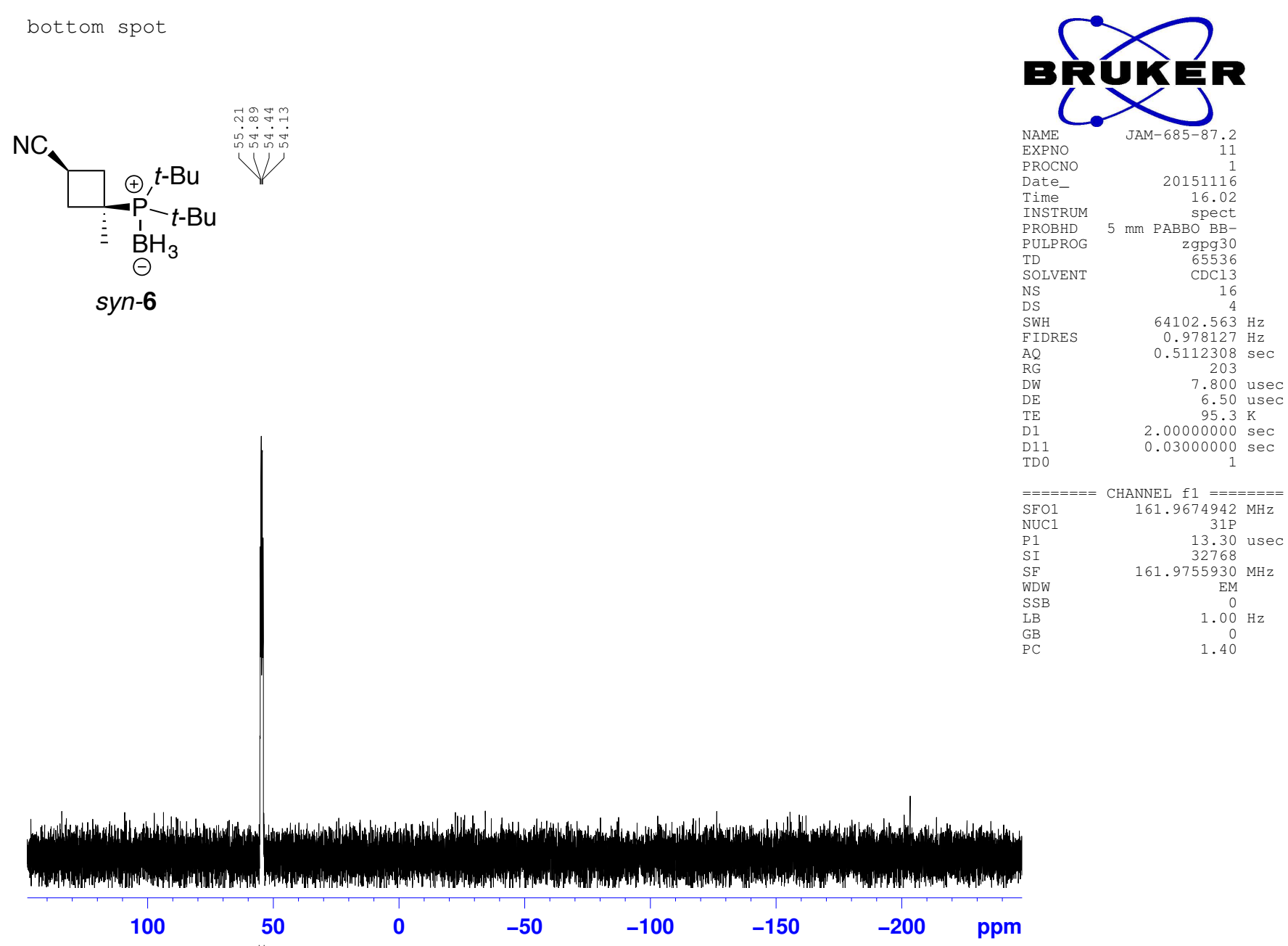
NC,

$$
\begin{aligned}
& \square \stackrel{\oplus}{\oplus}{ }^{t-\mathrm{Bu}} \\
& =\mathrm{BH}_{3} \\
& \text { anti-6 }
\end{aligned}
$$

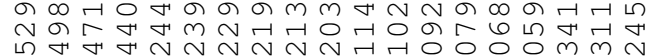

r.

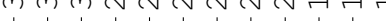
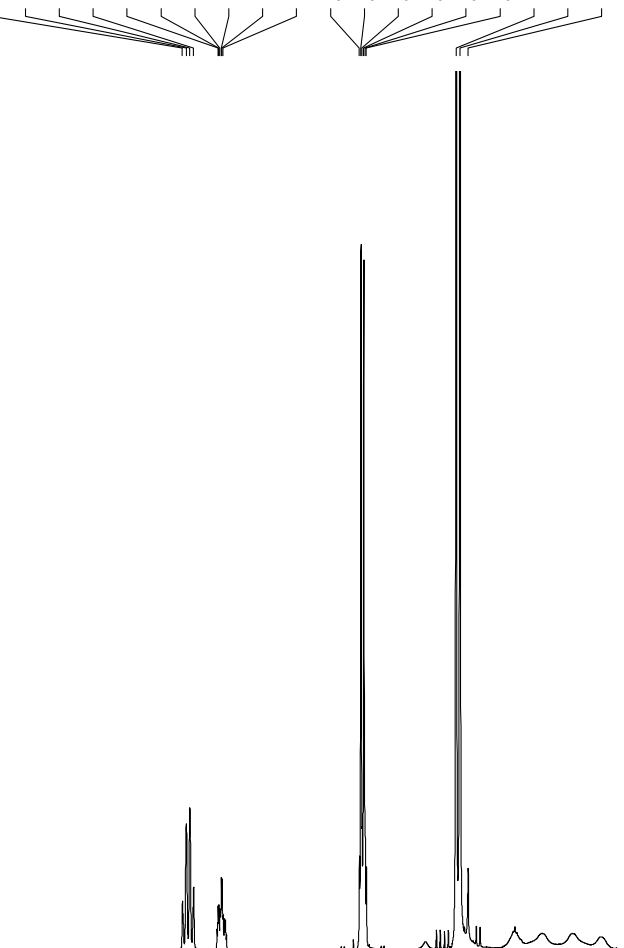

\section{Prukn}
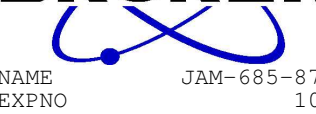

PROC

Date

Time-

$5 \mathrm{~mm}$ PABBO BB-

PULPROG

SOLVENT

NS
DS

FIDRES $\quad 0.122266 \mathrm{~Hz}$

$\mathrm{AQ}$
$\mathrm{RG}$

$\begin{array}{lr}\text { RG } & 80.6 \\ \text { DW } & 62.400 \text { usec } \\ \text { DE } & 6.50 \text { usec }\end{array}$

TE

TDO

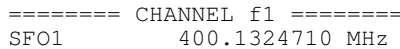

SFO1 $400.1324710 \mathrm{MHZ}$

P1 $1 \mathrm{H}$

$S I$
WDW
SSB

SSB

LB

$400.1300120 \mathrm{MHz}$

EM

0
$0.30 \mathrm{~Hz}$

0.30
0
1.00
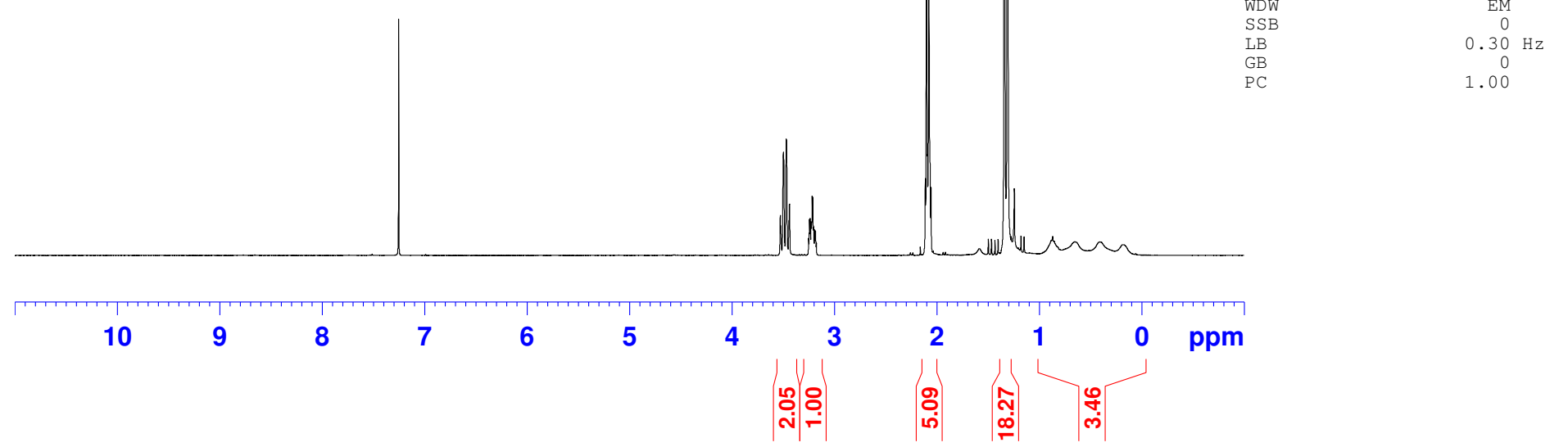
anti

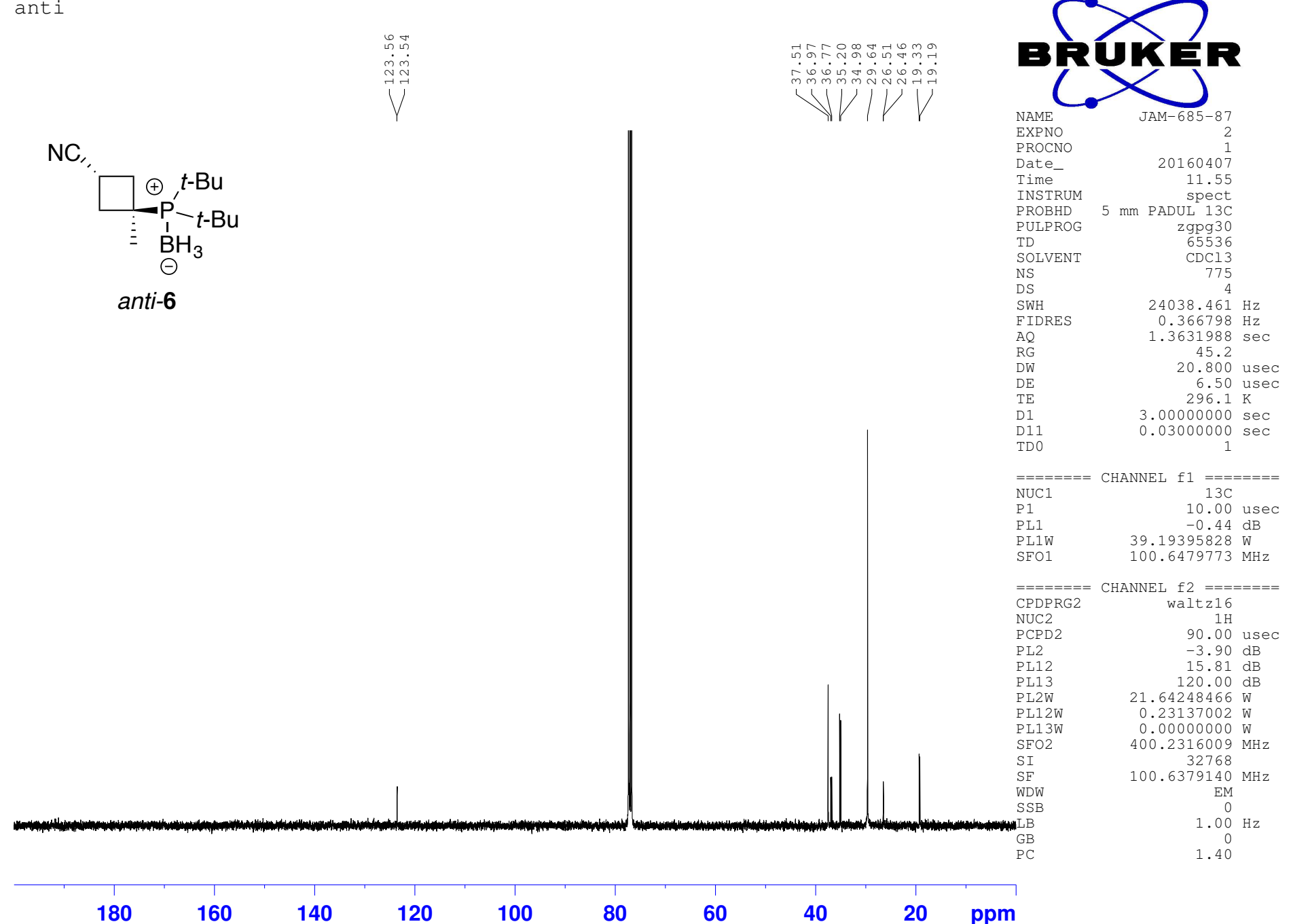


top spot

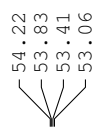

NC

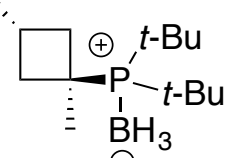

$\Theta$

anti-6
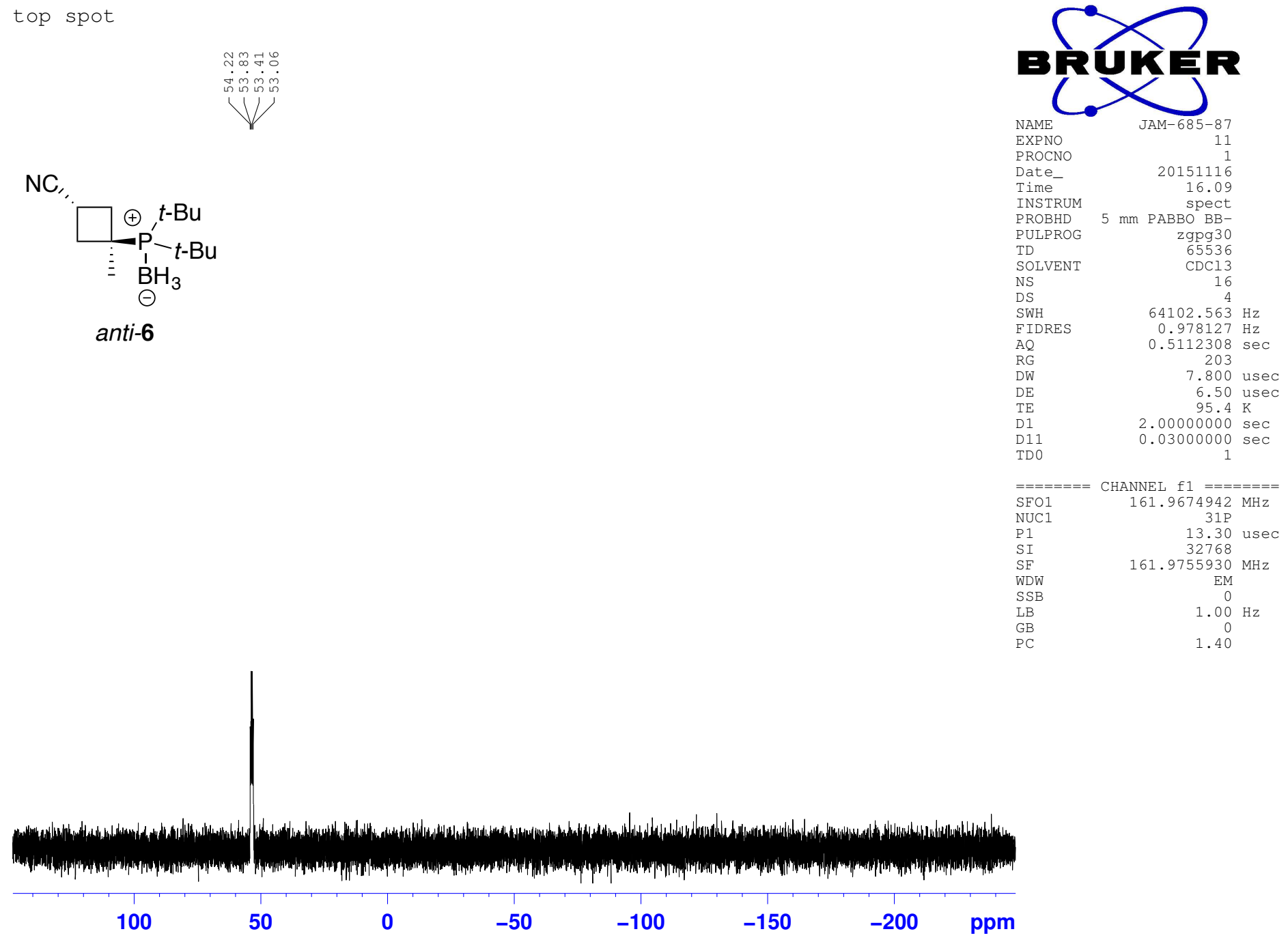
syn isomer
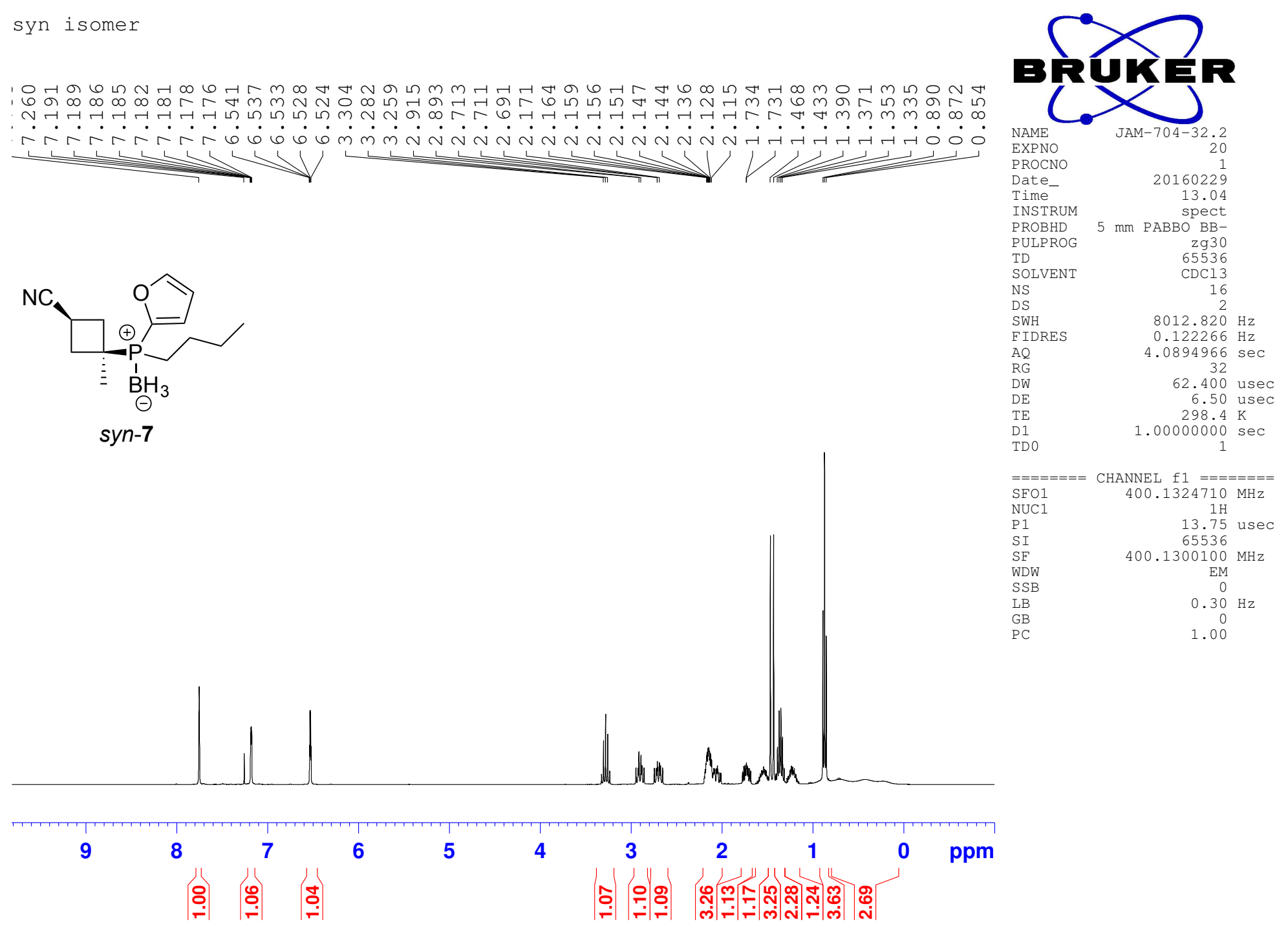

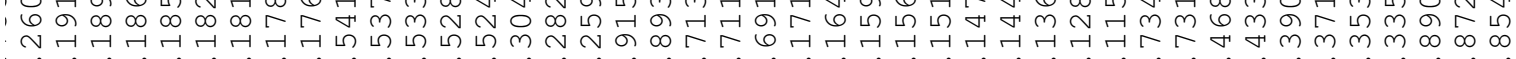
N
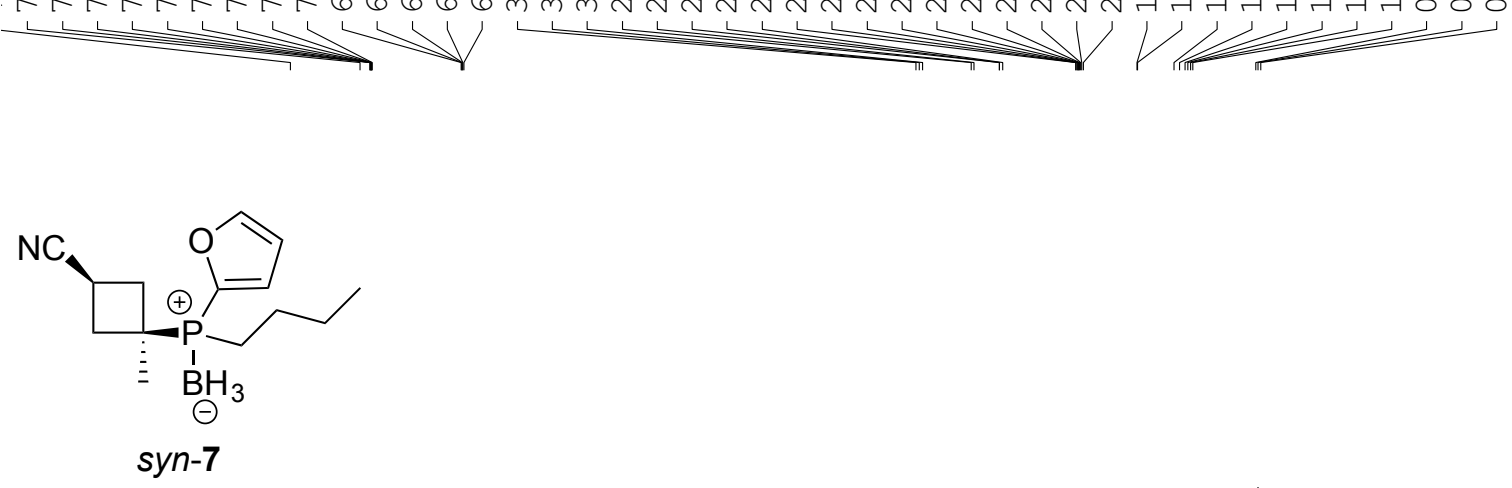
syn isomer
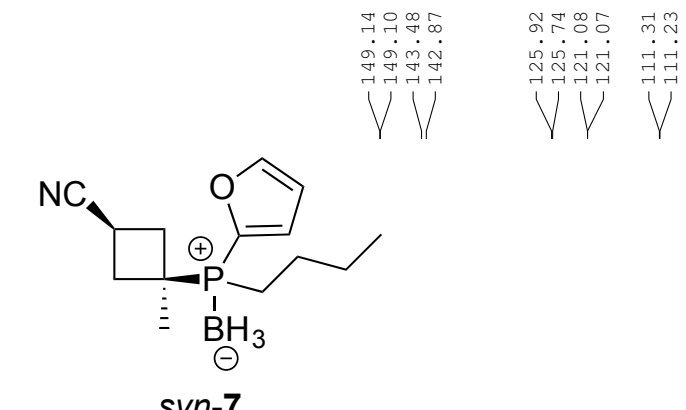

syn-7

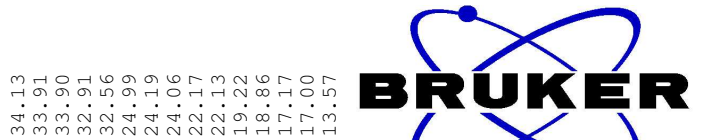

$\overbrace{\text { JAM }-704-32.2}$

EXPNO

PROCNO

Date
Time

PROBHD $5 \mathrm{~mm}$ PADUL $13 \mathrm{C}$

$\begin{array}{lr}\text { PULPROG } & \text { zgpg30 } \\ \text { TD } & 65536\end{array}$

SOLVEN

NS
DS
SWH

FIDRES $\quad 0.366798 \mathrm{~Hz}$

AQ $\quad 1.3631988 \mathrm{sec}$

DW $\quad 20.800$ usec

$3.00000000 \mathrm{sec}$

$0.03000000 \mathrm{sec}$

D11
TD 0

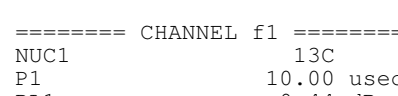

$-0.44 \mathrm{~dB}$

$\begin{array}{ll}\text { PL1W } & 39.19395828 \mathrm{~W} \\ \text { SFO1 } & 100.6479773 \mathrm{MH}\end{array}$

$========$ CHANNEL $\mathrm{f} 2$ $========$

CPDPRG2 waltz16

NUC2

PL2 2

PL13
PL2W

PL12N

$\mathrm{PL} 13 \mathrm{~W}$

$\mathrm{SFO}$

$\mathrm{SI}$
$\mathrm{SF}$
WDW

SSB

GB
PC

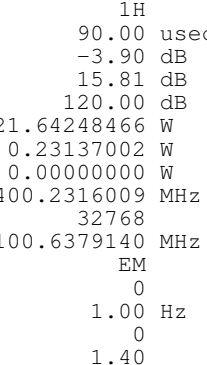

180

160

140

120

100

80

60

40

20

ppm 
syn isomer

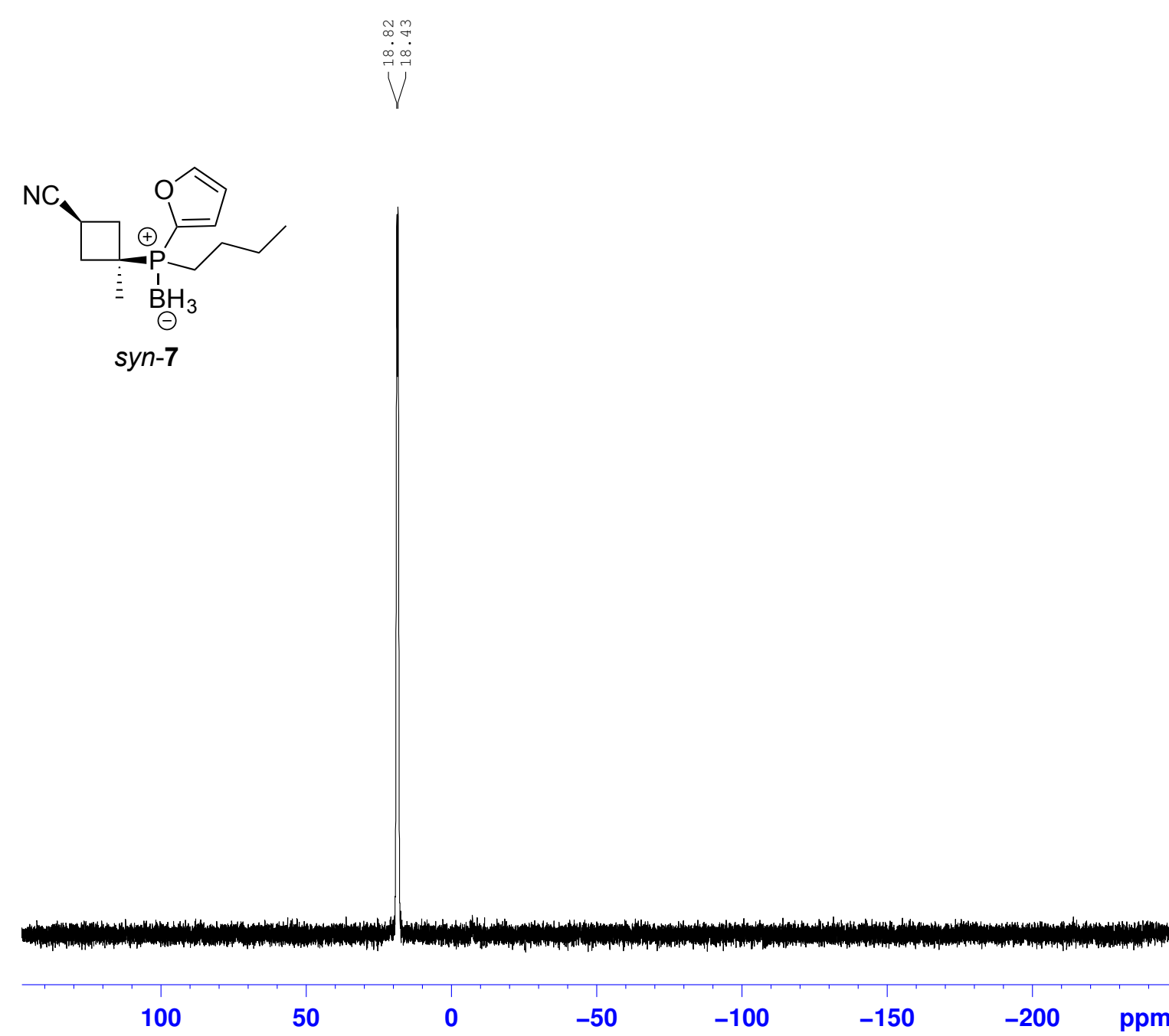

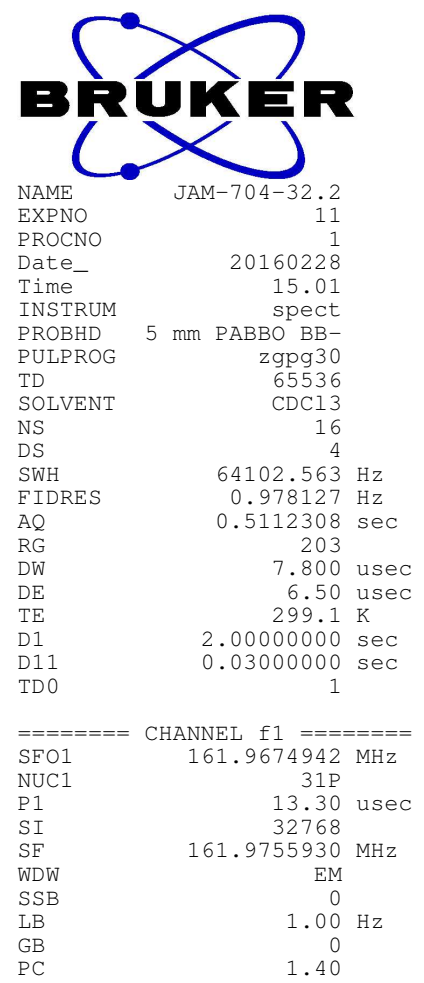$$
\text { pm }
$$ 


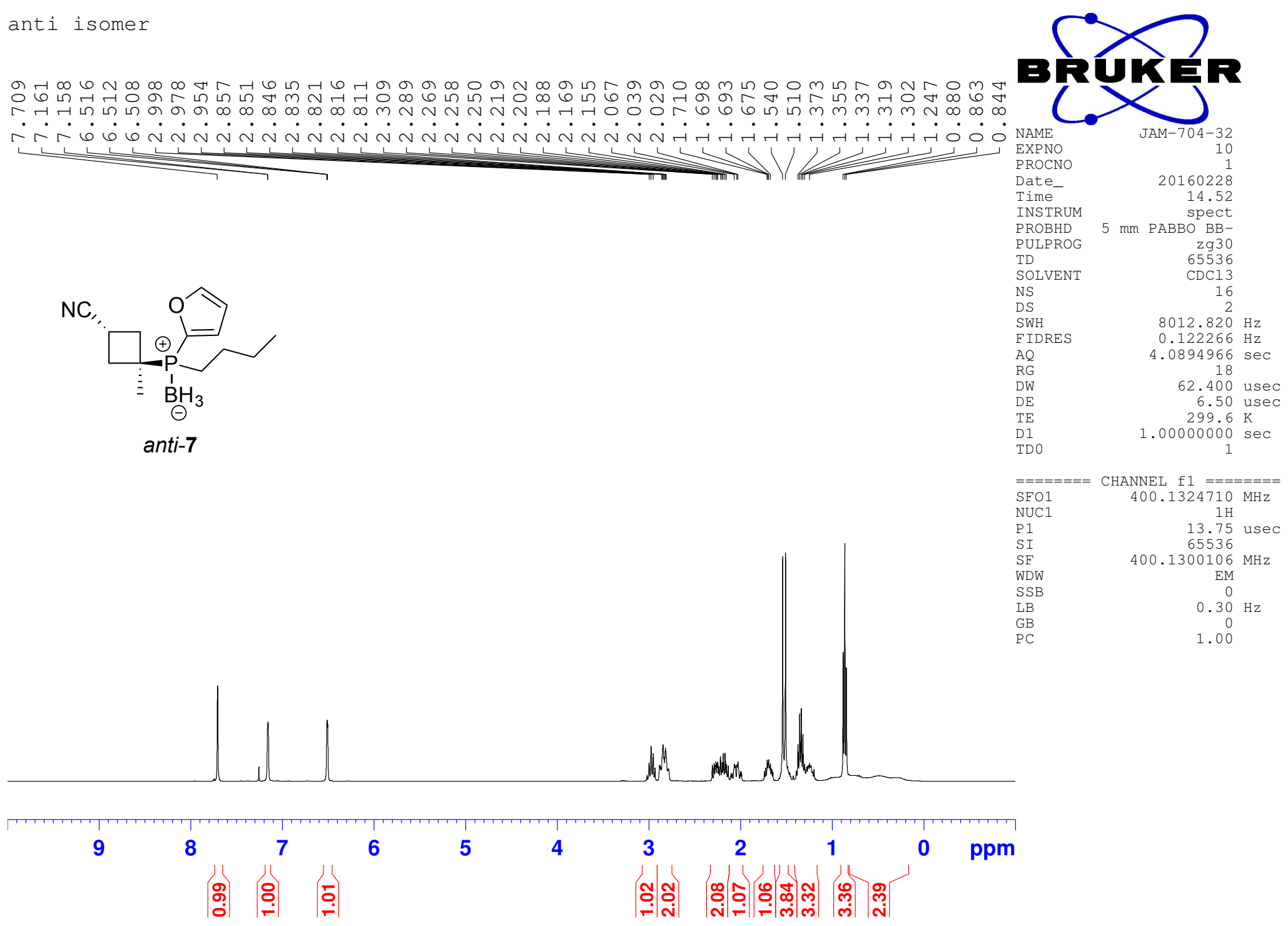


anti isomer

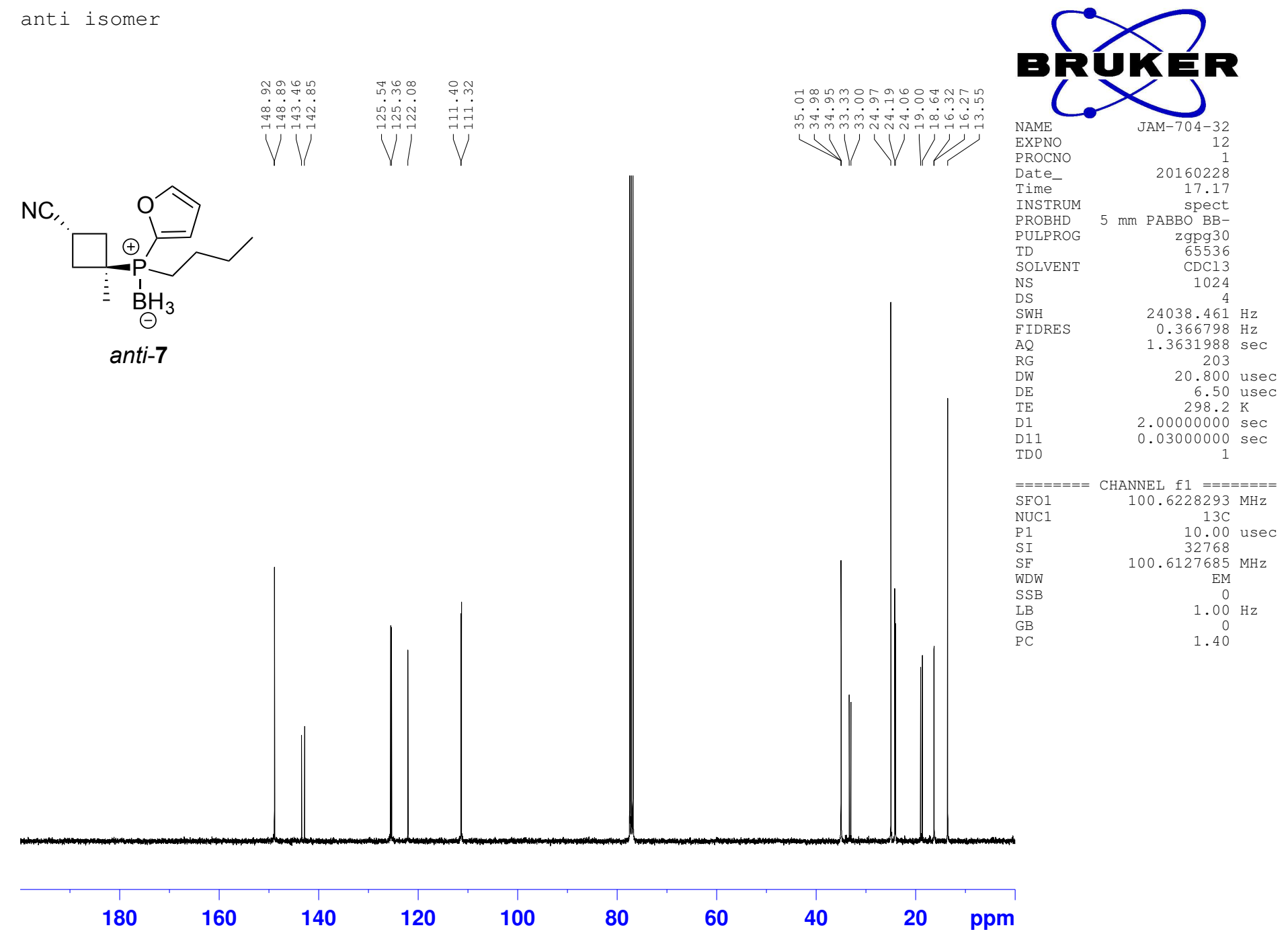


anti isomer
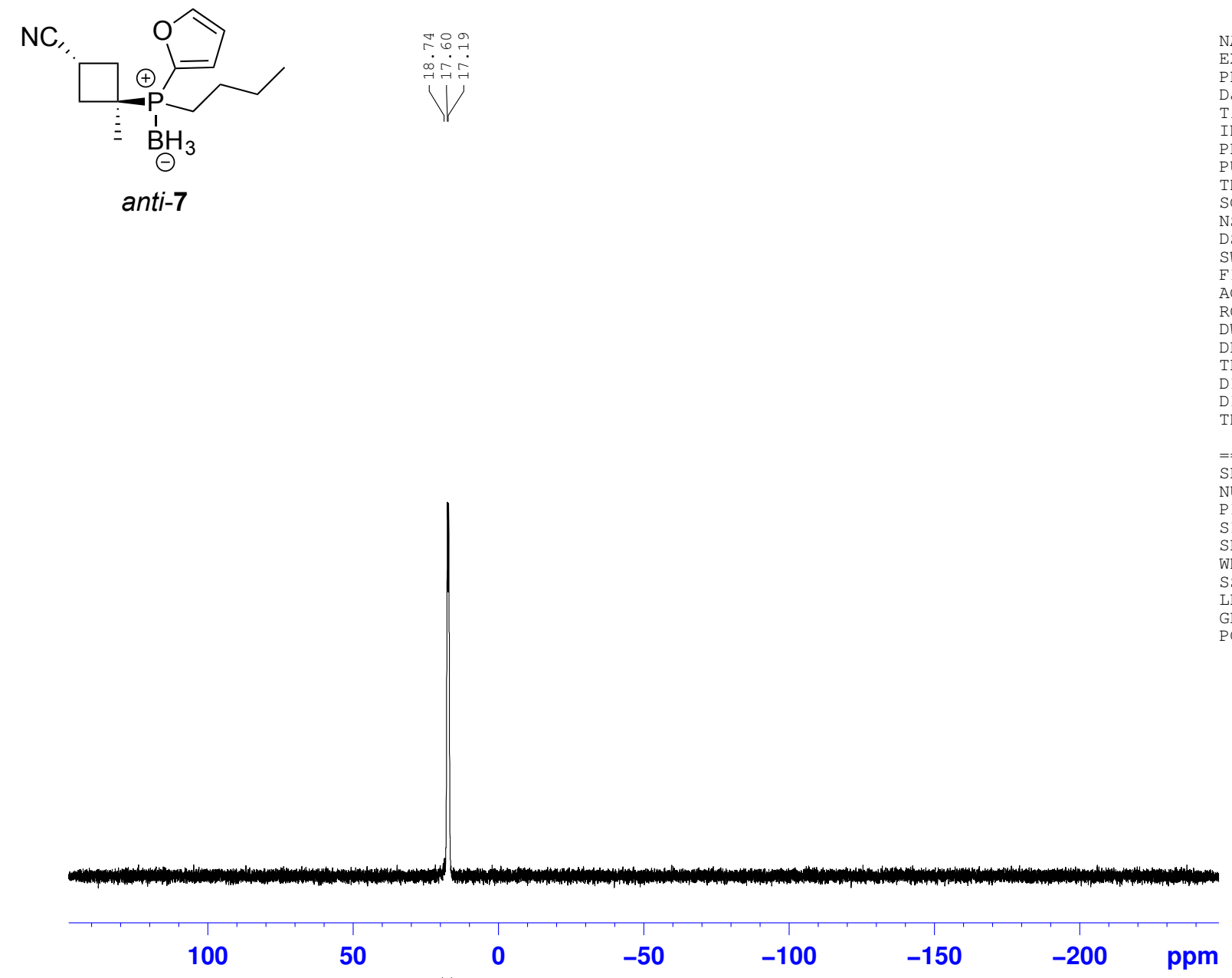

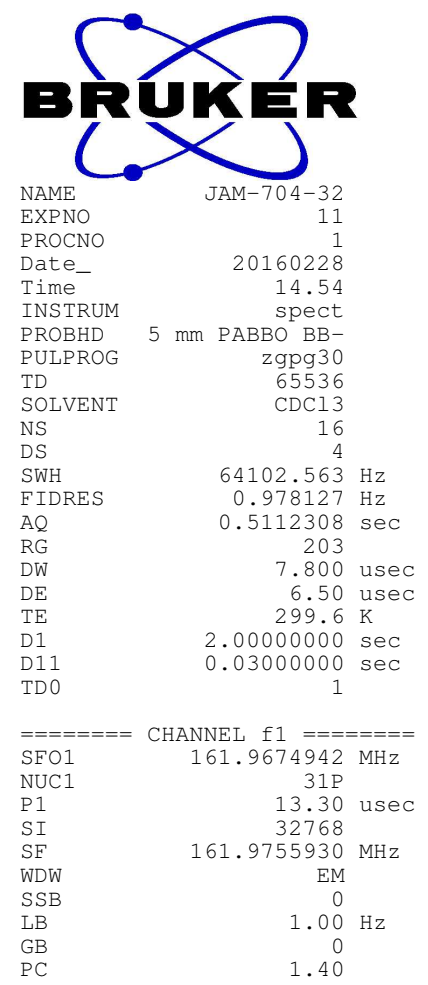

pm 


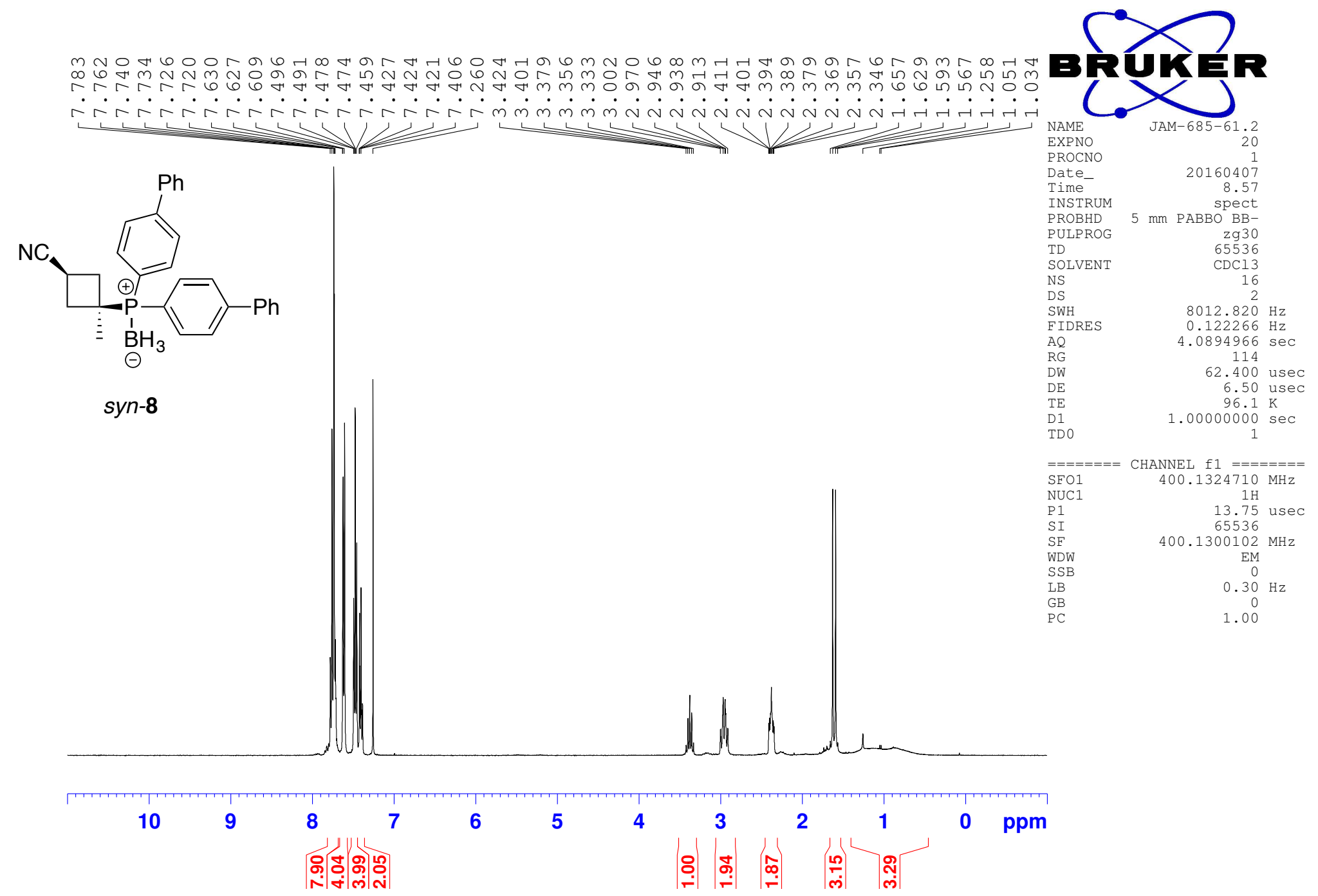




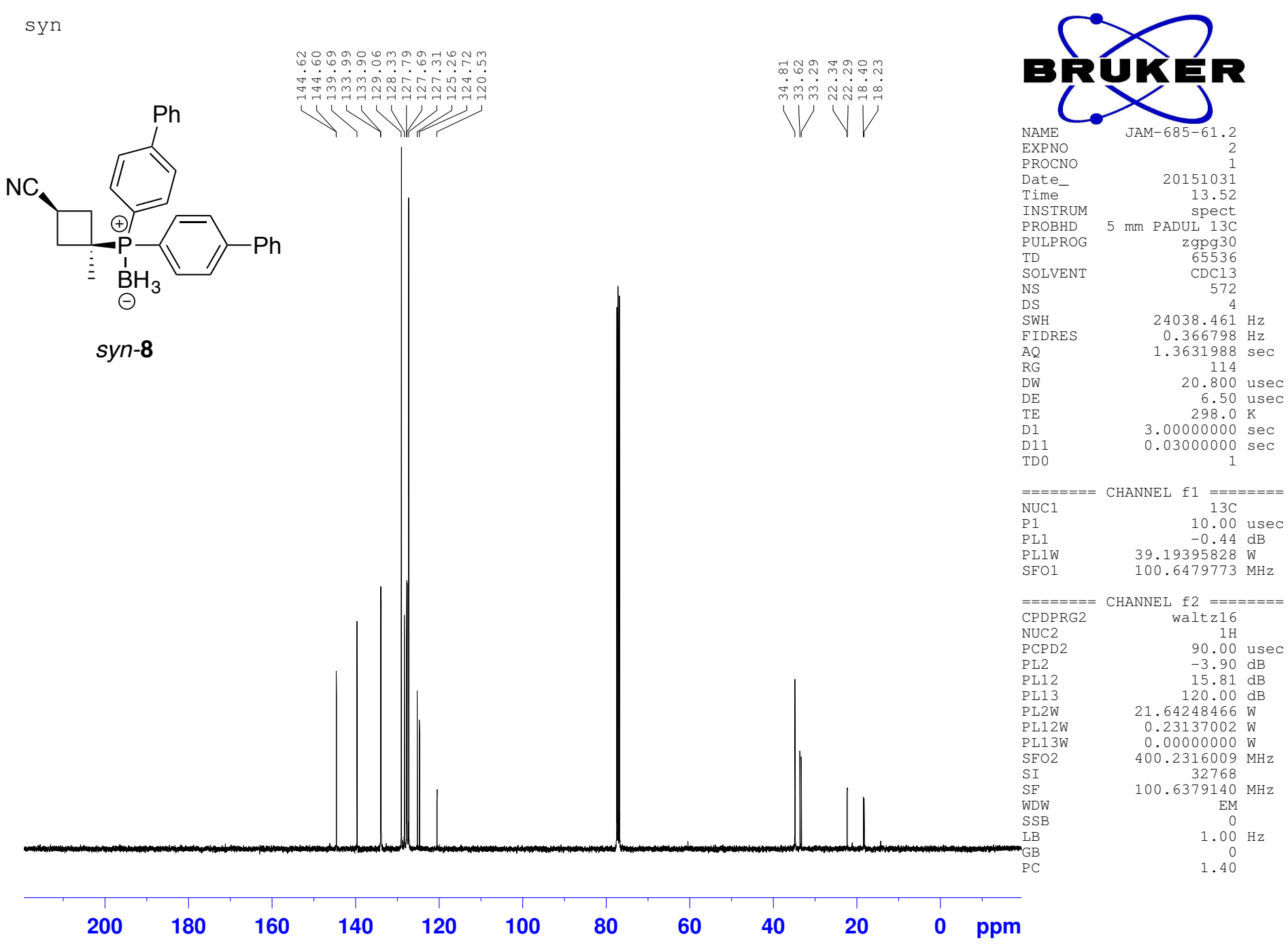




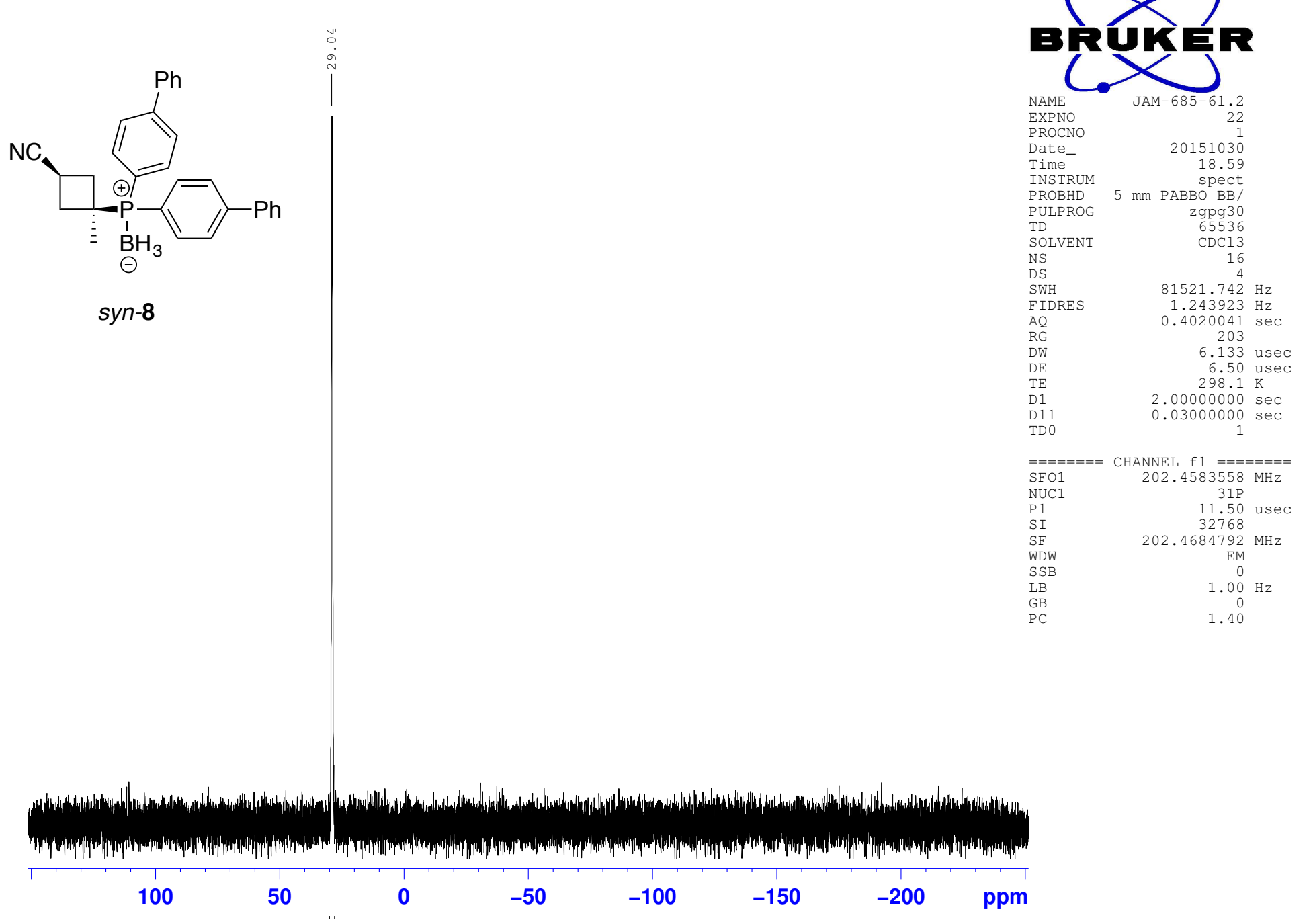




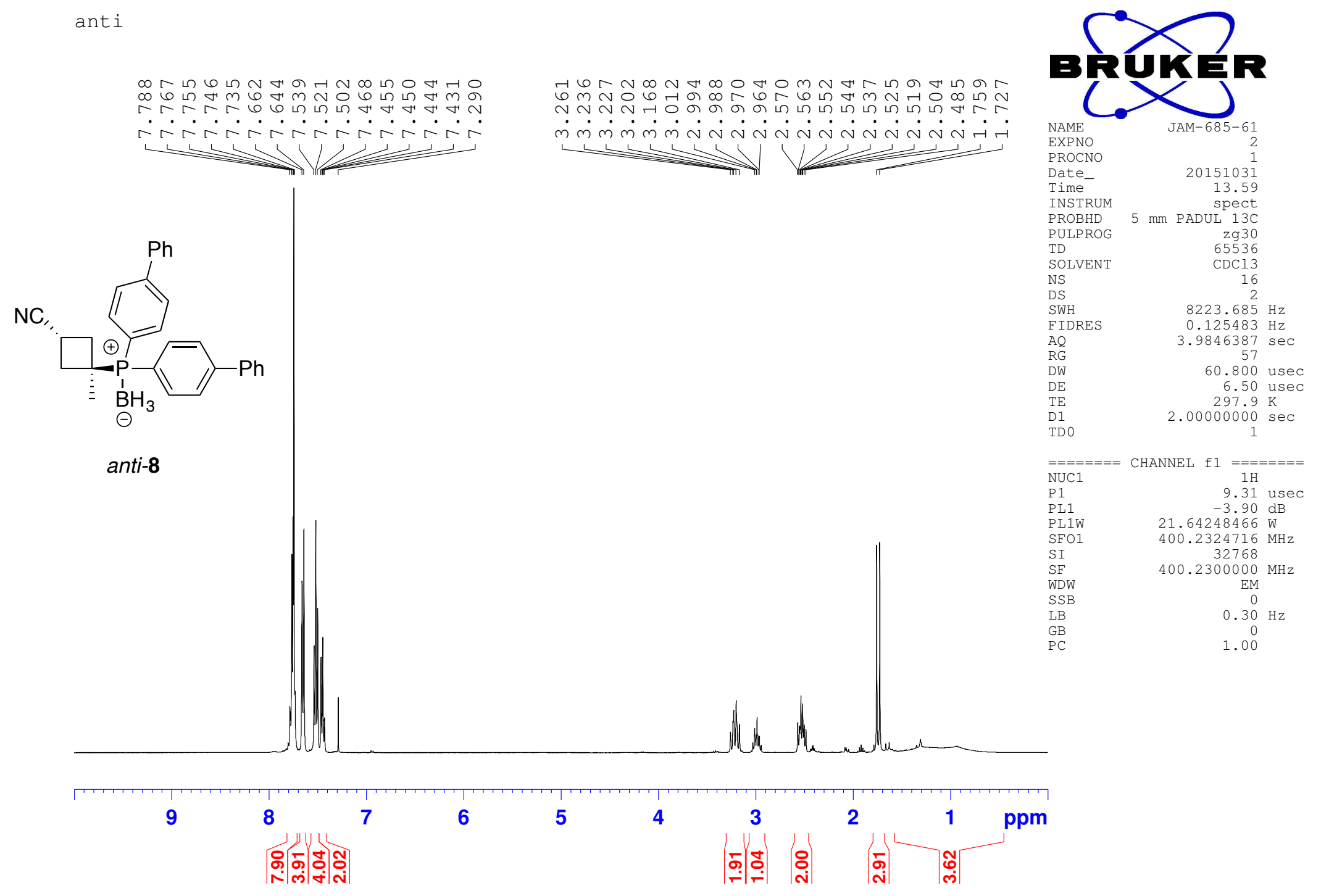


anti

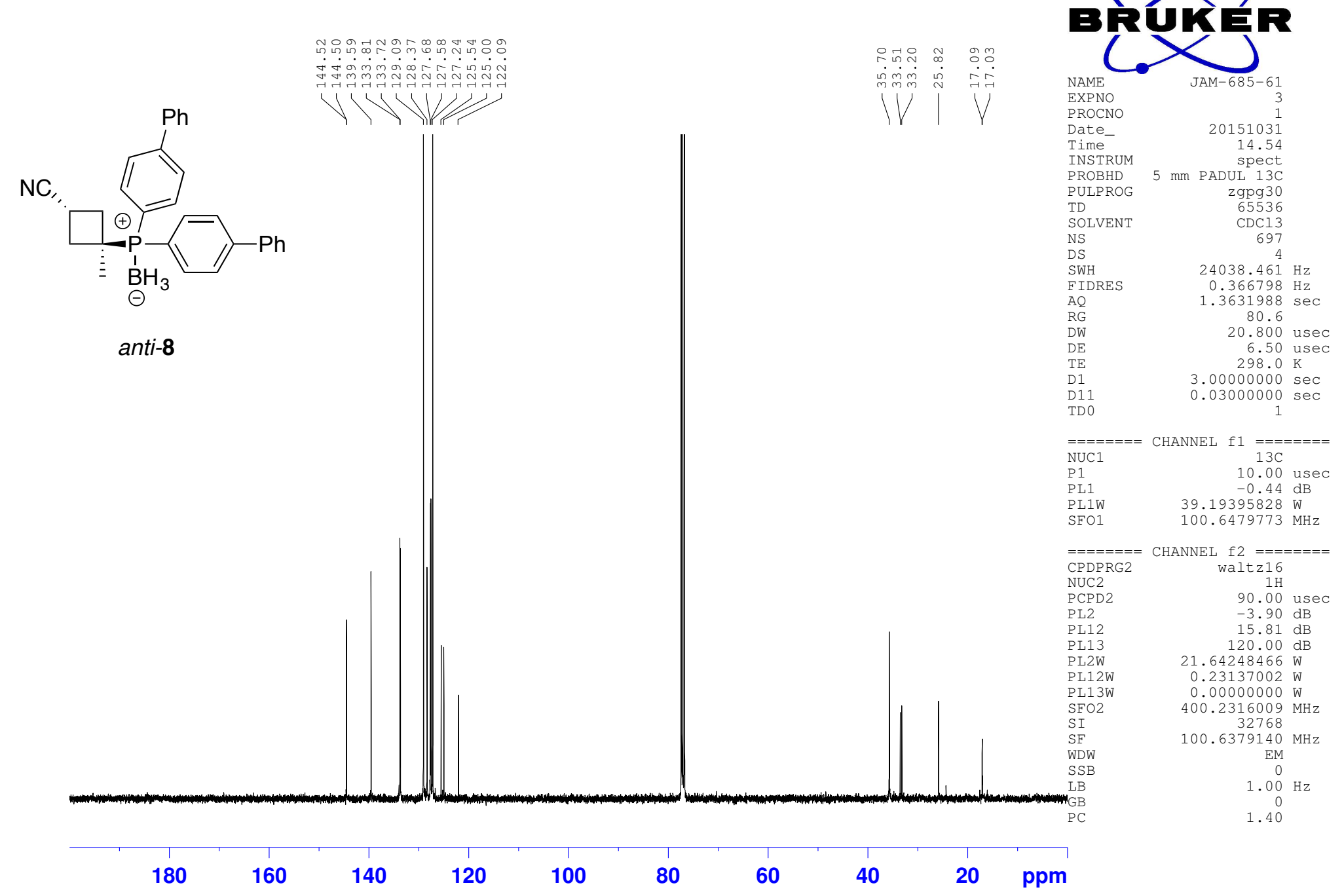




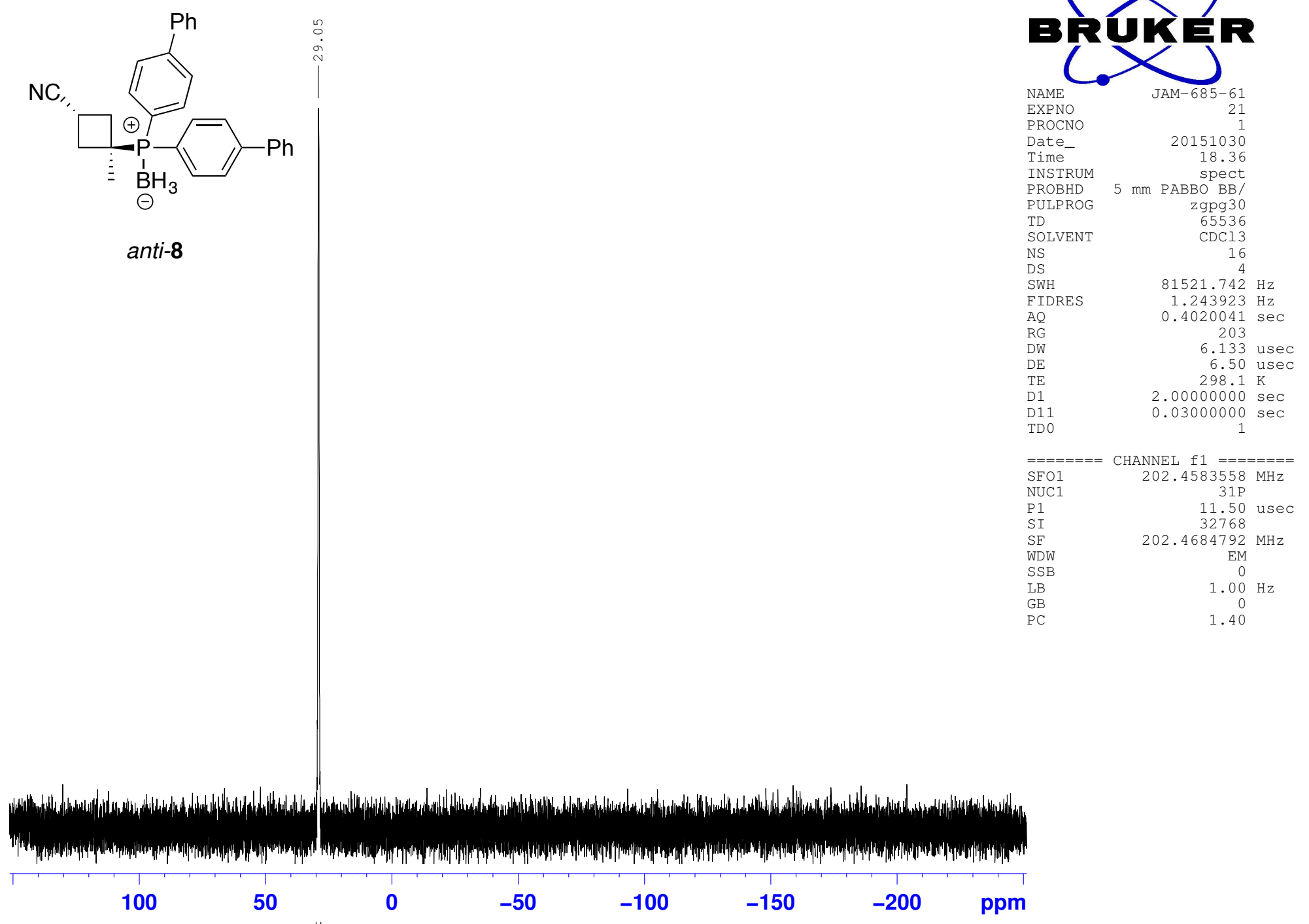




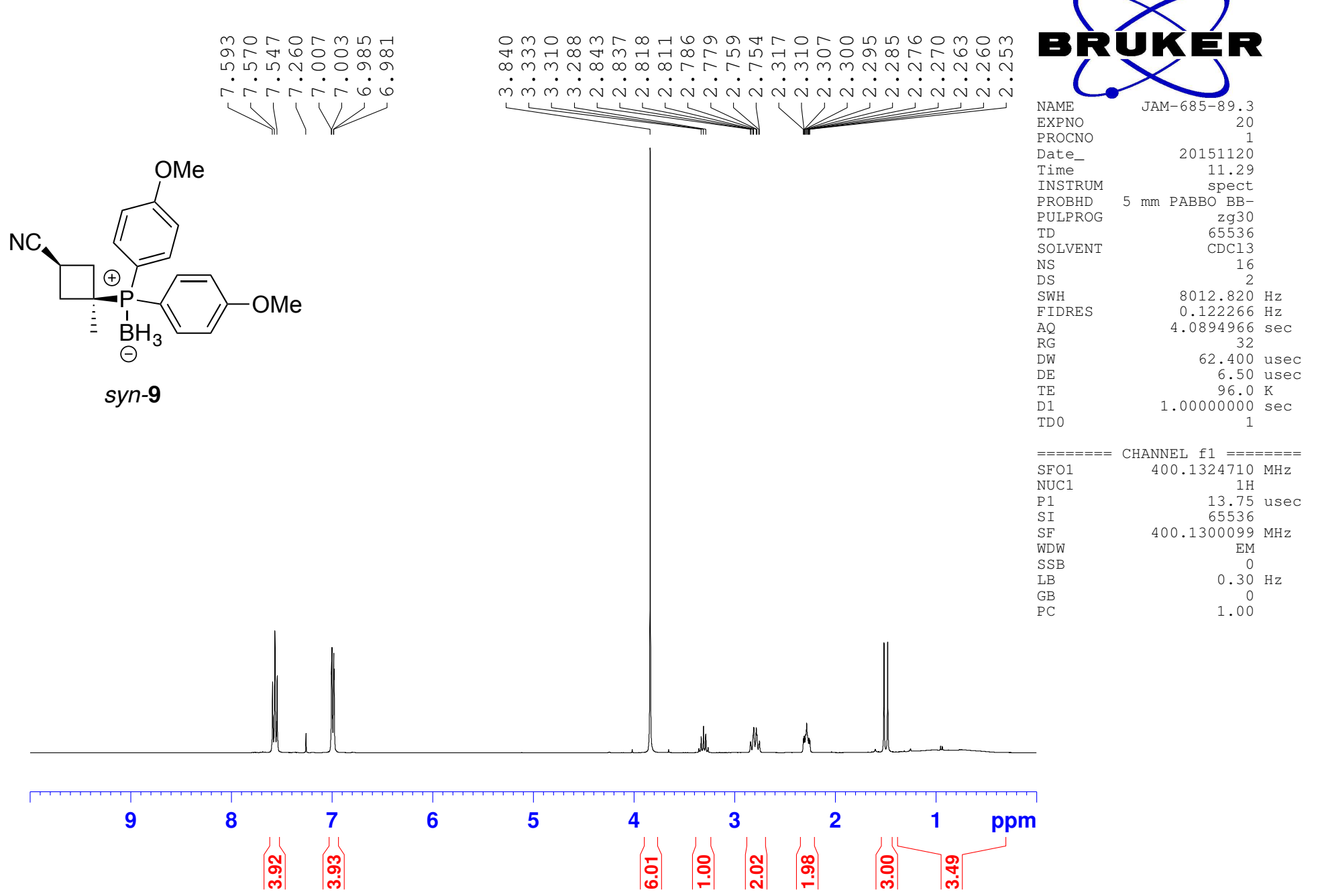




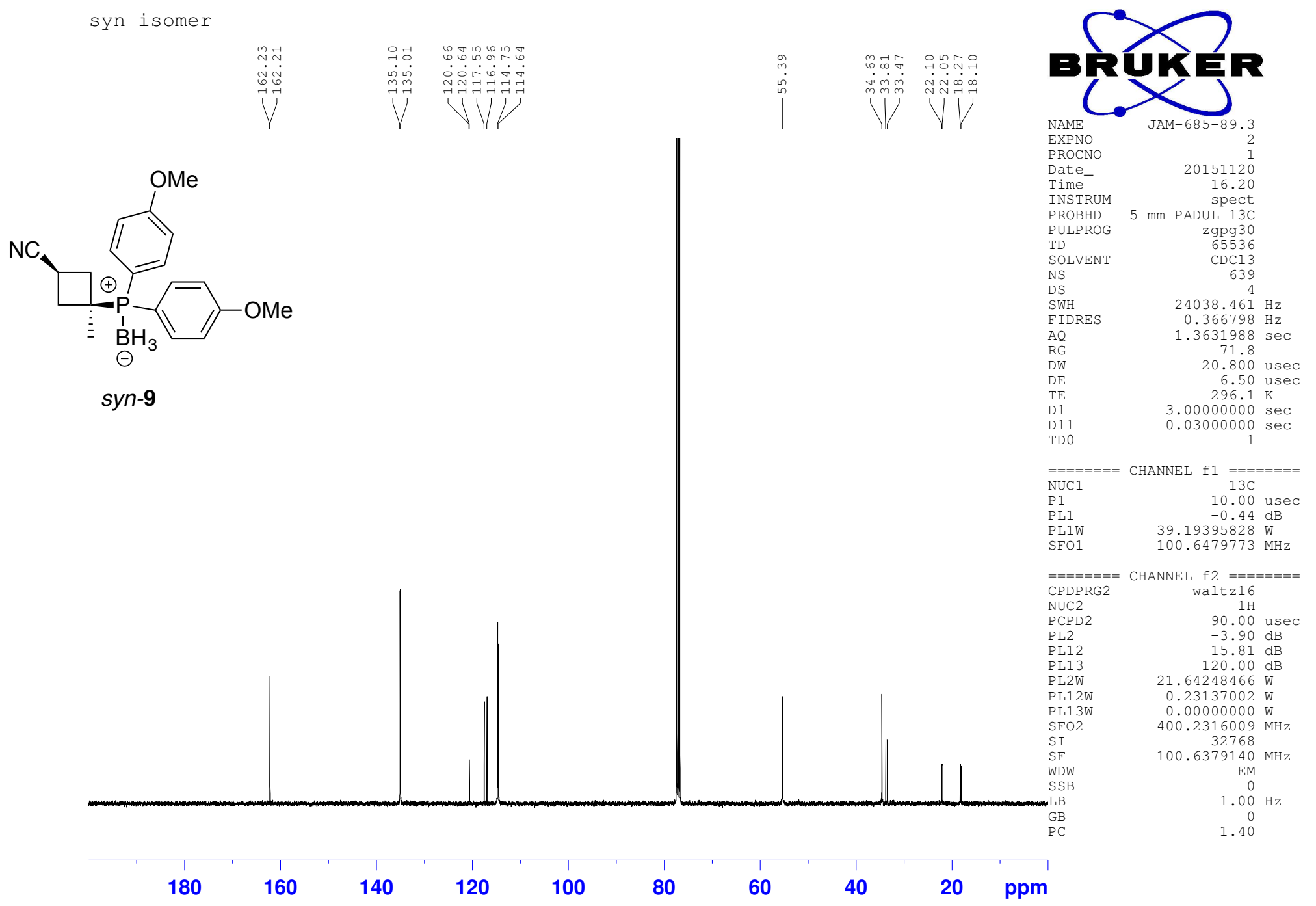




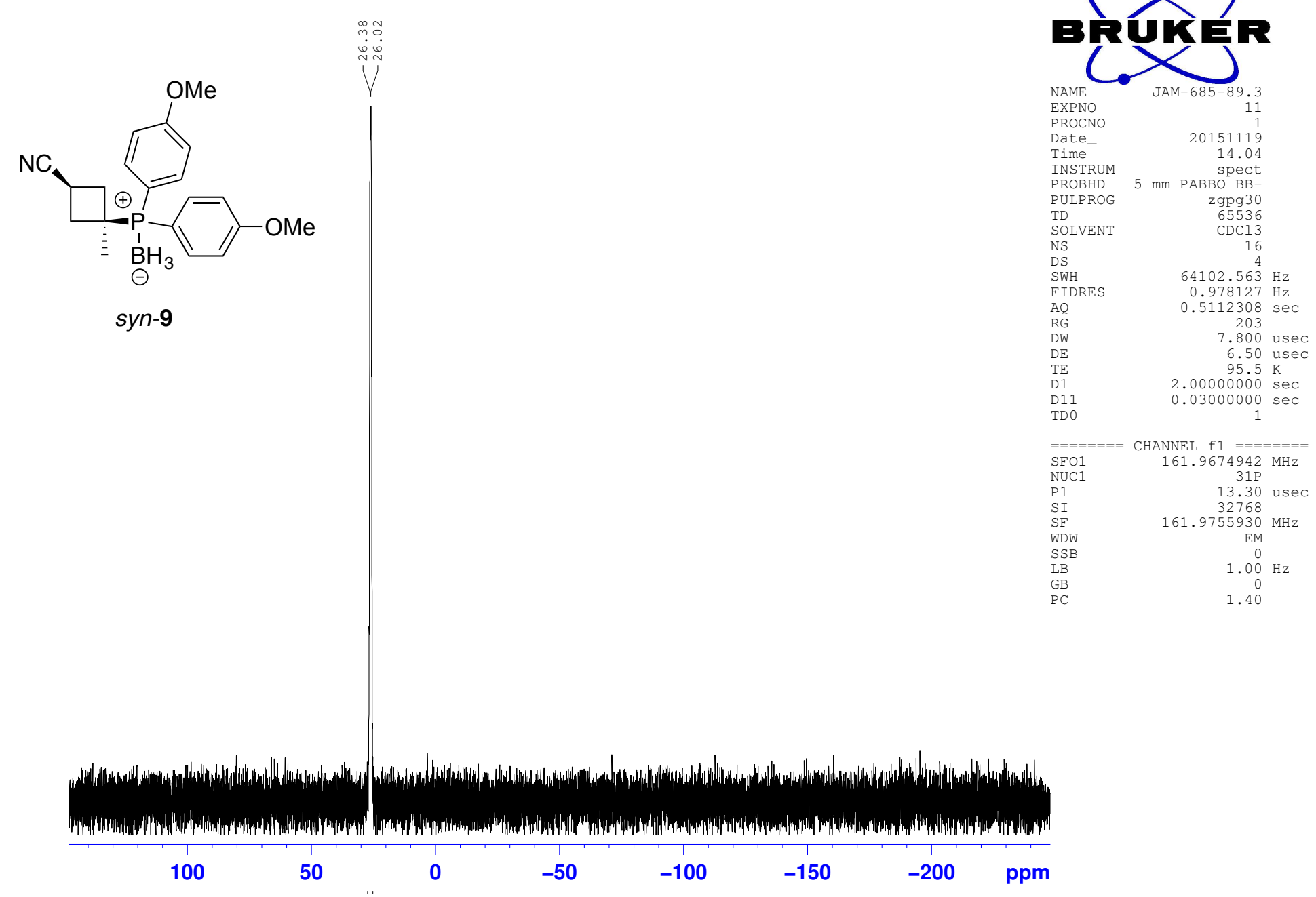


middle spot
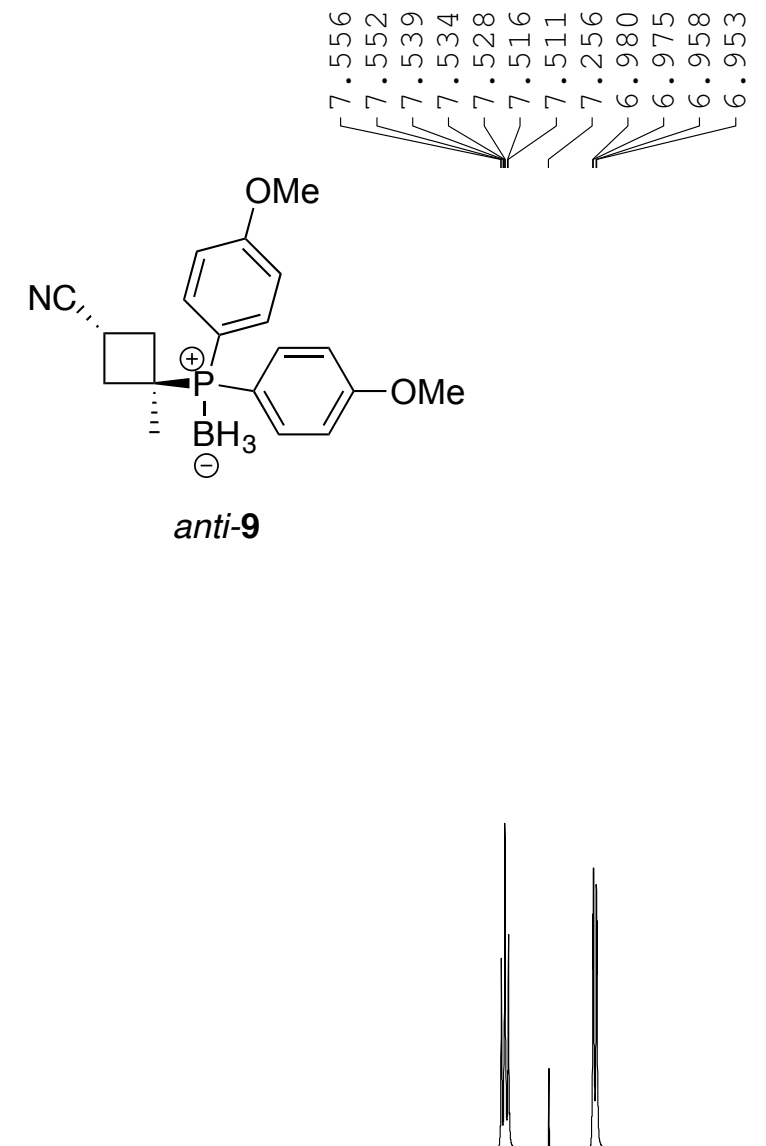

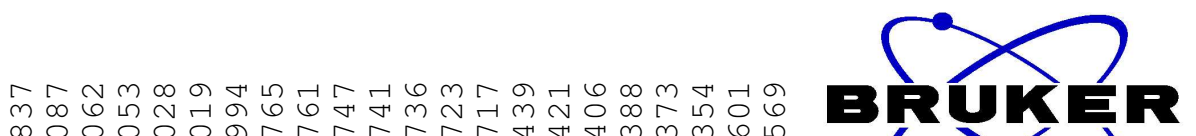

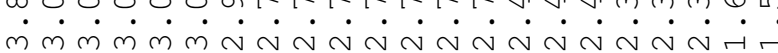
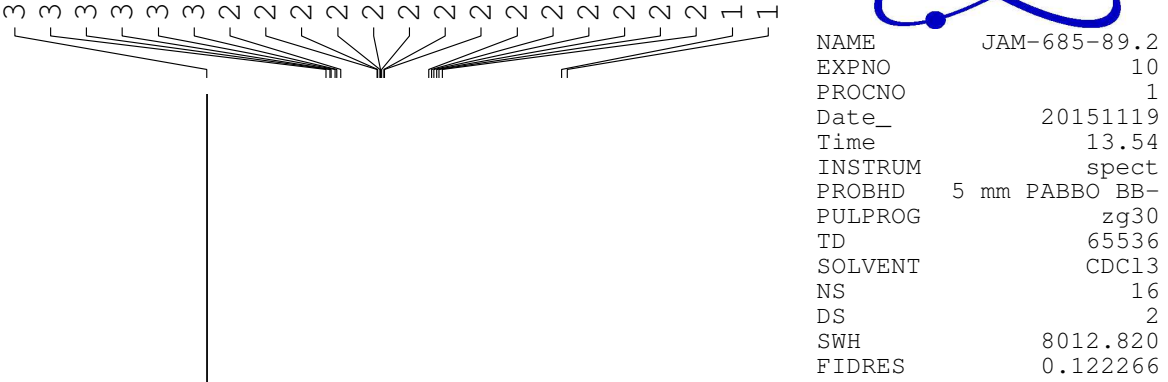

PROCNO

Date_-
Time

$5 \mathrm{~mm}$ PABBO

TD $\quad 65536$

$\begin{array}{lr}\text { CDS } & 16 \\ \text { NS } & 2\end{array}$

SWH $\quad 8012.820 \mathrm{~Hz}$

$\begin{array}{lll}\text { FIDRES } & 0.122266 \mathrm{~Hz} \\ \mathrm{AQ} & 4.0894966 \mathrm{sec}\end{array}$

$\begin{array}{lr}\mathrm{AQ} & 4.0894966 \mathrm{sec} \\ \mathrm{RG} & 57\end{array}$

DW 62.400 usec

.400 usec
6.50 usec $95.3 \mathrm{~K}$

$1.00000000 \mathrm{sec}$

TD 0

$=======$ CHANNEL $\mathrm{f} 1 \mathrm{=}=======$
SFO1
$400.1324710 \mathrm{MHz}$

$\begin{array}{lrl}\text { SFO1 } & 400.1324710 \mathrm{MHz} \\ \text { NUC1 } & 1 \mathrm{H}\end{array}$

$\begin{array}{lr}\text { NUC1 } & 1 \mathrm{H} \\ \mathrm{P1} & 13.75 \mathrm{usec}\end{array}$

SF $\quad 400.1300121 \mathrm{MHz}$

$\begin{array}{cc}\text { WDW } & \text { EM } \\ \text { SSB } & 0\end{array}$

SSB
LB

LB

0
$0.30 \mathrm{~Hz}$

0
1.00

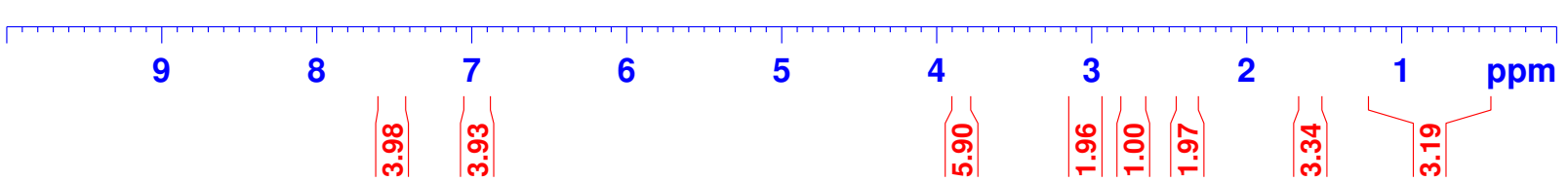


middle spot

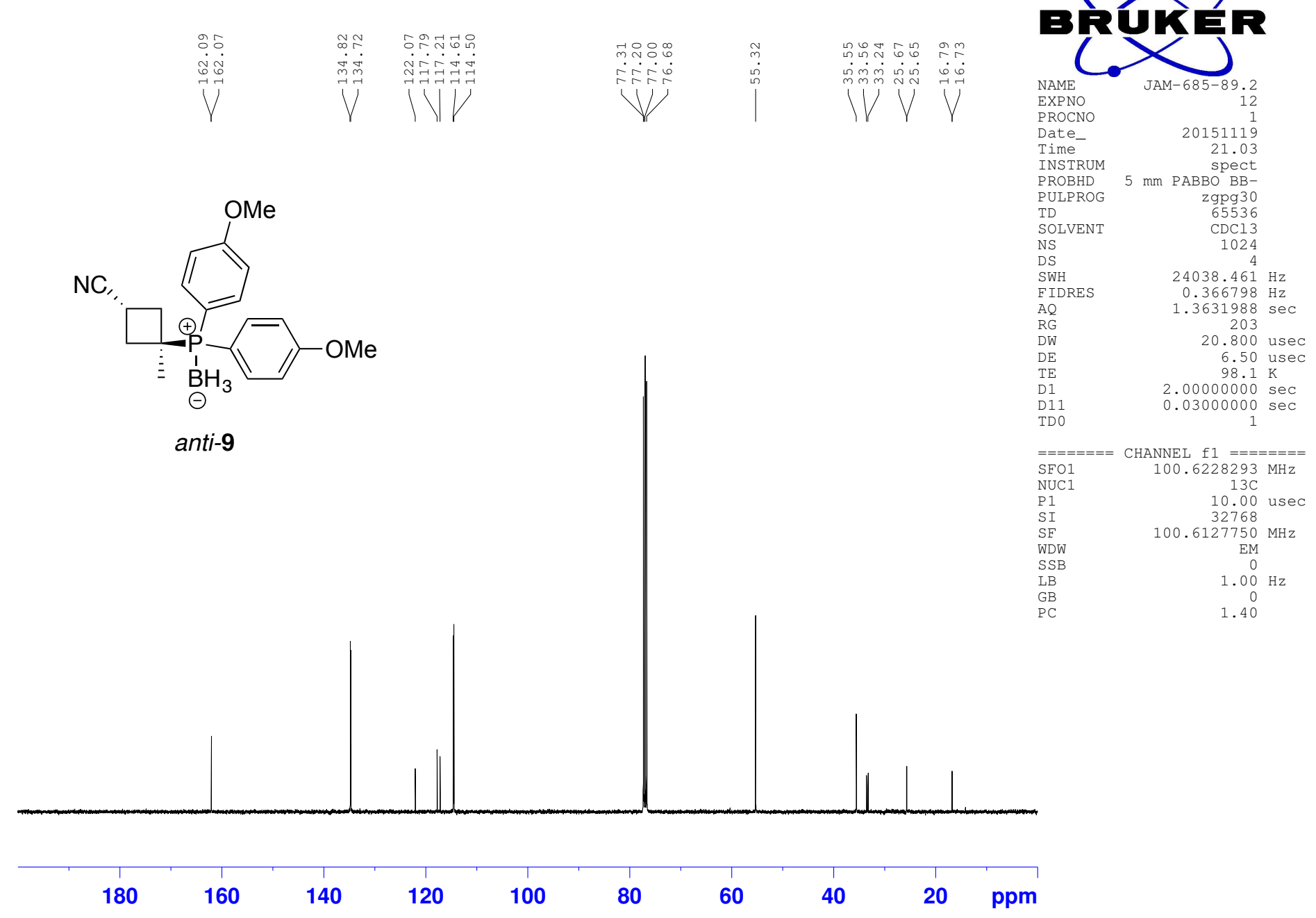


middle spot

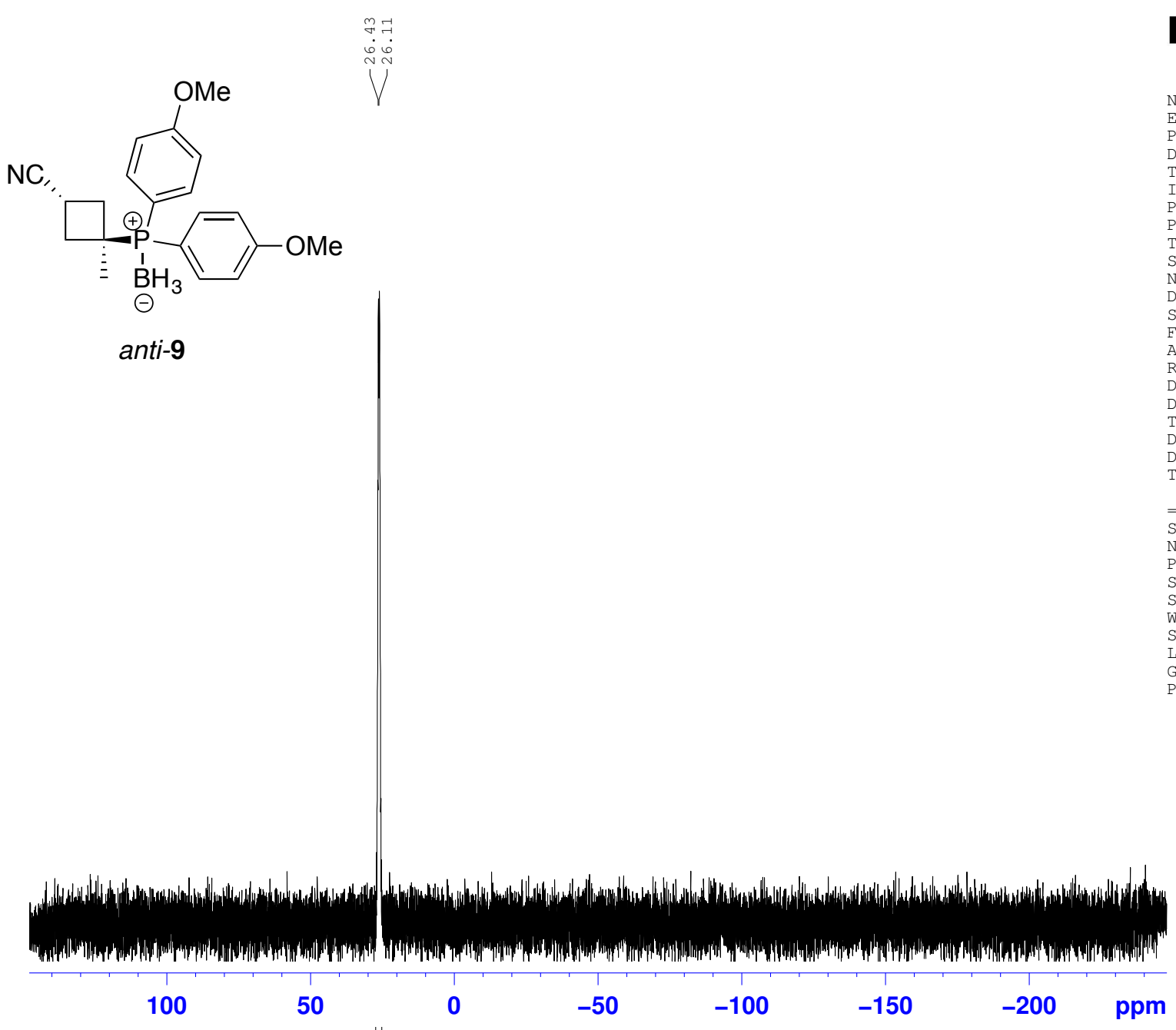

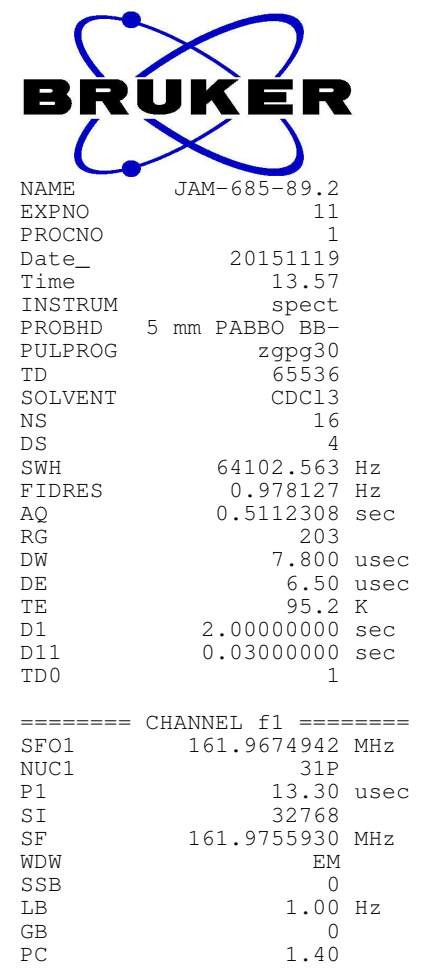

ppm 


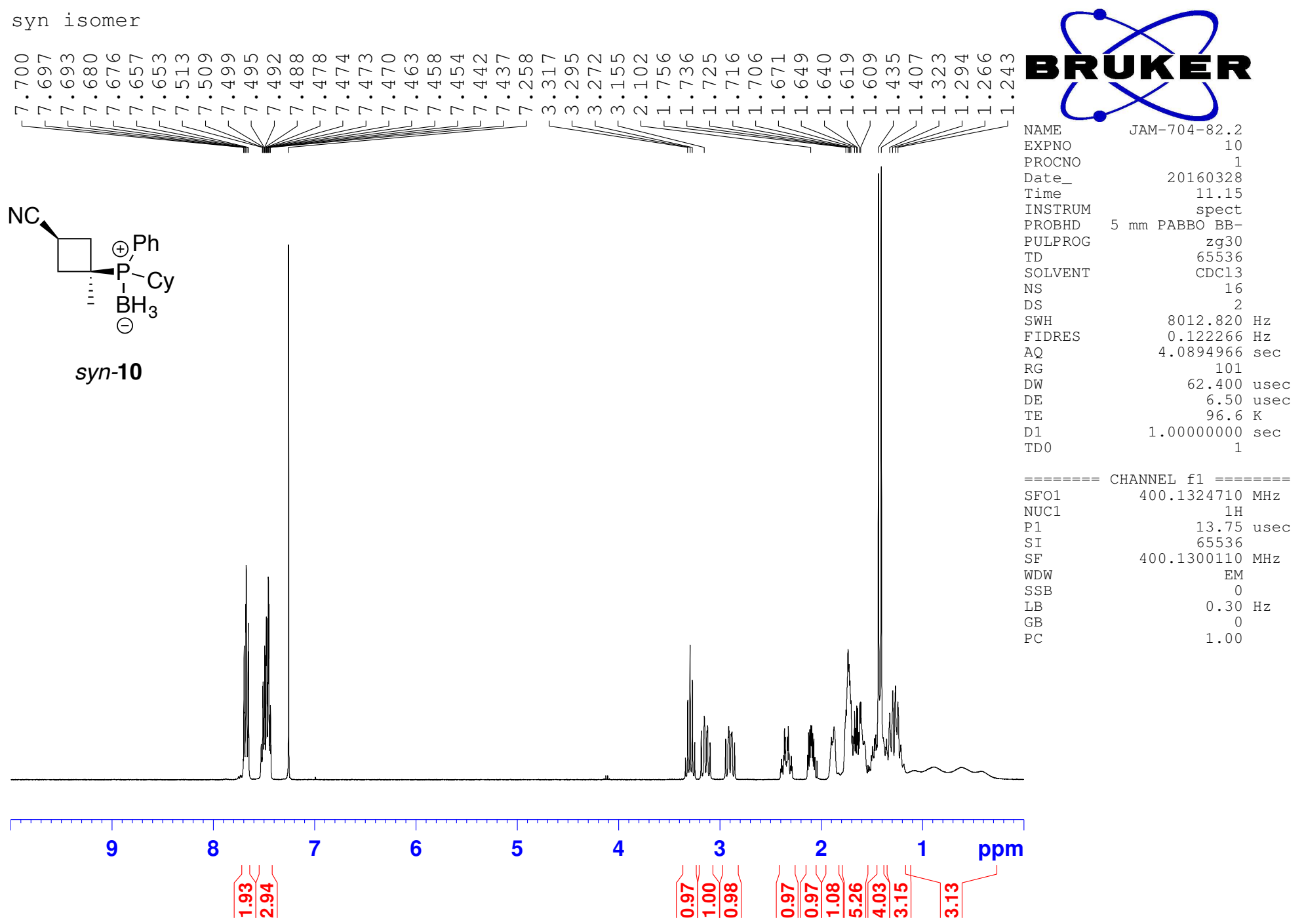


syn isomer

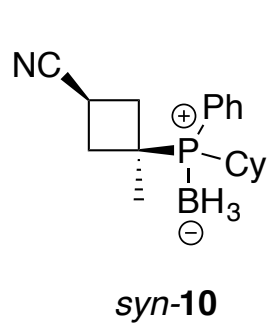

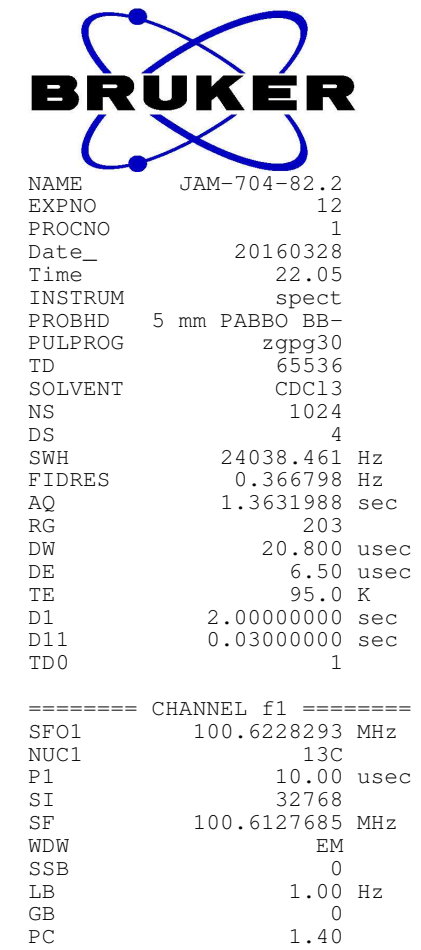

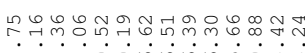
im
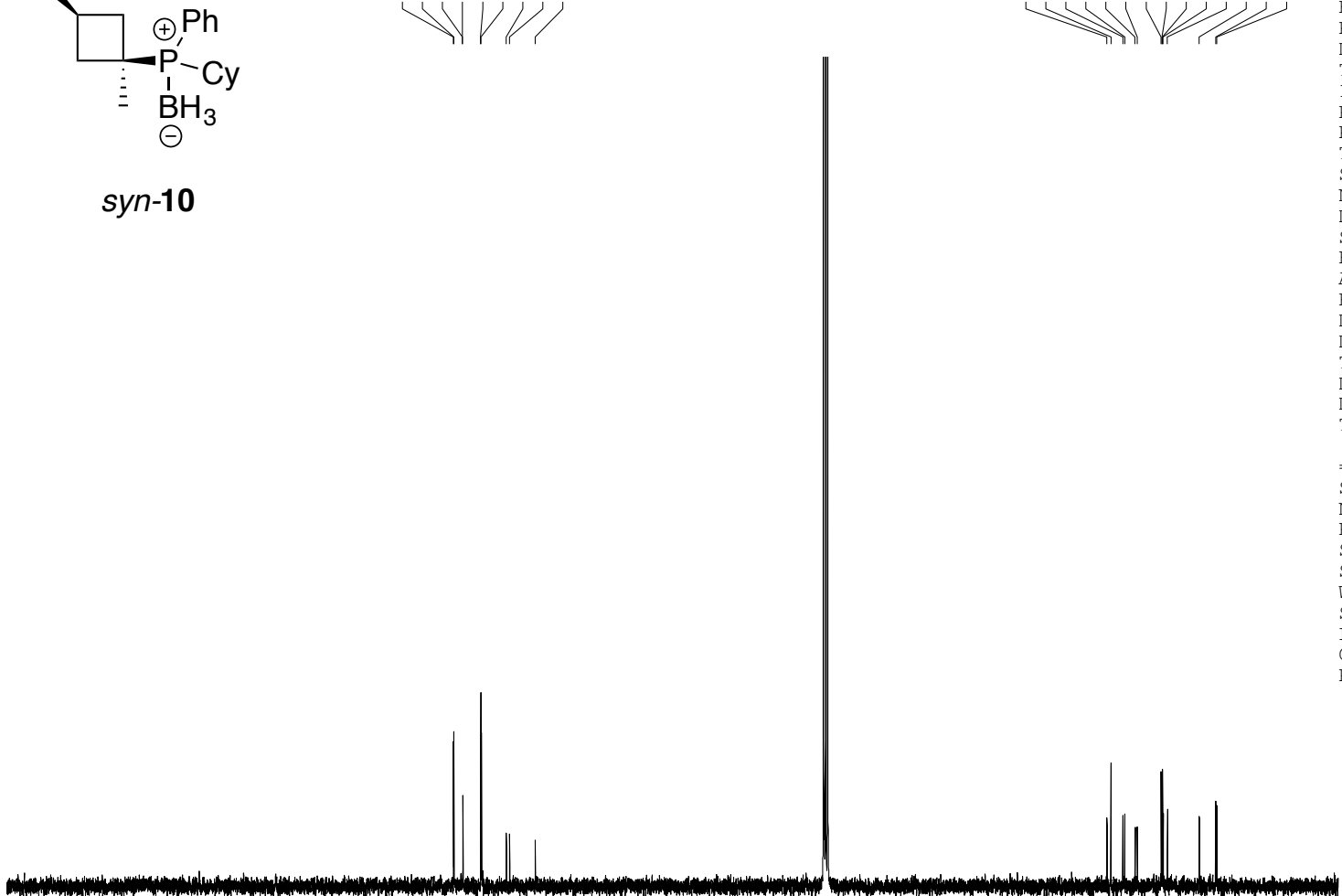

(n)

$\begin{array}{llllllllll}80 & 160 & 140 & 120 & 100 & 80 & 60 & 40 & 20 & \text { ppm }\end{array}$


syn isomer
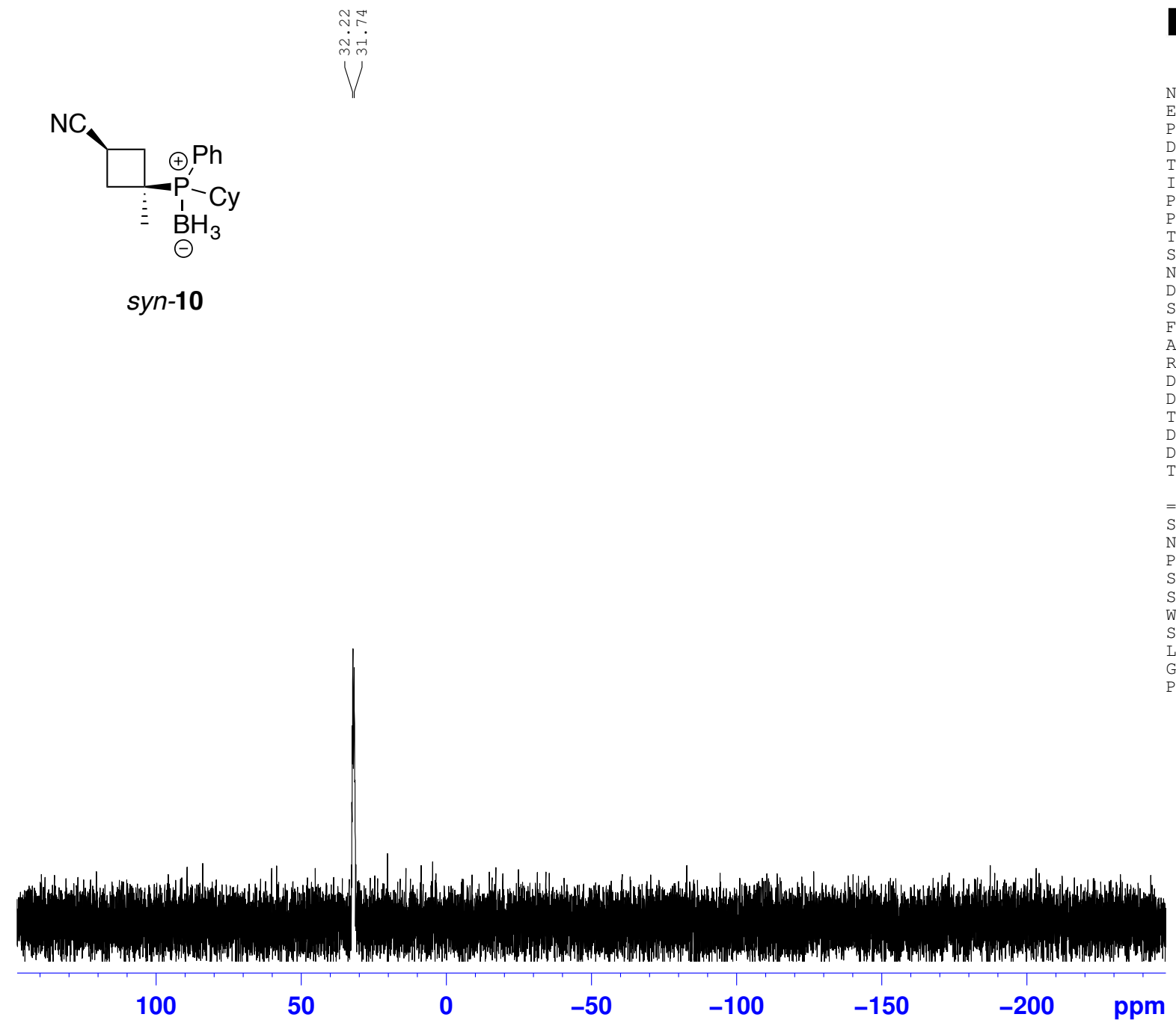

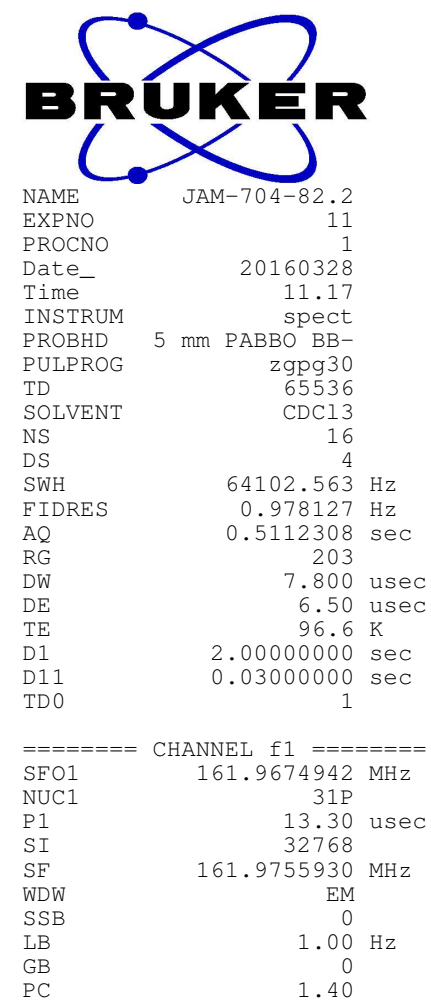

pm 


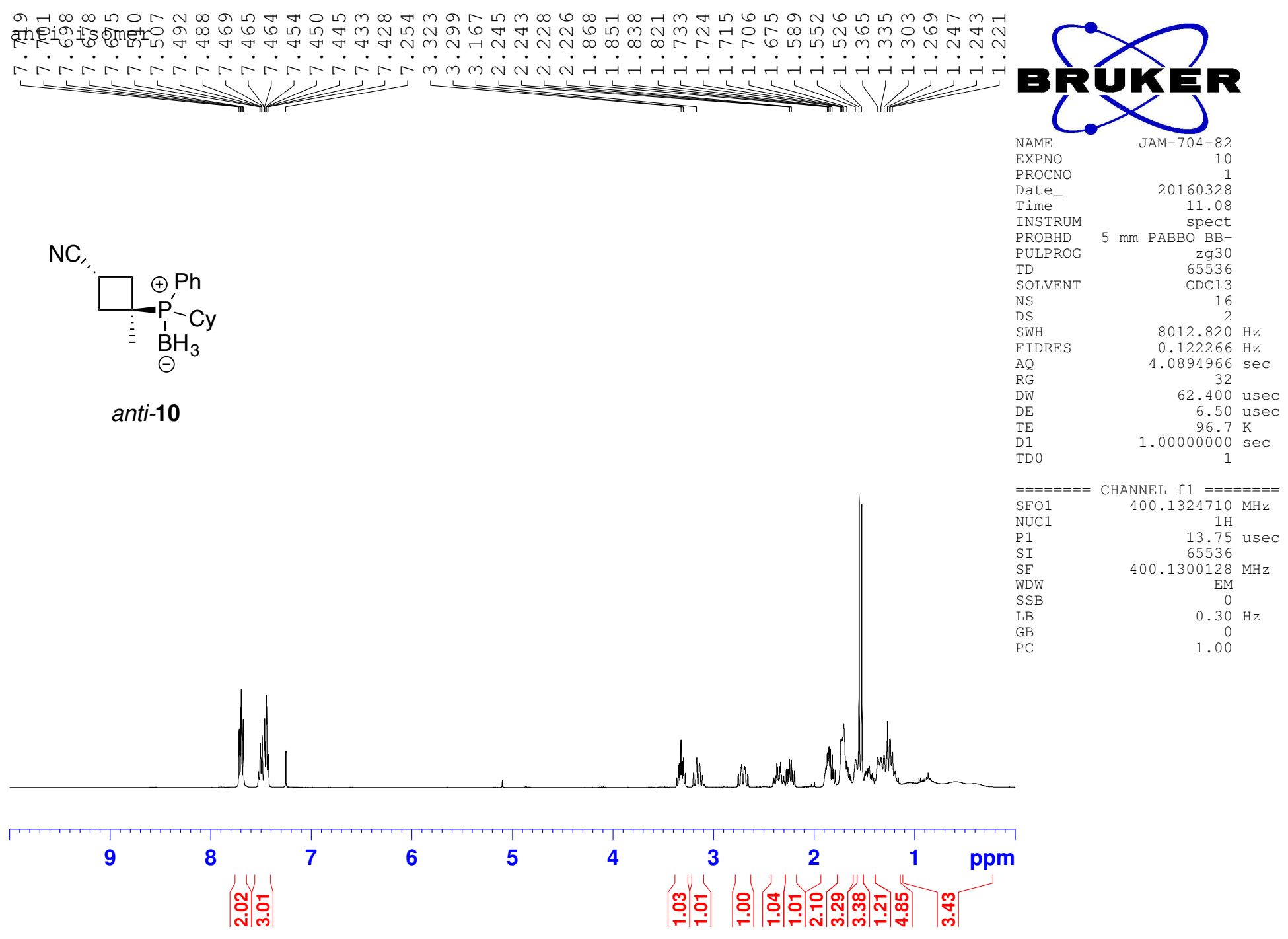


anti isomer

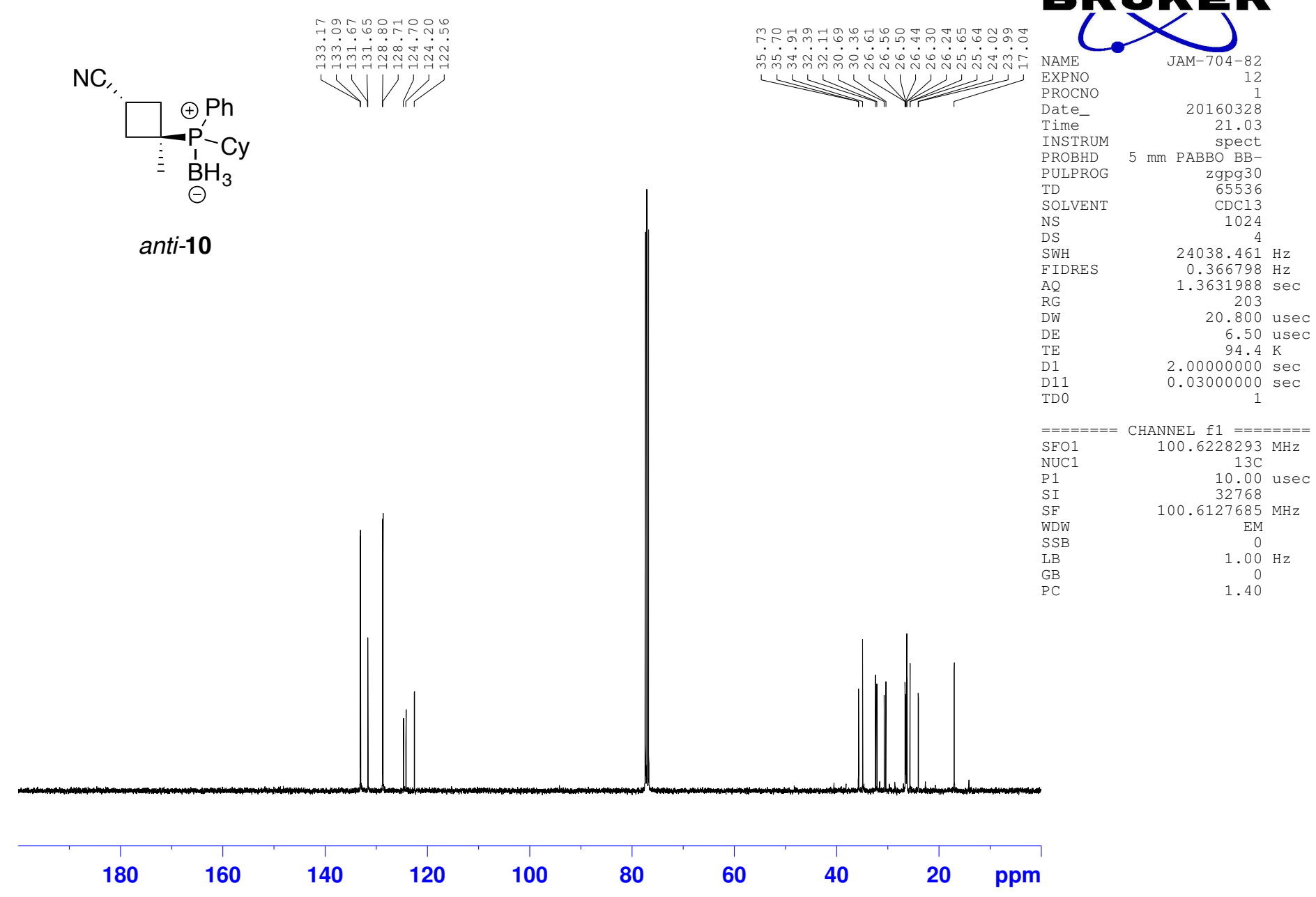




\section{anti isomer}

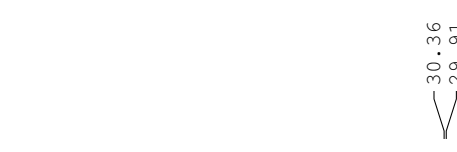

NC,

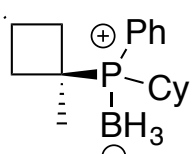

anti-10

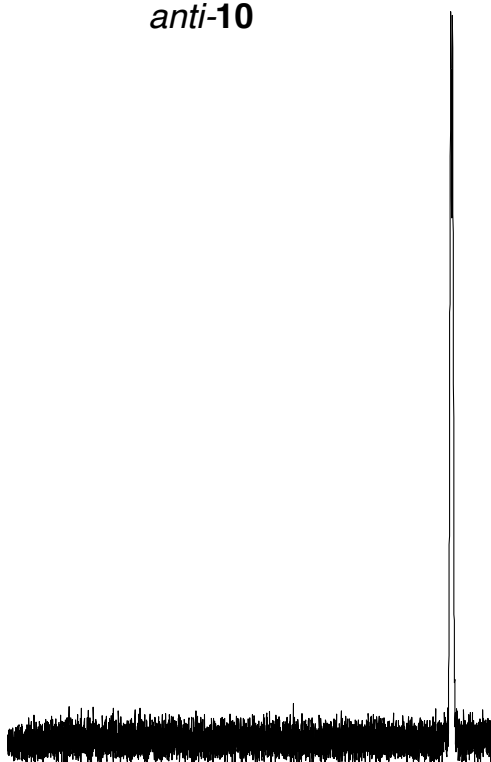

\section{Gruker}

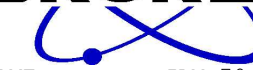

EXPNO

PROCNO

Time-

INSTRUM
PROBHD

PULPRO

TD

DS

SWH

$\mathrm{AQ}$

RG

DW

TE
D1
D11

D 11
TD 0

$=======$ CHANNEL $\mathrm{fl}=======$

$=======$ CHANNEL $\mathrm{f} 1 \mathrm{I}=======$
SFO1 $\quad 161.9674942 \mathrm{MHz}$

NUC1 $\quad 31 \mathrm{P}$

P1

SF
WDW
SSB

SSB
LB
GB

$161.9755930 \mathrm{MHz}$

$\mathrm{EM}$
0

$1.00 \mathrm{~Hz}$

0
1.40 


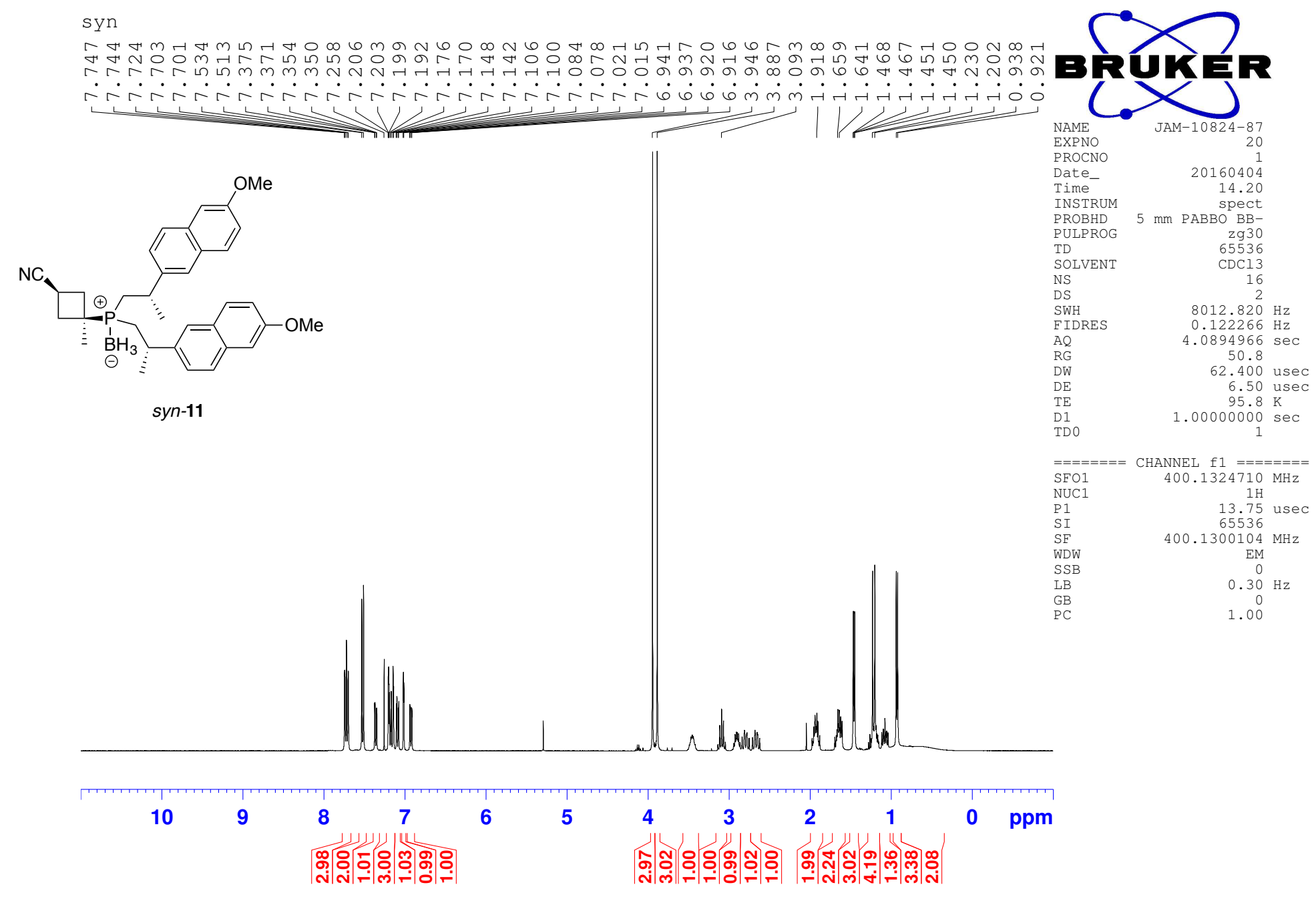




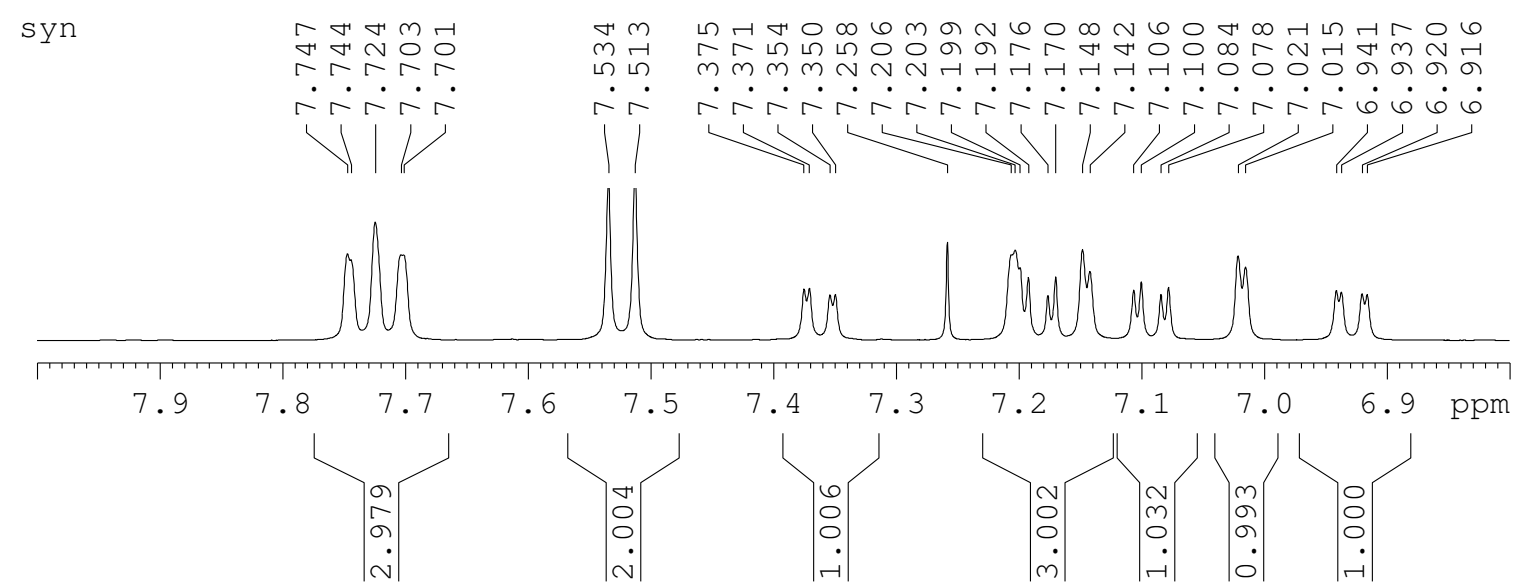

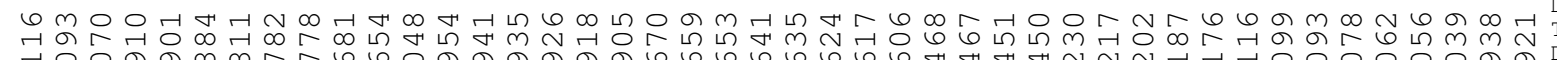
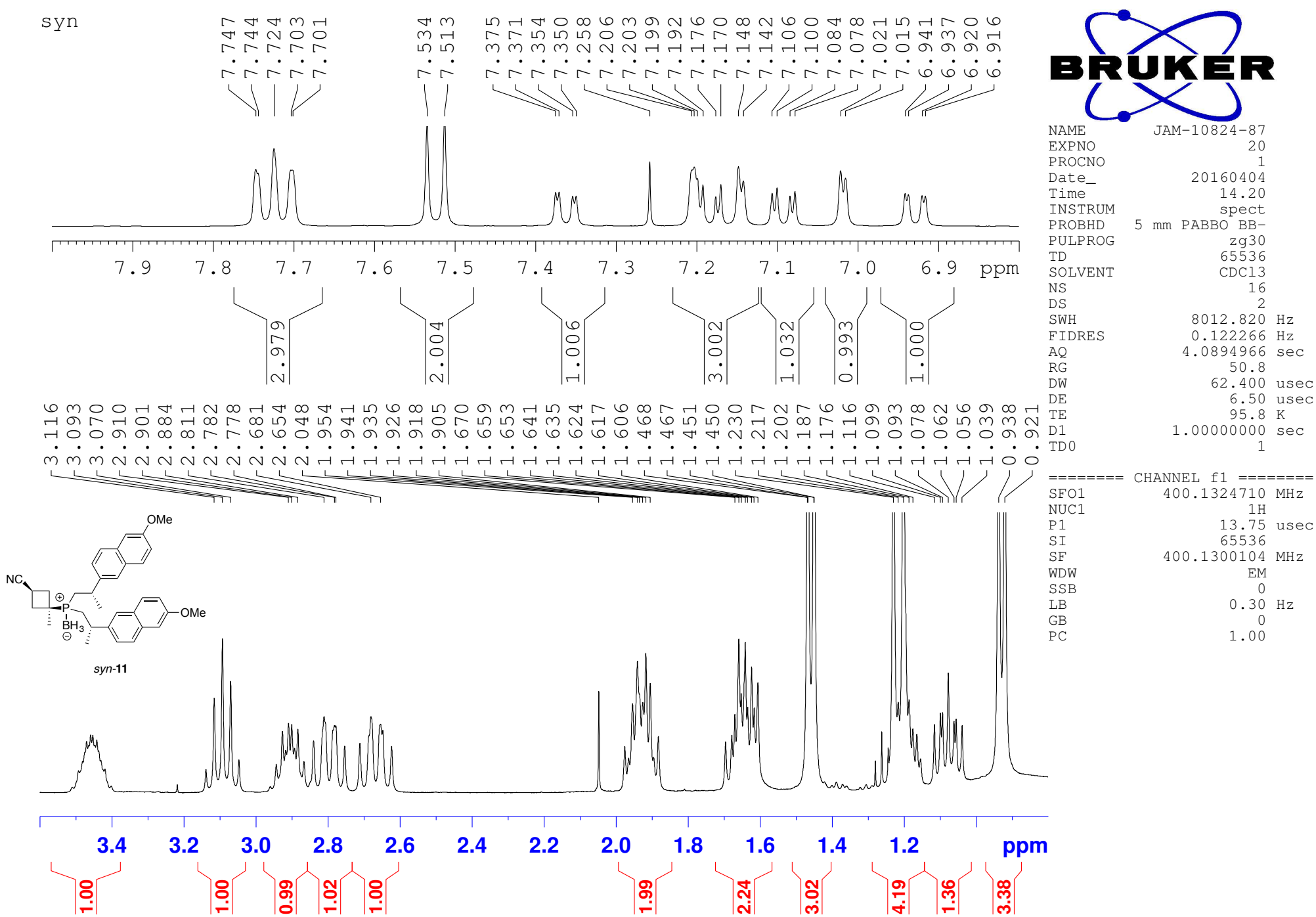
syn

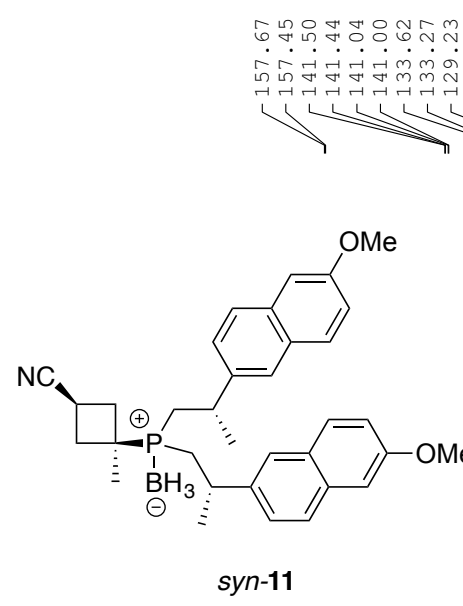

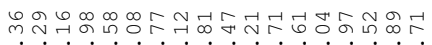

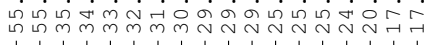
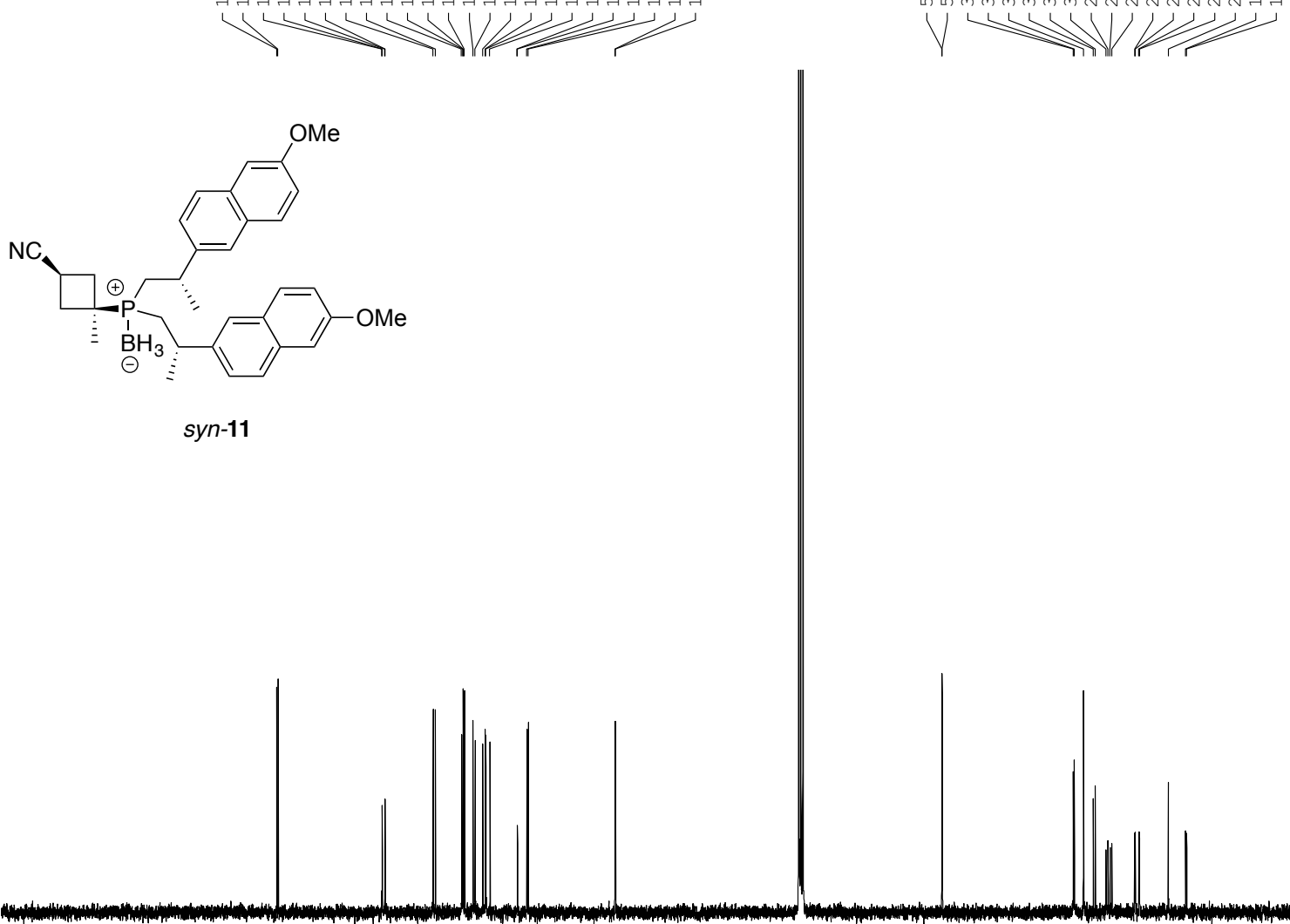

140

120
100

80

60

40

20

ppm 


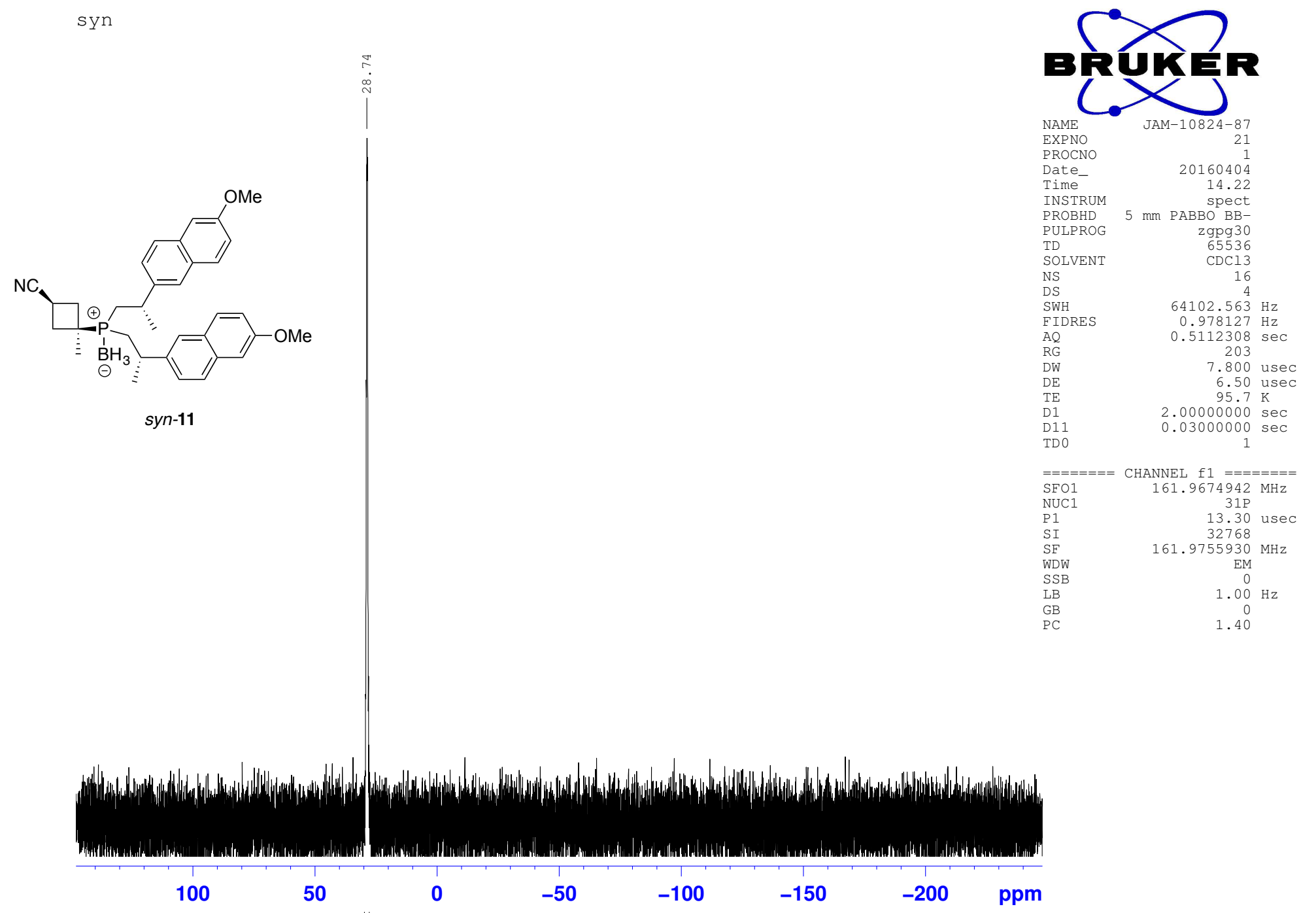




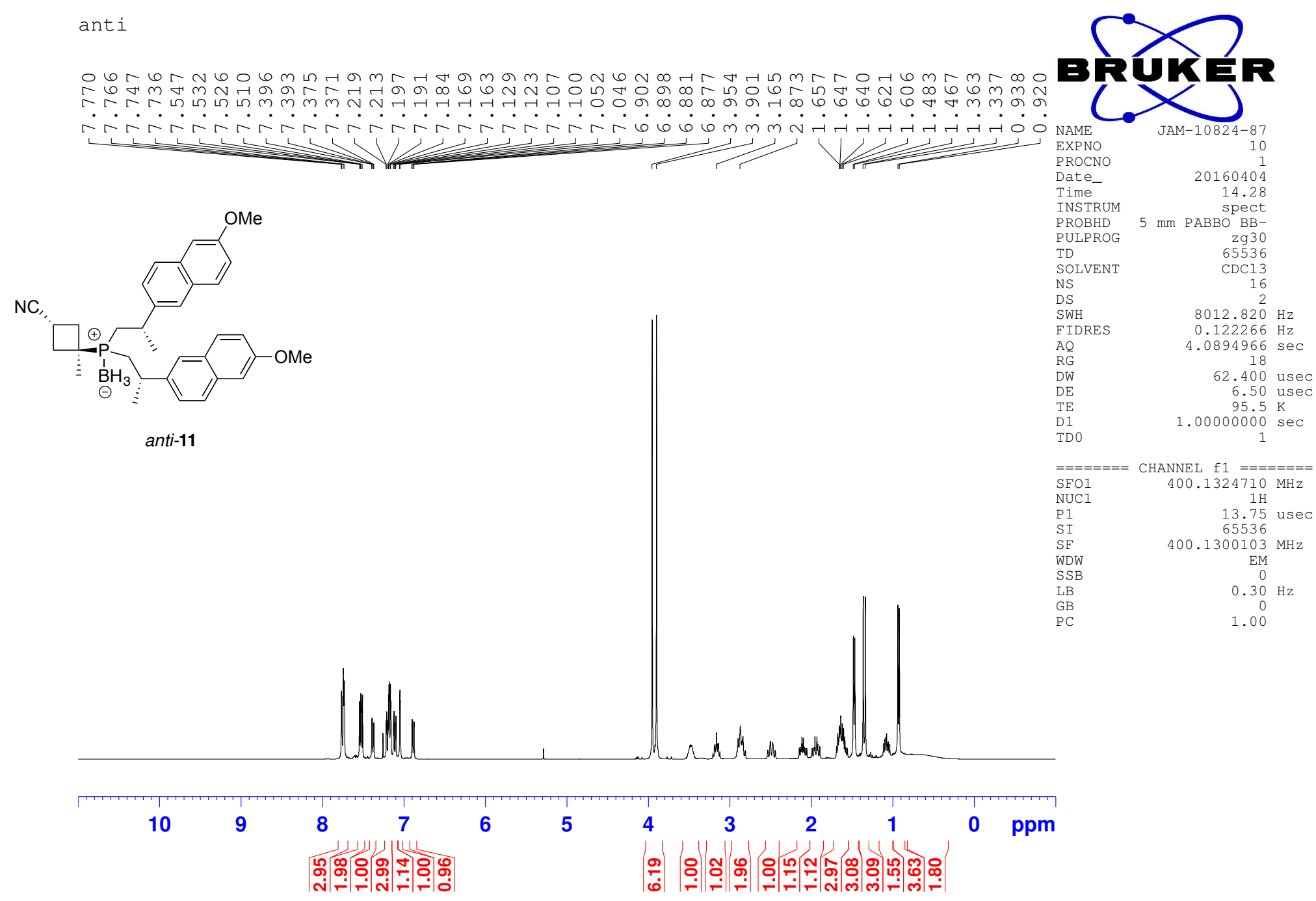




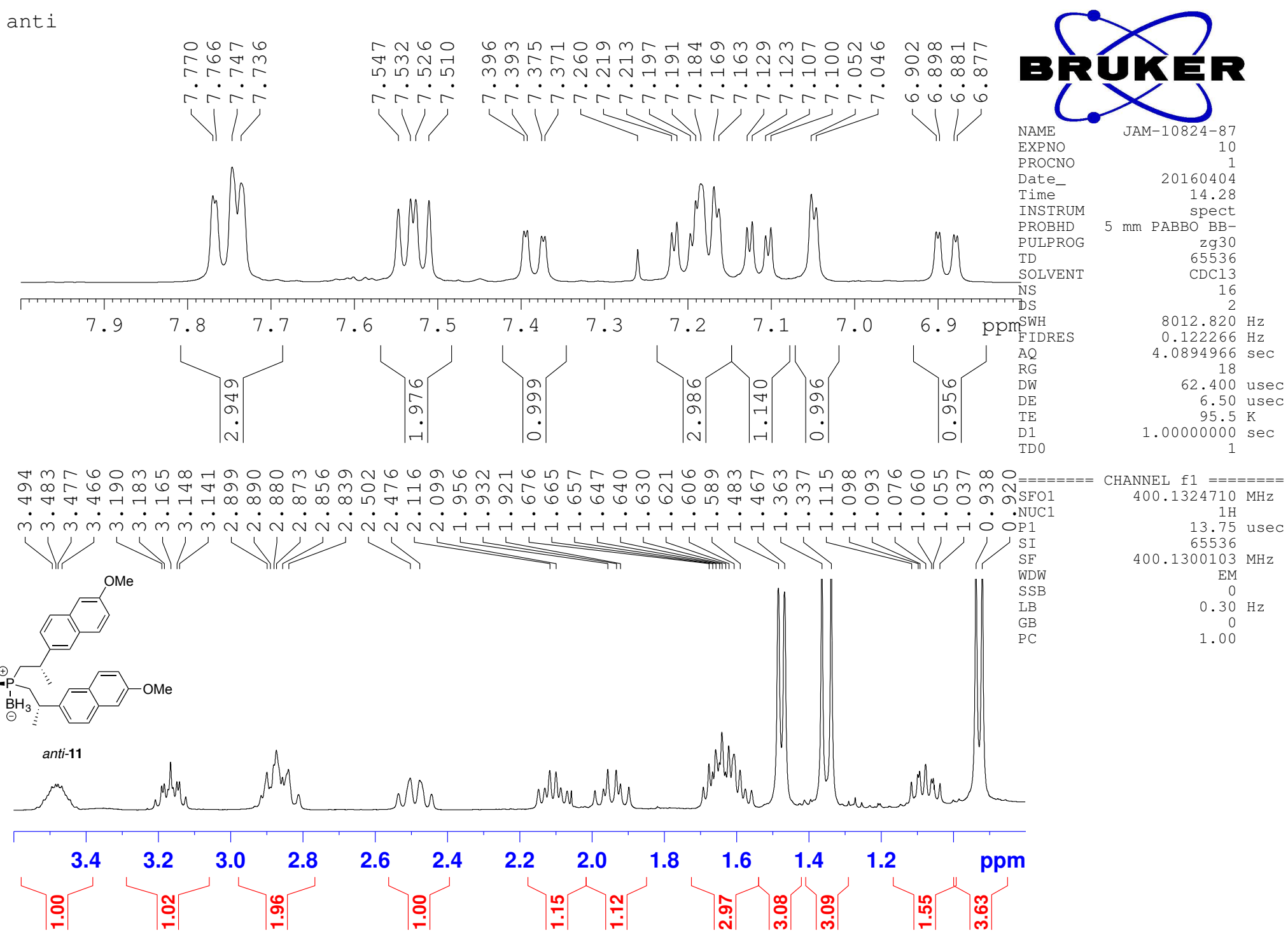


anti

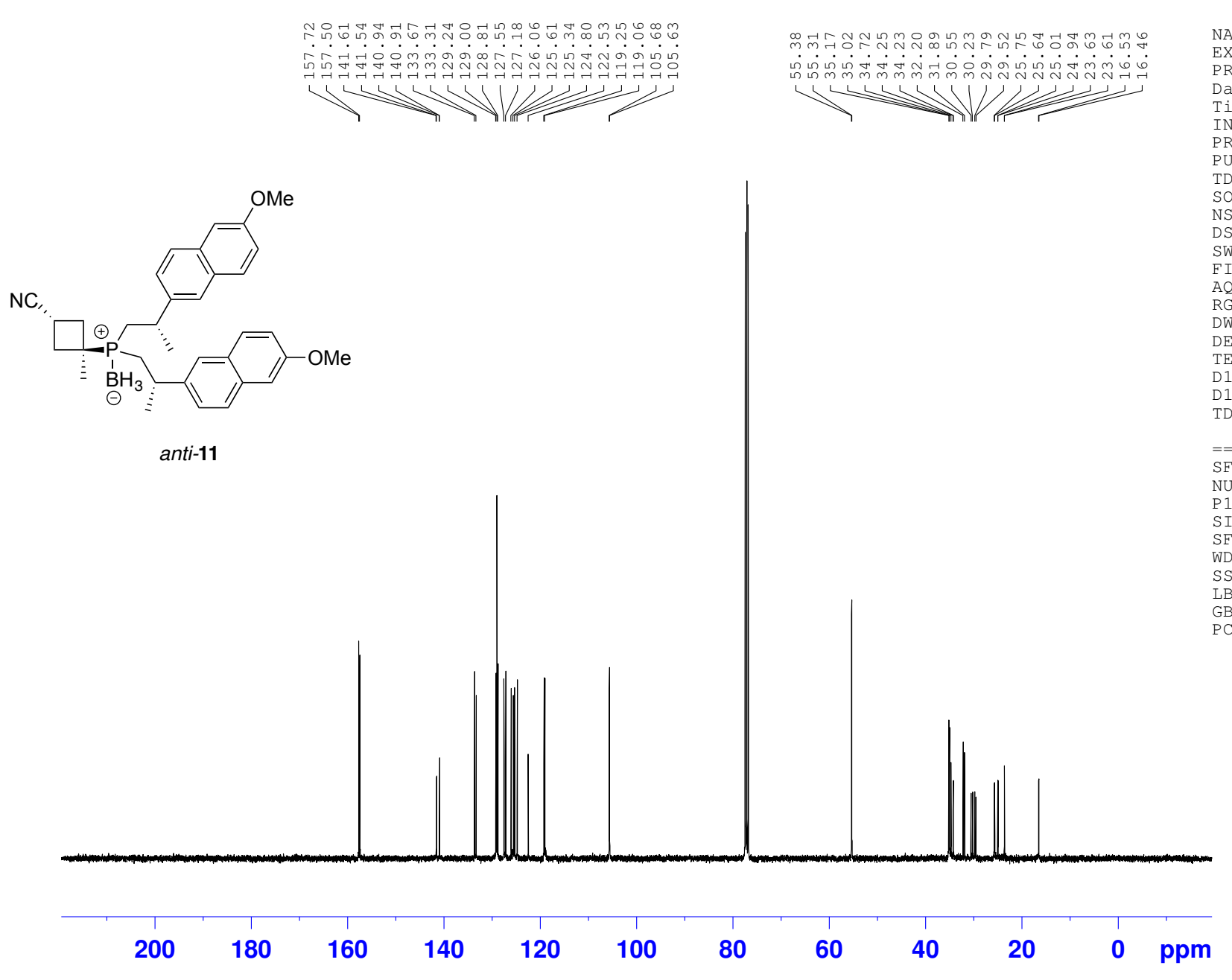

?

NAME

EXPNO

Time-

NSTRUM

$5 \mathrm{~mm}$ PABBO $\mathrm{BB}-$

TD

LVENT

SW

$24038.461 \mathrm{~Hz}$
HDRES
$0.366798 \mathrm{~Hz}$

$1.3631988 \mathrm{sec}$

20.800 usec

6.50 usec

$2.00000000 \mathrm{sec}$ $0.03000000 \mathrm{sec}$

$======$ CHANNEL $\mathrm{f} 1 \mathrm{f}=======$

$100.6228293 \mathrm{MHz}$

$\begin{array}{lr}\mathrm{P} 1 & 13 \mathrm{C} \\ \mathrm{SI} & 10.00 \mathrm{usec}\end{array}$

$\quad 100.6127685 \mathrm{MHz}$

0
$1.00 \mathrm{H}$

$1.00 \mathrm{~Hz}$
0
1.40 


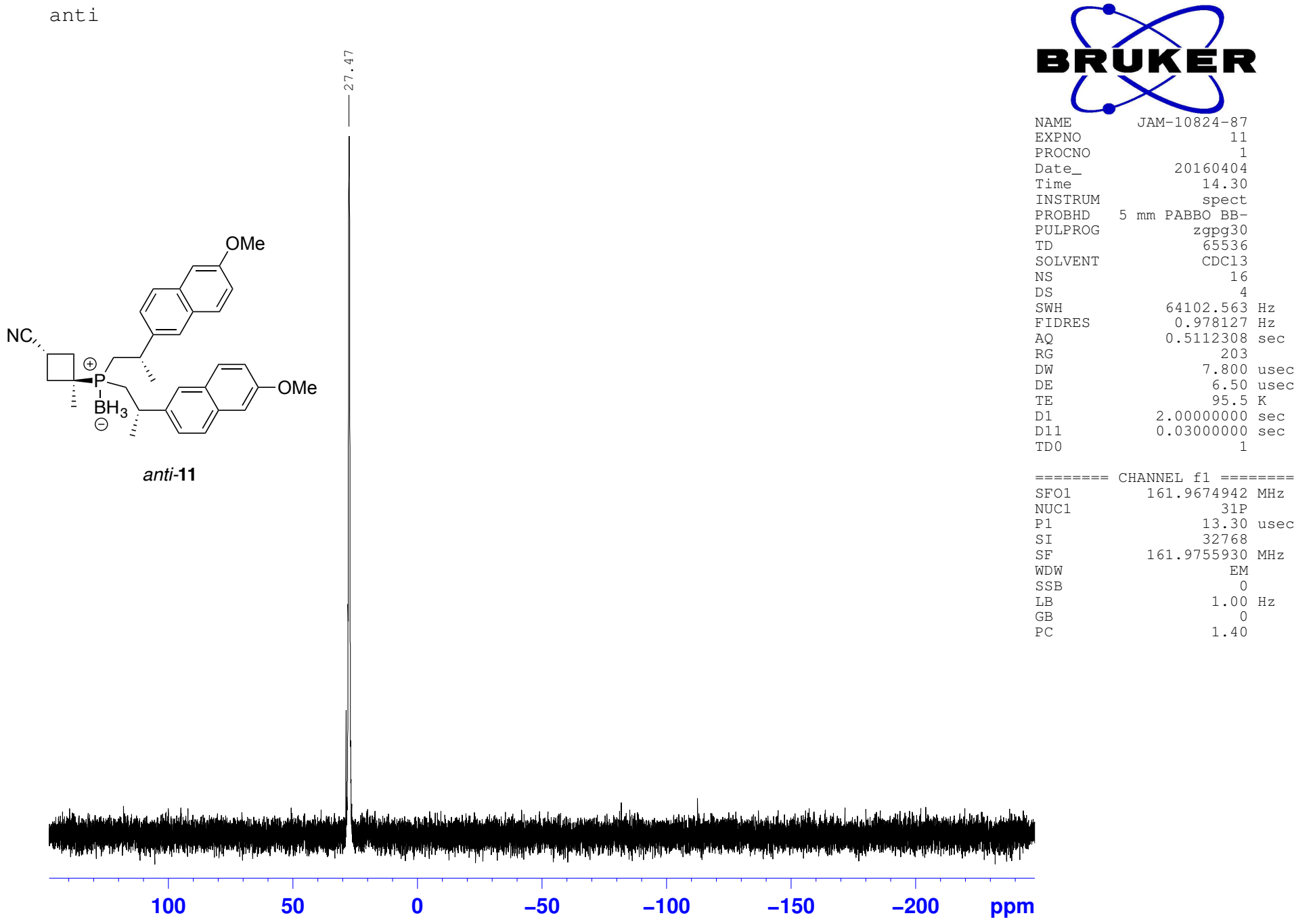




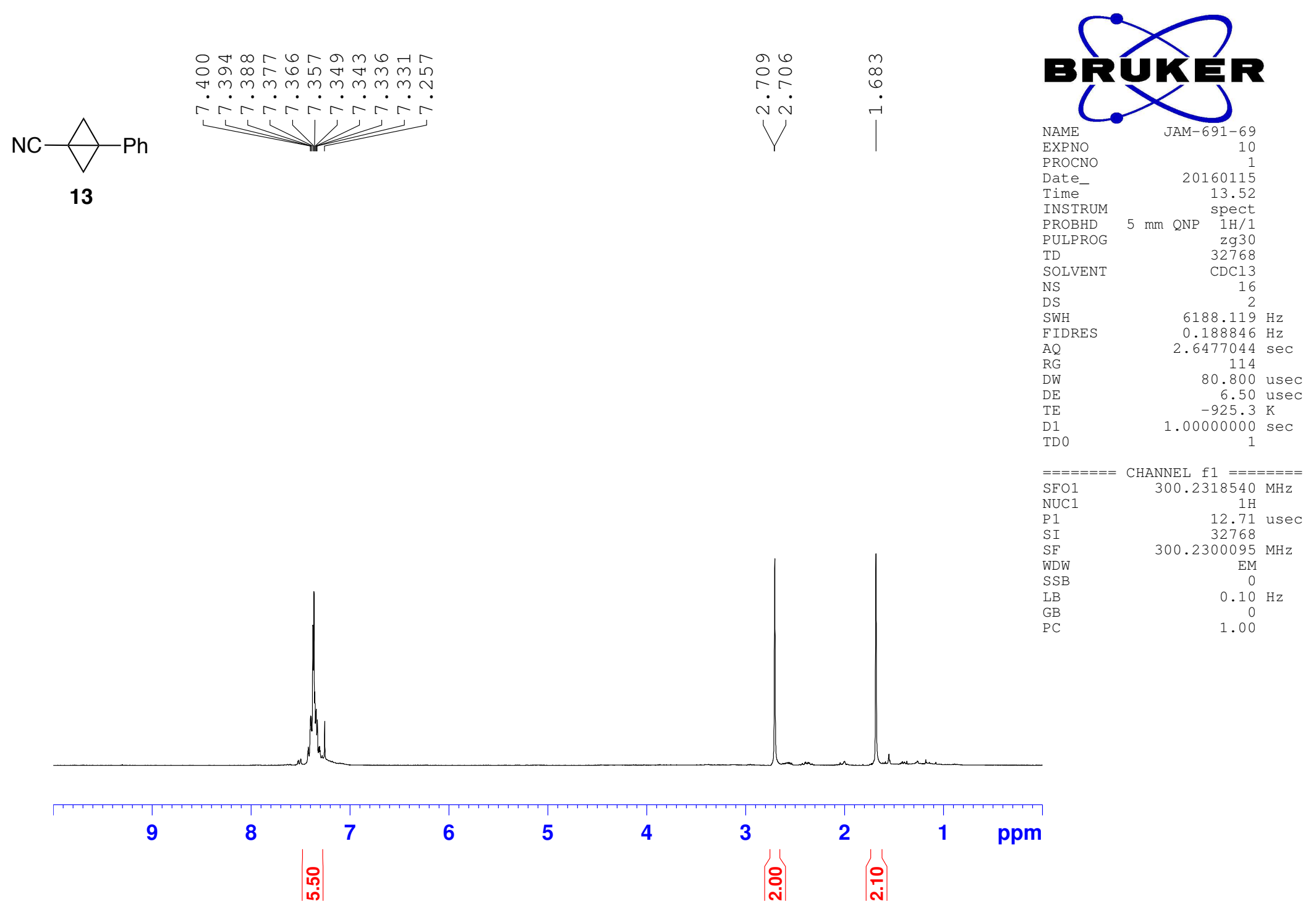


$\mathrm{Ph} \quad \mathrm{BCB} \quad \mathrm{CN}$
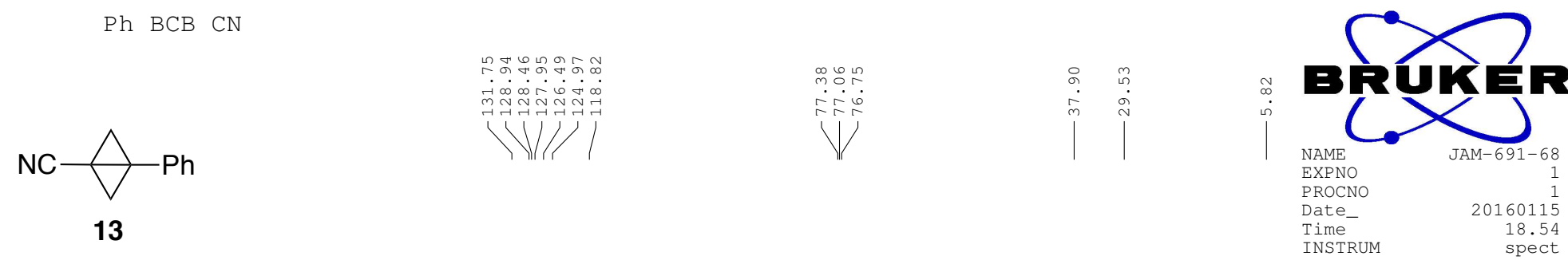

Time-

PROBHD

TD

SOLVENT

NS
DS

FWH

$A Q$

AQ

RG
DW
DE

TE
D1
D11

D11
TD 0

$========$ CHANNEL f1 $=======$

P1

PL1W

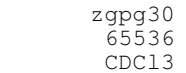

$$
\begin{array}{r}
4 \\
24038.461 \mathrm{~Hz}
\end{array}
$$

$1.3631988 \mathrm{sec}$

20.800 usec
6.50 usec

$.00000000 \mathrm{sec}$

$0.03000000 \mathrm{sec}$

SFO1

10.00 usec

$100.6479773 \mathrm{MHz}$

$=======$ CHANNEL

CPDPRG

PCPD 2
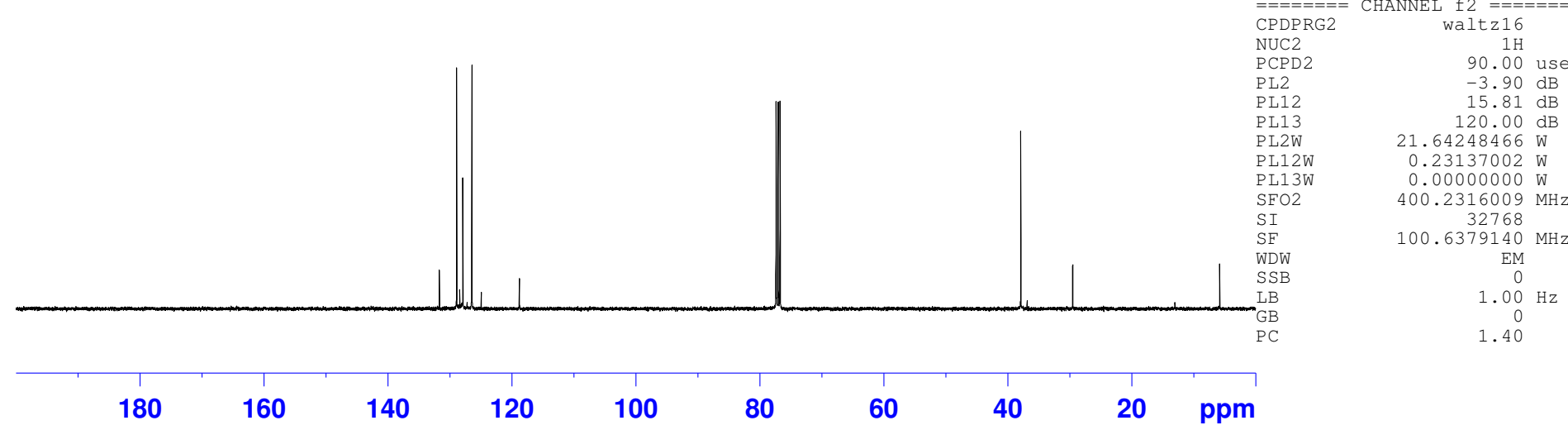


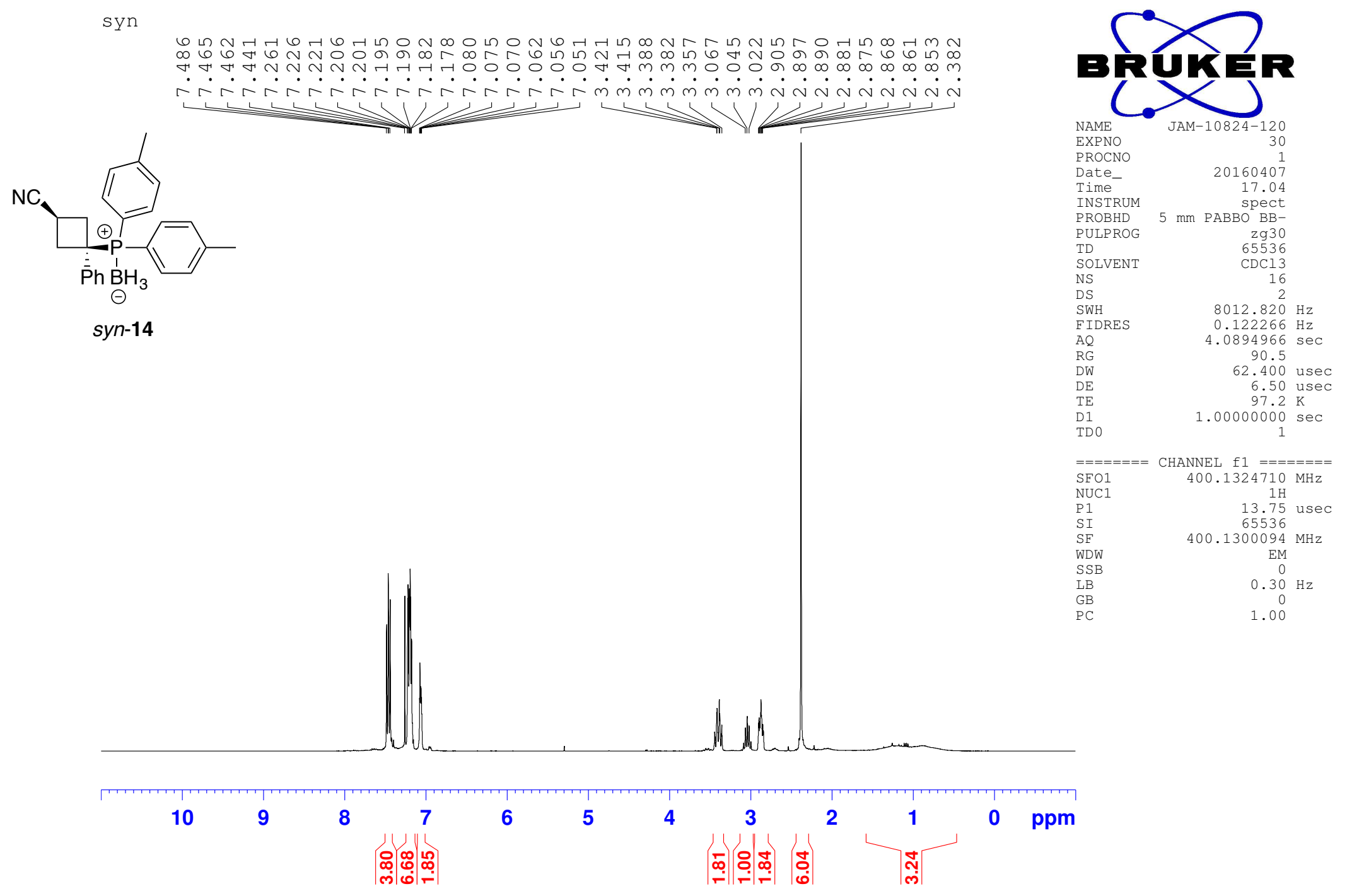


syn $\mathrm{Ph}$ Tol
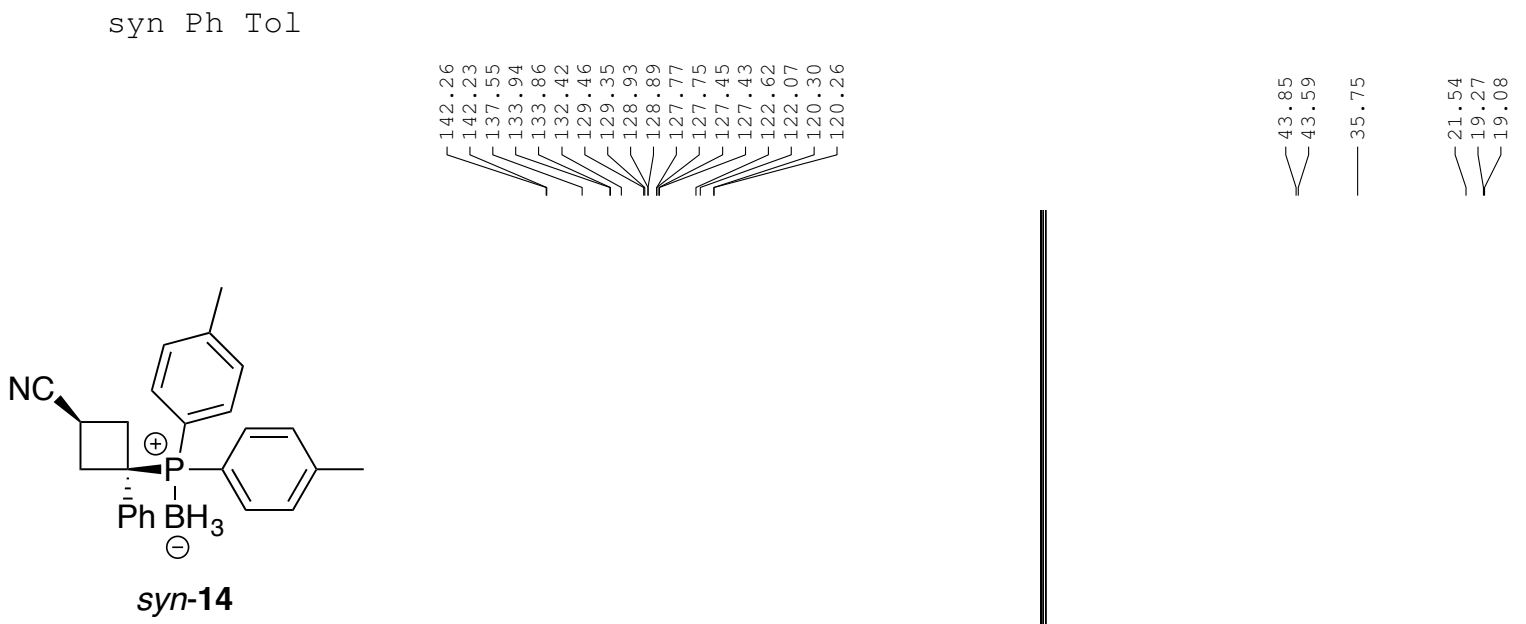

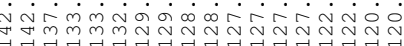
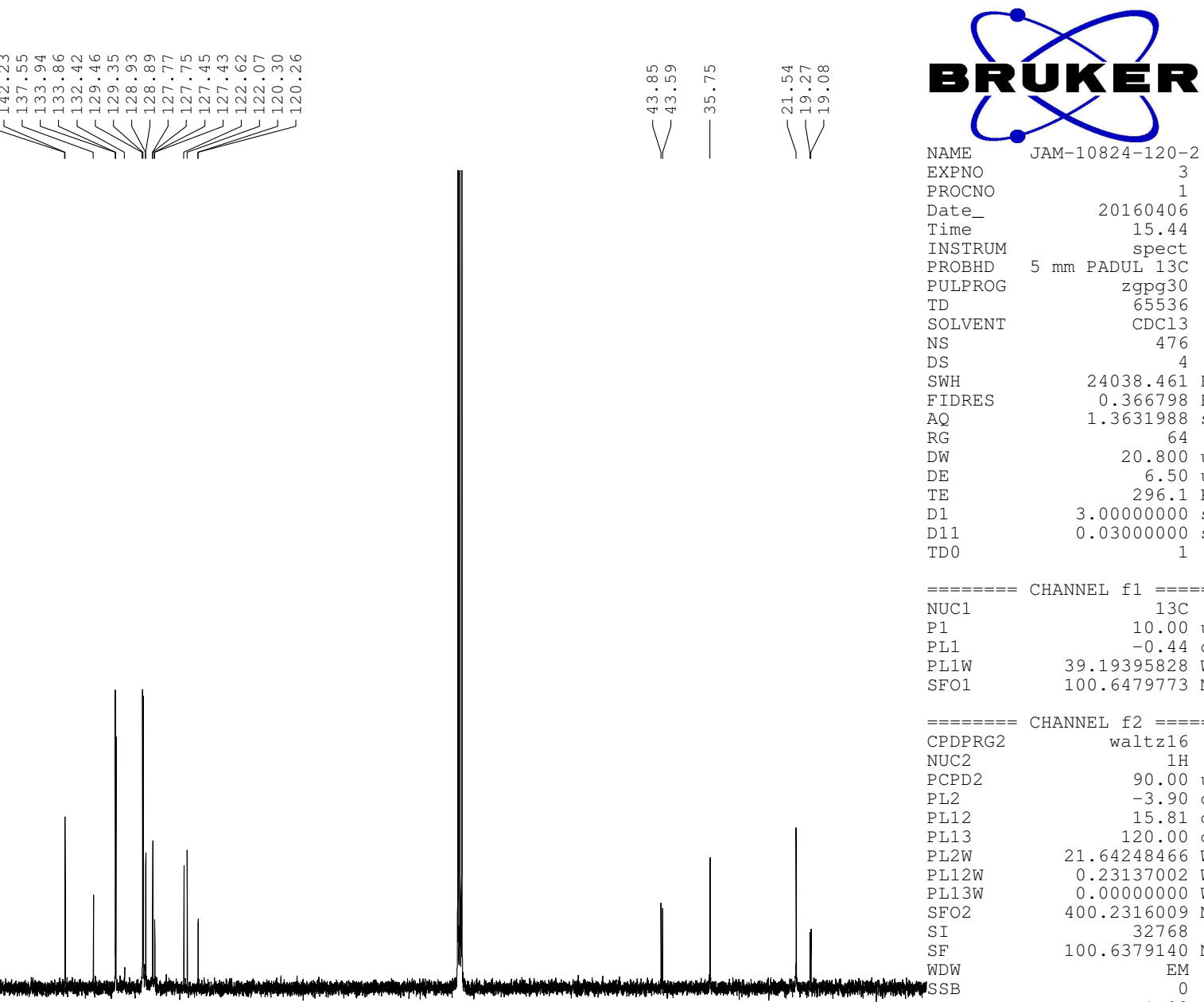
EXPNO

(a)

Date -

$5 \mathrm{~mm}$ PADUL $13 \mathrm{C}$

ULPRO

SOLVENT

NS
DS
SWH

$24038.461 \mathrm{~Hz}$

$2403886798 \mathrm{~Hz}$
$0.3667988 \mathrm{sec}$

RG $\quad 64$ sec

$\begin{array}{lr}\text { DW } & 20.800 \text { usec } \\ \text { DE } & 6.50 \text { usec }\end{array}$

$3.00000000 \mathrm{k}$

$3.00000000 \mathrm{sec}$
D11
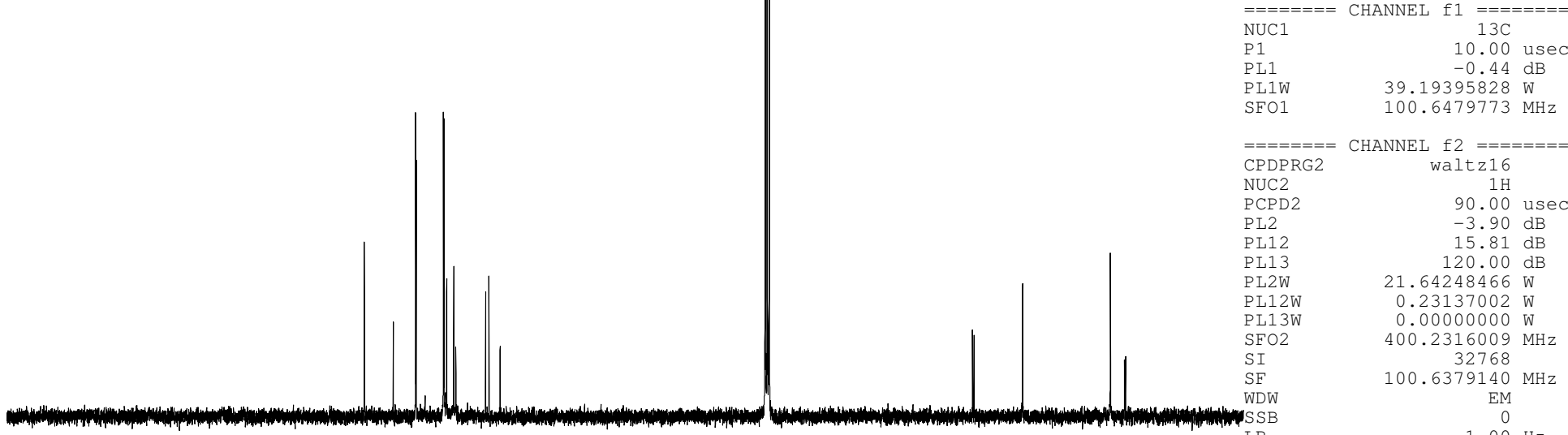

(100.6479773

PDPRG2 CHANNEL 22

CPD2

waltz16

90.00 usec

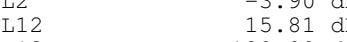

$120.00 \mathrm{~dB}$
$21.64248466 \mathrm{~W}$

$21.64248466 \mathrm{~W}$
$-0.23137002 \mathrm{~W}$

PL13W $0.00000000 \mathrm{~W}$

$\quad 400.2316009 \mathrm{MHz}$

II

LB
GB
PC

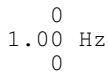

$1.00 \mathrm{~Hz}$
0
1.40 


\section{minor product $\mathrm{Ph} \mathrm{BCB} \mathrm{CN}$ tol $\mathrm{HPN}$ BRuker}

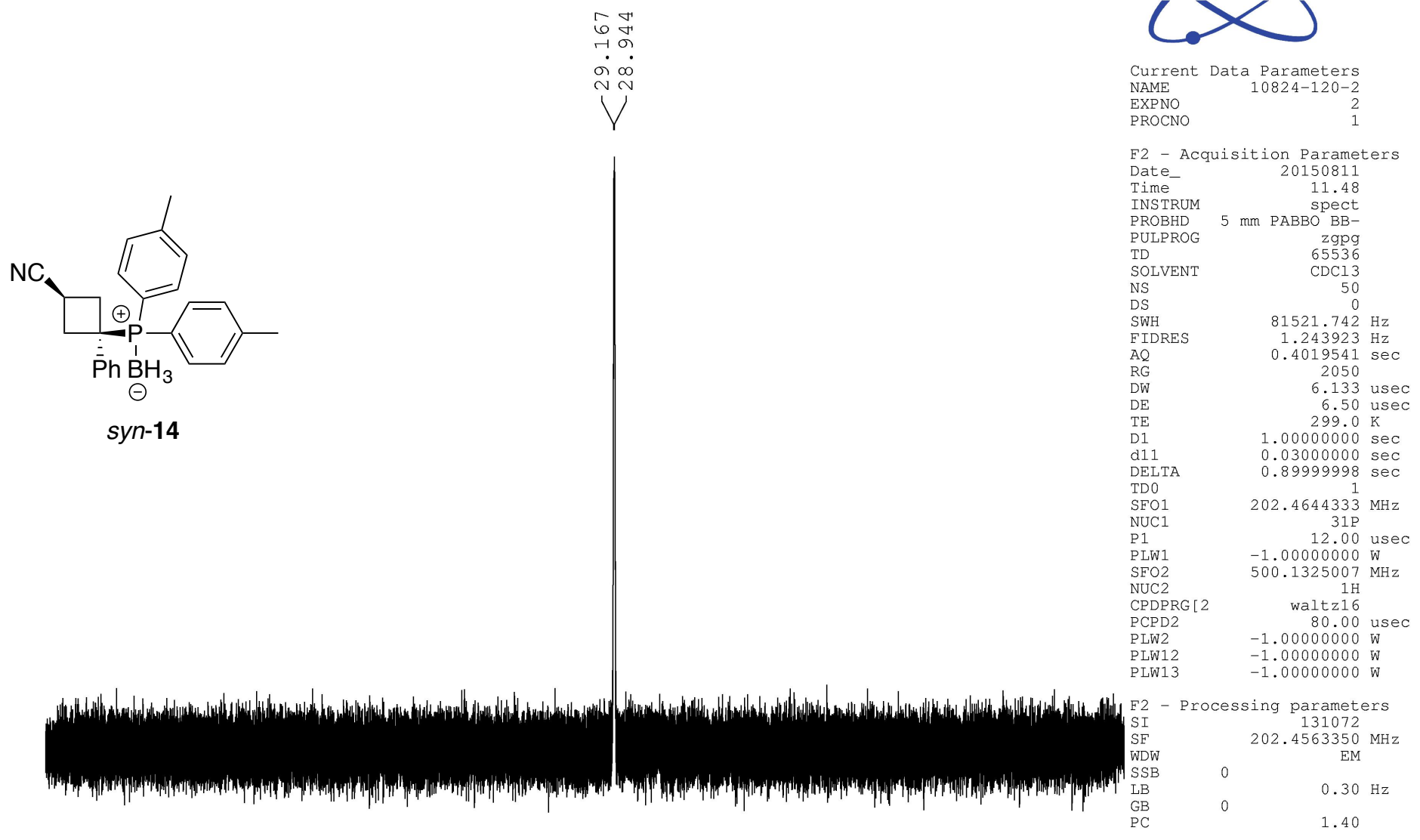




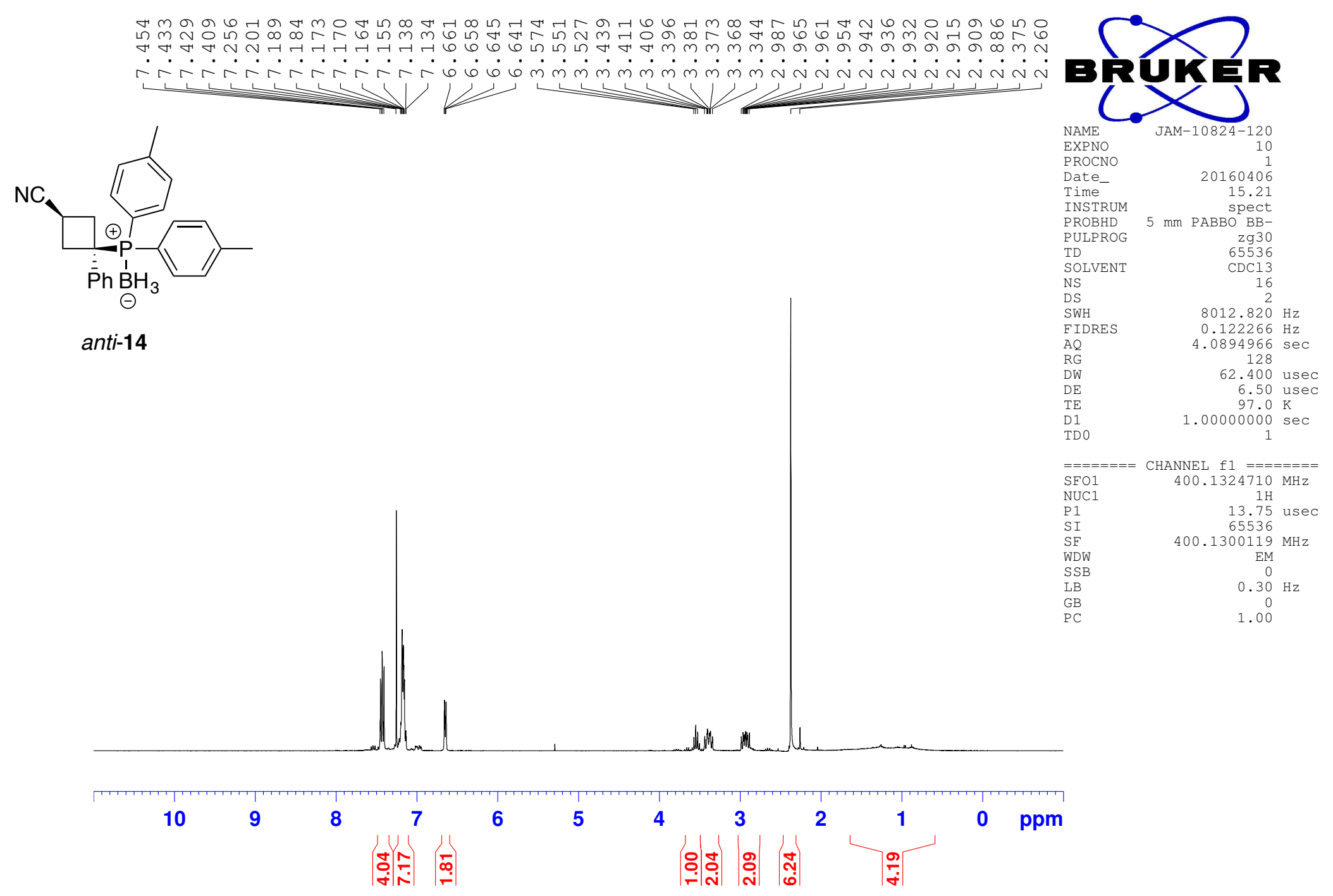



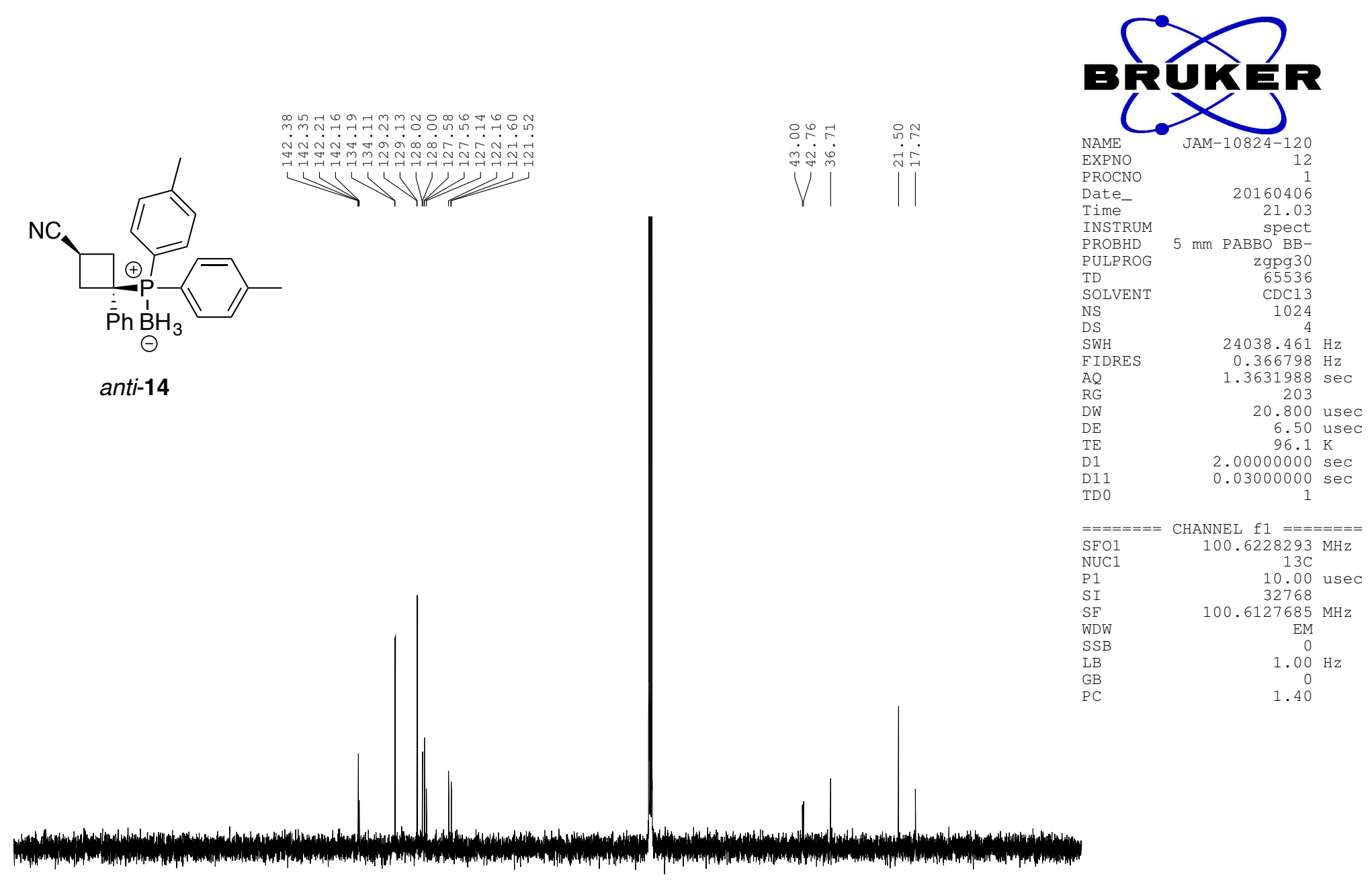

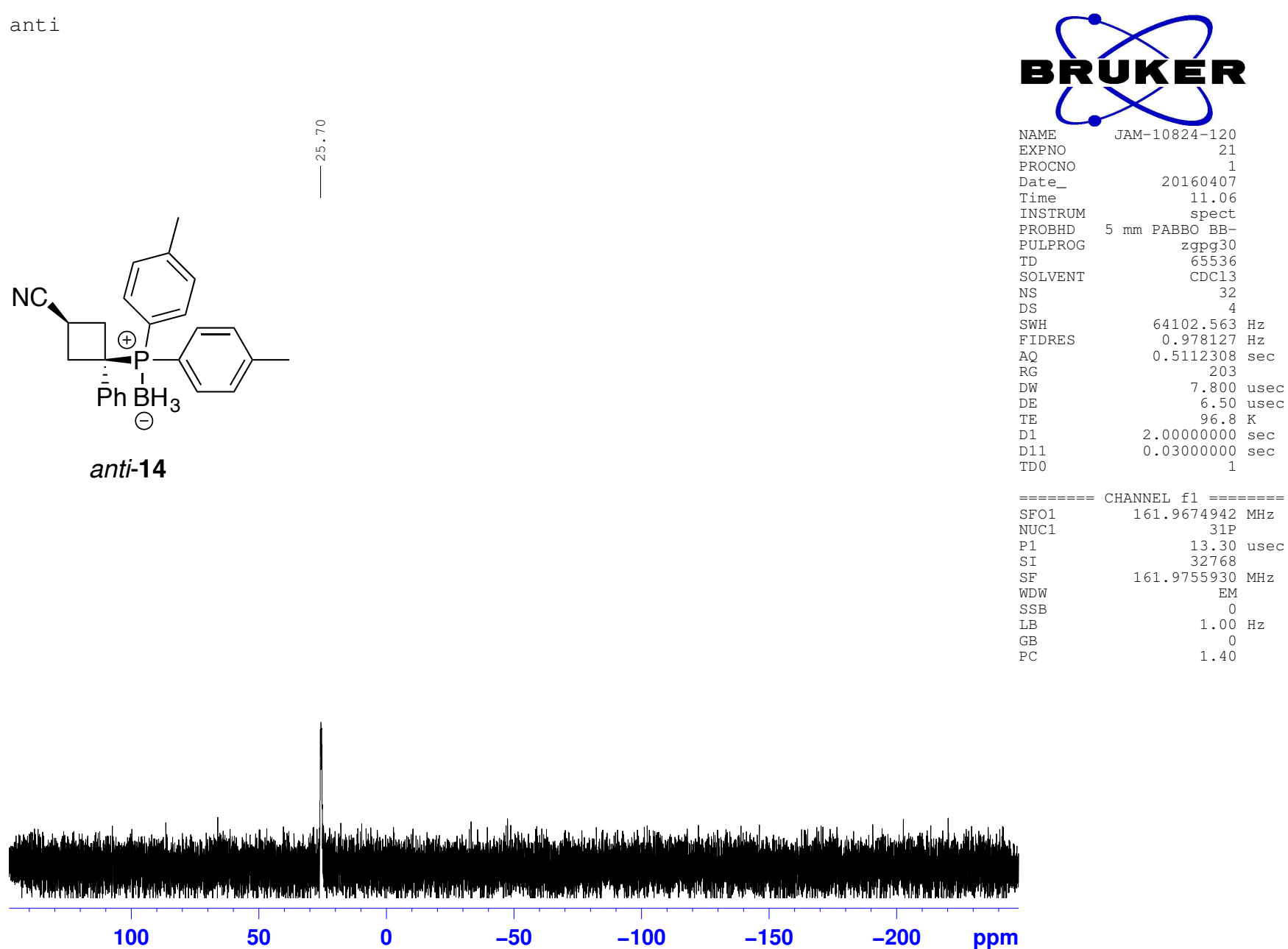


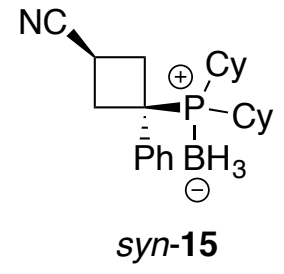

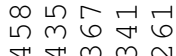

$\therefore r i r$

1/1

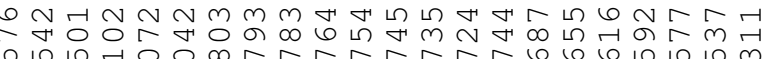

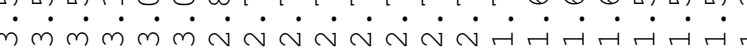
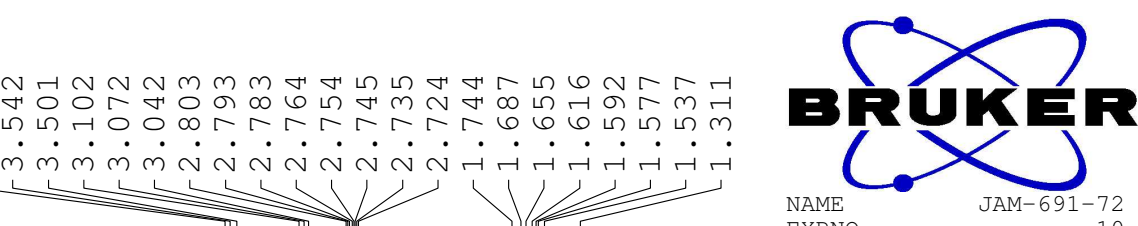

EXPNO

PROCN

Date
Time

$\begin{array}{ll}\text { INSTRUM } & \text { spect } \\ \text { PROBHD } 5 \mathrm{~mm} \text { QNP } & \mathrm{H} / 1\end{array}$

PULPROG

TD

SOLVE
NS

$\begin{array}{lr}\text { DS } & 2 \\ \text { SWH } & 6188.119 \mathrm{~Hz} \\ \text { FIDRES } & 0.188846 \mathrm{~Hz}\end{array}$

$0.188846 \mathrm{~Hz}$
$2.6477044 \mathrm{sec}$

322

80.800 usec

$\begin{array}{lr}\text { DE } & 6.50 \mathrm{usec} \\ \text { TE } & -925.6 \mathrm{~K} \\ \text { D1 } & 1.00000000 \mathrm{sec}\end{array}$

TD 0
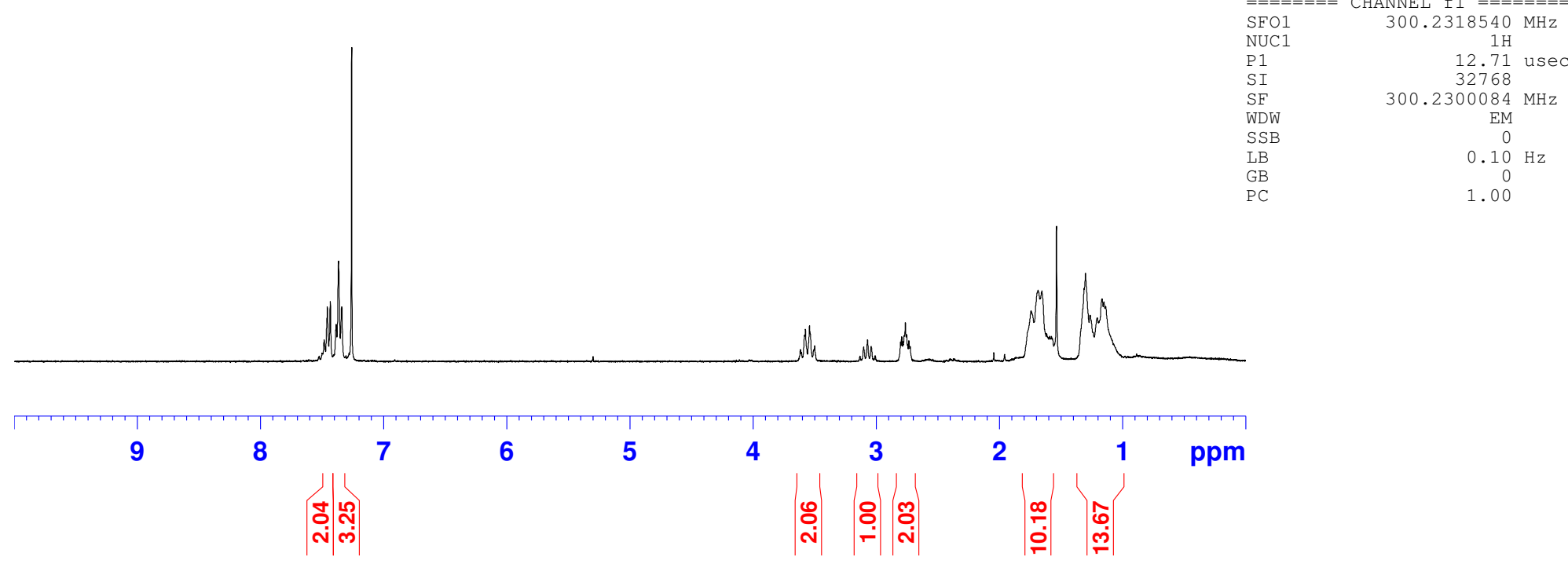


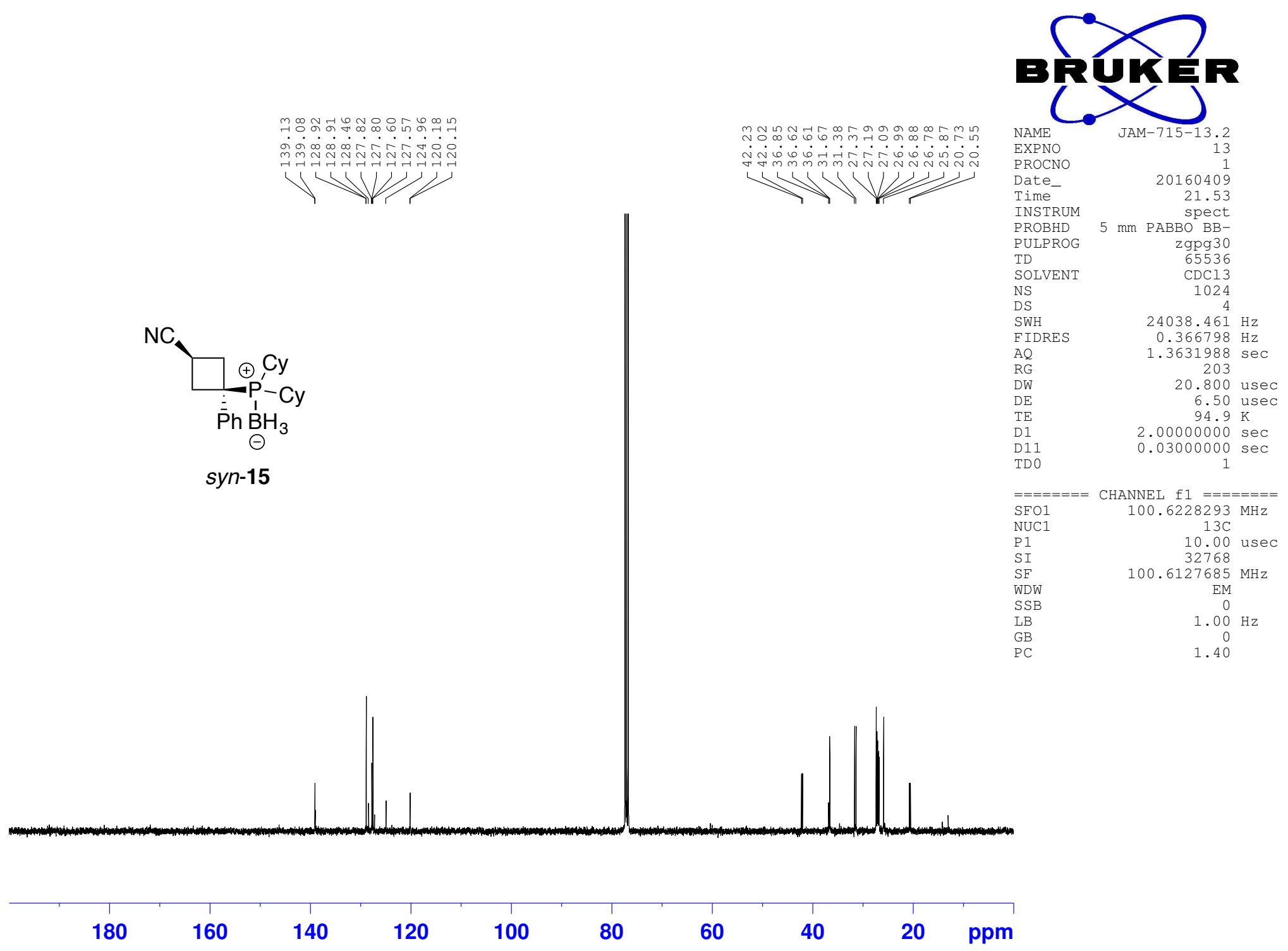



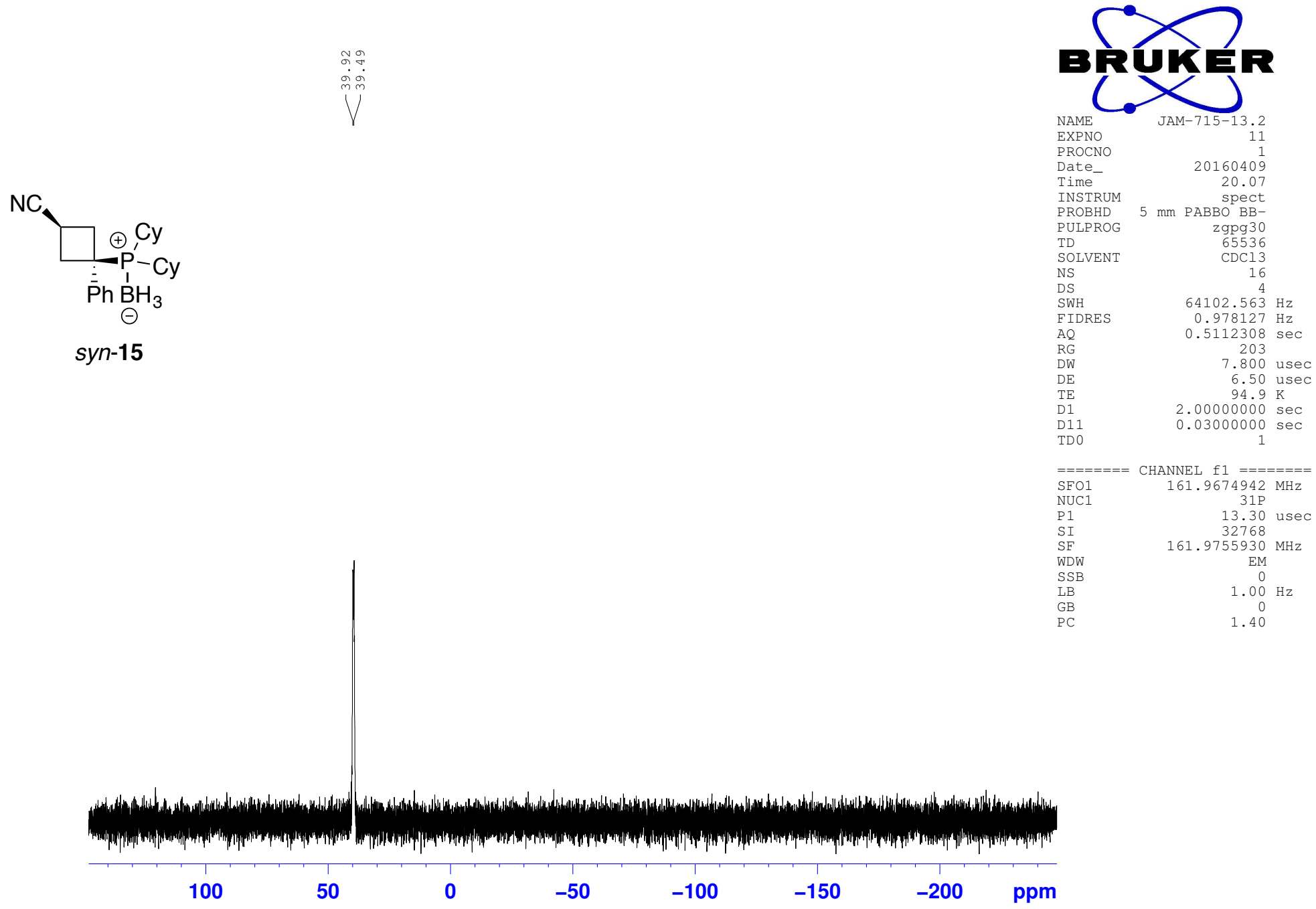


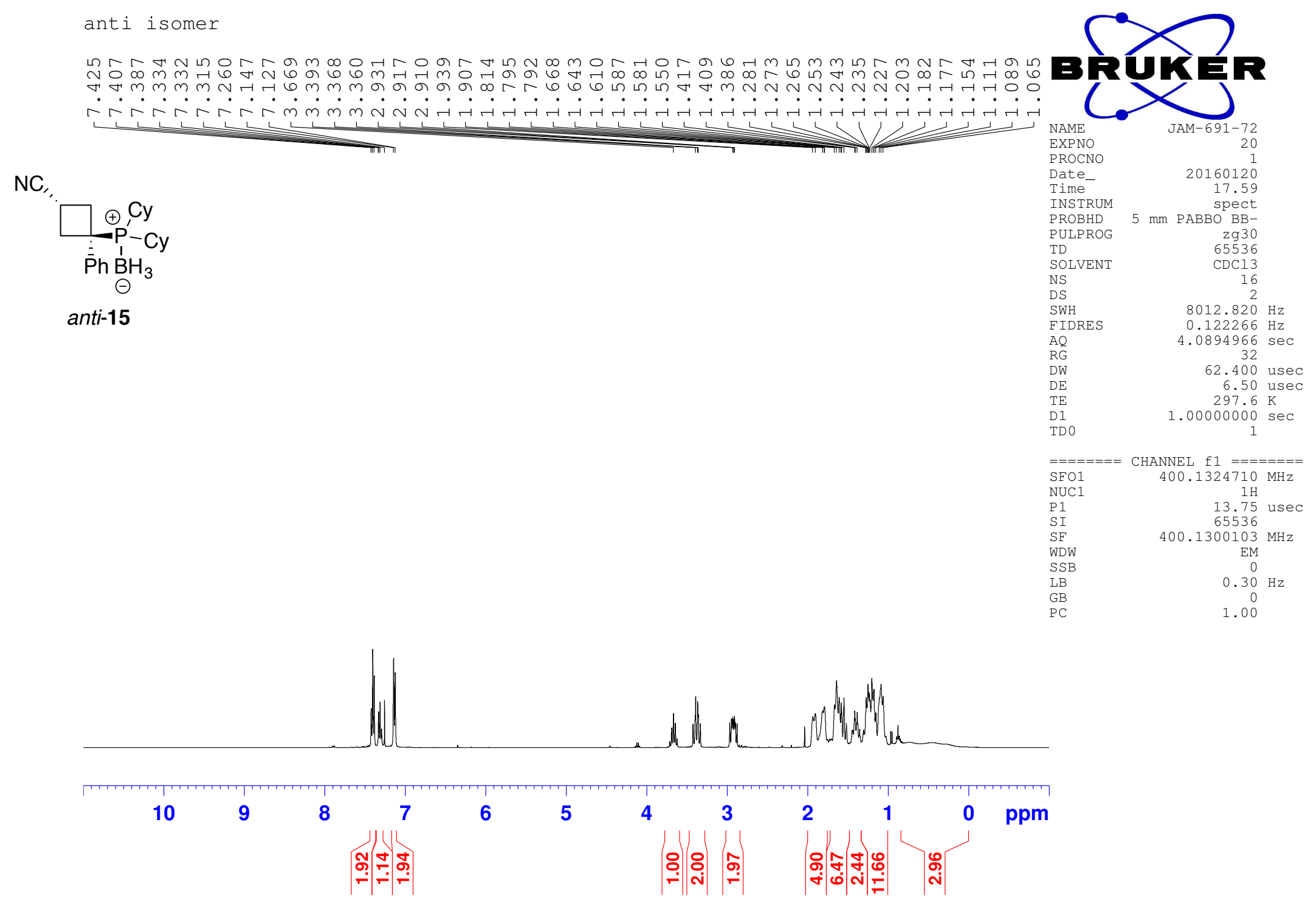


anti isomer

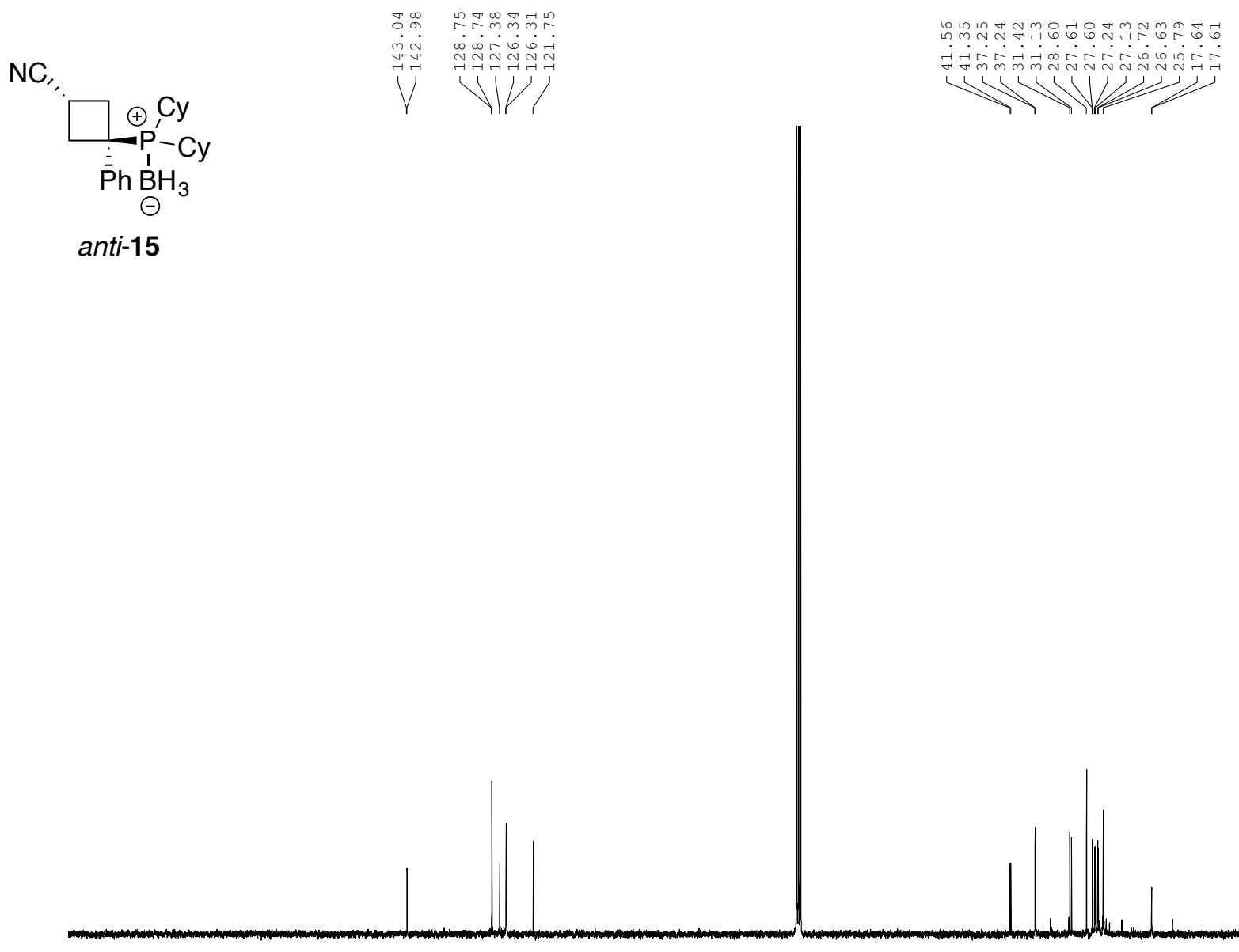

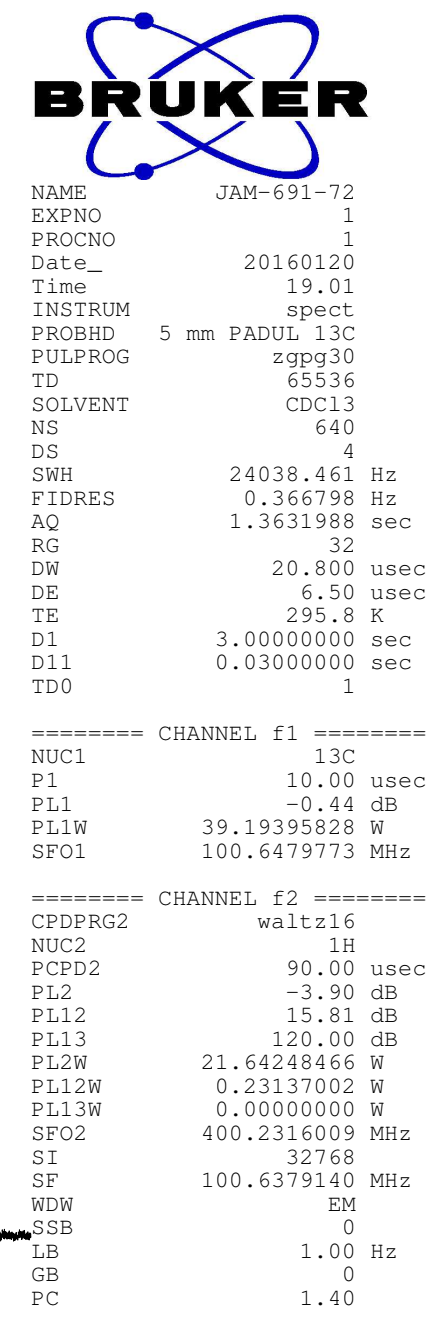


anti isomer

NC,

$$
\begin{aligned}
& \square \stackrel{\square_{1}^{\prime}}{\mathrm{Cy}} \\
& \overline{\mathrm{Ph} \mathrm{BH}} \mathrm{B}_{3} \\
& \begin{array}{r}
\Theta \\
\text { anti-15 }
\end{array} \\
& \text { anti-15 }
\end{aligned}
$$

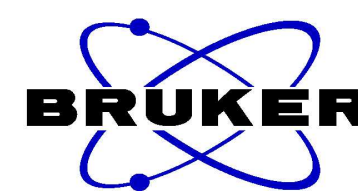

NAME EXPNO

$\begin{array}{lr}\text { PROCNO } & 20160120 \\ \text { Date } & 17.57 \\ \text { Time } & 17.57 \\ \text { INSTRUM } & \text { spect }\end{array}$

PROBHD $5 \mathrm{~mm}$ PABBO BB-

PULPROG $\quad$ zgpg30

TD

SOLVE

DS

$\begin{array}{lr}\text { SWH } & 64102.563 \mathrm{~Hz} \\ \text { FIDRES } & 0.978127 \mathrm{~Hz}\end{array}$

AQ $\quad 0.5112308 \mathrm{sec}$

$\begin{array}{lr}\text { RG } & 203 \\ \text { DW } & 7.800 \text { usec }\end{array}$

$\begin{array}{lr}\text { DE } & 6.50 \text { use } \\ \text { TE } & 297.7 \mathrm{~K}\end{array}$

$=======$ CHANNEL $\mathrm{f} 1 \mathrm{l}=======$

SFO1 $161.9674942 \mathrm{MHz}$

NUC1 $11 \mathrm{P}$

$\begin{array}{lr}\text { P1 } & 13.30 \text { use } \\ \text { SI } & 32768 \text { us }\end{array}$

SF $\quad 161.9755930 \mathrm{MHz}$

SSB

$\begin{array}{lr}\text { LB } & 1.00 \\ \text { GB } & 0 \\ \text { PC } & 1.40\end{array}$

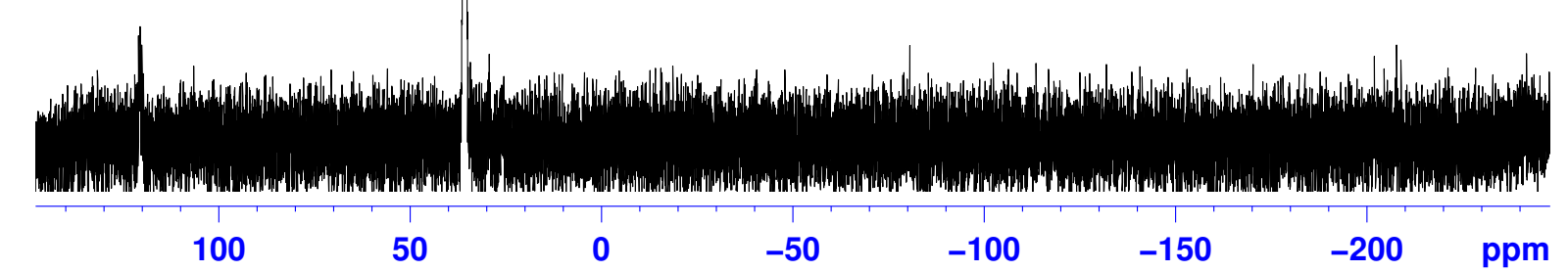




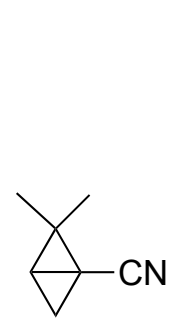

$(+/-)-16$
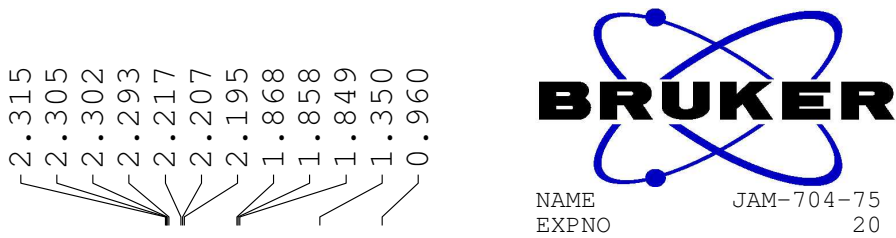

EXPNO

Date

INSTRUM

PROBHD

PULPROG

SOLVENT

NS
DS

SWH $\quad 6188.119 \mathrm{~Hz}$

2. $6477846 \mathrm{~Hz}$

$\begin{array}{ll}\text { AQ } & 2.6477846 \\ \text { RG } & 203 \\ \text { DW } & \end{array}$

80.800 usec

6.50 use

$\begin{array}{lr}\text { TE } & -925.5 \mathrm{~K} \\ \text { D1 } & 1.00000000 \mathrm{sec}\end{array}$

TDO

$=======$ CHANNEL $\mathrm{f} 1 \mathrm{l}======$
SFO1 $300.2318540 \mathrm{MHz}$

SFO1
NUC1

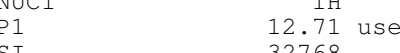

WDW $\quad 300.2300091 \mathrm{MHz}$

SSB

LB

0

$0.10 \mathrm{~Hz}$

0
1.00

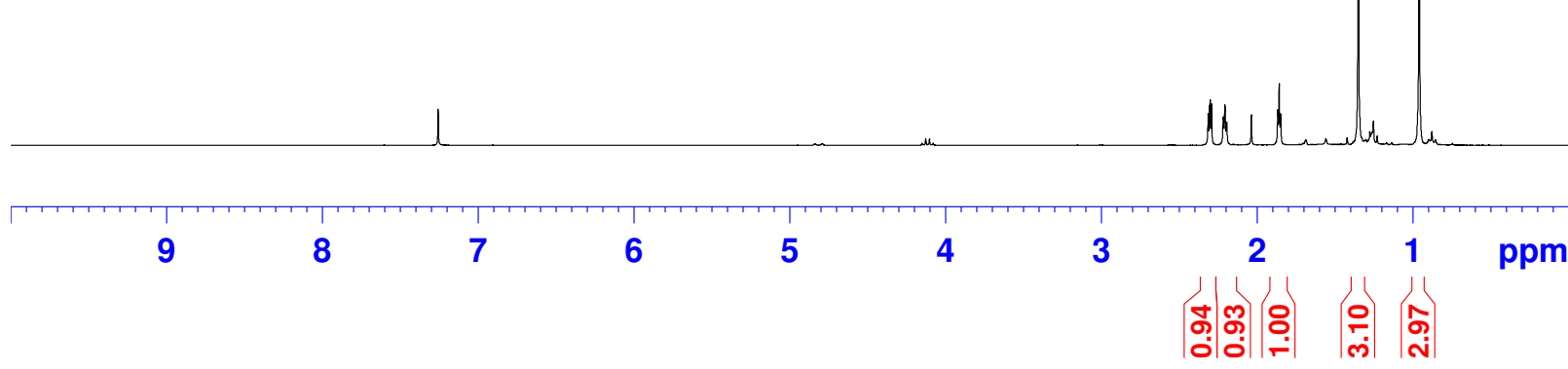




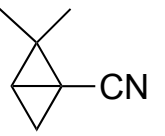

$(+/-)-16$
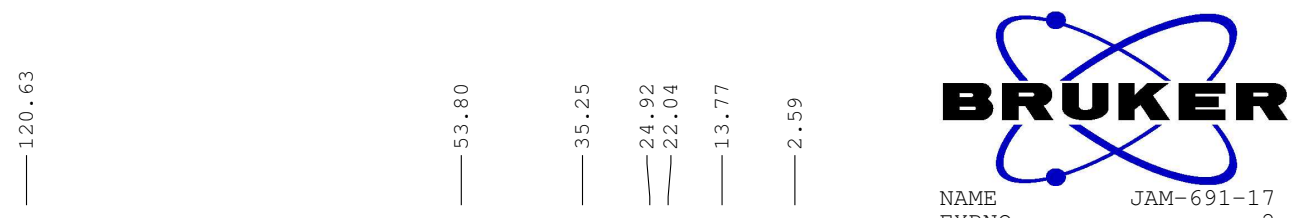

NAME
EXPNO

PROCNO

Time

INSTRUM

PROBHD
PULPROG

TD

SOLVENT

NS
DS

SWH
FIDRES

FIDR
AQ
RG
DW

$\mathrm{RG}$
$\mathrm{DW}$
$\mathrm{DE}$
$\mathrm{TE}$

D1
D11

D11
TD 0

20151202
10.42

spect
$\mathrm{mm}$ PADUL $13 \mathrm{C}$
zgpg 30

435
4

$24038.461 \mathrm{~Hz}$

$1.3631988 \mathrm{sec}$

45.2
20.800 use
6.50

6.50 usec

$295.6 \mathrm{~K}$

$3.00000000 \mathrm{sec}$
$0.03000000 \mathrm{sec}$

$=======$ CHANNEL $\mathrm{f} 1$

P1

$\begin{array}{lr}\text { PL1 } & -0.44 \mathrm{~dB} \\ \text { PL1W } & 39.19395828 \mathrm{~W} \\ \text { SFO1 } & 100.6479773 \mathrm{MHz}\end{array}$

1 sec

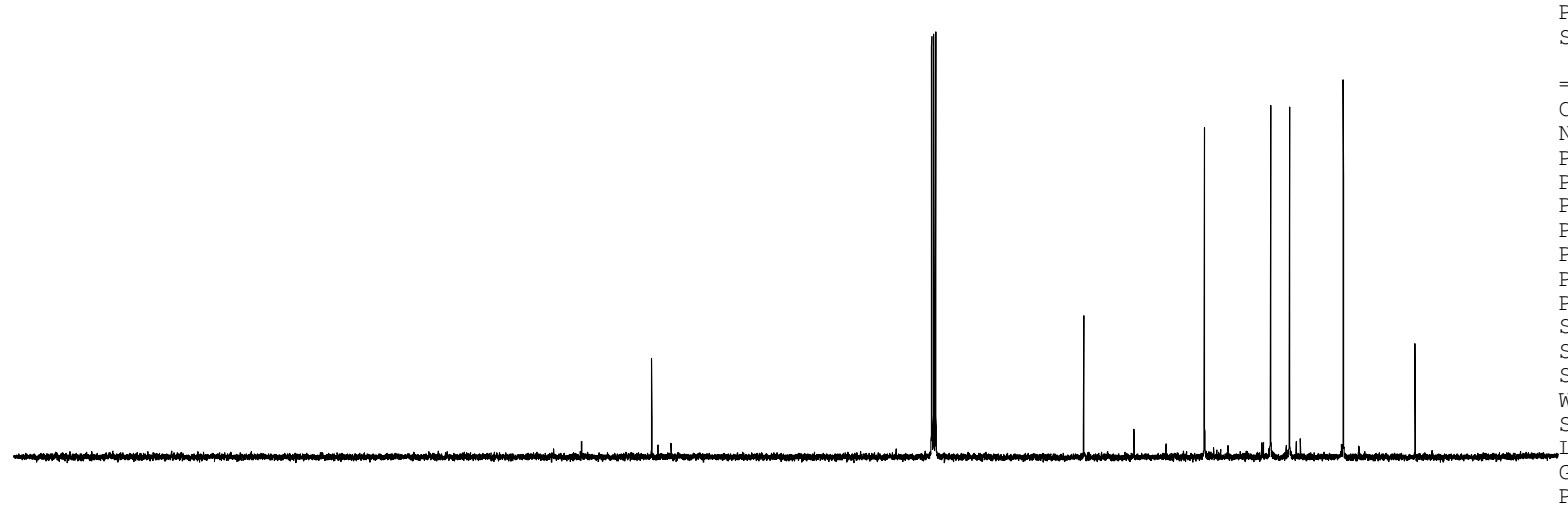

CHANNEL $\mathrm{f} 2======$

NUC2

PCPD 2

PL2
PL12

PL13

PL12W

$\mathrm{SF}$

WDW

GB
PC

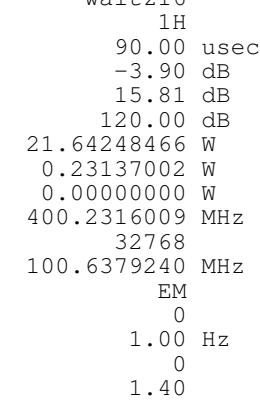




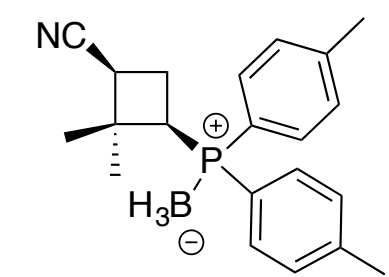

syn-17

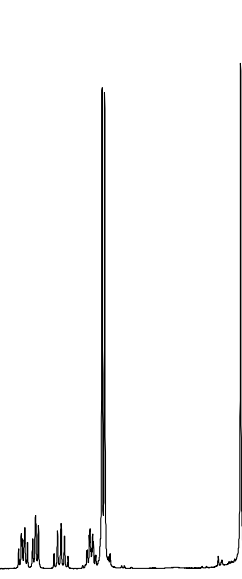

109

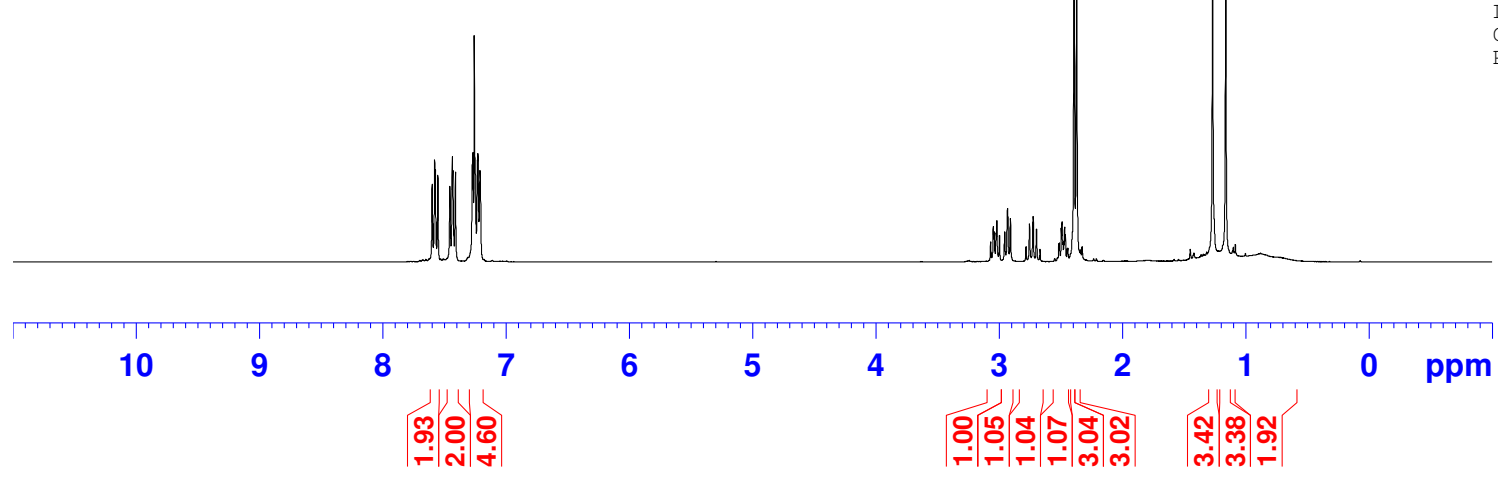
คั:
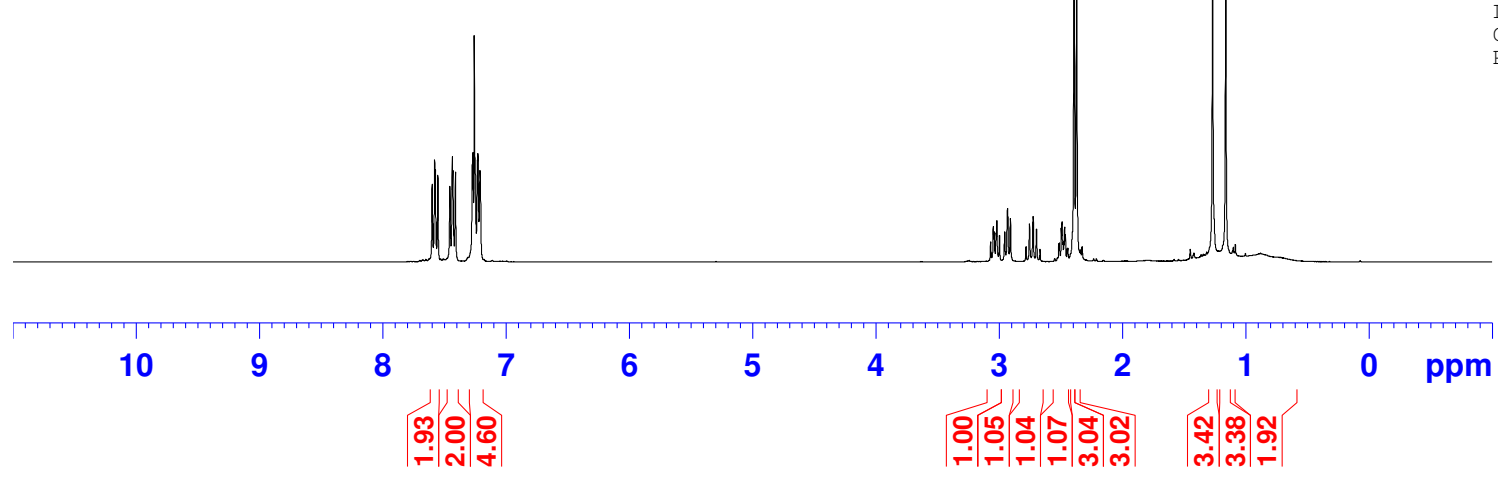

0 ppm

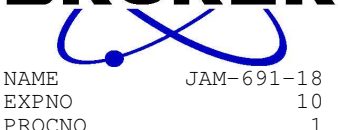

EXPNO
PROCNO
Date-

$\begin{array}{lr}\text { Date } & 20160408 \\ \text { Time } & 13.42 \\ \text { TNSTRUM } & \text { spect }\end{array}$

spect
INSTRUM
PROBHD $5 \mathrm{~mm}$ PABBO BB-

PULPROG

TD
SOLVENT

NS
DS
SWH

DS
SWH
FIDRES

AQ

RG

DW
DE
TE
D1
TDO

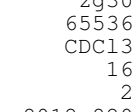

16
2
8012.820
0.122266

$0.122266 \mathrm{~Hz}$
$4.0894966 \mathrm{sec}$

90.5

6.50 usec
$95.9 \mathrm{~K}$

$\begin{array}{ll}\text { D1 } & 1.00000000 \mathrm{~K} \\ \text { TDO } & \end{array}$

$\begin{array}{lc}======= & \text { CHANNEL } \mathrm{f1}======= \\ \text { SFO1 } & 400.1324710 \mathrm{MHz} \\ \text { NUC1 } & 1 \mathrm{H} \\ \text { P1 } & 13.75 \mathrm{usec} \\ \text { SI } & 65536 \\ \text { SF } & 400.1300105 \mathrm{MHz} \\ \text { WDW } & \text { EM } \\ \text { SSB } & 0 \\ \text { LB } & 0.30 \mathrm{~Hz} \\ \text { GB } & 0 \\ \text { PC } & 1.00 \\ & \end{array}$ 


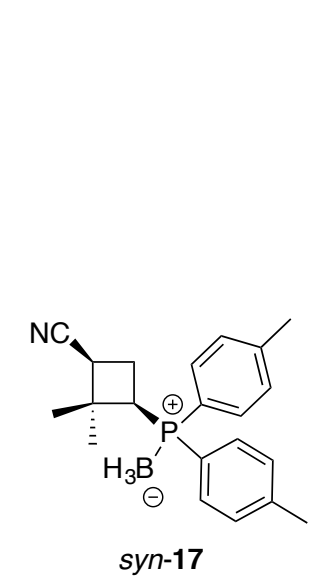

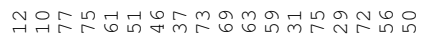

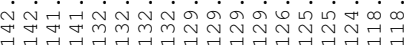

$\checkmark 1$

$\left|V^{m}\right|^{m} V^{n+n}$

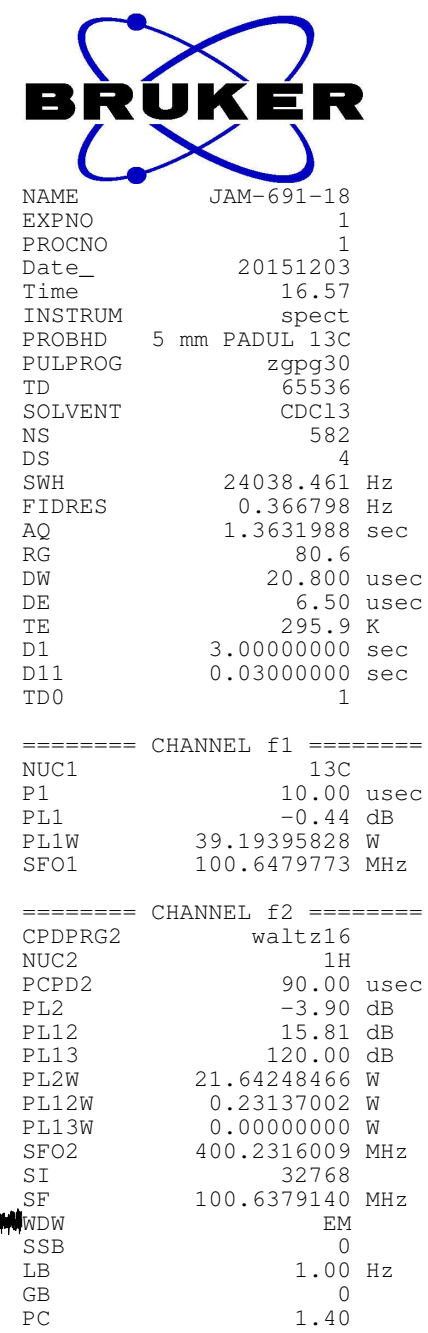



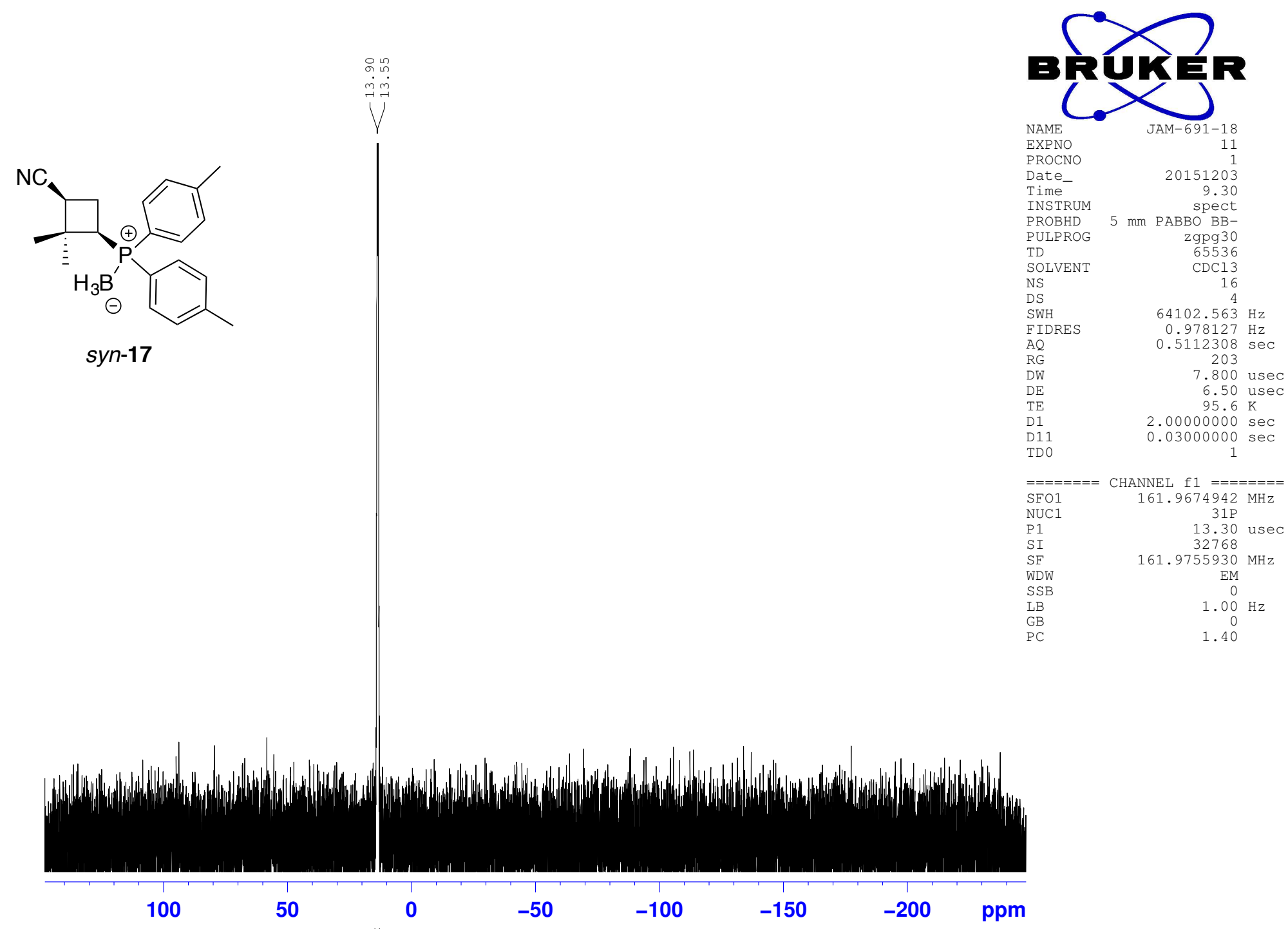


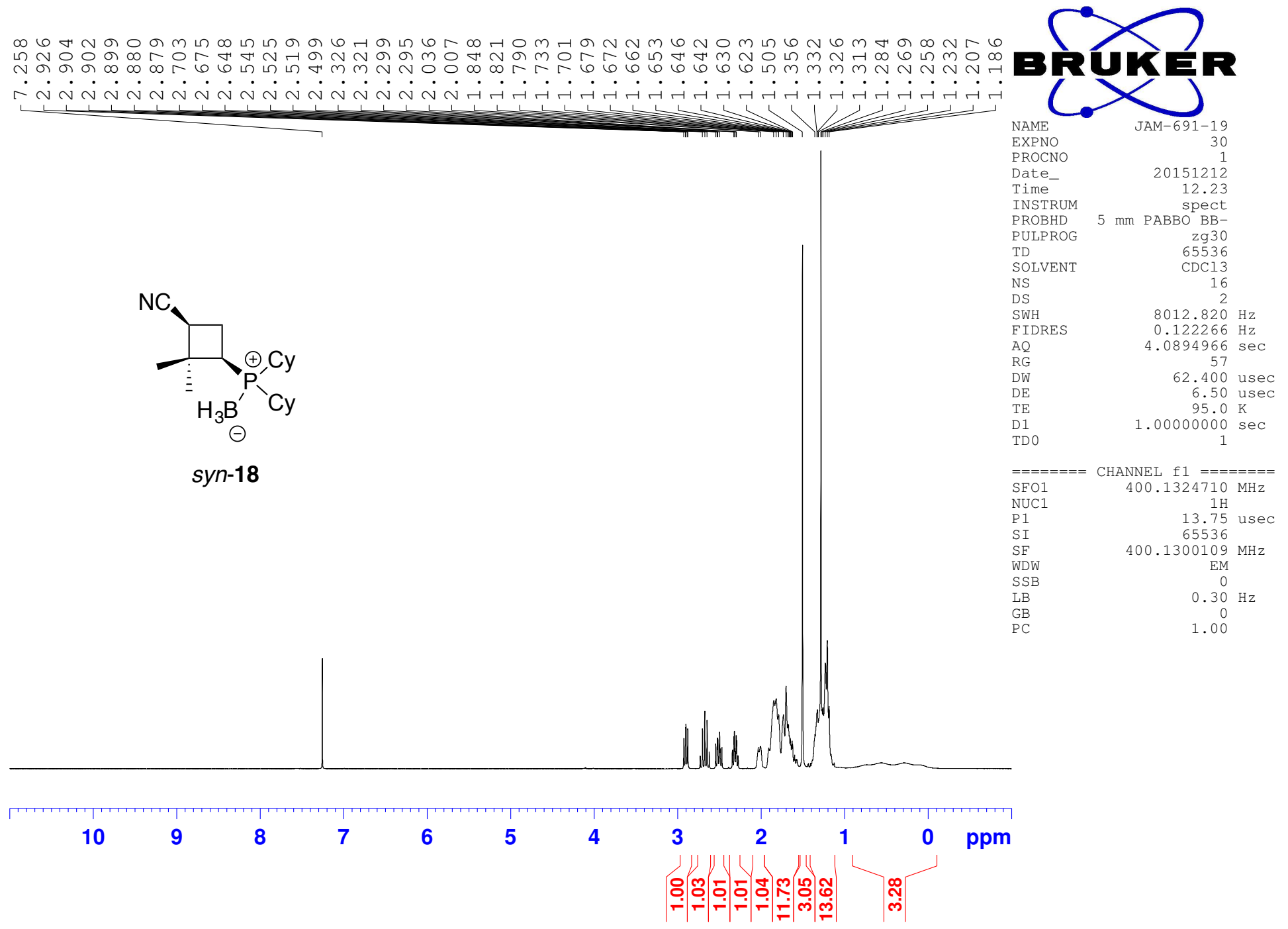




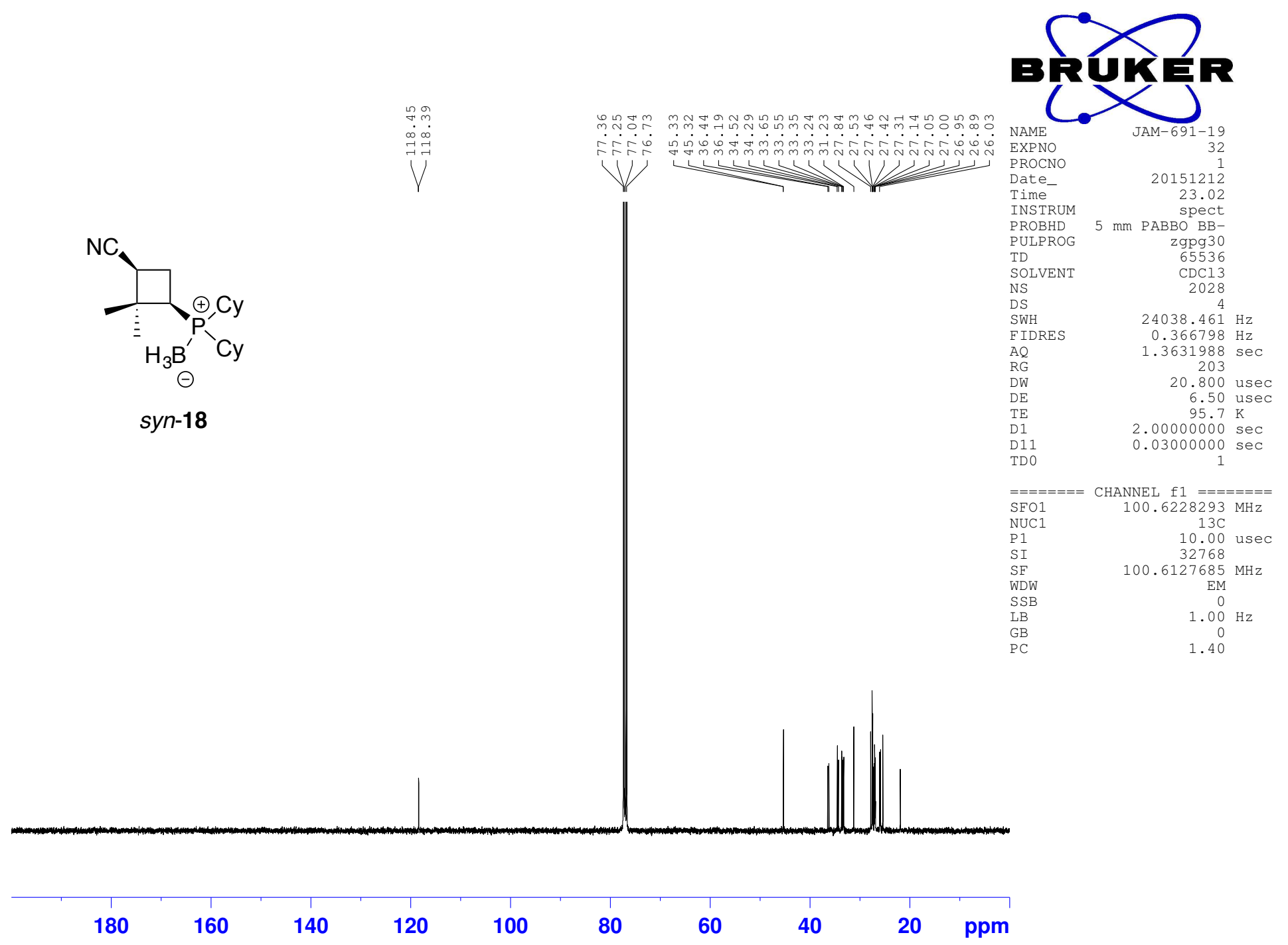




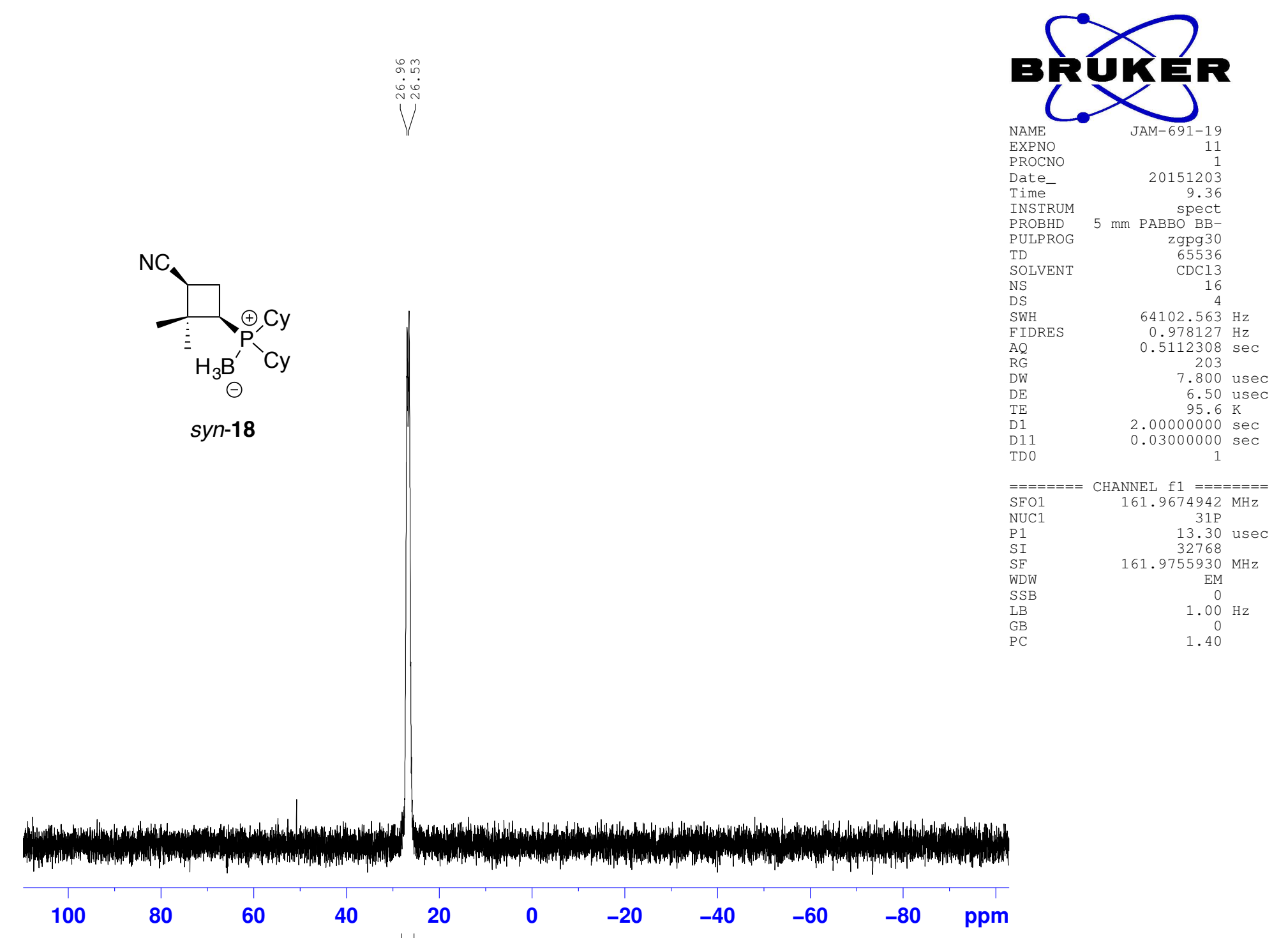




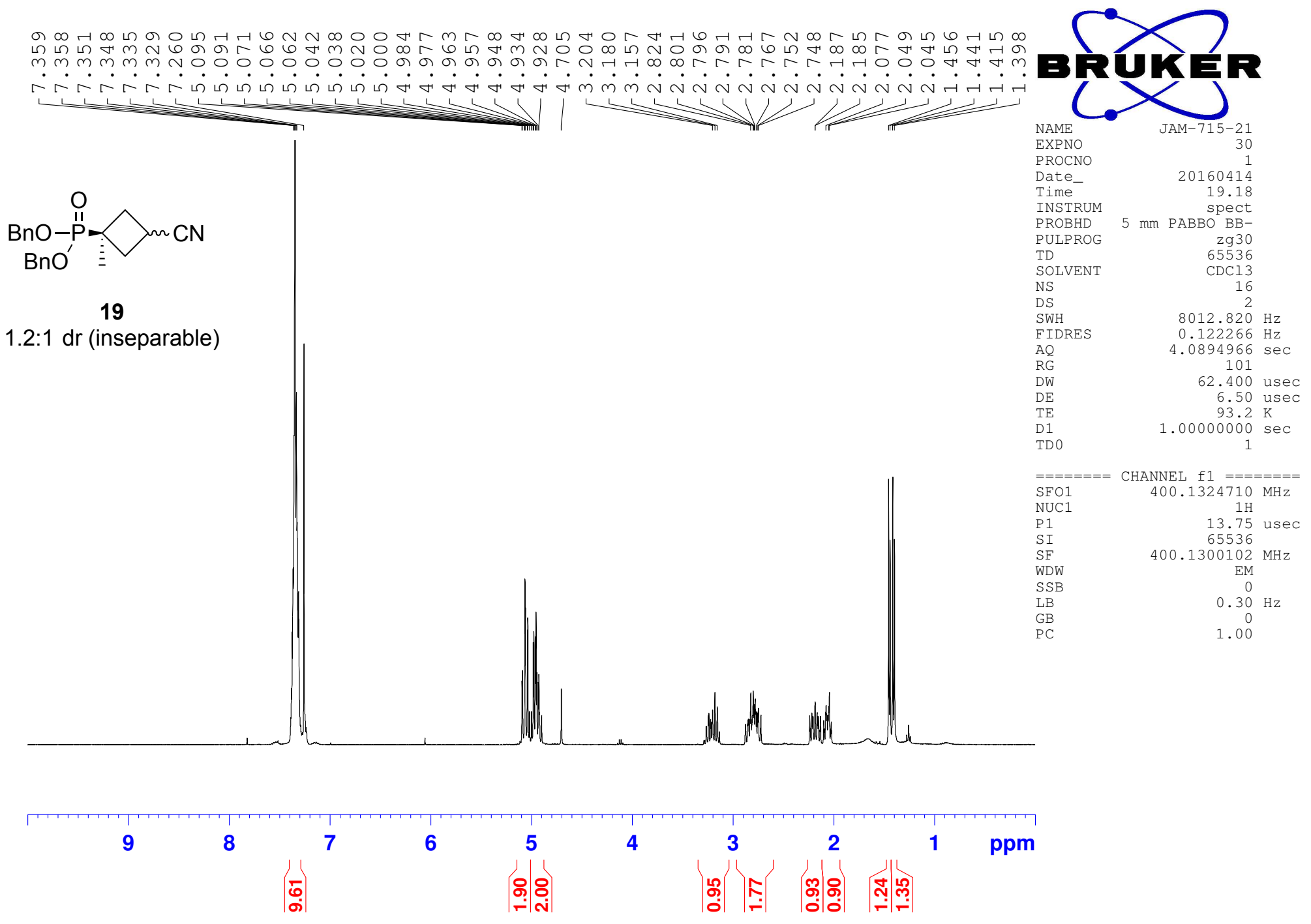



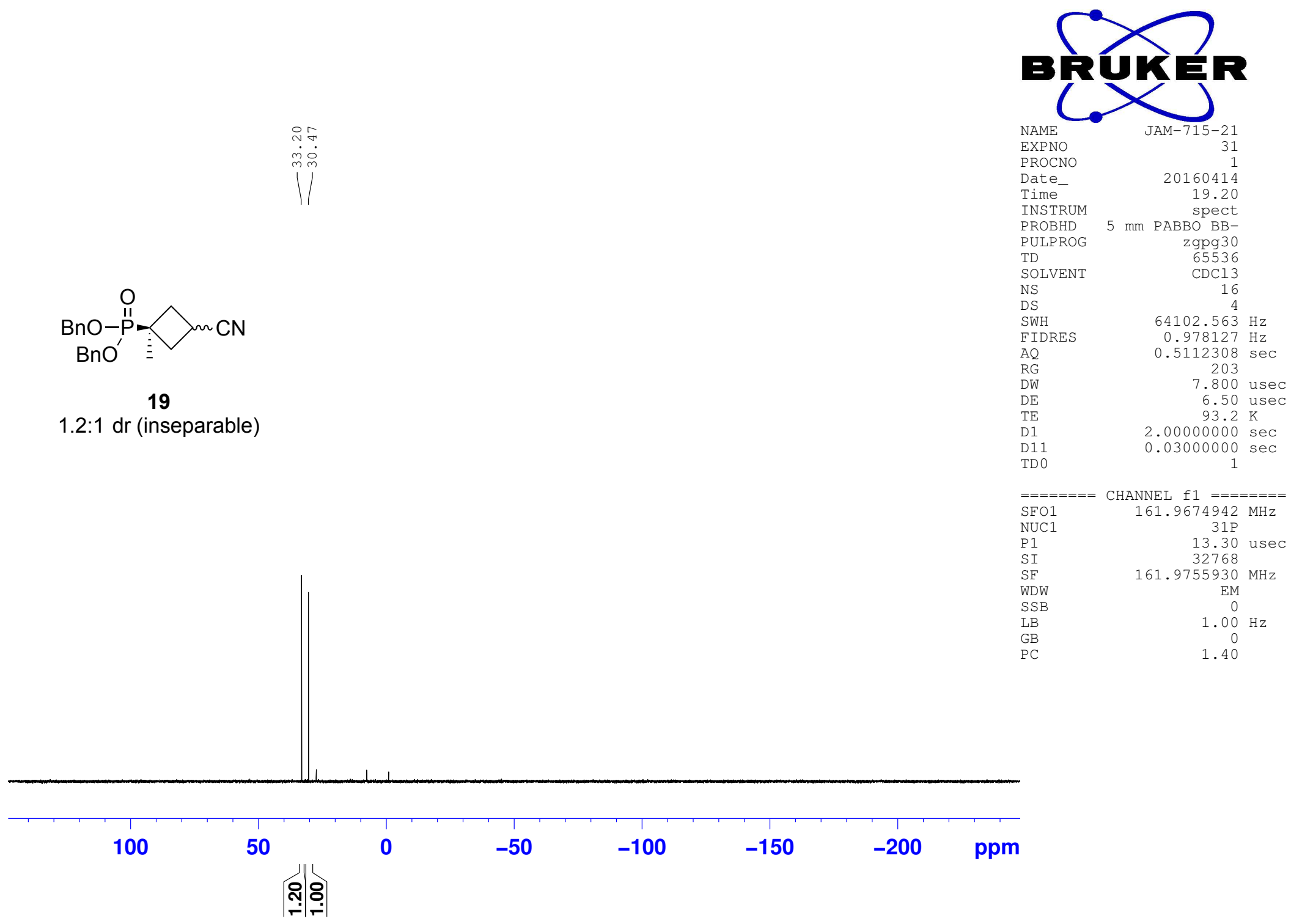


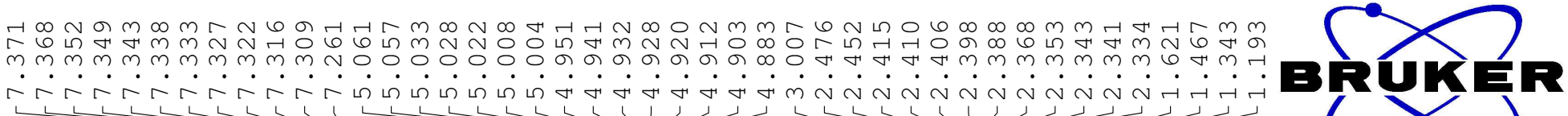

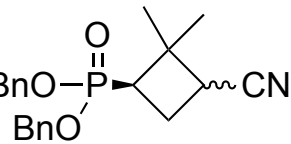

20

1.1:1 dr (inseparable)

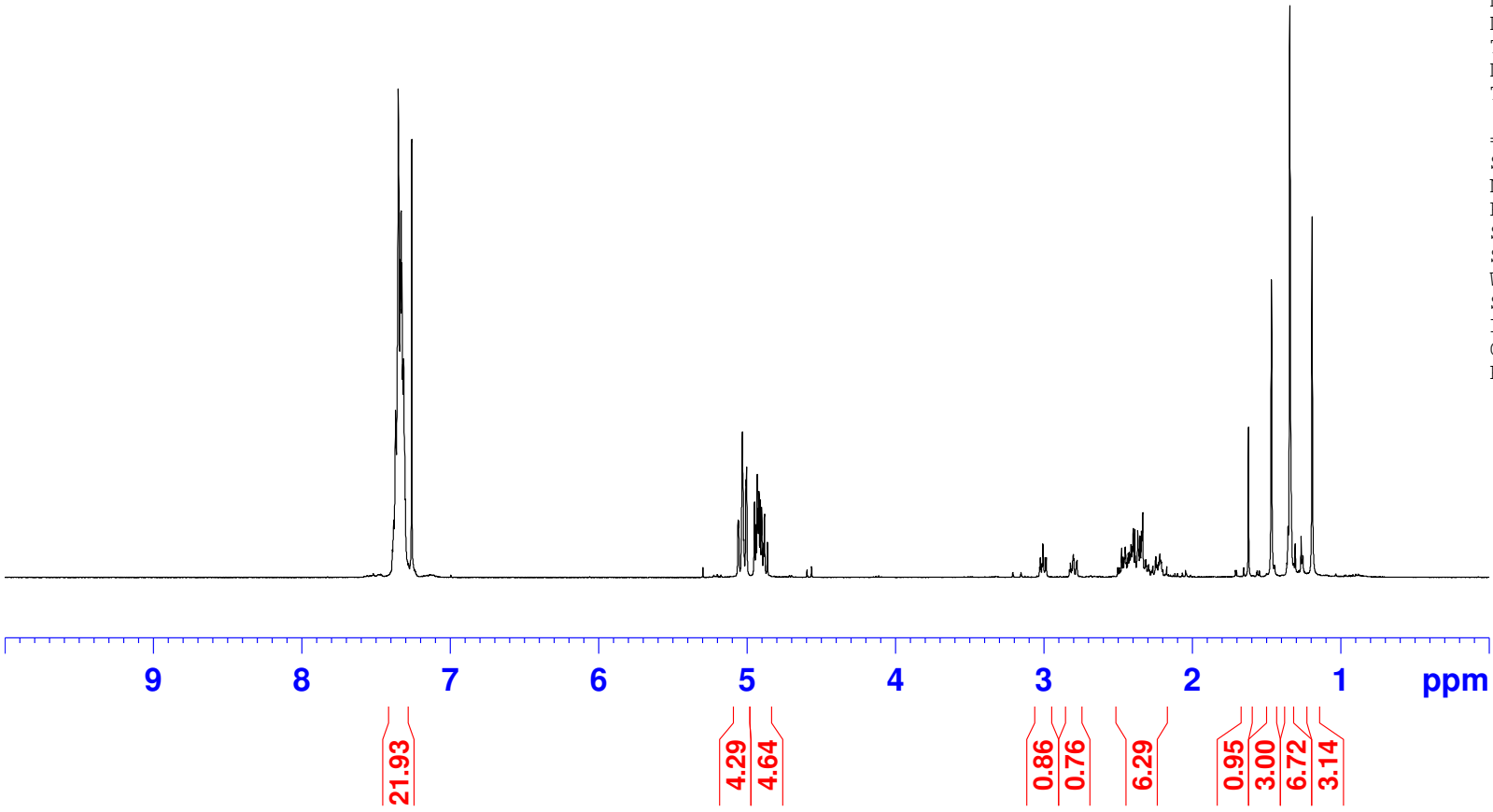

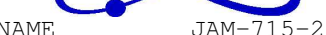

XXNO

INSTRU

spect
ROBHD
PULPROG

20160420

160420
17.25

TD

SOLVEN

DS

DS

FIDRES

RG

RG
$D W$
$D E$

D 1

zg30
65536

16

$8012.820 \mathrm{~Hz}$ $4.0894966 \mathrm{sec}$

62.400 usec

6.50 usec

$1.00000000 \mathrm{sec}$

fHANEL f1 $========$

$400.1324710 \mathrm{MHz}$
$1 \mathrm{H}$

$\mathrm{SI}$
$\mathrm{SF}$

WDW
$400.1300092 \mathrm{MH}$

EM

0
$0.30 \mathrm{~Hz}$

1.00 

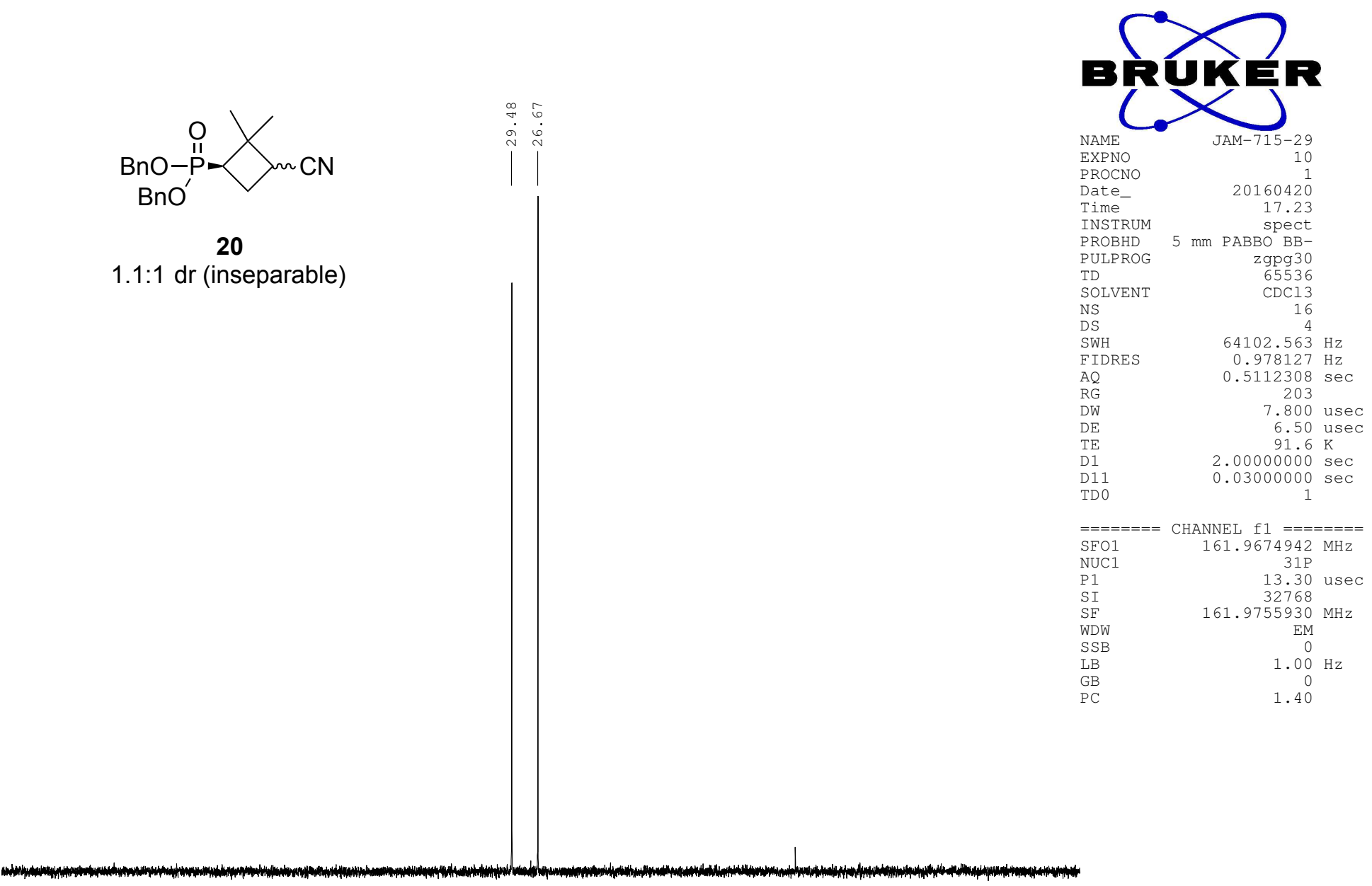

1.1:1 dr (inseparable)

(0) 

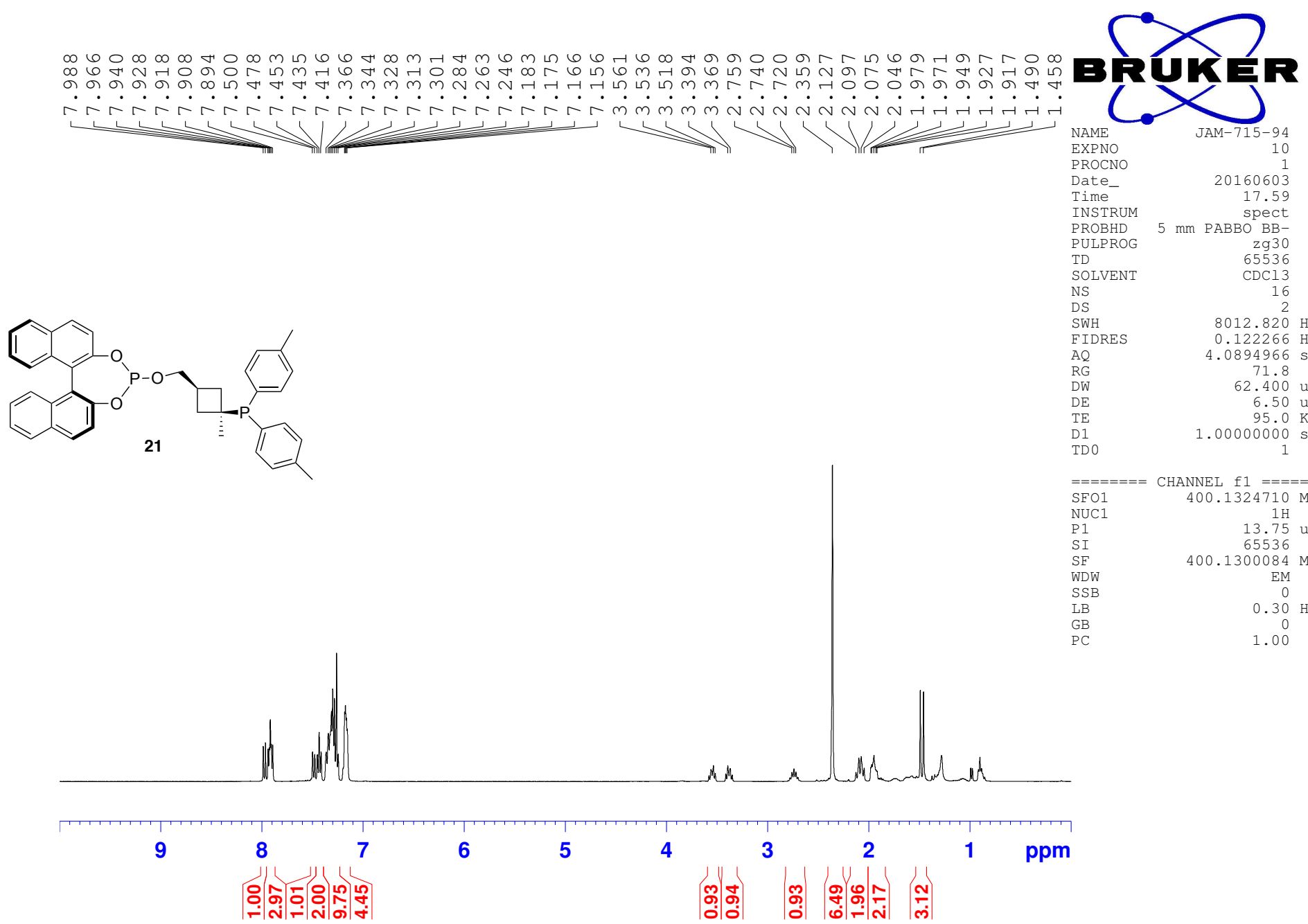

NAME
EXPNO
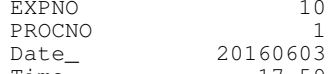

$\begin{array}{lr}\text { Time } & 17.59\end{array}$

spect
INSTRUM PROBHD

$\begin{array}{lr}\text { PULPROG } & \text { zg30 } \\ \text { TD } & 65536 \\ \text { SOLVENT } & \text { CDC13 }\end{array}$

DS

SWH

$\mathrm{AQ}$
$\mathrm{RG}$

RG
DW

DW
DE
TE
D1

$8012.820 \mathrm{~Hz}$ $4.0894966 \mathrm{sec}$

62.400 usec

6.50 use $1.00000000 \mathrm{sec}$

$=======$ CHANNEL $f 1 \quad=======$
SFO1
$400.1324710 \mathrm{MHz}$

$\begin{array}{lr}\text { SFO1 } & 400.1324710 \mathrm{MHz} \\ \text { NUC1 } & 1 \mathrm{H}\end{array}$

$\begin{array}{lr}\text { NUC1 } 1 & 1 \mathrm{H} \\ \mathrm{P} 1 & 13.75 \text { usec }\end{array}$

65536
SF

WDW

SSB
LB
GB

0
$0.30 \mathrm{~Hz}$
0

1.00

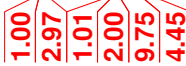

| 

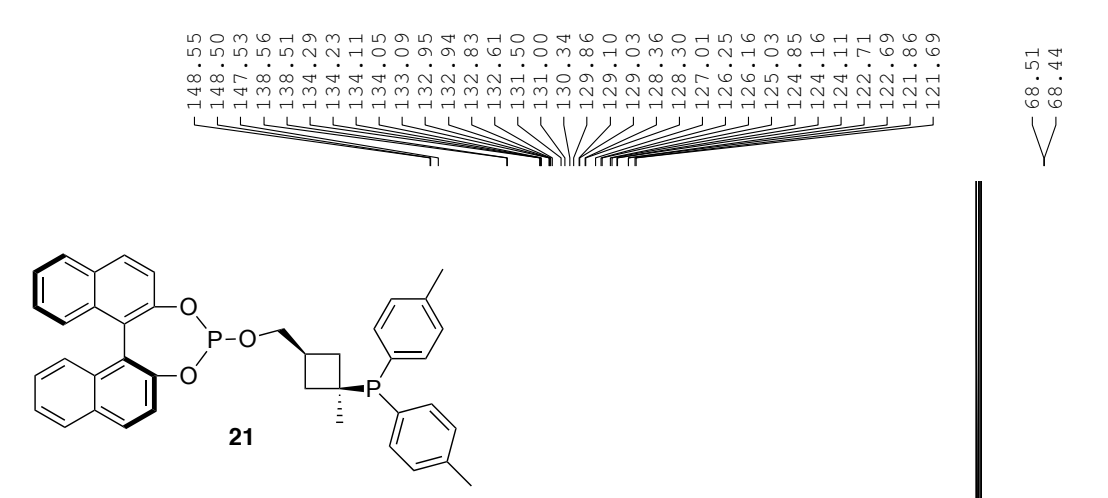

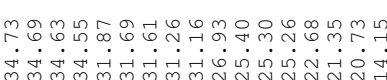
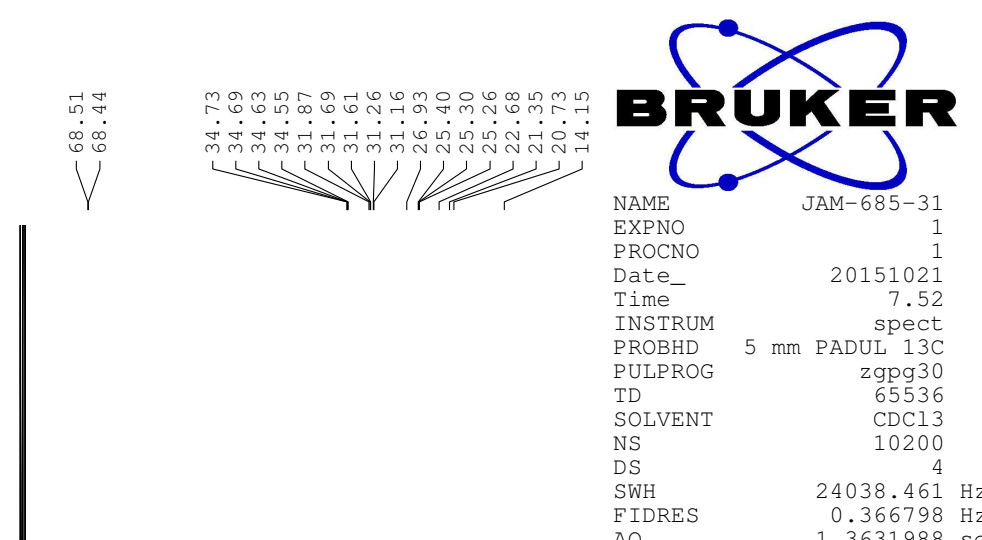

NAME
EXPNO

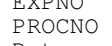

Date

$\begin{array}{lr}\text { Time } & 20151021 \\ \text { INSTRUM } & \text { spect }\end{array}$

PROBHD $5 \mathrm{~mm}$ PADUL $13 \mathrm{C}$
PULPROG

$\begin{array}{lr}\text { TD } & 65536 \\ \text { SOLVENT } & \text { CDC13 }\end{array}$

$\begin{array}{lr}\text { NS } & 10200 \\ \text { DS } & 4\end{array}$

$\begin{array}{ll}\text { SWH } & 24038.461 \mathrm{~Hz} \\ \text { FIDRES } & 0.366798 \mathrm{~Hz}\end{array}$

$\begin{array}{lr}\text { FIDRES } & 0.366798 \mathrm{~Hz} \\ \text { AQ } & 1.3631988 \mathrm{sec}\end{array}$

$\begin{array}{lr}\text { RG } & 50.8 \\ \text { DW } & 20.800 \text { usec }\end{array}$

$\begin{array}{lr}\text { DE } & 6.50 \text { usec } \\ \text { TE } & 296.3 \mathrm{~K}\end{array}$

$\begin{array}{lr}\text { TE } & 296.3 \mathrm{~K} \\ \text { D1 } & 3.00000000 \mathrm{sec}\end{array}$

D11 $0.03000000 \mathrm{sec}$

$========$ CHANNEL f $1=======$
$13 \mathrm{C}$

$\begin{array}{lr}\mathrm{NUC1} & 13 \mathrm{C} \\ \mathrm{P} 1 & 10.00 \text { usec }\end{array}$

$\begin{array}{ll}\text { PL1 } & -0.44 \mathrm{~dB} \\ \text { PL1W } & 39.19395828\end{array}$

$\begin{array}{ll}\text { PL1W } & 39.19395828 \mathrm{~W} \\ \text { SFO1 } & 100.6479773 \mathrm{MHz}\end{array}$

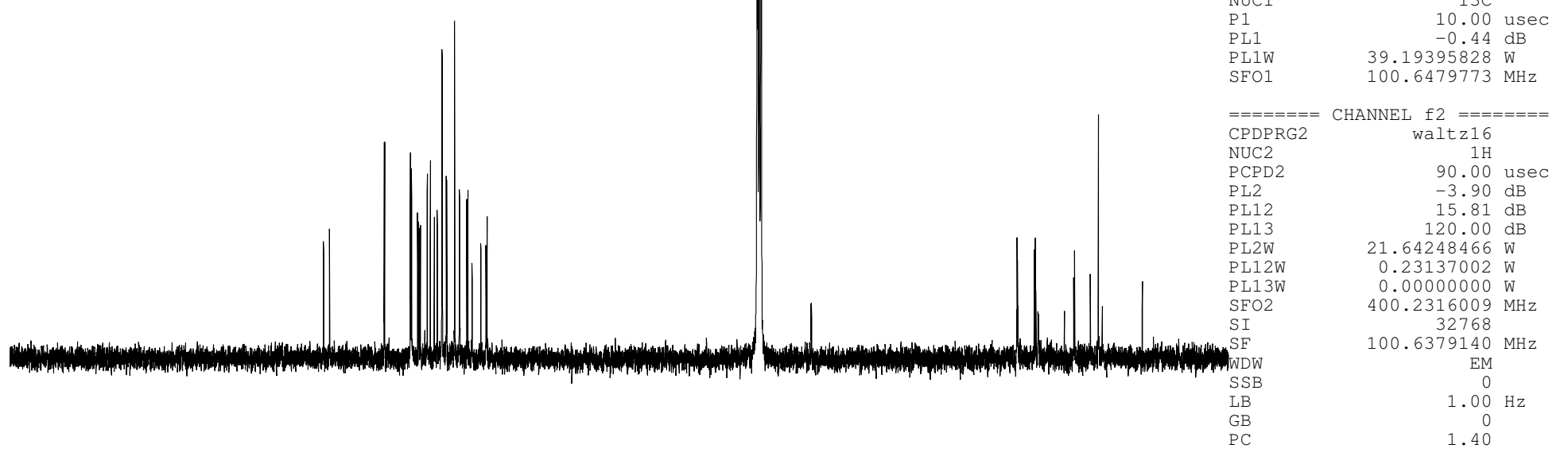




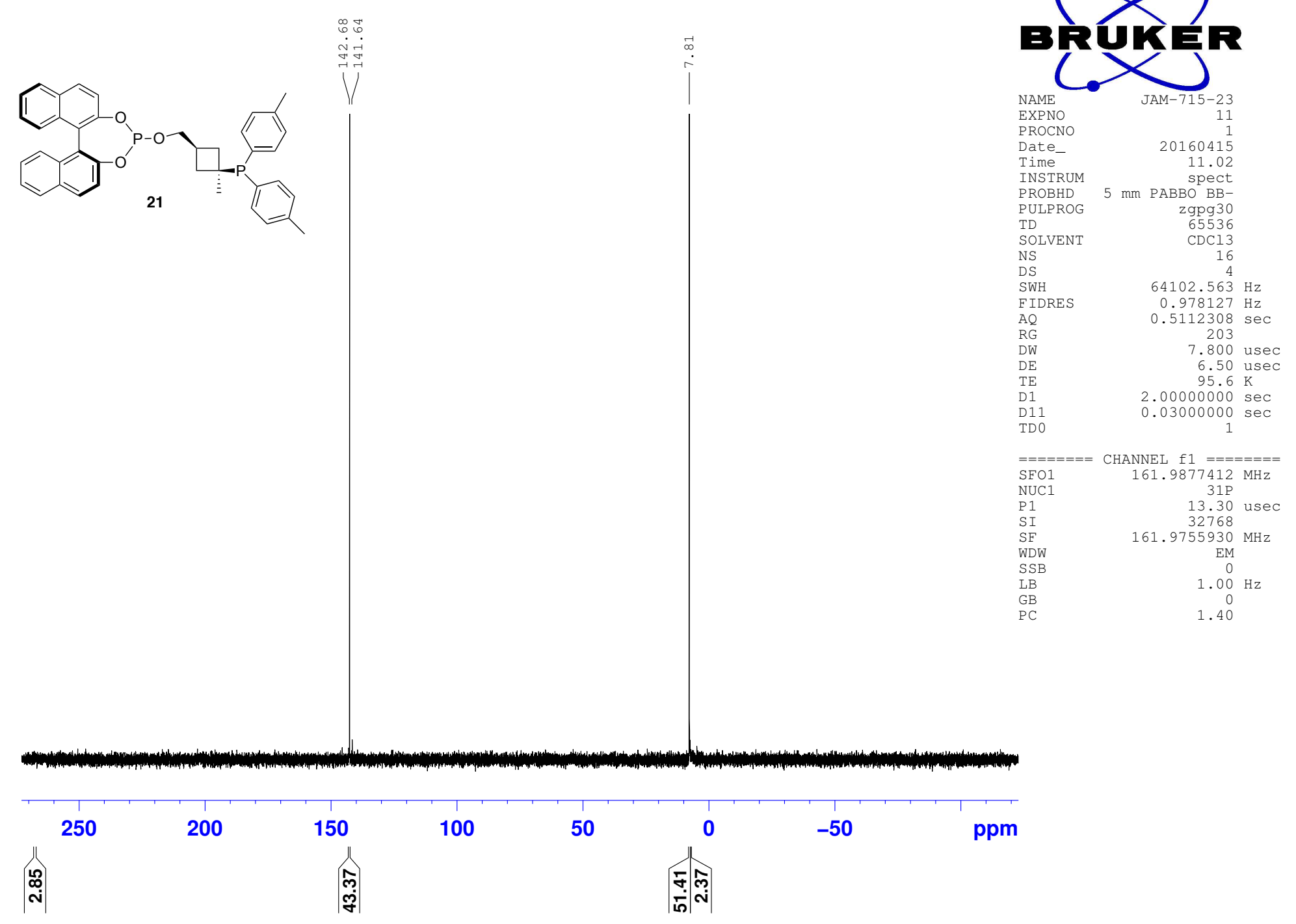




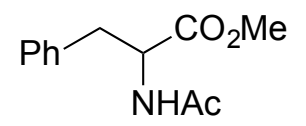

$(+/-)$

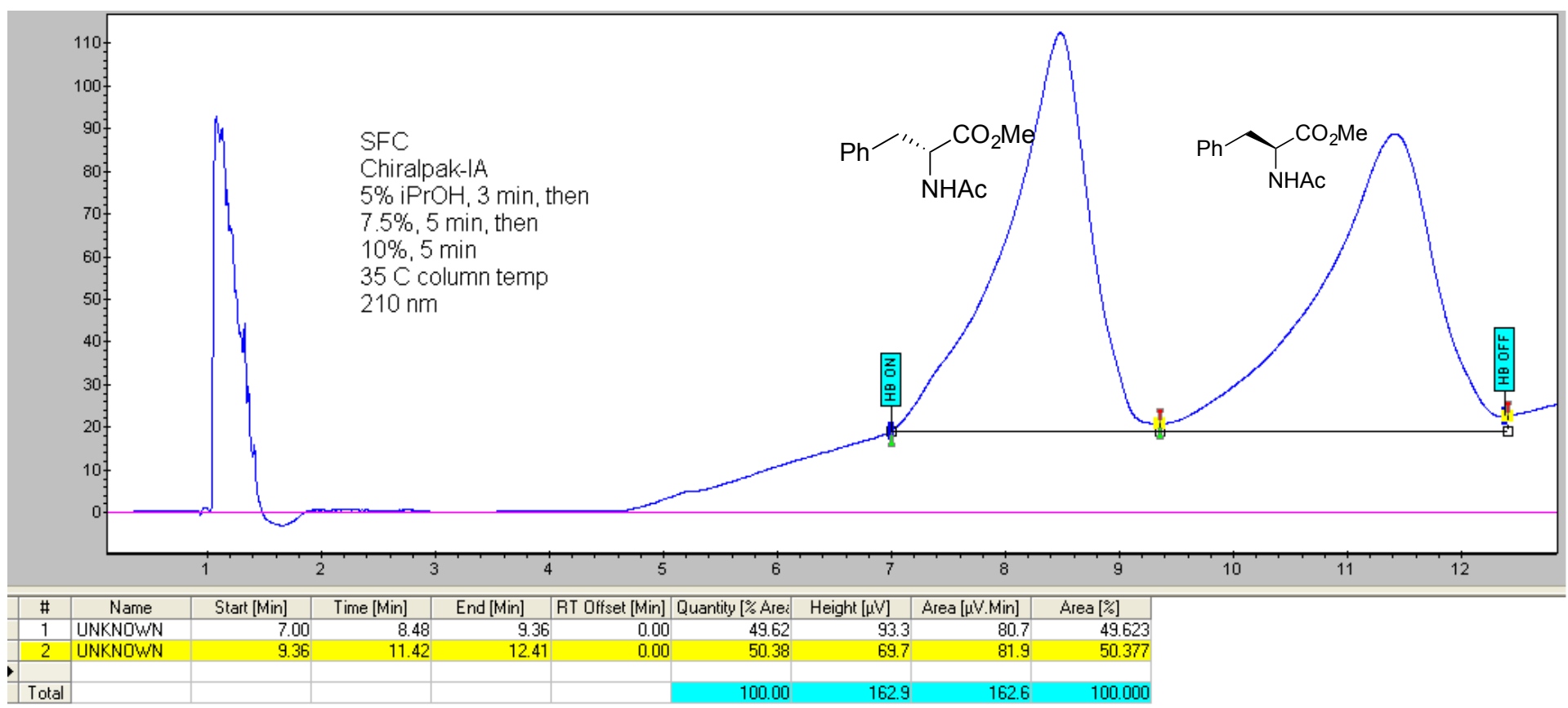




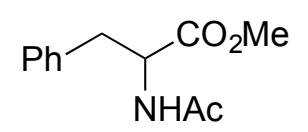

23

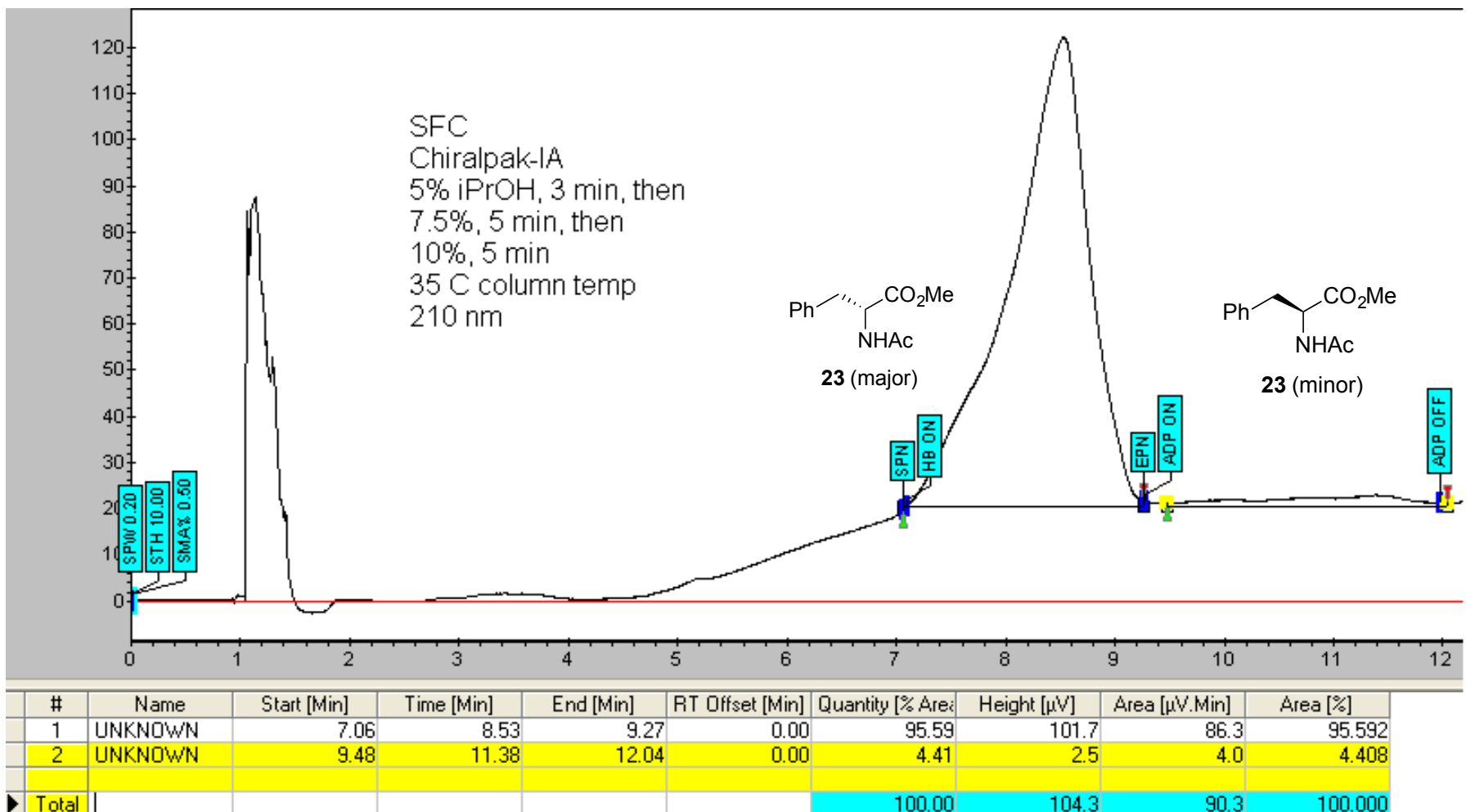

| Total |

100.00

104.3

$90.3 \quad 100.000$ 